DISTRIBUTION SHEET

\begin{tabular}{|c|c|c|c|c|c|}
\hline \multirow{2}{*}{$\begin{array}{l}\text { To } \\
\text { Distribution }\end{array}$} & \multirow{2}{*}{\multicolumn{3}{|c|}{$\begin{array}{l}\text { From } \\
\text { Characterization Support }\end{array}$}} & \multicolumn{2}{|c|}{ Page 1 of 4} \\
\hline & & & & \multicolumn{2}{|c|}{ Date $9-30-94$} \\
\hline \multirow{2}{*}{\multicolumn{4}{|c|}{$\begin{array}{l}\text { Project Title/Work Order } \\
\text { Tank Characterization Reference Guide }\end{array}$}} & EDT No. & 159099 \\
\hline & & & & ECN No & NA \\
\hline Name & MSIN & $\begin{array}{c}\text { Text } \\
\text { With } \\
\text { All } \\
\text { Attach. }\end{array}$ & $\begin{array}{l}\text { Text } \\
\text { Only }\end{array}$ & $\begin{array}{l}\text { Attach./ } \\
\text { Appendix } \\
\text { Only }\end{array}$ & $\begin{array}{l}\text { EDT/ECN } \\
\text { Only }\end{array}$ \\
\hline
\end{tabular}

OFFSITE

Sandia National Laboratory

P.0. Box 5800

MS-0744, Dept. 6404

Albuquerque, NM 87815

D. Powers

$x$

Nuclear Consulting Services Inc.

P.0. Box 29151

Columbus, $\mathrm{OH}$ 43229-01051

J. L. Kovach

$x$

Chemical Reaction Sub-TAP

202 Northridge Court

Lindsborg, KS 67456

B. C. Hudson

$x$

Tank Characterization Panel

Senior Technical Consultant

Contech

6301 Indian School Road NE, Suite 614

Albuquerque, NM 87110

J. Arvizu

$x$

U.S. Department of Energy - Headquarters

Office of Environmental Restoration and Waste Management EM-563

12800 Middlebrook Road

Germantown, MD 20874

K. T. Lang

J. A. Poppitti

$x$
$x$

$\underline{S A I C}$

2030 Centry Boulevard

Suite 200-B

Germantown, MD 20874 


\section{DISCLAIMER}

Portions of this document may be illegible in electronic image products. Images are produced from the best available original document. 
Los Alamos Laboratory

CST-14 MS-J586

P.0. Box 1663

Los Alamos, NM 87545

S. F. Agnew (6)

Los Alamos Technical Associates

750 Swift Blvd., Suite 4

Richland, WA 99352

A. T. DiCenso

$X$

Ogden Environmental

101 East Wellsian Way

Richland, WA 99352

R. J. Anema

$x$

CH2M Hil1

P.0. Box 91500

Bellevue, WA 98009-2050

M. McAfee

$x$

Tank Advisory Panel

102 Windham Road

Oak Ridge, TN 37830

D. 0. Campbe11

$x$

ONSITE

Department of Ecology
A. B. Stone (4)
N1-05
X

Department of Energy - Richland Operations

J. M. Clark

R. E. Gerton

ICF-Kaiser Hanford Company

C. H. Brevick

L. A. Gadd is

R. B. Johnson

W. W. Pickett
S7-54 X

S7-54 $X$

G7-56 $X$

G7-57 $\quad X$

G7-56 $X$

G7-57 $\quad X$

Pacific Northwest Laboratories
W. J. Apley
R. M. Bean
$57-71$
P8-08
S. F. Bobrowski
K7-28
$x$
$x$
$\mathrm{X}$ 
P. G. Eller

E. J. Eschbach

S. A. Hartley

J. G. Hill

L. K. Holton

B. M. Johnson

M. E. Lerchen

G. J. Lumetta

B. D. McVeety

I. C. McVeety

P. J. Mellinger

A. F. Noonan

L. R. Pederson

K. M. Remund

$\begin{array}{ll}\text { B1-40 } & \cdot \\ K 7-15 & X \\ K 5-12 & X \\ K 7-97 & X \\ \text { P7-43 } & X \\ K 1-78 & X \\ K 7-94 & X \\ P 7-25 & X \\ K 6-63 & X \\ K 7-22 & X \\ \text { P7-22 } & X \\ \text { B1-40 } & X \\ K 2-44 & X \\ K 5-12 & X \\ & X\end{array}$

Westinghouse Hanford Company

H. Babad

D. A. Barnes

A. L. Boldt

G. L. Borsheim

D. R. Bratzel

T. M. Brown

T. H. Bushaw

M. P. Campbe11

R. J. Cash

G. M. Christensen

W. L. Cowley

M. L. Deffenbaugh

C. DeFigh-Price

R. A. Dodd

G. L. Dunford

S. J. Eberlein

D. B. Engelman

K. 0. Fein

J. S. Garfield

K. D. Gibson

C. E. Golberg

J. M. Grigsby

R. D. Gustavson

C. S. Haller

H. W. Heacock

D. L. Herting

B. A. Higley

G. Jansen

G. D. Johnson

K. K. Kawabata

N. W. Kirch

M. J. Kupfer

G. A. Meyer

W. C. Miller

W. C. Mills

C. T. Narquis

R. H. Palmer

M. A. Payne

S. H. Rifaey
S7-30

Rl-51

H5-49

H5-27

S7-31

R2-12

T6-30

R2-86

S7-15

H4-21

H4-61

R2-06

S7-30

R2-70

R2-50

S7-31

R1-49

H4-63

H5-49

H4-6I

H5- 49

H4-62

R1-51

R2-12

S7-81

T6-09

H5-27

H6-33

S7-15

T6-50

R2-11

H5-49

S4-54

S4-55

S4-58

T6-50

R2-58

S7-14

S2-45

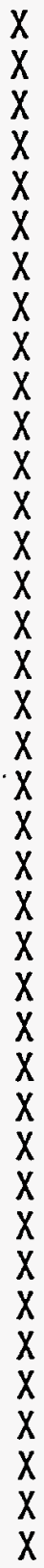




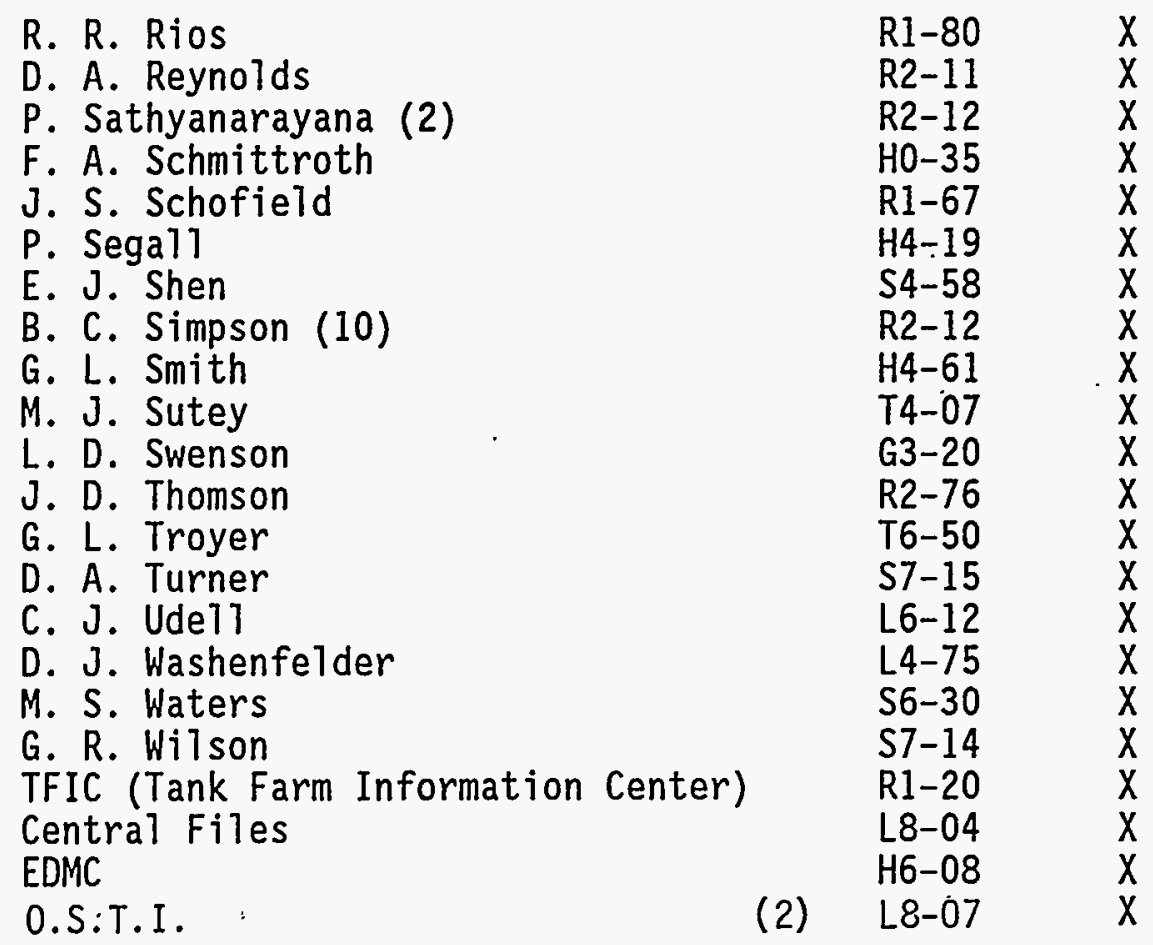




\section{4 \\ SEP 301994 ENGINEERING DATA TRANSMITTAL}

2. To: (Receiving Organization) Characterization Support

5. Proj./Prog./Dept./Div.: WM/Characterization

8. Originator Remarks:

The document is an introduction to the Hanford Site and provides initial technical and contextual information for understanding the waste tanks and characterization effort.
3. From: (Originating Organization) Characterization Support

6. Cog. Engr.:

B. C. Simpson

4. Related EDT No.:

7. Purchase Order No.:

NA

9. Equip./Component No.:

NA

10. System/Bldg./Facility:

Tank Farms

11. Receiver Remarks:

12. Major Assm. Dwg. No.:

NA

13. Permit/Permit Application No.: NA

14. Required Response Date: Sept. 30, 1994

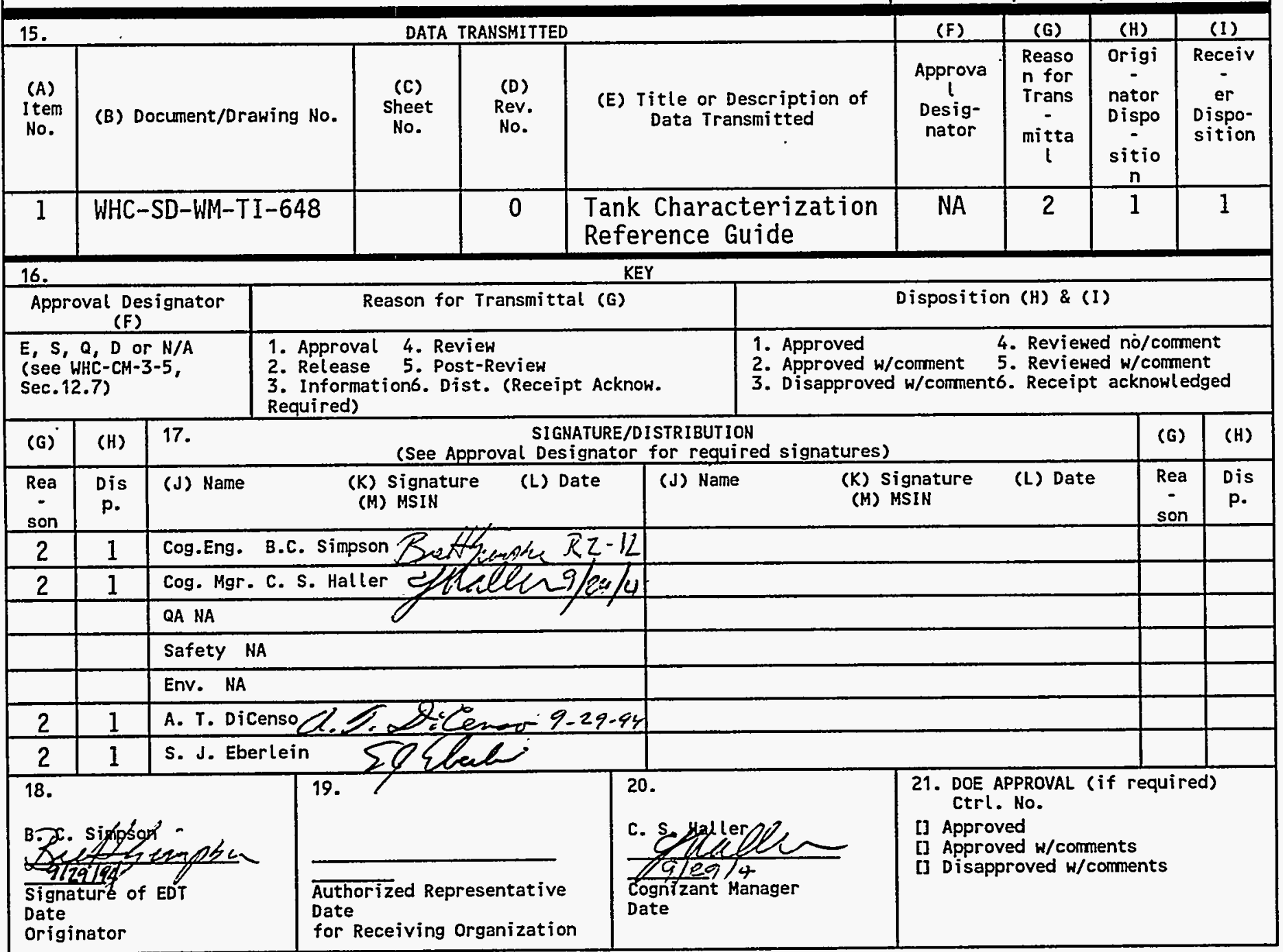

BD-7400-172-2 (04/94) GEF097 


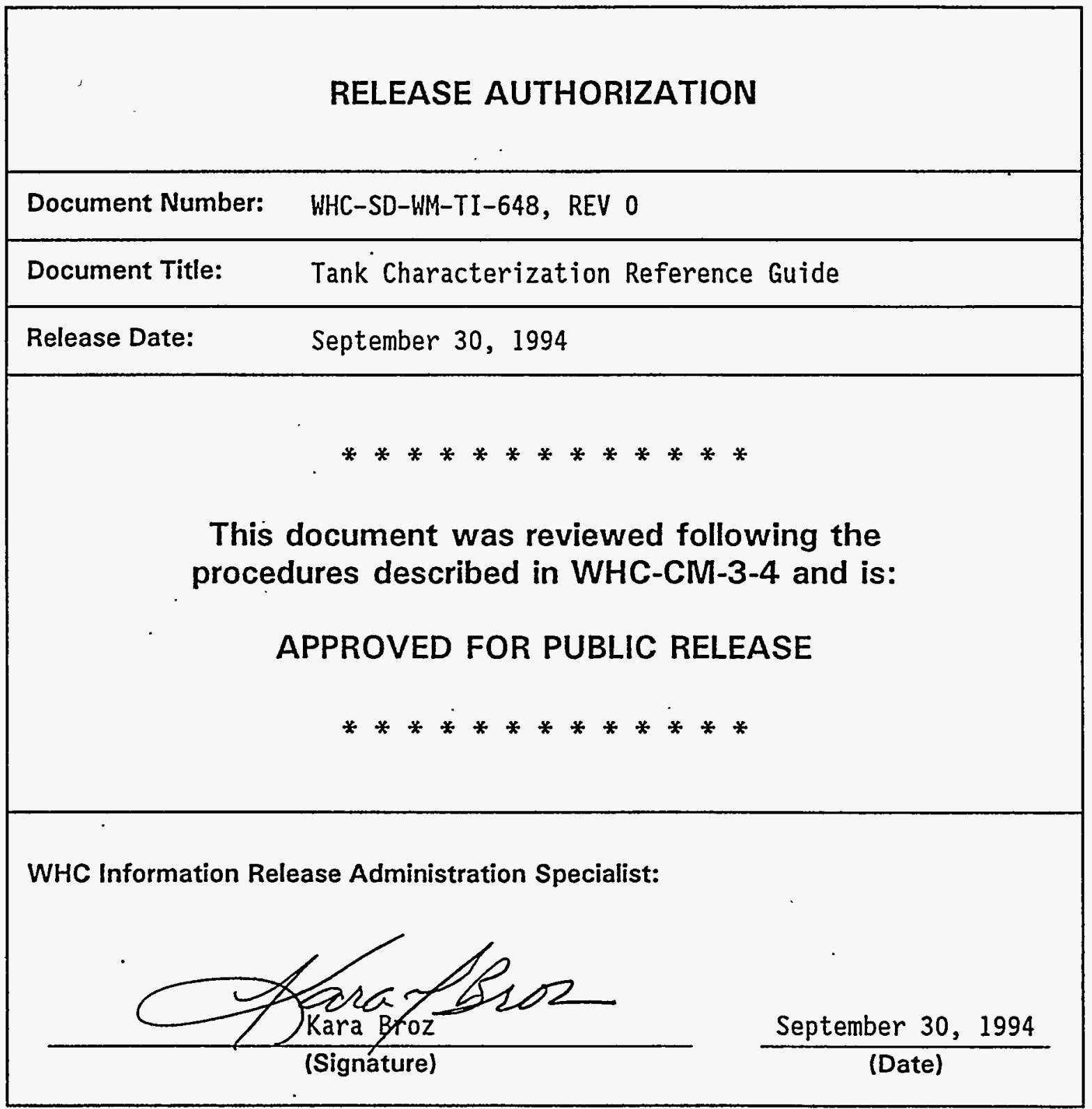




\section{Title}

3. Number

4. Rev No.

Tank Characterization Reference Guide

WHC-SD-HM-TI -648

0

5. Key Hords

Waste Characterization; Single-Shell Tank; DoubleShel1 Tank; Tank Characterization Report; Hanford Site History; Introduction to Tank Farms; Reference for Tank Farms

\section{APPROVED FOR PUBLIC RELEASE}

6. Author

Name: B. C. Simpson

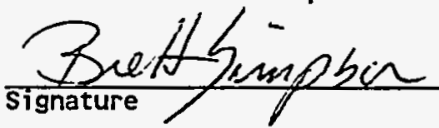

Name: A. T. DiCenso

signature

Organization/Charge code 7E720/N4D2F

\section{Abstract}

This document provides an introduction to those unfamiliar with the Hanford Site, the tank farms, and the associated history surrounding the generation, storage, and management of the waste streams from the various production and separation processes at Hanford.

8. PURPOSE AND USE OF DOCUMENT - This document was prepared for use withim the U.S. Department of Energy and its contractors it is to be used oly to perform, direct, or integrate work under U.S. Department of Energy contracts. This document is not approved for public release unsil reviewed.

PATENT STATUS - This document copty, since it is transmitted in advance of patent clearance, is made available in confidence solely for use in performapee of work undec contracts with the U.S. Department of Energy. This document is not te be published nor its contents gtherwise disseminated or used for purposes other than specified above before patent approval for such release on use has been oecured, upon request, from the Patent Counsel, U.S. Departmont onergy Field Office, Richland, WA.

DISCLAIMER - This report was prepared as an account of work sponsored by an agency of the United States Government. Neither the United States Government nor any agency thereof, nor any of their employees, nor any of their contractors, subcontractors or their employees, makes any warranty, express or implied, or assumes any legal liability or responsibility for the accuracy, completeness, or any third party's use or the results of such use of any information, apparatus, product, or process disclosed, or represents that its use would not infringe privately owned rights. Reference herein to any specific commercial product, process, or service by trade name, trademark, manufacturer, or otherwise, does not necessarily constitute or imply its endorsement, recommendation, or favoring by the United States Government or any agency thereof or its contractors or subcontractors. The views and opinions of authors expressed herein do not necessarily state or reflect those of the United States Government or any agency thereof.

10. RELEASE STAMP

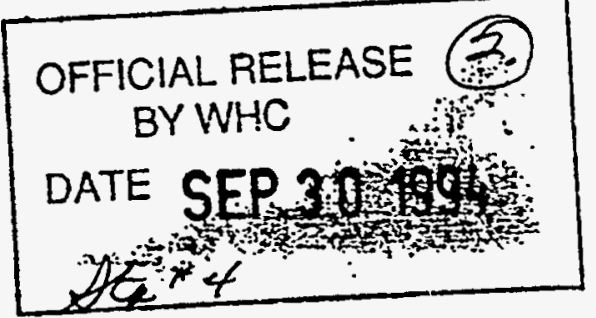

9. Impact Level NA 


\title{
Tank Characterization Reference Guide
}

\author{
D.S. De Lorenzo
}

A.T. DiCenso

D.B. Hiller

K.W. Johnson

J.H. Rutherford

D.J. Smith

Los Alamos Technical Associates, Incorporated

\section{B.C. Simpson \\ Westinghouse Hanford Company}

\section{DISCLAIMER}

This report was prepared as an account of work sponsored by an agency of the United States Government. Neither the United States Government nor any agency thereof, nor any of their employees, makes any warranty, express or implied, or assumes any legal liability or responsibility for the accuracy, completeness, or usefulness of any information, apparatus, product, or

Date Published

September 1994 process disclosed, or represents that its use would not infringe privately owned rights. Reference herein to any specific commercial product, process, or service by trade name, trademark, manufacturer, or otherwise does not necessarily constitute or imply its endorsement, recommendation, or favoring by the United States Government or any agency thereof. The views and opinions of authors expressed herein do not necessarily state or reflect those of the United States Government or any agency thereof.

\footnotetext{
Prepared for Westinghouse Hanford Company by

Los Alamos Technical Associates

8633 Gage Blvd.

Kennewick, WA 99336
}

LATA-TCR-9491, Rev. 0

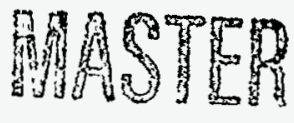




\section{EXECUTIVE SUMMARY}

Characterization of the Hanford Site high-level waste storage tanks supports safety issue resolution; operations and maintenance requirements; and retrieval, pretreatment, vitrification, and disposal technology development. Technical, historical, and programmatic information about the waste tanks is often scattered among many sources, if it is documented at all. This Tank Characterization Reference Guide, therefore, serves as a common location for much of the generic tank information that is otherwise contained in many documents. The report is intended to be an introduction to the issues and history surrounding the generation, storage, and management of the liquid process wastes, and a presentation of the sampling, analysis, and modeling activities that support the current waste characterization. This report should provide a basis upon which those unfamiliar with the Hanford Site tank farms can start their research. 


\section{CONTENTS}

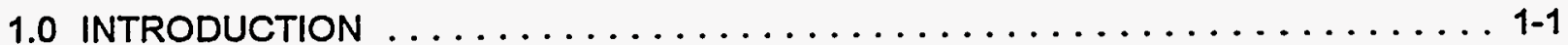

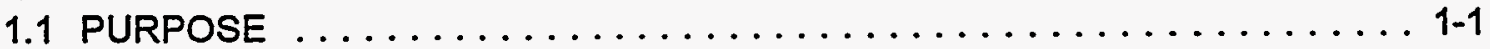

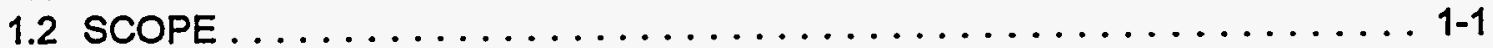

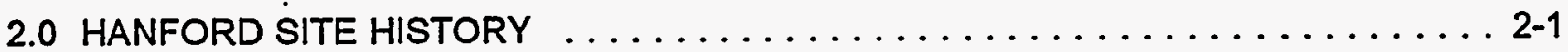

2.1 PRODUCTION AND FACILITIES HISTORY $\ldots \ldots \ldots \ldots \ldots \ldots \ldots \ldots \ldots \ldots . \ldots \ldots$

2.2 HANFORD SITE TANK FARMS $\ldots \ldots \ldots \ldots \ldots \ldots \ldots \ldots \ldots \ldots \ldots$

3.0 WASTE TYPES AND MANAGEMENT PRACTICES . . . . . . . . . . . . 3-1

3.1 WASTE DESCRIPTION AND GENERATION $\ldots \ldots \ldots \ldots \ldots \ldots \ldots \ldots \ldots . \ldots \ldots$

3.1.1 Bismuth Phosphate Process . . . . . . . . . . . . . . . 3-4

3.1.2 Tributyl Phosphate (TBP) Uranium Recovery Process . . . . . . . . . 3-4

3.1.3 Reduction Oxidation (REDOX) Process . . . . . . . . . . . . . 3-4

3.1.4 Plutonium-Uranium Extraction (PUREX) Process . . . . . . . . . . 3-6

3.1 .5 Other Processes . . . . . . . . . . . . . . . . . . . 3-8

3.1.6 Current Waste Sources ... . . . . . . . . . . . 3-10

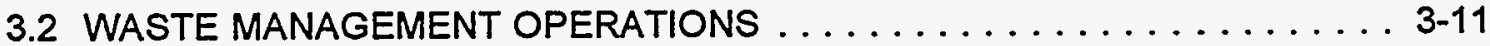

3.2.1 Tank Waste Remediation System . . . . . . . . . . . 3-11

3.2.2 Evaporation and Concentration . . . . . . . . . . . . 3-12

3.2.3 Ferrocyanide Scavenging ................ 3-14

3.2.4 Hanford Grout Disposal Program . . . . . . . . . . . . . 3-15

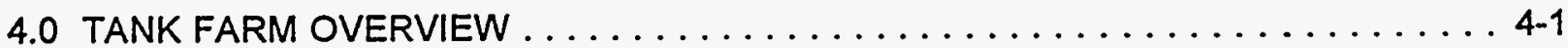

4.1 SINGLE-SHELL TANK BACKGROUND $\ldots \ldots \ldots \ldots \ldots \ldots \ldots \ldots \ldots \ldots$

4.1.1 241-B, C, T, and U Tank Farms . . . . . . . . . . . . 4-4

4.1 .2 241-BX Tank Farm . . . . . . . . . . . . . . . . . . 4-21

$4.1 .3241-$ TX Tank Farm . . . . . . . . . . . . . . . . . . 4-24

4.1 .4241 -BY Tank Farm . . . . . . . . . . . . . . . . . 4-29

$4.1 .5241-\mathrm{S}$ Tank Farm . . . . . . . . . . . . . . . . . 4-32

$4.1 .6241-\mathrm{TY}$ Tank Farm . . . . . . . . . . . . . . . . . 4-35

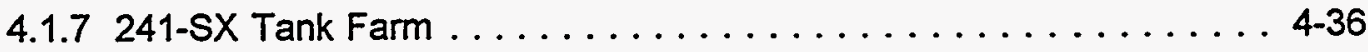

4.1 .8 241-A Tank Farm . . . . . . . . . . . . . . . . . 4 4-40

$4.1 .9241-A X$ Tank Farm . . . . . . . . . . . . . . . . . . 4-42

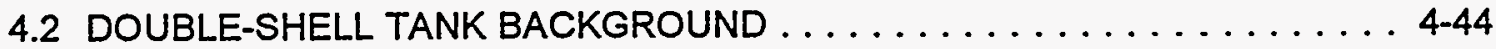

4.2 .1 241-AP Tank Farm . . . . . . . . . . . . . . . 4-47

4.2 .2 241-AN Tank Farm . . . . . . . . . . . . . . . . . . 4-48

4.2.3 241-AW Tank Farm . . . . . . . . . . . . . . . . . 4 4-49

4.2 .4241 -SY Tank Farm . . . . . . . . . . . . . . . . . . 4-50

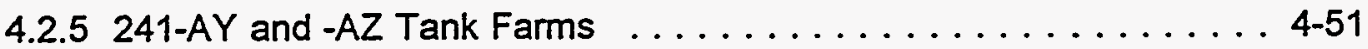

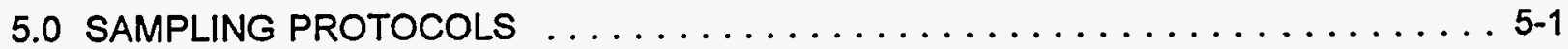

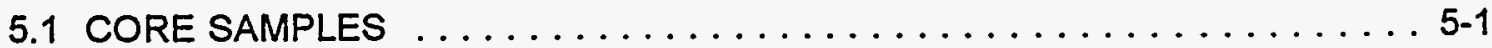

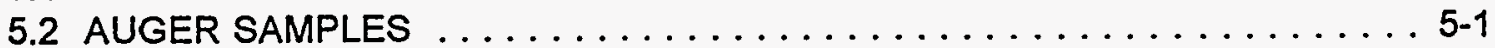

5.3 SUPERNATE AND SOFT SLURRY GRAB SAMPLES . . . . . . . . 5-1

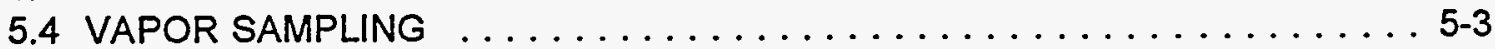

LATA-TCR-9491, Rev. 0 
6.0 ANALYTICAL METHODS SUMMARY $\ldots \ldots \ldots \ldots \ldots \ldots \ldots \ldots \ldots \ldots \ldots \ldots \ldots \ldots$

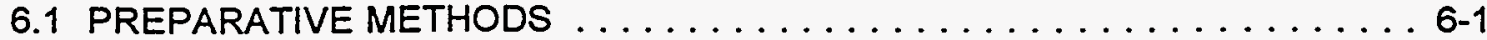

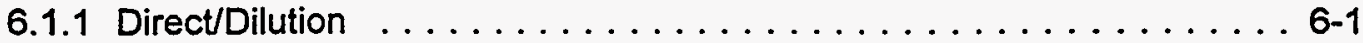

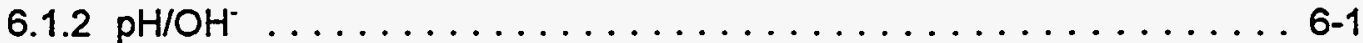

6.1 .3 Acid Digestion $\ldots \ldots \ldots \ldots \ldots \ldots \ldots \ldots \ldots \ldots . \ldots \ldots . \ldots \ldots$ 6.1

6.1 .4 Potassium Hydroxide Fusion . . . . . . . . . . . . . . . . 6-2

6.1.5 Volatile and Semivolatile Organic Compound Extraction ........ 6-2

6.2 ATOMIC SPECTROSCOPIC TECHNIQUES . . . . . . . . . . . . . 6-3

6.2.1 Inductively Coupled Plasma Spectroscopy . . . . . . . . . . . . . 6-3

6.2 .2 Flame AA . . . . . . . . . . . . . . . . . . 6.3

6.2 .3 Graphite Furnace AA . . . . . . . . . . . . . . . . . 6 6-4

6.2.4 Gaseous Hydride Atomic Absorption Spectroscopy . . . . . . . . . . . 6. 6-4

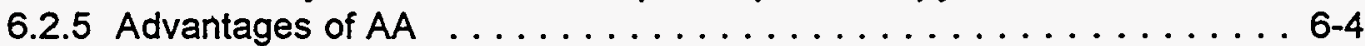

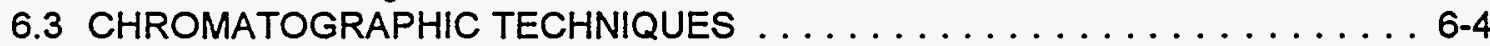

6.3.1 Gas Chromatography . . . . . . . . . . . . . . . . . . . 6 6-5

6.3.2 Mass Spectrometry . . . . . . . . . . . . . . . . . . . . 6 6-5

6.3 .3 Ion Chromatography . . . . . . . . . . . . . . . . 6 6-5

6.3.4 High Performance Liquid Chromatography . . . . . . . . . . . . . 6-6

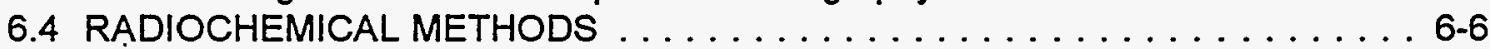

6.4.1 Gas Proportional Detectors . . . . . . . . . . . . . . . 6-6

6.4 .2 Gamma Energy Analysis . . . . . . . . . . . . . . . . . . 6. 6-6

6.4 .3 Alpha Spectrometry . . . . . . . . . . . . . . . . . 6-7

6.4 .4 Liquid Scintillation Counting . . . . . . . . . . . . . . . . 6 6-7

6.5 THERMAL METHODS OF ANALYSIS $\ldots \ldots \ldots \ldots \ldots \ldots \ldots \ldots \ldots$

6.6 PHYSICAL AND RHEOLOGICAL PROPERTIES . . . . . . . . . . . . . 6-8

6.6 .1 Physical Properties . . . . . . . . . . . . . . . . . . . . . 6-8

6.6 .2 Rheological Properties . . . . . . . . . . . . . . . . 6 6-9

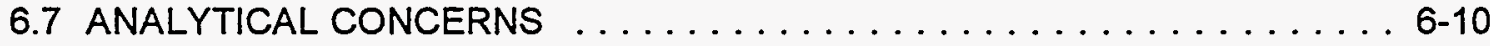

6.7.1 Hold Time Considerations . . . . . . . . . . . . . . . 6-10

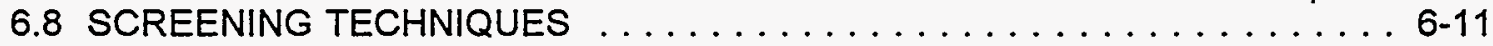

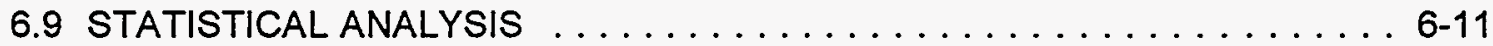

7.0 CHARACTERIZATION DRIVERS $\ldots \ldots \ldots \ldots \ldots \ldots \ldots \ldots \ldots \ldots \ldots \ldots \ldots$

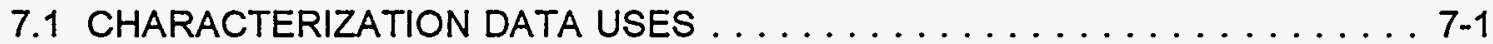

7.1 .1 Resolution of Safety Issues . . . . . . . . . . . . . . . . . .

7.1 .2 Operations and Maintenance . . . . . . . . . . . . . . 7-1

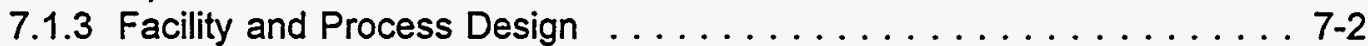

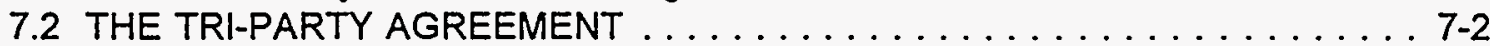

7.3 DATA QUALITY OBJECTIVE PROCESS $\ldots \ldots \ldots \ldots \ldots \ldots \ldots \ldots \ldots$

8.0 TANK CHARACTERIZATION REPORT OVERVIEW $\ldots \ldots \ldots \ldots \ldots \ldots \ldots \ldots$

8.1 TCR ORGANIZATION $\ldots \ldots \ldots \ldots \ldots \ldots \ldots \ldots \ldots \ldots \ldots \ldots \ldots$ 8-1

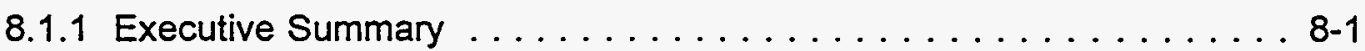

8.1 .2 Introduction . . . . . . . .

8.1.3 Historical Tank Information . . . . . . . . . . . . . . 8-1

8.1.4 Tank Sampling Overview . . . . . . . . . . . . . . . 8-1

8.1.5 Sample Handling and Analytical Scheme . . . . . . . . . . 8-2

8.1.6 Analytical Results and Waste Inventory . . . . . . . . . . . . 8-2

8.1.7 Analytical Results Interpretation . . . . . . . . . . . . . . 8-2

8.1 .8 Statistical Interpretation . . . . . . . . . . . . . . . . 
8.1 .9 Conclusions and Recommendations . . . . . . . . . . . . 8-2 8.1.10 References ...................... 8-3 8.2 CONTEXT FOR INTERPRETATION OF SELECTED TANK ANALYTE

VALUES ......................... 8-3

8.3 TANK SYSTEM DANGEROUS WASTE PERMIT $\ldots \ldots \ldots \ldots \ldots \ldots \ldots \ldots .$.

8.3.1 Waste Characteristics . . . . . . . . . . . . . . . . 8-4

8.3.2 Waste Criteria . . . . . . . . . . . . . . . . . 8-4

8.3.3 Waste Sources $\ldots \ldots \ldots \ldots \ldots \ldots \ldots \ldots \ldots \ldots . \ldots \ldots$ 8-7

9.0 HISTORICAL TANK ESTIMATES $\ldots \ldots \ldots \ldots \ldots \ldots \ldots \ldots \ldots \ldots \ldots \ldots$

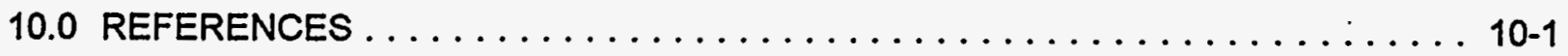

Appendix A. Glossary $\ldots \ldots \ldots \ldots \ldots \ldots \ldots \ldots \ldots \ldots \ldots \ldots \ldots \ldots \ldots \ldots$

LATA-TCR-9491, Rev. 0 iv 


\section{LIST OF FIGURES}

2-1. Location of the Hanford Site $\ldots \ldots \ldots \ldots \ldots \ldots \ldots \ldots \ldots \ldots \ldots \ldots \ldots \ldots \ldots \ldots$

3-1. Hanford Site Processing Timeline . . . . . . . . . . . . . . . . 3-3

3-2. B Plant Cutaway View . . . . . . . . . . . . . . . . . . . 3-5

3-3. PUREX Plant Cutaway View . . . . . . . . . . . . . . . 3-7

3-4. Configuration of a Typical Vault $\ldots \ldots \ldots \ldots \ldots \ldots \ldots \ldots \ldots \ldots . \ldots \ldots$

4-1. Tank Farm Locations within the Hanford Site . . . . . . . . . . . . . 4-2

4-2. Typical Single-Shell Tank (100 Series) Configuration (241-B, $-B X,-C,-T$, and $-U$ ) . . 4-8

4-3. Typical Single-Shell Tank (200 Series) Configuration $(241-B,-C,-T,-U) \ldots \ldots$. . . 4-9

4-4. Typical Single-Shell Tank Configuration (241-BY, $-S$, -TX, and -TY) . . . . . 4-25

4-5. Typical Single-Shell Tank Configuration $(241-A,-A X,-S X) \ldots \ldots \ldots \ldots$. . . . 4-39

4-6. Typical Double-Shell Tank Configuration . . . . . . . . . . . . . . 4-46

5-1. Stainless Steel Core Sampler . . . . . . . . . . . . . . . . . . . 5-2

\section{LIST OF TABLES}

3-1. Hanford Site High-Level Waste Volumes $1944-80 \ldots \ldots$. . . . . . . . . . . . 3-2

3-2. Hanford Site Tank Waste Inventory . . . . . . . . . . . . . . . . . . 3-2

3-3. Average Monthly Waste Generation . . . . . . . . . . . . . . . 3-11

3-4. Hanford Site Evaporator Processing Totals . . . . . . . . . . . . . . 3-12

4-1. 241-B Tank Farm Generic Description. . . . . . . . . . . . . . . 4-4

4-2. 241-B Tank Farm Status . . . . . . . . . . . . . . . . . 4-5

4-3. 241-C Tank Farm Generic Description ... . . . . . . . . . . . . . . 4-10

4-4. 241-C Tank Farm Status . . . . . . . . . . . . . . . . . . . 4-11

4-5. 241-T Tank Farm Generic Description . . . . . . . . . . . . . . . 4-13

4-6. 241-T Tank Farm Status ... . . . . . . . . . . . . . . . . . 4-14

4-7. 241-U Tank Farm Generic Description . . . . . . . . . . . . . . 4-17

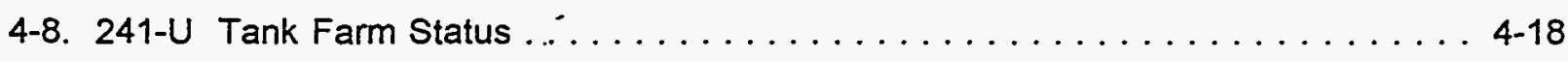

4-9. 241-BX Tank Farm Generic Description . . . . . . . . . . . . . 4-21

4-10. 241-BX Tank Farm Status . . . . . . . . . . . . . . . . . . 4-22

4-11. 241-TX Tank Farm Generic Description . . . . . . . . . . . . . . . . . . . 4-24

4-12. 241-TX Tank Farm Status . . . . . . . . . . . . . . . . . . 4-26

4-13. 241-BY Tank Farm Generic Description . . . . . . . . . . . . . . . . . . 4-29

4-14. 241-BY Tank Farm Status ... . . . . . . . . . . . . . . . . 4-30

4-15. 241-S Tank Farm Generic Description . . . . . . . . . . . . . . . . . 4-32

LATA-TCR-9491, Rev. 0 
4-16. 241-S Tank Farm Status $\ldots \ldots \ldots \ldots \ldots \ldots \ldots \ldots \ldots \ldots \ldots \ldots \ldots \ldots \ldots .33$

4-17. 241-TY Tank Farm Generic Description . . . . . . . . . . . . . . . .

4-18. 241-TY Tank Farm Status . . . . . . . . . . . . . . . . . . . . .

4-19. 241-SX Tank Farm Generic Description $\ldots \ldots \ldots \ldots \ldots \ldots \ldots \ldots \ldots \ldots .4 .36$

4-20. 241-SX Tank Farm Status. . . . . . . . . . . . . . . . . . . . . . . .

4-21. 241-A Tank Farm Generic Description . . . . . . . . . . . . . . . . .

4-22. 241-A Tank Farm Status . . . . . . . . . . . . . . . . . . . 41

4-23. 241-AX Tank Farm Generic Description $\ldots \ldots \ldots \ldots \ldots \ldots \ldots \ldots \ldots .442$

4-24. 241-AX Tank Farm Status $\ldots \ldots \ldots \ldots \ldots \ldots \ldots \ldots \ldots \ldots \ldots \ldots \ldots \ldots \ldots \ldots \ldots .43$

4-25. Double-Shell Tank Farm Generic Description . . . . . . . . . . . . . . 4-44

4-26. 241-AP Tank Farm Status . . . . . . . . . . . . . . . . . . 4-47

4-27. 241-AN Tank Farm Status . . . . . . . . . . . . . . . . . . . . . . 4-48

4-28. 241-AW Tank Farm Status $\ldots \ldots \ldots \ldots \ldots \ldots \ldots \ldots \ldots \ldots \ldots \ldots \ldots \ldots .49 . \ldots \ldots$

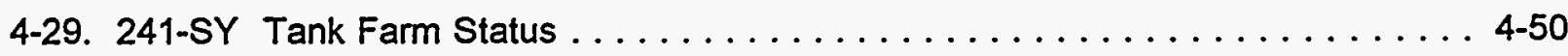

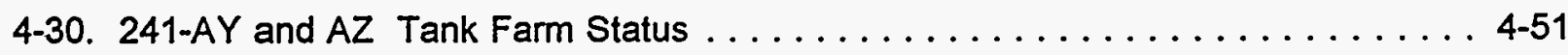

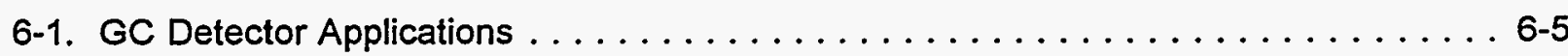

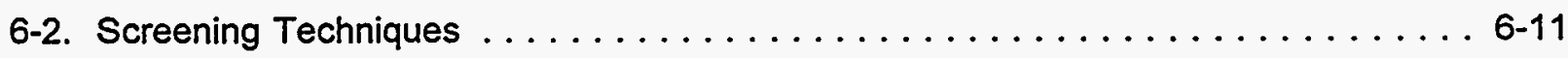

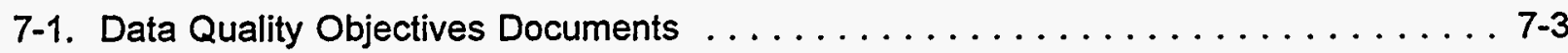

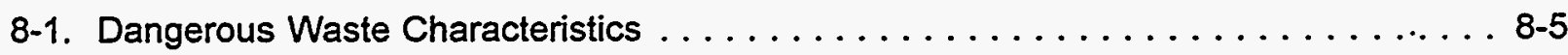

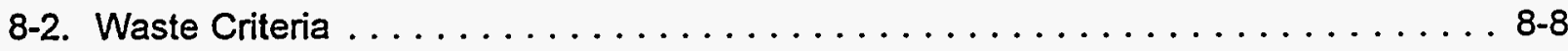

8-3. Waste Sources $\ldots \ldots \ldots \ldots \ldots \ldots \ldots \ldots \ldots \ldots \ldots \ldots \ldots \ldots$ 8.9 


\section{LIST OF TERMS}

$\begin{array}{ll}\text { AA } & \text { atomic absorption } \\ \text { ANOVA } & \text { analysis of variance } \\ \text { AR } & \text { washed PUREX sludge solids } \\ \text { ASF } & \text { ammonia scrubber feed (waste) } \\ \text { ASMD LKR } & \text { assumed leaker } \\ \text { ASTM } & \text { American Society for Testing and Materials } \\ \text { AW } & \text { aging waste } \\ \text { B } & \text { B Plant (waste) } \\ \text { BFSH } & \text { B Plant flush (waste) } \\ \text { BL } & \text { B Plant low-level (waste) } \\ \text { BLEB } & \text { B Plant low-level (waste) } \\ \text { CAM } & \text { continuous air monitoring (alarm) } \\ \text { CARB } & \text { sodium carbonate organic wash (waste) } \\ \text { CASS } & \text { Computer Automated Surveillance System } \\ \text { CC } & \text { complexant concentrate (waste) } \\ \text { CF } & \text { cesium feed (waste) } \\ \text { CLP } & \text { Contract Laboratory Program } \\ \text { CP } & \text { concentrated phosphate (waste) } \\ \text { CSR } & \text { cesium recovery (waste) } \\ \text { CW } & \text { cladding waste } \\ \text { CWHT } & \text { concentrated waste holding tank } \\ \text { CWP } & \text { cladding waste PUREX } \\ \text { CWR } & \text { cladding waste REDOX } \\ \text { DC } & \text { dilute complexed (waste) } \\ \text { DE } & \text { diatomaceous earth } \\ \text { DN } & \text { dilute noncomplexed (waste) } \\ \text { DOE } & \text { U.S. Department of Energy } \\ \text { DQO } & \text { data quality objectives } \\ \text { DRCVR } & \text { dilute receiver (tank) } \\ \text { DSC } & \text { differential scanning calorimetry } \\ \text { DSSF } & \text { double-shell slurry feed } \\ \text { DSS } & \text { double-shell slurry } \\ \text { DST } & \text { double-shell tank } \\ \text { DTA } & \text { differential thermal analysis } \\ \text { DW } & \text { decontamination waste or dangerous waste } \\ \text { EB } & \text { evaporator bottoms } \\ \text { EC } & \text { electron capture (detector) } \\ \text { EDTA } & \text { ethylenediametetraacetic acid } \\ \text { EF } & \text { evaporator feed } \\ \text { EHW } & \text { extremely hazardous waste } \\ \text { EPA } & \text { U.S. Environmental Protection Agency } \\ \text { EVFD } & \text { evaporator feed (tank) } \\ \text { FAA } & \text { flame atomic absorption } \\ \text { FIC } & \text { food Instrument Corporation } \\ \text { FID } & \end{array}$




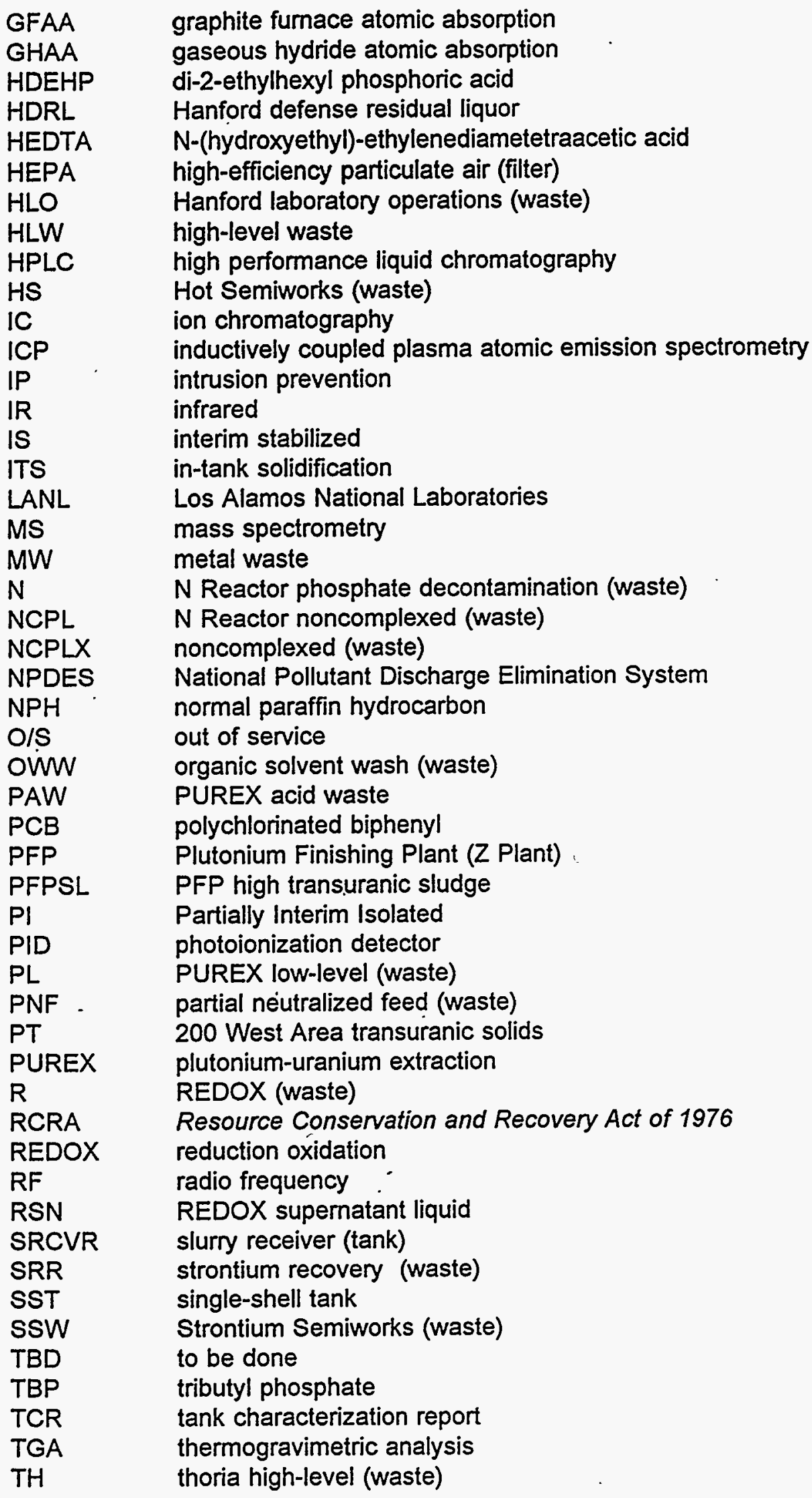

LATA-TCR-9491, Rev. 0 viii 


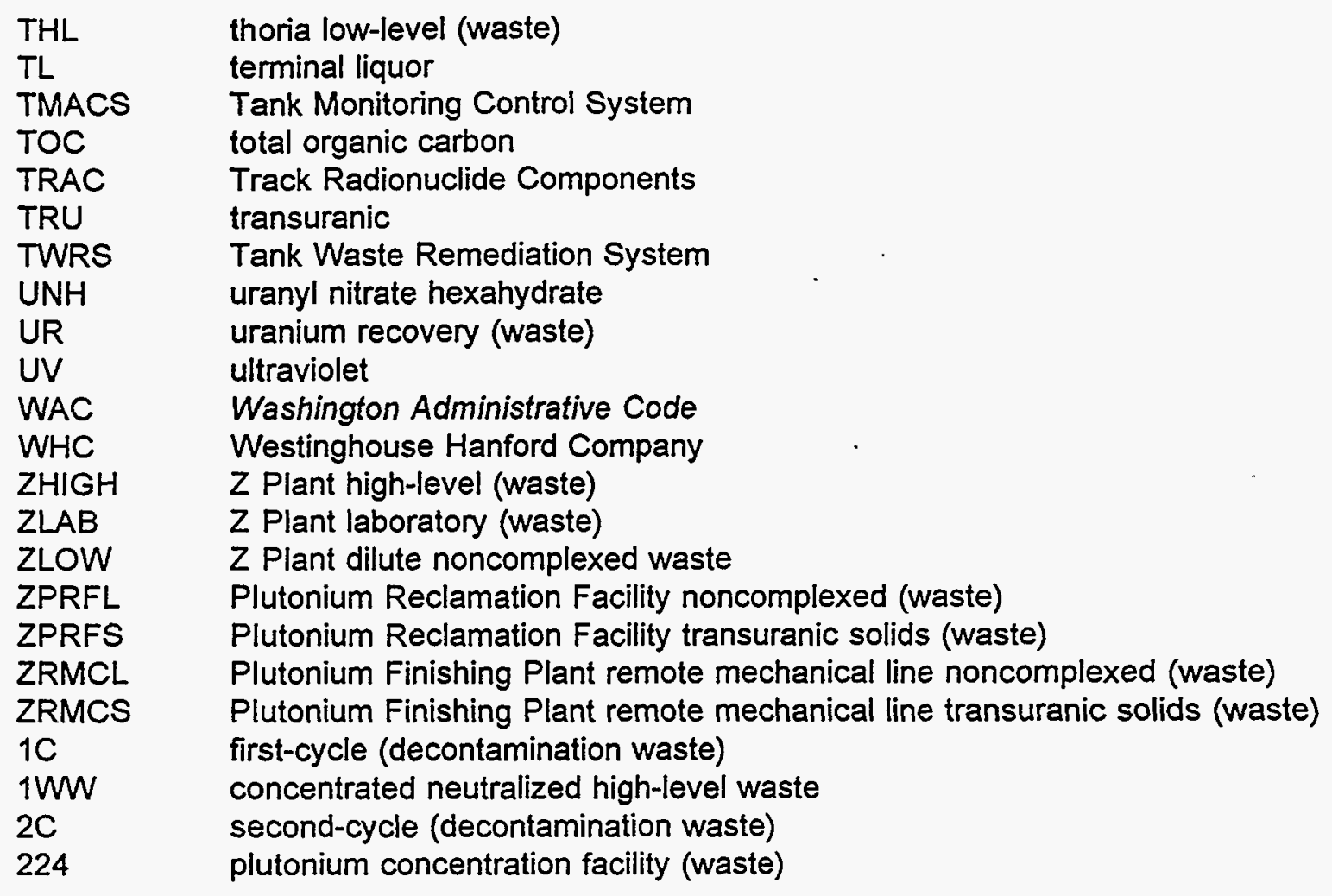




\subsection{INTRODUCTION}

\subsection{PURPOSE}

Characterization of the high-level wastes in the 177 Hanford Site underground storage tanks involves a series of activities that include waste sampling, laboratory analysis, data interpretation, and historical review. The purpose of this reference guide is to provide background information concerning the Hanford Site waste tanks, and to familiarize the reader with issues and concepts generic to the characterization of all tanks. This document does not contain all technical, historical, safety, or management related information about the waste tanks; such information is contained in other, more lengthy reports. It is intended that readers unfamiliar with characterization issues and the associated Tank Characterization Reports will read this Reference Guide initially, and then refer back to it occasionally for questions or clarification about specific issues.

\subsection{SCOPE}

This document provides a broad background of information relating to the characterization of the Hanford Site single-shell and double-shell waste tanks. Topics covered include general descriptions of all tank farms, process and waste generation histories, sampling and analytical methods, and regulatory, safety, and technology development driven characterization needs.

Note: This report is loosely based on a paper prepared by D.S. De Lorenzo and B.C. Simpson, and presented to the American Chemical Society, Hanford Site Waste Tank Characterization (De Lorenzo and Simpson, 1994). 


\subsection{HANFORD SITE HISTORY}

\subsection{PRODUCTION AND FACILITIES HISTORY}

The Department of Energy (DOE) operates the Hanford Site on approximately 1,450 square kilometers (560 square miles) of arid steppe in the southeastern part of Washington State. The isolated, sparsely populated location near the Columbia River was selected in 1942 as the site for the first industrial-sized operations to manufacture plutonium as part of the Manhattan Project, the secretive World War II program that produced the worid's first nuclear weapons.

Selection criteria developed in 1942 for the selection of a Manhattan Project plutonium production site called for a large remote tract of land with room for a manufacturing area measuring at least 19.3 by 25.7 kilometers ( 12 by 16 miles), space for laboratory facilities at least 12.9 kilometers ( 8 miles) from the nearest reactor or processing plant, and abundant supplies of water and electricity (Gerber, 1992). Hence, production reactors were located along the Columbia River as it flows eastward across the northern part of the Hanford Site and curves to the south (100 Area), processing plants and associated facilities (e.g., waste tanks) were located on a plateau near the center of the Site (200 Area), and fuel fabrication buildings, laboratories, and other support facilities were located near the site's southern boundary ( 300 Area). The location of the Hanford Site is shown in Figure 2-1.

The initial 30-month, war-time construction period saw the building of the first three production reactors $(B, D$, and $F$ ), the first plutonium separations plants, and 64 high-level waste tanks (Gerber, 1992). These single-shell tanks (comprising the $B, C, T$, and $U$ Tank Farms) were built near the locations of four planned chemical processing facilities. Of the four plants $(B, C, T$ and $U$ ), only three were completed during this initial phase $(B, T$, and $U$ ); and only two ( $B$ and $T$ ) were used in the first operations to separate plutonium from uranium fuel irradiated at Hanford Site production reactors. The first separations process was based on bismuth phosphate. In this process, bismuth phosphate was used to precipitate plutonium from an acidic solution of dissolved fuel. The resulting wastes, directed to the high-level waste tanks, carried away much of the original uranium and most of the fission products. The plutonium solution was then sent to additional processing facilities where it was further purified by coprecipitation with lanthanum fluoride (Jungfleisch, 1984).

The conclusion of World War II brought a period of uncertainty regarding the future operations of the Hanford Site, or Hanford Engineer Works as it was then called (Gerber, 1992). However, 1947 marked the beginning of the first of a number of periods of rapid expansion of the Site as the Cold War began and intensified. Soon after the transfer of the U.S. atomic complex to the control of the newly formed Atomic Energy Commission (a forerunner of the DOE) the $H$ and DR Reactors were built, and the C Plant was completed for use as a pilot facility for new chemical separations processes under development. The Plutonium Finishing Plant ( PFP or $Z$ Plant) began operations in 1949. At this facility, the final product of chemical separations processing, a wet paste of plutonium nitrate, was converted into plutonium metal. Before completion of the PFP, plutonium nitrate was sent to laboratories at Los Alamos, New Mexico, for finishing. The additional waste generation of the new facilities, along with increasing production rates at the original facilities, required more storage space in the high-level waste tanks. By 1946, half of the original tanks were filled to capacity and the remaining tanks were about $40 \%$ full. From 1947-49, 42 additional single-shell tanks (comprising the BX, BY, and TX Tank Farms) were built. 
Figure 2-1. Location of the Hanford Site.

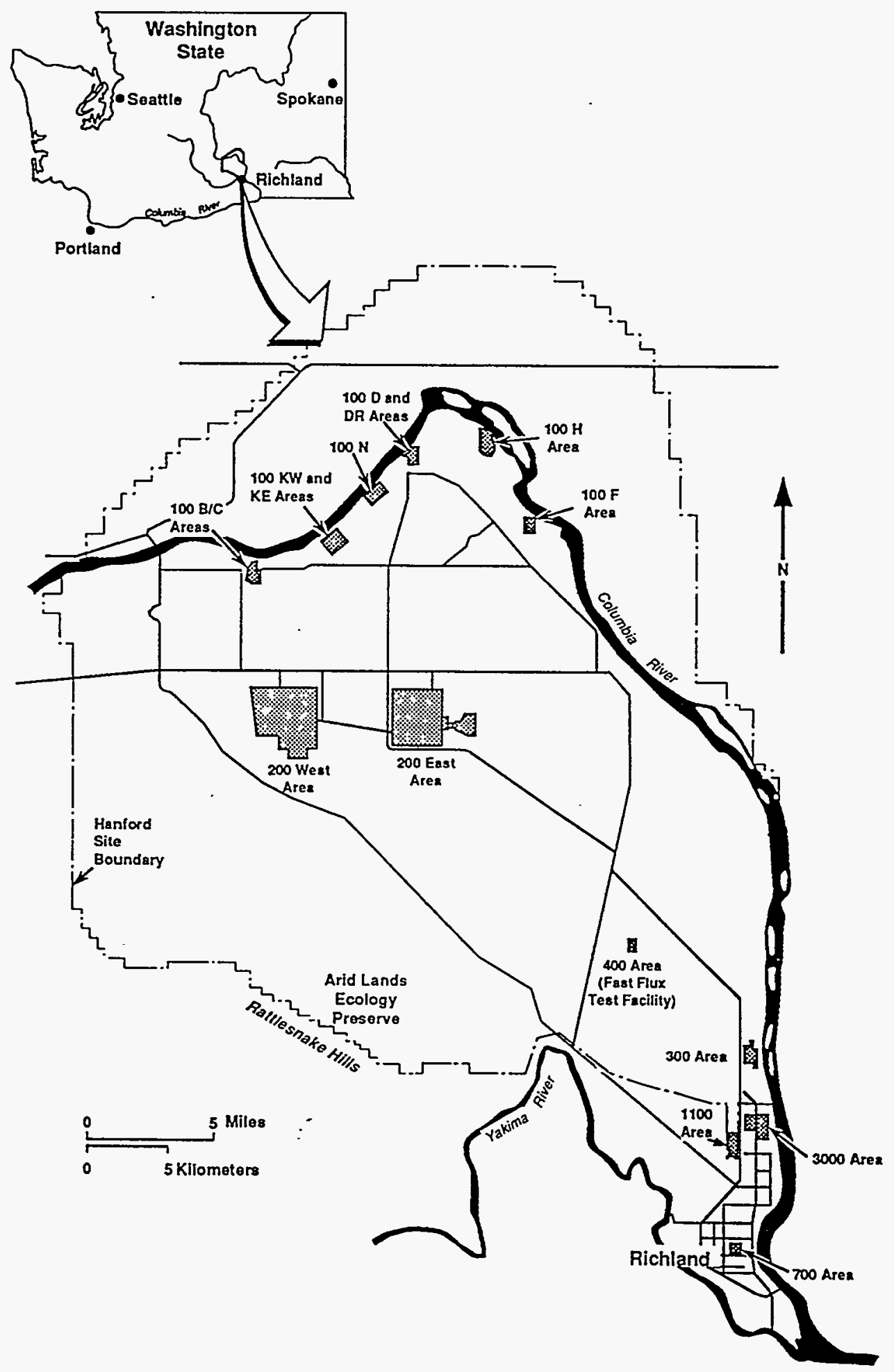


Another construction surge occurred from 1950-52 following the Soviet Union's first detonation of an atomic bomb and the U.S. decision to pursue development of a hydrogen bomb. The REDOX Plant, using a new, more efficient, solvent extraction process, began operation in 1952. The REDOX (reduction oxidation) process used a more efficient continuous processing method, rather than batch processing employed in the bismuth phosphate method. The solvent process, based on methyl isobutyl ketone (hexone), also recovered both plutonium and uranium (Agnew, 1994a). In 1953, overall plutonium production increased by $38 \%$ from the previous year, with $88 \%$ of the product processed in the REDOX Plant (Gerber, 1994); T Plant accounted for the remaining $12 \%$. A short supply of high-grade uranium made imperative a new operation to recover uranium-laden waste from bismuth phosphate processing that had been directed to highlevel waste tanks since T Plant began operations (Gerber, 1994). A solvent extraction process using tributyl phosphate (TBP) and normal paraffin hydrocarbon (NPH) was operated at $U$ Plant from 1952-57. Single-shell tanks were sluiced with the waste directed to process vaults. In the vaults, acid was added and the waste was processed through $U$ Plant. The uranium recovery process created a large amount of waste, about triple the amount sent to processing, resulting in a greater volume than could be stored in the tank farms. This led to a scavenging program to remove soluble ${ }^{137} \mathrm{Cs}$ through precipitation, thus allowing the waste to be discharged to the ground (see Section 3.2.3 for a further discussion of ferrocyanide scavenging). Also during this period of expansion, $C$ Reactor was built, along with 18 more single-shell tanks (the $S$ and TY Tank Farms).

The mid-1950's saw the addition of the KE and KW Reactors, new reactors with much greater capacities than the earlier reactors. This brought the number of Hanford Site production reactors operating in the 100 Area along the Columbia River to eight. Also, 21 more single-shell tanks (the SX and A Tank Farms) were constructed. In 1955, a new process was begun at the large new Plutonium-Uranium Extraction Plant (PUREX or A Plant) following a pilot run at the $C$ Plant (Anderson, 1990). The PUREX process used a solvent extraction method based on TBP and NPH rather than potentially explosive hexone (Gerber, 1994).

The time period 1956-63 was the time of peak production for the Hanford Site as Cold War tensions intensified (Gerber, 1992). The PUREX. Plant demonstrated such an overwhelming production capacity that a plan was abandoned to bring all four chemical separations plants on line (B Plant ceased operating in 1952; T Plant was subsequently shut down in 1956). In 1958, the PUREX Plant processed $79 \%$ of the plutonium product produced that year. By October 1960, the PUREX Plant reached the point of having processed 20,000 metric tons $(22,000$ short tons) of fuel, surpassing the combined total of B Plant, T Plant, and REDOX Plant (Gerber, 1993). In 1967, B Plant was reconfigured for a fractionation process which separated heat generating ${ }^{90} \mathrm{Sr}$ and ${ }^{137} \mathrm{Cs}$ isotopes from PUREX waste, and a number of other wastes stored in the tank farms. Also in 1967, the REDOX Plant was shut down as PUREX became the sole operating separations process facility. The -PUREX Plant operated until 1972 when it was shut down for 11 years. One of the few new facilities added during the peak production years was $N$ Reactor, a plant that combined plutonium production with the commercial generation of electricity (Gerber, 1992). It also used a closed-circulating-water cooling system, rather than single-pass systems employed in earlier reactors. The last four single-shell tanks (comprising the AX Tank Farm) were built from 1963-64. Major new capabilities were added at the Plutonium Finishing Plant. The Recuplex operation, a method of reclaiming plutonium from process scraps and waste, had been operating at the PFP since 1955. A criticality in 1962 closed the operation, which was then replaced in 1964 by the larger Plutonium Reclamation Facility. 
In 1983, PUREX resumed operations as a part of the overall U.S. defense mission. During the years the plant was shut down, many modifications were made, especially in terms of waste minimization and waste management, in response to changing Hanford Site policies. The plant was placed on standby status in 1990 and the DOE issued closure orders in December 1992. The plant is in the process of being deactivated (Gerber, 1994).

The present objectives of the DOE and its contractors have changed significantly from the weapons production and testing missions of the past to waste management, environmental remediation, and energy and environmental research. Plans guiding the operation of the Hanford Site anticipate the eventual retrieval, treatment, and disposal of the contents of the high-level waste tanks, and the remediation and restoration of the surrounding lands. This clean-up effort is the largest project of its type ever undertaken, and the DOE has designated the Hanford Site as the flagship of the agency's nuclear waste remediation program (Gerber, 1992). These new missions require accurate knowledge of stored wastes to guide technology development and to satisfy the compliance criteria for the various regulatory agencies that oversee activities at the nation's DOE sites.

\subsection{HANFORD SITE TANK FARMS}

Since 1944 the production of defense related materials at the Hanford Site has resulted in the large-scale generation of solid and liquid radioactive mixed waste. The chemical processing of irradiated uranium fuels has generated alkaline slurries containing heavy metals, organic and inorganic salts, uranium, plutonium, and mixed fission products (Anderson, 1990). While cooling water, condensates, and other low-level wastes from processing operations were discharged to the ground, high-level wastes were placed in the large, steel, underground tanks for temporary storage. As improvements were developed in process operations over the years, improvements and modifications were also made to the high-level waste tanks as more tanks were built to store the increasing volume of waste.

No process wastes have been added to the 149 single-shell tanks since November 1980 . As a result of leakage at the single-shell tank farms, double-shell tank construction was started in the late 1960's. No tank leaks were reported at the Hanford Site before the early 1950's, although some pipes and fittings made from materials other than stainless steel leaked as early as 1945 (Gerber, 1992). The first significant confirmed tank leak $(208,000 \mathrm{~L}$ or 55,000 gal) occurred in 1956 at Tank 241-U-104. The largest known leak from a Hanford single-shell tank occurred in 1973, when $435,000 \mathrm{~L}(115,000 \mathrm{gal})$ escaped from $241-\mathrm{T}-106$. Currently, 67 single-shell tanks are listed as assumed leakers (tank surveillance data indicate a loss of liquid attributed to a breach of integrity), but no double-shell tanks have leaked (Hanlon, 1994).

Twenty-eight double-shell tanks (comprising the AN, AP, AW, AY, AZ, and SY Tank Farms) were placed into service beginning in 1970. The double shell was designed to provide an extra barrier against the release of radioactive waste (Anderson, 1990). They also include recovery capabilities for any liquid that might collect in the annular space between the liners. The double-shell tanks are Resource Conservation and Recovery Act (RCRA) interim status facilities that continue to receive waste. This brings the present total number of high-level waste tanks at the Hanford Site to 177. New double-shell tanks are planned to aid in waste management and waste remediation operations. 
WHC-SD-WM-TI-648 REV 0

\subsection{WASTE TYPES AND MANAGEMENT PRACTICES}

\subsection{WASTE DESCRIPTION AND GENERATION}

The majority of wastes stored in the Hanford Site's underground waste tanks are radioactive slurries generated by irradiated uranium fuel reprocessing using one of the following processes: the bismuth phosphate process, the reduction oxidation (REDOX) process, the plutonium-uranium extraction (PUREX) process, the tributyl phosphate (TBP) process, and the B Plant waste fractionation process (Anderson, 1990). Wastes were generated in smaller volumes from research and development programs, laboratory processes, and Plutonium Finishing Plant operations (Winters et al., 1990). Since 1944, more than 1,510,000 kL (400 million gal) of high-level waste have been generated by these operations and various other sources (Table 3-1 presents a summary and general breakdown of the inventory)(Agnew, 1994a). More than 1,140,000 kL (300 million gal) were directed to the Hanford Site's single-shell and double-shell tanks. This amount has been reduced to approximately $231,000 \mathrm{~kL}$ ( 61 million gal) by evaporation/concentration, and by decanting dilute wastes to the ground (see Table 3-2).

All of the fuel processing methods generated acidic waste streams. Sodium hydroxide or calcium carbonate was added to the waste before being transferred to the tanks to neutralize the acid, minimizing tank corrosion. The tanks currently contain moderately to strongly alkaline solutions, with pH values of up to 14. Additional postprocessing of some of the wastes to recover plutonium and uranium, or to reduce the volume of high-level waste, have resulted in the addition of ferrocyanide and some organic compounds listed as hazardous (Anderson, 1990).

The tanks now contain a mixture of salt cake, liquid, and sludges with both radioactive and hazardous components. A current inventory overview is presented in Table 3-2. Sludge consists primarily of solids (hydrous metal oxides) precipitated from the neutralization of acid wastes. Salt cake consists of the various salts formed from the evaporation of water from the waste. Liquids exist as supernatant (liquid above solids) and interstitial liquid (liquid filling the void between solids) in the tanks. These waste types do not necessarily exist as discrete layers, but are intermingled to different degrees. Some sludges and salt cake may contain interstitial liquids and be relatively soft, while others are drier and harder.

The waste is mostly inorganic. It consists primarily of sodium hydroxide; sodium salts of nitrate, nitrite, carbonate, aluminate, and phosphate; and hydrous oxides of aluminum, iron, and manganese. The radioactive components consist primarily of mixed long-lived fission product radionuclides such as ${ }^{90} \mathrm{Sr},{ }^{137} \mathrm{Cs}$, and actinide elements such as uranium, plutonium, and americium. Complexed waste contains the chelating agents EDTA and HEDTA. There may also be detectable halogenated and nonhalogenated organic compound contamination. The mixed waste in some tanks may have detectable amounts of heavy metals such as lead, chromium, and cadmium (Bell, 1993).

A summary of the fuel reprocessing methods, their associated plants and resultant waste streams directed to the tanks (including common waste stream identifiers) follows. A timeline depicting when the various processes were in operation is presented in Figure 3-1. Additional terms and definitions can be found in Appendix A - Glossary. 
Table 3-1. Hanford Site High-Level Waste Volumes 1944-80.

\begin{tabular}{|c|c|c|}
\hline Processor Facility & Generation Totals & $\begin{array}{l}\text { Percent } \\
\text { Contribution } \\
\text { to Total }\end{array}$ \\
\hline Bismuth Phosphate & $\begin{array}{l}369,000 \mathrm{~kL} \\
(97,600 \mathrm{kgal})\end{array}$ & $25.8 \%$ \\
\hline Tributyl Phosphate & $\begin{array}{l}215,000 \mathrm{~kL} \\
(56,700 \mathrm{kgal})\end{array}$ & $15.0 \%$ \\
\hline PUREX & $\begin{array}{l}316,000 \mathrm{~kL} \\
(83,600 \mathrm{kgal})\end{array}$ & $22.1 \%$ \\
\hline REDOX & $\begin{array}{l}155,000 \mathrm{~kL} \\
(40,900 \mathrm{kgal})\end{array}$ & $10.8 \%$ \\
\hline B Plant Fractionation & $\begin{array}{l}197,000 \mathrm{~kL} \\
(52,000 \mathrm{kgal})\end{array}$ & $13.8 \%$ \\
\hline Ferrocyanide Scavenging & $\begin{array}{l}168,000 \mathrm{~kL} \\
(44,500 \mathrm{kgal})\end{array}$ & $11.8 \%$ \\
\hline Plutonium Finishing Plant (1974-88) & $\begin{array}{l}6,060 \mathrm{~kL} \\
(1,600 \mathrm{kgal})\end{array}$ & $0.4 \%$ \\
\hline Strontium Semiworks & $\begin{array}{l}3,030 \mathrm{~kL} \\
(800 \mathrm{kgal})\end{array}$ & $0.2 \%$ \\
\hline Total: & $\begin{array}{l}1,430,000 \mathrm{~kL} \\
(378,000 \mathrm{kgal})\end{array}$ & $100 \%$ \\
\hline
\end{tabular}

Source: (Agnew, 1994a)

Table 3-2. Hanford Site Tank Waste Inventory.

\begin{tabular}{|c|c|c|}
\hline $\begin{array}{l}\text { Waste Tank Inventory } \\
\text { (As of March 31,1994) }\end{array}$ & $\begin{array}{l}\text { Waste } \\
\text { Volume }\end{array}$ & $\begin{array}{l}\text { Percent Contribution } \\
\text { to Total Inventory }\end{array}$ \\
\hline $\begin{array}{r}\text { Single-Shell Tanks } \\
\text { Liquids } \\
\text { Solids }\end{array}$ & $\begin{array}{cc}2,180 \mathrm{~kL} & (575 \mathrm{kgal}) \\
134,000 \mathrm{~kL} & (35,500 \mathrm{kgal})\end{array}$ & $\begin{array}{r}0.9 \% \\
57.5 \%\end{array}$ \\
\hline Total & $136,000 \mathrm{~kL} \quad(36,100 \mathrm{kgal})$ & - \\
\hline $\begin{array}{r}\text { Double-Shell Tanks } \\
\text { Liquids } \\
\text { Solids }\end{array}$ & 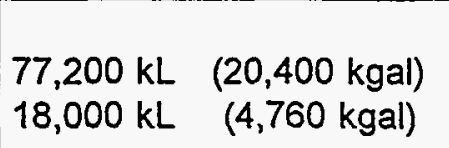 & $\begin{array}{c}33.9 \% \\
7.7 \%\end{array}$ \\
\hline Total & $95,200 \mathrm{~kL} \quad(25,200 \mathrm{kgal})$ & - \\
\hline Total Inventory & $231,000 \mathrm{~kL} \quad(61,300 \mathrm{kgal})$ & $100 \%$ \\
\hline
\end{tabular}

Source: (Hanlon, 1994) 


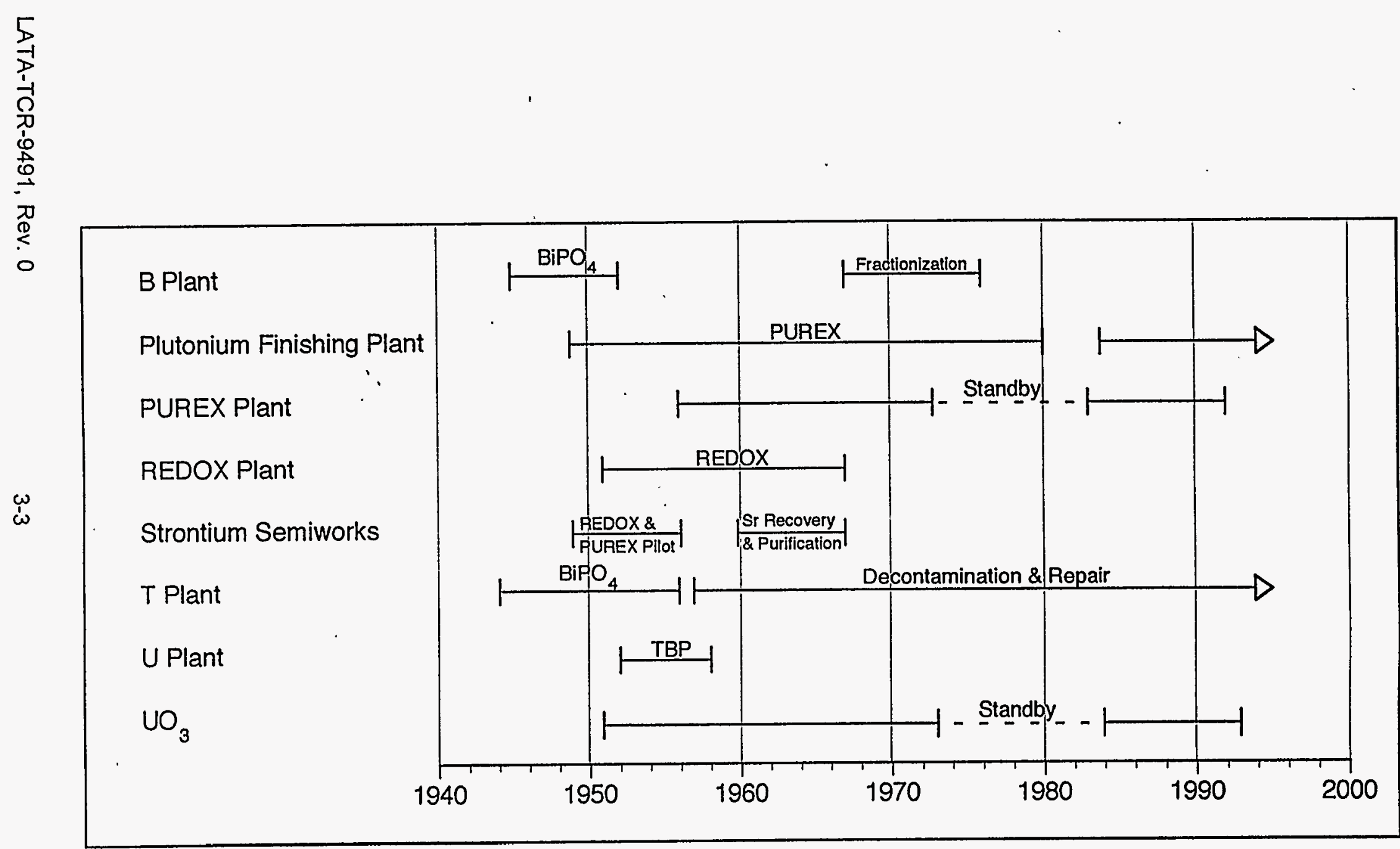




\subsubsection{Bismuth Phosphate Process}

The bismuth phosphate process was first used in T Plant starting in December 1944, and in B Plant in April 1945 generating a total of nearly $371,000 \mathrm{~kL}$ (98 million gal) of high-level waste (Agnew 1 1994a). The bismuth phosphate process is based on the principle that $\mathrm{BiPO}_{4}$ is similar in crystal structure to plutonium phosphate. Thus, the process manipulated the valence state of ${ }^{239} \mathrm{Pu}$ so that it was alternately carried, or left behind in bismuth phosphate solutions (Gerber, 1994). In the first step of this process, aluminum fuel jackets were removed from irradiated fuel slugs using nitric acid. Next, the ${ }^{239} \mathrm{Pu}$ was separated from most of the uranium. About $90 \%$ of the fission products were extracted in the metal waste. The third step, decontamination, was carried out in two cycles. The recovered plutonium was then sent to other facilities for further concentration and purification (Gerber, 1993). Waste streams included: metal waste (MW), firstcycle decontamination waste $(1 \mathrm{C})$, second-cycle decontamination waste $(2 \mathrm{C})$, cladding waste (CW), and plutonium concentration facility waste (224)(Anderson, 1990; Agnew, 1994a).

T Plant. This plant was the first full-scale plutonium separations plant to operate at the Hanford Site (1945-50). Since 1957, T Plant has been used as a decontamination and repair facility (Anderson, 1990). The $T$ Plant waste stream from equipment decontamination, decontamination waste, is designated (DW) and continues to be directed to the Hanford Site's double-shell tanks (Bell, 1993). Part of the facility was modified in 1978 for storage of pressurized water reactor core fuel assemblies.

B Plant. B Plant operated using the bismuth phosphate process until 1952 (Anderson, 1990). The plant was retrofitted in the 1960's to remove cesium and strontium from wastes (see B Plant Fractionation below for a further discussion of this process). A cutaway view of B Plant is presented in Figure 3-2.

\subsubsection{Tributyl Phosphate (TBP) Uranium Recovery Process}

U Plant. This plant originally built as a bismuth phosphate process facility, was modified to recover uranium from bismuth phosphate waste. Metal waste (MW) was mined from tanks in the B, C, BX, BY, T, and TX Tank Farms by sluicing. The waste was then dissolved in nitric acid in process vaults, and then reprocessed in B Plant with a solvent extraction process based on TBP and NPH (Agnew, 1994a). U Plant waste, known as TBP or UR, totaled 215,000 kL (56.7 million gal)(Agnew, 1994a). The main canyon of $U$ Plant currently stores used equipment.

\subsubsection{Reduction Oxidation (REDOX) Process}

REDOX high-level waste was more concentrated in radionuclides than the bismuth phosphate process wastes, and the concentrations of heat-generating radionuclides in this waste stream increased through process improvements. This solvent extraction process, based on methyl isobutyl ketone (hexone), produced both a plutonium product stream and uranyl nitrate hexahydrate $(\mathrm{UNH})$, a uranium product stream complexed in nitric acid. The UNH was sent to another facility to be converted into uranium trioxide powder that was then shipped off site (Gerber, 1993). Irradiated fuel slugs were dissolved into an aqueous aluminum nitrate solution. The valence states of the dissolved plutonium and uranium were then manipulated so that they were extracted or salted out of the solution into an organic phase consisting mainly of methyl isobutyl ketone (hexone) (Agnew, 1994a). Waste from the REDOX (or S Plant) was directed to storage tanks in the $S, S X$, and $U$ Tank Farms. 
WHC-SD-WM-TI-648 REV O

Figure 3-2. B Plant Cutaway View.

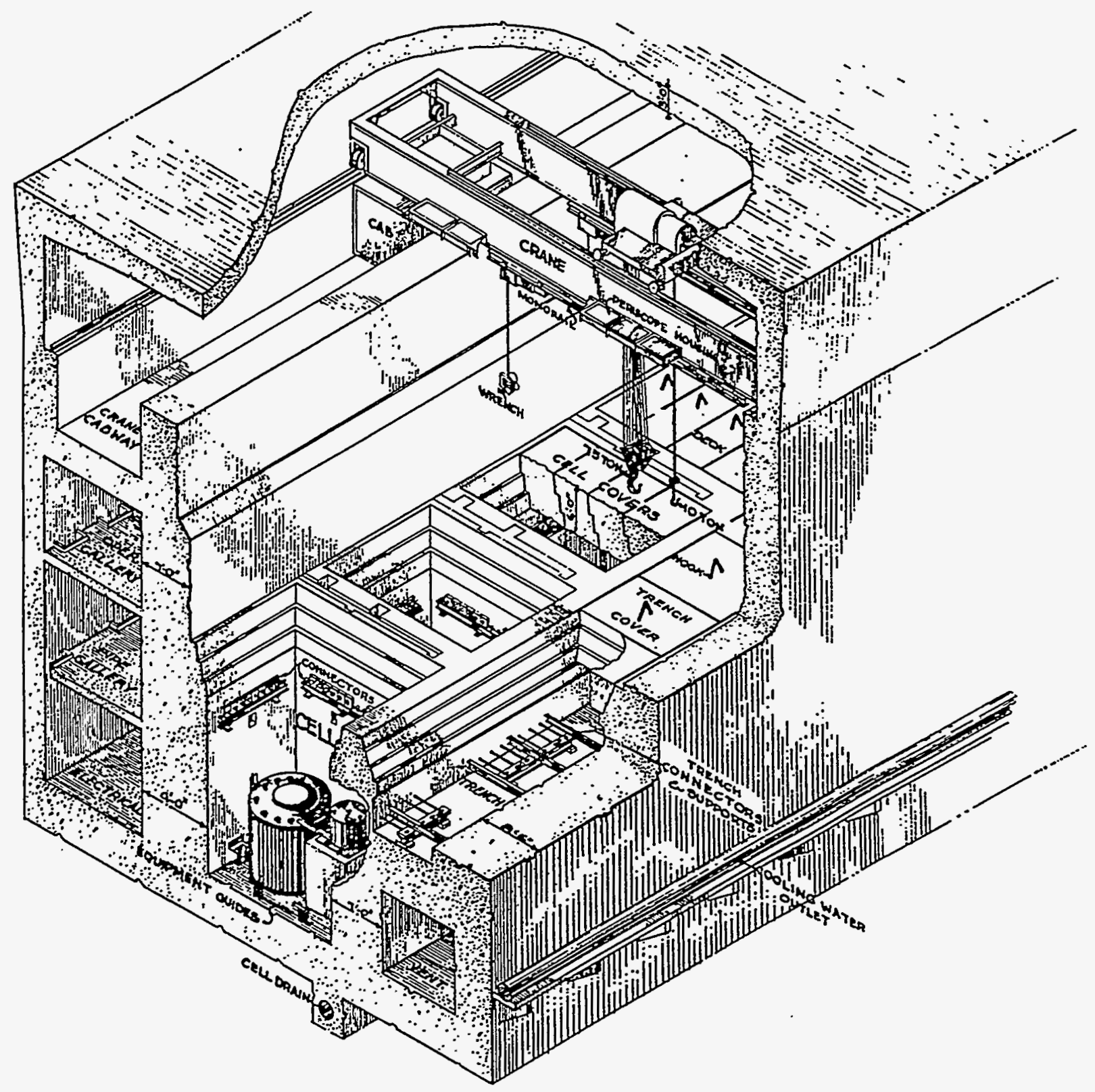


REDOX waste streams consisted of the following: REDOX process waste resulting from the dissolution of aluminum and zircaloy fuel element cladding, REDOX cladding waste (CWR), REDOX high-level waste (R), and REDOX supernatant liquid (RSN) (Agnew, 1994a).

\subsubsection{Plutonium-Uranium Extraction (PUREX) Process}

The PUREX campaigns, and subsequent campaigns to remove strontium from PUREX waste, produced some of the most complicated combination of wastes produced at the Hanford Site (Agnew, 1994a). This solvent extraction method, utilizing an organic phase mixture of NPH and TBP contacting an aqueous nitric acid solution of plutonium and uranium, was much more efficient than REDOX. The nitric acid used at PUREX could be partially decontaminated and reused, as opposed to the aluminum nitrate used at REDOX (Gerber, 1994). Solutions were moved through electrically pulsed columns, rather than the packed columns at REDOX. Not only were the chemicals more efficient, but they had a higher flash point than the hexone used at REDOX so that no components of the PUREX Plant had to be built to be explosion proof (Gerber, 1994). A cutaway view of the PUREX Plant is presented in Figure 3-2.

Two limited campaigns were conducted to process thorium for recovery of the fissionable isotope ${ }^{233} U$, the first in 1966 and second in 1971. The waste from these campaigns was similar to waste from other PUREX campaigns, except it contained residual thorium and ${ }^{233} U$ (U.S. Department of Energy, 1987).

PUREX had the following waste streams associated with it: cladding waste (CWP), organic solvent wash waste (OWW), neutralized PUREX acid waste (PAW), low-level waste (PL), aging waste (AW), organic wash waste using sodium carbonate (CARB), cesium feed (CF), concentrated neutralized high-level waste (1WW), ammonia scrubber feed (ASF), thoria highlevel (TH), and thoria low-level (THL)(Agnew, 1994a; Brevick et al., 1994a).

B Plant Fractionation. This second mission for B Plant separated heat generating ${ }^{{ }^{\circ}} \mathrm{Sr}$ and

${ }^{137} \mathrm{Cs}$ isotopes from PUREX acid waste, PUREX sludges sluiced from the $A$ and $A X$ Tank Farms, and a variety of neutralized supernatant wastes from the tank farms from 1967-72 (Agnew, 1994a). From 1968-72 the PUREX acidified waste (PAW) was sent directly to B Plant for fractionation. The ${ }^{90} \mathrm{Sr}$ was separated using a di-2-ethylhexyl phosphoric acid (HDEHP) solventextraction process. This method used various complexing agents (EDTA, HEDTA, and citrate) to prevent transition metal extraction. The ${ }^{137} \mathrm{Cs}$ isotope was purified by ion exchange. Both ${ }^{90} \mathrm{Sr}$ and ${ }^{137} \mathrm{Cs}$ were later converted to fluoride and chloride salts, respectively, and encapsulated in the Waste Encapsulation and Storage Facility (Winters et al., 1990).

Wastes generated were dẹsignated as follows: washed PUREX sludge solids (AR), waste from PUREX acidified waste (B), low-level waste from all operations (BL), waste from cesium recovery from supernatants (CSR), strontium recovery waste from sluiced PUREX sludge (SRR), and B Plant flush (BFSH) (Agnew, 1994a; Brevick et al., 1994a). 


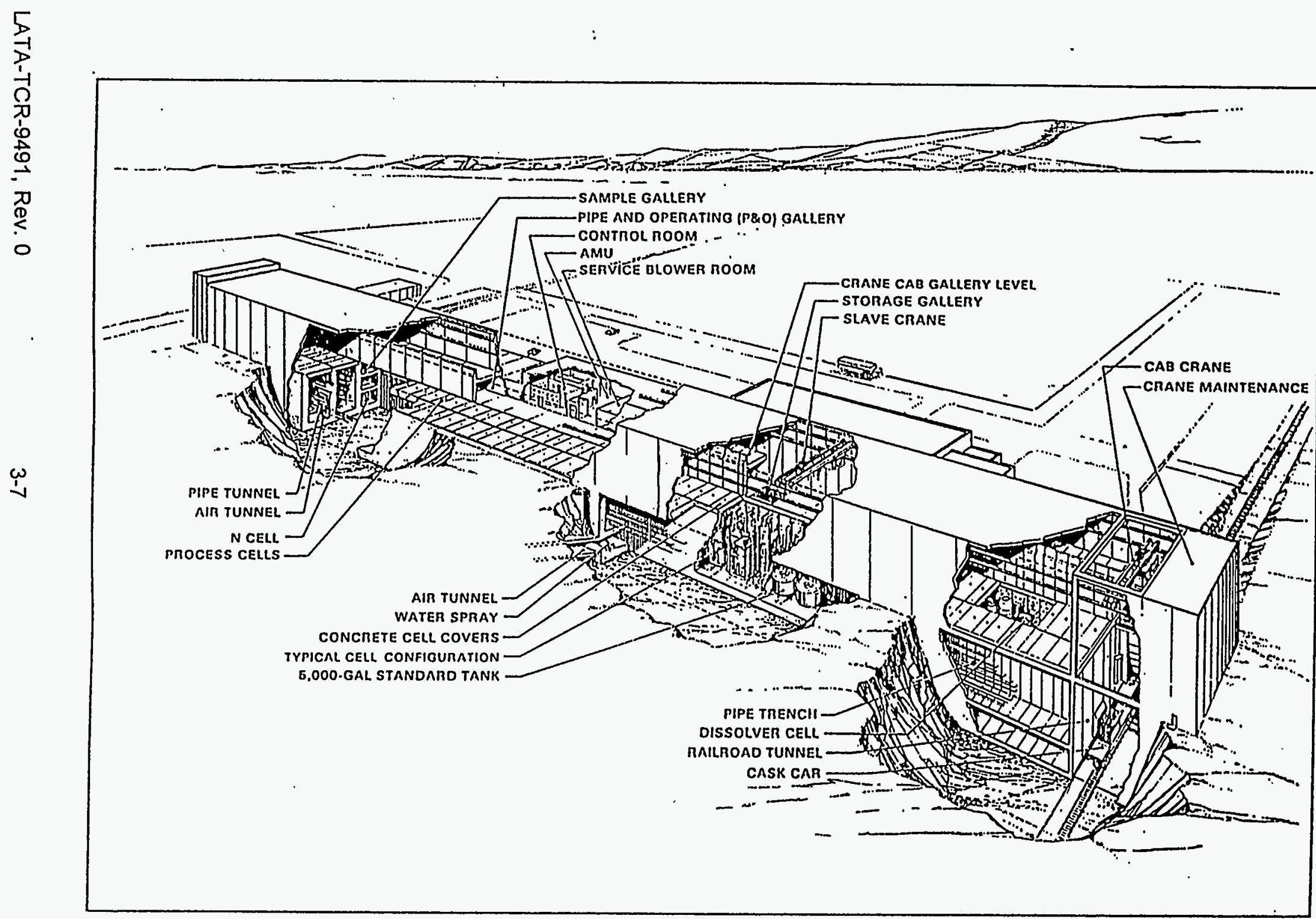

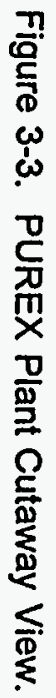




\subsubsection{Other Processes}

Plutonium Finishing Plant. At the Plutonium Finishing Plant (PFP or Z Plant), a TBP solvent extraction process was used to further purify plutonium product from the processing plants and from plutonium scrap (Winters et al., 1990). Plutonium nitrate was converted to plutonium oxide and then smelted to metal in a remote mechanical processing line.

Wastes from $\mathrm{Z}$ Plant contained low concentrations of plutonium and other transuranic elements, but were high in metallic nitrates. The plant's waste streams are identified as follows: dilute noncomplexed waste from Z Plant (ZLOW), Z Plant high-level waste (ZHIGH), PFP hightransuranic sludge (PFPSL), Z Plant lab waste (ZLAB), Plutonium Reclamation Facility transuranic solids (ZPRFS), Plutonium Reclamation Facility noncomplexed waste (ZPRFL), PFP remote mechanical line transuranic solids (ZRMCS), and PFP remote mechanical line dilute noncomplexed waste (ZRMCL)(Koreski, 1994; Brevick et al., 1994a).

C Plant (Hot or Strontium Semiworks). A pilot plant for separations processes and equipment development, C Plant was retired in 1967 (Ballinger and Hall, 1991). A relatively small amount of waste was generated totaling $3,040 \mathrm{~kL}(803,000$ gal); however, they are characteristically high in ${ }^{80} \mathrm{Sr}$ content (Agnew, 1994). Its waste streams were as follows: hot semiworks (HS), strontium semiworks (SSW), and fission products waste (FP).

Vaults. Several smaller waste treatment facilities, known as vaults, were built near the tank farms to settle, evaporate, neutralize and condition plant wastes to reduce the waste volumes directed to the tanks and to make the wastes alkaline to minimize corrosion (Bergmann, 1990). Chemical additions and agitation could be performed in the vaults prior to the waste being transferred. Specific wastes from tanks could be pumped temporarily to the vaults and the wastes could be directed to desired tanks or processing facilities later. In a number of instances, wastes were sent to the single-shell tanks and then later recovered for treatment. Vauit configurations vary, but generally consist of a segmented, underground, concrete structure with a steel tank in each segment interconnected with piping. Equipment is accessed through concrete cover blocks at ground level. The vaults are also close to their related tank farms and processing plants; the AR, BXR, and CR Vaults are in 200 East, while the TXR, UR, and WR Vaults are in 200 West. The configuration of a typical vault is depicted in Figure 3-4.

The 244-AR Vault, designed and constructed between 1964 and 1968, was intended for use as a lag storage and transfer facility for PUREX neutralized current acid waste to and from B Plant and the AY and AZ Tank Farms (WHC, 1993). Beginning in 1973, PUREX sludge was sluiced from tanks in the $A$ and $A X$ Tank Farms into the $A R$ Vault, where most solids were removed from the sluicing solution by the either the addition flocculents or centrifugation. Strontium was removed by solvent extraction and the waste was returned to the waste storage tanks (Jungfleisch, 1984). The vault's canyon building measures $28.7 \mathrm{~m}(94 \mathrm{ft}$ ) by $5.49 \mathrm{~m}$ (18 ft) and contains three process cells. Four tanks are located in the cells: Tanks 001 and 002 have capacities of $189,000 \mathrm{~L}(50,000 \mathrm{gal})$, and Tanks 003 and 004 have capacities of approximately $18,900 \mathrm{~L}(5,000 \mathrm{gal})(\mathrm{WHC}, 1993)$.

The 244-BXR Vault, located south of the BX Tank Farm, was constructed between 1950 and 1951 to aid in the recovery of uranium from bismuth phosphate metal wastes. The vault began operating in 1952 and became inactive in 1956. The waste in the vault was difficult to handle, so the vault was high-pressure steam jetted in 1976. The vault was interim stabilized in 1985 (Brevick et al., 1994a). 
Figure 3-4. Configuration of a Typical Vault.

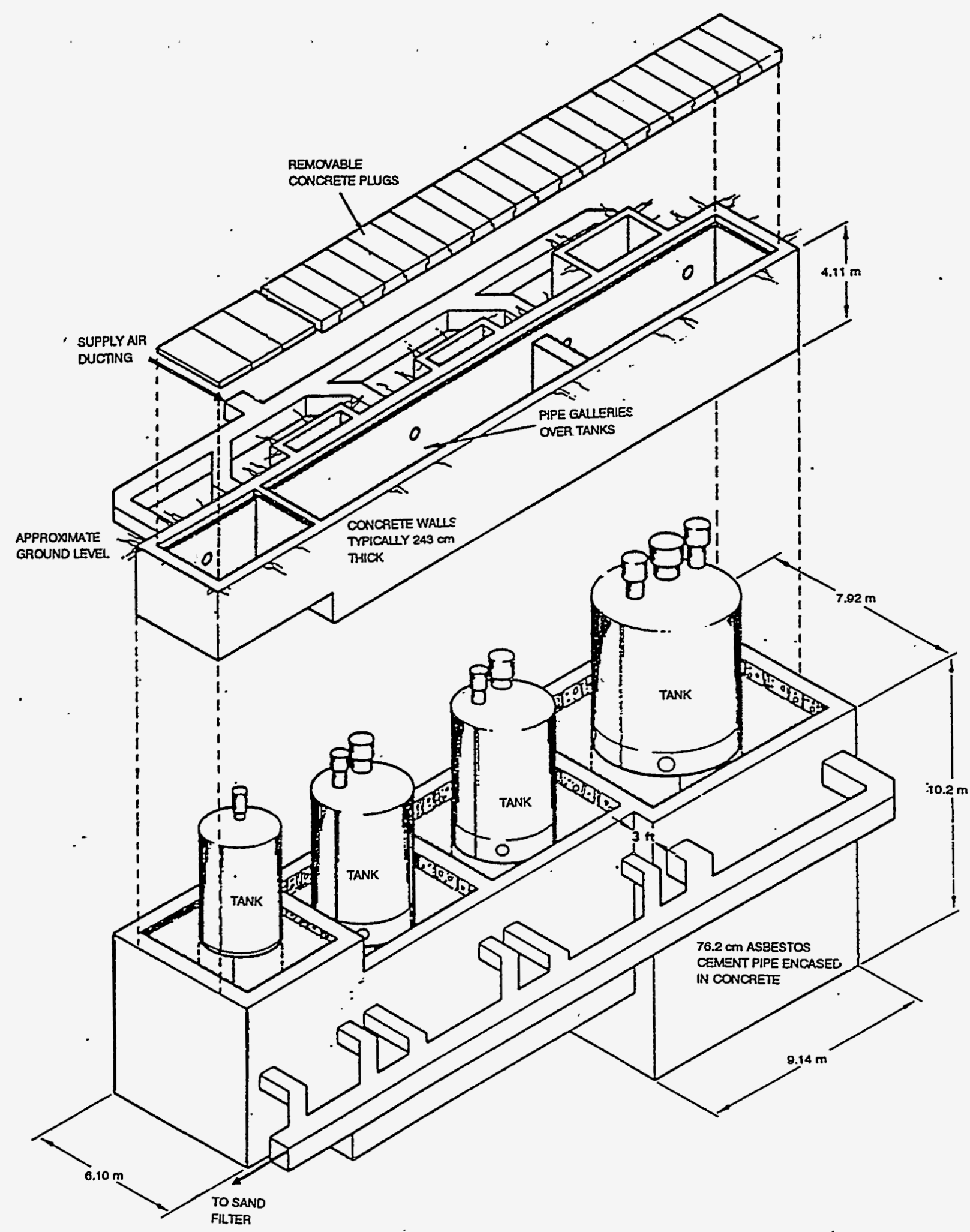


The 244-CR Vault was constructed in 1952 to aid in the recovery of uranium from bismuth phosphate metal waste. The CR Vault also was used for in-farm ferrocyanide scavenging from 1955-57 (see Section 3.2.3 for a further discussion of ferrocyanide scavenging). In addition, the vault received waste from the Strontium Semiworks en route to the $C$ Tank Farm. The CR Vault houses two 151,000-L (40,000-gal) tanks (CR-011 and CR-001) and two 56,800-L (15,000-gal) tanks (CR-002 and CR-003). Tank CR-003 is used for the interim storage of salt well waste pumped from tanks in the C Tank Farm. The salt well waste is collected from the C Tank Farm and then transferred from the CR Vault to a designated double-shell tank (WHC, 1993).

The 244-UR Vault, located directly north of the U Tank Farm, and the 244-TXR Process Vault, located just south of the TX Tank Farm, were constructed to aid in the recovery of uranium from bismuth phosphate metal wastes. The 241-WR Process Vault, also known as the 241-WR Diversion Station, consists of a large concrete underground structure containing nine process cells; each cell contains a $189,000-\mathrm{L}(50,000-\mathrm{gal})$ tank. The overall dimensions of the vault are

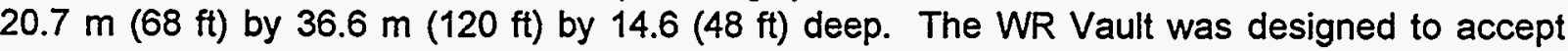
bismuth phosphate process waste through various tank farms. The vault also received waste from the other uranium recovery vaults (Brevick et al., 1994b).

Miscellaneous Sources and Wastes. There are various other sources of waste, as well as other material added to the tanks (Jungfleisch, 1984). Some wastes are from the 300 Area, 100 Area production reactors, various laboratories, and catch tanks. Unique additions to the wastes included laboratory wastes, shroud tubes, ceramic balls, experimental fuel elements, and relatively small amounts of enriched uranium, plutonium, cobalt, and natural uranium. Also, diatomaceous earth was added to Tanks BX-102, SX-113, TY-106, TX-116, TX-117, and U-104 to absorb residual supernatant liquors. For the same reason, type 2 Portland cement was added to Tank BX-105 in 1966. Miscellaneous waste streams include the following: diatomaceous earth (DE), Hanford defense residual liquor (HDRL), Hanford laboratory operations (HLO), filtered Hanford Site water $\left(\mathrm{H}_{2} \mathrm{O}\right)$, phosphate decontamination waste from $\mathrm{N}$ reactor $(\mathrm{N})$, and noncomplexed waste (NCPL)(Brevick et al., 1994a).

\subsubsection{Current Waste Sources}

- The double-shell tanks continue to receive waste generated by decommissioning and cleanup operations in the $100,200,300$, and 400 Areas. Average monthly waste generation figures for Hanford facilities are presented in Table 3-3. Waste streams that continue to be directed to the double-shell tanks are identified as follows: effluents associated with implementing the deactivation program for PUREX as well as routine maintenance and operations, waste from B Plant maintenance activities and condensate, T Plant equipment and tools decontamination and decommissioning waste, laboratory wastes from the 222-S Laboratory in the 200 West Area and the 300 Area laboratories, miscellaneous waste streams from ion exchange.resin regeneration, and equipment flush water. 
Table 3-3. Average Monthly Waste Generation. July 1, 1991, through March 31, 1994.

\begin{tabular}{|c|c|}
\hline fracilty or operation & Average Montily waste volume \\
\hline B Plant & $34,400 \mathrm{~L} \quad(9,090 \mathrm{gal})$ \\
\hline PUREX Plant & $25,400 \mathrm{~L} \quad(6,700 \mathrm{gal})$ \\
\hline T Plant & $17,300 \mathrm{~L} \quad(4,580 \mathrm{gal})$ \\
\hline Tank Farms & $14,100 \mathrm{~L} \quad(3,730 \mathrm{gal})$ \\
\hline 300 Area & $10,400 \mathrm{~L} \quad(2,760 \mathrm{gal})$ \\
\hline Redox Plant & $6,780 \mathrm{~L} \quad(1,790 \mathrm{gal})$ \\
\hline Total: & $108,000 \mathrm{~L} \quad(28,650 \mathrm{gal})$ \\
\hline
\end{tabular}

Source: (Hanton, 1994)

\subsection{WASTE MANAGEMENT OPERATIONS}

Waste management operations over the years have created a complex intermingling of the tank wastes. Nonradioactive chemicals have been added to the tanks, while varying amounts of waste, free liquid, and heat-producing radionuclides have been removed. In addition, natural processes have caused settling, stratification, and segregation of waste components, especially in the older tanks (Winters et al., 1990). This section describes the current program for remediation of the tanks and waste, along with other past and present waste management practices.

\subsubsection{Tank Waste Remediation System}

The mission of the Tank Waste Remediation System (TWRS) is to provide storage, retrieval, treatment, and disposal of the waste contained in the 177 Hanford Site waste tanks in a safe, cost effective, and environmentally sound manner (Boomer et al., 1992). The TWRS program includes characterizing tank wastes; resolving tank safety issues; and developing and implementing retrieval, pretreatment, and disposal methods. Separations processes are being developed to divide the waste into low-level, high-level, and transuranic fractions. The high-level and transuranic fractions are to be vitrified in the high-level waste plant; the low-level waste is to be vitrified in a low-level waste vitrification facility (Bell, 1994).

As discussed previously, the double-shell tanks continue to receive waste from decommissioning and clean-up activities. The single-shell tanks haven't received waste since 1980 , although water is added to one tank for evaporative cooling purposes. Interim stabilization and isolation efforts of the single-shell tanks (SSTs) continue (Boomer et al., 1992). Interim stabilization (IS) involves the removal of supernatant and interstitial liquid from the SSTs to minimize the spread of contamination should tanks begin to leak. When possible, liquid wastes are pumped to the double-shell tanks. Isolation of SSTs involves physical modifications to preclude the inadvertent addition of liquid to the tanks. Interim stabilization of all SSTs is scheduled to be completed by September 1996. 


\subsubsection{Evaporation and Concentration}

Evaporation is one of the principal tank space-saving processes used at the Hanford Site (see Table 3-4). A variety of the evaporative operations have been employed reducing the stored waste volume by more than $719,000 \mathrm{~kL}$ ( 190 million gal). The majority of waste streams were routed to the tank farm system in very dilute form which could be evaporated to $5 \%$ or less of the original volume. Each of the operations involved heating the waste, diverting the condensate, and transferring the concentrated waste to bottoms tanks to allow the salts within the concentrated waste to accumulate (Agnew, 1994a).

Table 3-4. Hanford Site Evaporator Processing Totals.

\begin{tabular}{||c|cc|cc||}
\hline Evaporator & Volume of Waste In & \multicolumn{3}{|c|}{ Volume of Waste Removed } \\
\hline $242-\mathrm{T}(1951-76)$ & $458,000 \mathrm{~kL}$ & $(121,000 \mathrm{kgal})$ & $177,000 \mathrm{~kL}$ & $(46,700 \mathrm{kgal})$ \\
\hline $242-\mathrm{B}(1951-55)$ & $123,000 \mathrm{~kL}$ & $(32,600 \mathrm{kgal})$ & $29,700 \mathrm{~kL}$ & $(7,850 \mathrm{kgal})$ \\
\hline $242-\mathrm{S}(1973-80)$ & $443,000 \mathrm{~kL}$ & $(117,000 \mathrm{kgal})$ & $234,000 \mathrm{~kL}$ & $(61,700 \mathrm{kgal})$ \\
\hline $242-\mathrm{A}(1976-91)$ & $266,000 \mathrm{~kL}$ & $(70,300 \mathrm{kgal})$ & $125,000 \mathrm{~kL}$ & $(33,000 \mathrm{kgal})$ \\
\hline BY-ITS (1965-74) & $227,000 \mathrm{~kL}$ & $(60,000 \mathrm{kgal})$ & $144,000 \mathrm{~kL}$ & $(38,100 \mathrm{kgal})$ \\
\hline Total: & $1,520,000 \mathrm{~kL}$ & $(401,000 \mathrm{kgal})$ & $709,000 \mathrm{~kL}$ & $(187,000 \mathrm{kgal})$ \\
\hline
\end{tabular}

Source: (Agnew, 1994a)

Self-Concentration. The bismuth phosphate wastes generated considerable heat through radioactive decay; however, these wastes were relatively dilute compared to some waste streams from later processes. Some wastes from REDOX and PUREX were so concentrated they would spontaneously boil. Wastes discharged to the S Tank Farm began to boil in the summer of 1952 (Gerber, 1992). Problems ensued that winter as cooling equipment not designed for self-boiling wastes froze and failed. Additional problems were caused by bumps, thermal releases and spontaneous mixing in the tank waste. Bumping is the phenomenon of violent boiling accompanied by evaporation rates up to 50 times as great as normal. The occurrence of bumping is believed to be due to the fission products carried by the settled sludge. The sludge formed on the bottom of the tank has a core of heat-producing fission isotopes and a poorer thermal conductivity than the supernatant. The combination of these phenomena produces localized regions of superheating, resulting in the observed behavior (Long, 1967). In April 1953, a study was performed to determine the practicability of allowing some REDOX wastes to selfconcentrate in the first two cascades of $S$ Tank Farm by discharging, rather than refluxing, vapor condensate (Anderson,1990). Concluding the technique was feasible, the method was implemented. Self-concentration (combined with process improvements) contributed to a $30 \%$ waste volume reduction in 1953 compared to the previous year. From the lessons learned in the $S$ Tank Farm, 10 tanks in the new SX Tank Farm were equipped with special equipment to accommodate self-boiling salt wastes from the REDOX Plant; the new $A, A X, A Y$, and $A Z$ Tank Farms were built to store self-boiling wastes from the PUREX Plant and $B$ Plant fractionation operations. Tanks designed to store self-boiling, or aging wastes, were equipped with airlift circulators to keep the contents well mixed, preventing the highly localized concentration of radionuclides suspected of causing bumping. Vapors from the boiling action were routed through headers to condensers which were vented to the atmosphere through filters. Condensate was either discarded to cribs or returned to the waste tanks to maintain desired liquid levels. Self- 
boiling was observed at rates of up to $37.9 \mathrm{~L} / \mathrm{min}$ (10 gal/min) in the SX Tank Farm and up to 114 $\mathrm{L} / \mathrm{min}$ (30 gal/min) in the A Tank Farm. High heat generation is considered to be the likely cause of failure of some tank liners in the $S$ and SX Tank Farms (Jungfleisch, 1984).

In-Plant Evaporators. Both the REDOX Plant and B. Plant treated some wastes through concentrators before discharge to the tank farms. The REDOX concentrator operated from 196772. The B Plant evaporator (cell 23) operated from 1967-68 during fractionation processing (Agnew, 1994a).

In-Tank Solidification. In-tank solidification (ITS) systems were operated in the BY Tank Farm in the 200 East Area to concentrate nonboiling aqueous (or supernatant) waste directly inside designated tanks. The ITS systems consisted of feed tanks, a tank with heating equipment installed, and downstream bottoms/recycle tanks. ITS-1 used a heated airlift recirculator in Tank BY-102; ITS-2 used electric immersion heaters and an airlift recirculator in Tank BY-112. The concentrated solutions from ITS tanks were batch transferred to the bottoms/recycle tanks. These tanks were at lower temperatures and connected to an active exhauster system for heat removal. The solutions were expected to cool in the bottoms/recycle tanks; salt cake solids would crystallize, and the saturated solution was batch transferred to the next bottoms/recycle tank, which was at an even lower temperature. This process was repeated through several bottoms tanks and the residual supernatant was eventually returned to the ITS feed tank for further concentration. The ITS systems were shut down in the mid-1970's (Grigsby et al., 1992).

Evaporator/Crystallizers. The ever-present shortage of tank space led to the construction of evaporative processing facilities for the tank farms. Four such evaporators were built near the tank farms and put into operation (see Table 3-4). The oldest is the 242-T Evaporator, built in the early 1950's to reclaim storage capacity in the tanks from nonboiling supernatant wastes. The 242-T facility was shut down in the summer of 1955 and restarted in 1965. It operated until 1976. Similarly, the 242-B Evaporator was built near the B Tank Farm and operated from 1951 to 1955. Both facilities were steam-heated pot evaporators operated at atmospheric pressure. The wastes, primarily $1 \mathrm{C}$ and UR supernatants, were partially boiled down and returned to the tank farms.

The 242-S Evaporator/Crystallizer operated from 1973-81. It was designed as a reduced pressure evaporator/crystallizer. "Evaporation was carried out until a slurry containing about 30 weight percent solids was formed. The slurry was returned to the $S$ and $S X$ Tank Farms for cooling, crystallization and settling. The 242-A Evaporator/Crystallizer began operation in 1977 and continues to be used. Modifications were made to the facility in 1987 to extend its designed operating life of 10 years. Regulatory issues led to a temporary shutdown in 1989; the facility resumed operations in 1994 following further modifications.

Evaporator waste streams 'are identified as follows: evaporator bottoms from B Plant lowlevel waste feed (BLEB); noncomplexed waste concentrated until the solution was nearly saturated with sodium aluminate, known as double-shell slurry feed (DSSF); slurry product from evaporator bottoms (EB); evaporator feed (EF); terminal liquor (TL) or double-shell slurry (DSS); Hanford defense residual liquor (HDRL); partial neutralized feed waste (PNF) (Brevick et al., 1994a; Jungfleisch, 1984). 


\subsubsection{Ferrocyanide Scavenging}

Ferrocyanide waste scavenging processes were developed at the Hanford Site in the 1950 's to provide additional storage space without constructing additional waste storage tanks. The objective was to remove the soluble, long-lived fission product, ${ }^{137} \mathrm{CS}$, from liquid wastes. This enabled the disposal of the supernate to the ground, reducing the volume of stored tank waste (Grigsby et al., 1992). Overall, 41 million gallons of tank space was recovered by scavenging (Anderson, 1990).

Three scavenging campaigns were conducted to treat three types of wastes: $U$ Plant uranium recovery waste, $T$ Plant first-cycle decontamination waste, and in-farm wastes-wastes already stored in the tank farms (Borsheim and Simpson, 1991).

In general, aqueous waste was pumped to large process tanks (approximately 189,000 $L[50,000$ gall]) where the precipitating agents-potassium ferrocyanide, sodium ferrocyanide, and nickel ions-were added. The treated waste was stirred to ensure thorough mixing and then pumped to receiver tanks in the tank farms. The precipitate was allowed to settle for a minimum of seven days; the liquid was then sampled. If the scavenging was determined to be successful, the supernate was discharged to cribs. When layers of the ferrocyanide sludge built up in the receiver tanks, they were sometimes pumped to other tanks for storage. About 140 metric tons of ferrocyanides were added to the single-shell tanks in implementing the process. Tanks that received $U$ Plant and $T$ Plant ferrocyanide sludge are estimated to contain about $80 \%$ of the ferrocyanide introduced in the scavenging process (Grigsby et al., 1992).

Scavenging of $U$ Plant waste began with a test in late 1953 during which the treated waste was routed to Tank 241-T-101. Production-scale scavenging began in September 1954. Scavenged uranium recovery waste was routed to a settling tank in the BY Tank Farm in the 200 East Area (usually Tanks $241-B Y-106,-B Y-107,-B Y-108$, or $-B Y-110$ ) at a rate of up to 189,000 $\checkmark$ day $(50,000 \mathrm{gal} /$ day). The accumulated sludge in the settling tanks was periodically transferred to Tank BY-104 or BY-105. The last scavenged waste from U Plant was received in the BY Tank Farm in June 1957 (Grigsby et al., 1992).

Routine scavenging of T Plant first-cycle decontamination (1C) wastes was started in December 1954 and continued until the plant was shut down in March 1956. Treated $1 \mathrm{C}$ waste was routed to Tanks 241-TY-101, -TY-103, and -TY-104. Six batches totaling 11,090 kL (2.93 million gal) of scavenged supernate, were disposed to the TY cribs (Grigsby et al., 1992).

Beginning in May 1955, unscavenged uranium recovery waste already stored in 200 East Area tanks was routed to the 244-CR Vault for in-farm scavenging. The scavenged waste was routed to waste storage tanks for settling, sampling, and decanting to cribs. The primary settling tanks for in-farm scavenged waste were Tanks $241-\mathrm{C}-108,-\mathrm{C}-109,-\mathrm{C}-111$, and $-\mathrm{C}-112$. In-farm scavenging was completed in December 1957. In 1956, processing was held up when it was discovered that the scavenged supernate contained unacceptable levels of ${ }^{60} \mathrm{Co}$. After considerable effort was expended in trying to develop a cobalt scavenging procedure, a decision was made to dispose of the high ${ }^{60} \mathrm{Co}$ waste to specially designated cribs and trenches. Plans were also developed for in-farm scavenging of wastes stored in the 200 West Area tanks; however, no in-tank scavenging was performed there (Grigsby et al., 1992). 


\subsubsection{Hanford Grout Disposal Program}

Grout treatment was originally planned as one of the processes for the environmental remediation of the double-shell waste tanks. It involved mixing selected low-level, double-shell tank waste (inorganic salt/water mixtures) with grout forming solids. The resulting grout slumy was to be pumped to near-surface concrete vaults for solidification and permanent disposal. However, in 1993 the DOE canceled the Grout Program following a controversial debate over the leaching of nitrates and nitrites from the grout product and the presence of high levels of ${ }^{137} \mathrm{Cs}$ in the tank waste. 
WHC-SD-WM-TI-648 REV 0

\subsection{TANK FARM OVERVIEW}

The Hanford Site currently has 177 underground storage tanks grouped in 18 tank farms (Husa et al., 1993). These radioactive waste storage tanks have two basic configurations, singleshell and double-shell. Locations of the Hanford Site tank farms are depicted in Figure 4-1.

\subsection{SINGLE-SHELL TANK BACKGROUND}

Much of the information provided in this section describing single-shell tanks was obtained from Smith (1991), Winters et al. (1990), Bell (1993), and Brevick et al. (1994a and 1994b). Information presented in the tables was acquired from Hanlon (1994), Husa et al. (1993), and Alstad (1992). Figures 4-2 through 4-5 were generated from information obtained from Hanlon (1994), Winters et al. (1990), Anderson (1990), Brevick et al. (1994a and 1994b), and Alstad (1992).

Between 1943 and 1964, 149 single-shell tanks (SSTs) were constructed in the 200 Area to store radioactive waste generated at the Hanford Site. The substantial majority of this waste was produced by irradiated uranium fuel reprocessing. Treatment facilities performed additional processing to the waste to settle, evaporate, neutralize, and condition plant wastes before and after transfer to the SSTs. Transfer facilities routed wastes between the processing plants, treatment facilities, and the SSTs.

One hundred thirty-three of the SSTs are classified as 100 series, measuring $22.9 \mathrm{~m}$ (75 $\mathrm{ft}$ ) in diameter with capacities ranging from 2,010 to $3,790 \mathrm{~kL}$ (530 to $1,000 \mathrm{kgal}$ ). The remaining 16 tanks (200 series) are much smaller with a $6.10 \mathrm{~m}$ (20 ft) diameter and a capacity of $208 \mathrm{~kL}$ (55 kgal). The SSTs are constructed of a concrete shell with steel plates lining the bottom and sides. The bottoms of most tanks are dished slightly. The dome of each tank is penetrated by risers varying in size from 10.2 to $107 \mathrm{~cm}$ (4 to $42 \mathrm{in}$.). The risers are pipes which allow access to the tank interior for tank surveillance. Most of the large $107 \mathrm{~cm}$ (42 in.) risers are 0.305 to 1.83 $\mathrm{m}(1$ to $6 \mathrm{ft})$ underground, while the small risers extend above the ground. All of the tanks have at least $1.83 \mathrm{~m}(6 \mathrm{ft})$ of earth cover to provide radiation shielding.

- Original in-tank equipment for most SSTs consisted of profile thermocouples placed at various levels within the tanks, a liquid-level sensor, sludge-level sensors, a pump, and a ventilation system. Over the years equipment has been removed, replaced, and added to accommodate new uses. Sluicers were added to agitate solids before transfer to other tanks, and condensers were installed on some tanks to remove excess heat. Three types of liquid-level sensors are currently in use. Seventy-two SSTs are equipped with a manual tape system. The remaining tanks use automatic liquid-level sensors manufactured by the Food Instrument Corporation (FIC) gauges or ENRAF 854 Liquid Level Indicator/Transmitters. The ENRAF gauges are being installed in tanks to replace the older FIC gauges (24 tanks were scheduled to be equipped with ENRAF gauges during Fiscal Year 1994). The manual surface level readings are inputted to the Computer Automated Surveillance System (CASS) manually each day. Data from the FIC and Enraf gauges are connected to the Tank Monitoring and Control System (TMACS) and transferred to CASS.

Internal tank temperatures are measured with thermocouple trees that consist of steel pipes with four to 18 thermocouples per tree, usually starting $10.2 \mathrm{~cm}$ (4 in.) from the bottom and moving up in $61 \mathrm{~cm}(2 \mathrm{ft})$ intervals. The temperatures are recorded automatically into CASS. For 
Figure 4-1. Tank Farm Locations within the Hanford Site. (Brevick et al., 1994a)
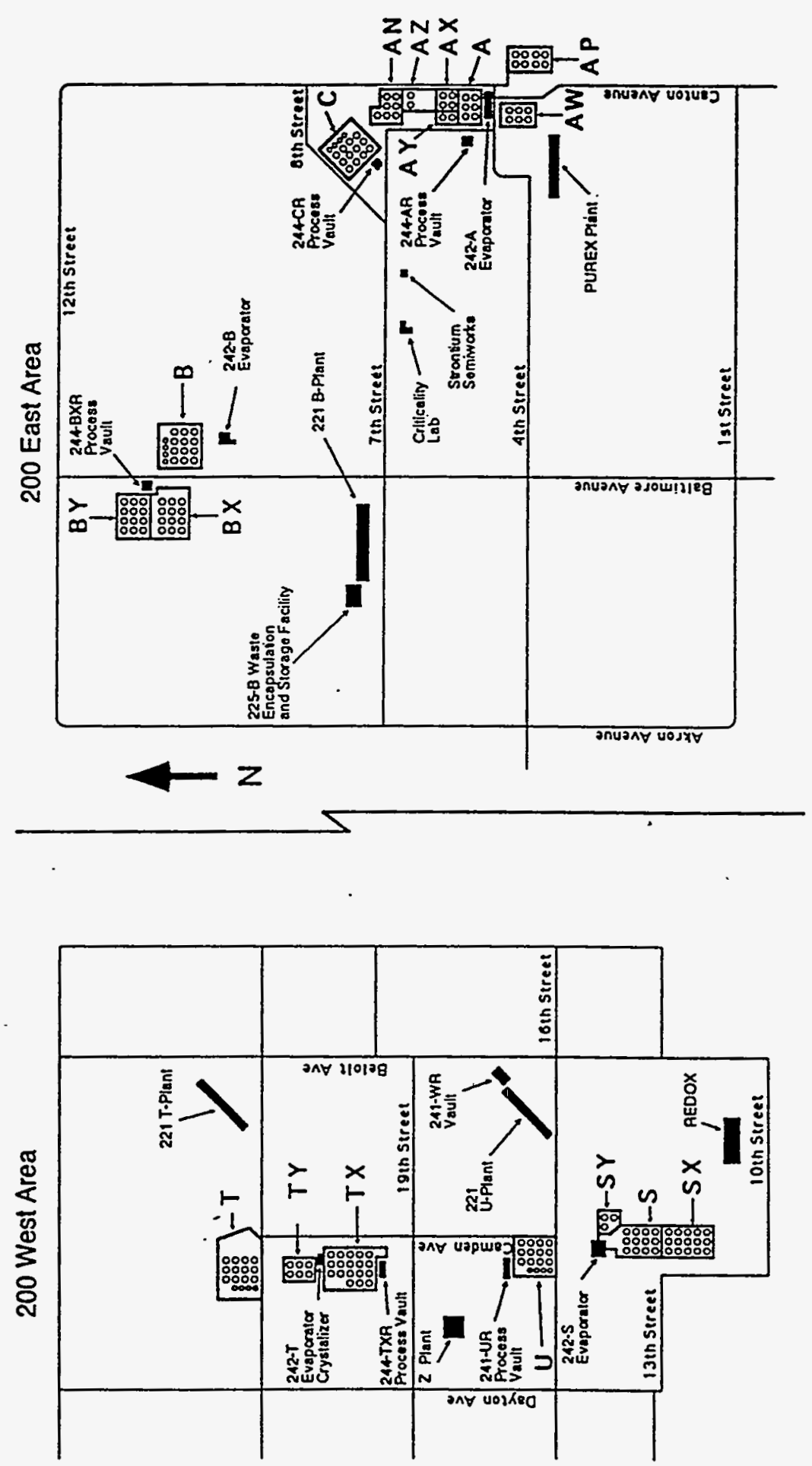
tanks that do not contain a thermocouple tree, a single thermocouple is routed down the liquid observation well to take semiannual readings.

Liquid observation wells have been installed in many tanks to determine interstitial liquid levels within the tanks. Gamma, neutron, or acoustic probes are lowered into the wells to measure the levels. The scans are fully automatic and inputted to CASS.

The anticipated design life of the SSTs was 20 to 25 years. Since the SSTs remained in active service until 1980, their service life was extended in some cases to more than 30 years, which brings into question the physical integrity of these tanks. Leak detection activities have determined 67 SSTs are either suspected or confirmed leakers.

Internal tank instrumentation and external dry wells are used to detect leaks. The drywells are located around the circumference of most tanks and throughout the tank farms to monitor for waste tank leakage. The drywells are generally $1.83 \mathrm{~m}(6 \mathrm{ft})$ in diameter and between 22.9 to $76.2 \mathrm{~m}$ (75 to $250 \mathrm{ft}$ ) deep. The term "dry" is used because they are located above the ground water table. The 241-A Tank Farm and part of the 241-SX Tank Farm also contain lateral (horizontal) drywells. Each tank has three laterals $10.2 \mathrm{~cm}$ (4 in.) in diameter and located 2.44 to $3.05 \mathrm{~m}$ ( 8 to $10 \mathrm{ft}$ ) below the concrete base of the tank. The lateral drywells are monitored by radiation detection devices and sometimes by temperature probes. Surveillance activity that detects possible radionuclide seepage prompts an investigation. Tank status is changed from "sound" to "assumed leaker" if the investigation results in a less than $95 \%$ confidence that the tank is sound. Investigations have resulted in the removal of 67 SSTs from the "sound" category. All but eight assumed leakers have been Interim Stabilized. Seven "assumed leaker" tanks remain to be stabilized and isolated. The remaining tank, 241-BY-103, is experiencing intrusion rather than leakage.

Tank stabilization and isolation programs have been put into place to prevent or minimize the waste leakage. Interim Stabilization involves the removal of supernatant and interstitial liquid from the SSTs to minimize the potential spread of contamination. These liquids are being transferred to double-shell tanks (DSTs). SSTs containing more than $189,000 \mathrm{~L}(50,000) \mathrm{gal}$ of interstitial liquid or $18,900 \mathrm{~L}(5,000 \mathrm{gal})$ of supernatant are pumped. Isolation of an SST requires physical modifications to the tank to prevent the inadvertent addition of liquid.

Two types of ventilation systems are used by the SSTs to contain airborne particulates. Active ventilation systems (exhausters) are used when the heat load is greater than $11.7 \mathrm{~kW}$ $(40,000 \mathrm{Btu} / \mathrm{h})$, or when studies predict a peak temperature greater than the operating specifications. An active ventilation system consists of: (1) a preheater to lower relative humidity in the airstream, (2) a rough filter to screen large particles, (3) Two high-efficiency particulate air (HEPA) filters, (4) a fan to draw air through the system, (5) a damper to regulate airflow, and (6) a sampler and detection unit to sample and detect radioactive particulates that are discharged. If an operating limit is exceeded, the detector deactivates the exhauster. Currently, 19 tanks are being cooled by active ventilation.

All SSTs which are not ventilated by exhausters have passive ventilation systems called breather filters. Breather filters are designed to allow passage of air at very low differential pressures to minimize pressure changes in the SSTs and prevent damage to the structures. Each breather filter assembly is composed of a single HEPA filter, pressure relief valve, and test ports. Portable exhausters are available for temporary use during planned work on passively vented tanks, and for maintenance on active ventilation systems. 


\subsubsection{1-B, C, T, and U Tank Farms}

The 241-B, C, T, and U Tank Farms were the first four tank farms, constructed in 19431944 , at the Hanford site. Each of these tank farms contain 16 tanks; 12 tanks are "100" series type and the remaining four are "200" series type. The 100 series tanks are $22.9 \mathrm{~m} \mathrm{(75} \mathrm{ft)} \mathrm{in}$ diameter with capacities of $2,010 \mathrm{~kL}(530 \mathrm{kgal}$ )(see Figure 4-2). The 200 series tanks are 6.10 $\mathrm{m}(20 \mathrm{ft})$ in diameter with a capacities of $208 \mathrm{~kL}$ (55 kgal)(see Figure 4-3). The 100 series tanks were arranged in four groups of cascading tanks with each group consisting of three tanks in a step configuration. Waste was pumped into the first, or highest tank. Waste overflow cascaded to the lower tanks through a series of transfer pipes. The cascade overflow height was approximately $4.78 \mathrm{~m}$ (188 in.) above the tank bottom at the tank wall. The 200 series tanks were used to settle waste, with the supernatant overflowing into a crib. These tanks were joined together with piping at the same elevation which allowed the waste to overflow and equalize tank volumes. All the tanks were designed for nonboiling waste with maximum temperatures of $104^{\circ} \mathrm{C}$ $\left(220^{\circ} \mathrm{F}\right)$ and $\mathrm{pH}$ of $8-10$.

\subsubsection{241-B Tank Farm}

The 241-B Tank Farm is located in the 200 East Area. The $B$ tanks were used to settle and store first- and second-cycle decontamination waste from B Plant. Tanks B-101 through B103 were sluiced for uranium recovery in 1953. Tanks 241-B-102, $-103,-106,-108,-109$, and 112 were modified to accept in-tank solidification (ITS-1 and ITS-2) evaporator bottoms. Residual liquid waste was removed from Tanks 241-B-101, -105, -107, and -110 when they were inactivated and pumped to Tank 241-B-102. The 200 series $B$ tanks received 224 (plutonium concentration facility) waste in 1952 and cascaded to a crib. Table 4-1 describes additional information pertaining to the B Tank Farm. Table 4-2 provides status of the individual tanks in the B Tank Farm, including current stabilization status as of March 1994 (Hanlon).

Table 4-1. 241-B Tank Farm Generic Description.

\begin{tabular}{|c|c|c|}
\hline Type $\quad \because \quad \ddots \quad \%$ & 100 Series Singleshell $\quad \cdots \quad \cdots$ & 200 Series Single-Shell \\
\hline Constructed & $1943-44$ & $1943-1944$ \\
\hline In Service & $1945-46$ & 1952 \\
\hline Diameter & $22.9 \mathrm{~m}(75 \mathrm{ft})$ & $6.10 \mathrm{~m}(20 \mathrm{ft})$ \\
\hline Maximum Waste Depth & $4.88 \mathrm{~m}(16 \mathrm{ft})$ & $5.70 \mathrm{~m}(18.7 \mathrm{ft})$ \\
\hline Operating Capacity & $2,010,000 \mathrm{~L}(530 \mathrm{kgal})$ & $208,000 \mathrm{~L}(55 \mathrm{kgal})$ \\
\hline Bottom Shape & Dish & Dish \\
\hline Ventilation & Passive & Passive \\
\hline $\begin{array}{l}\text { External Drywells (each } \\
\text { tank) }\end{array}$ & $\begin{array}{l}5(6:-102,4:-106,-110,3:-103,2:-104,1:-105 \\
-111) 52 \text { total }\end{array}$ & -0 \\
\hline Lateral Wells & $-0-$ & -0 \\
\hline Airlift Circulators (each) & -0 & -0 \\
\hline
\end{tabular}


Table 4-2. 241-B Tank Farm Status.

(sheet 1 of 3)

\begin{tabular}{|c|c|c|c|c|c|c|}
\hline tanin & $2418.101 \%$ & $248 \%$ & 2468.103 & $2418-104,-1$ & $2418=105$ & $2418+960$. \\
\hline Out of Service & 1974 & 1978 & 1977 & 1972 & 1977 & 1977 \\
\hline $\begin{array}{l}\text { Hanford } \\
\text { Coordinates }\end{array}$ & $\begin{array}{l}45,2377^{\circ} \\
52,552 \%\end{array}$ & $\begin{array}{l}45,337^{\circ} \mathrm{N} \\
52,552^{2} \mathrm{~W}\end{array}$ & $\begin{array}{l}45,437^{\circ} \mathrm{N} \\
52,552^{\circ} \mathrm{W}\end{array}$ & $\begin{array}{l}45,237^{\circ} \mathrm{N} \\
52,652 W\end{array}$ & $\begin{array}{l}45,337^{\circ} N \\
52,652 W W\end{array}$ & $\begin{array}{l}45,437^{\circ} N \\
52,652 \% W\end{array}$ \\
\hline Risers, Total & 12 & 10 & 11 & 10 & 12 & 10 \\
\hline FIC Surface Level & Yes & Yes & Yes & No & No & Yes \\
\hline $\begin{array}{l}\text { Manual Tape } \\
\text { Surface Level }\end{array}$ & No & No & No & Yes & Yes & No \\
\hline ENRAF Gauge & No & No & No & No & No & No \\
\hline Waste Type & NCPLX & NCPLX & NCPLX & NCPLX & NCPLX & NCPLX \\
\hline Total Waste & $\begin{array}{l}428 \mathrm{~kL}(113 \\
\mathrm{kgal})\end{array}$ & $\begin{array}{l}121 \mathrm{~kL}(32 \\
\mathrm{kgal})\end{array}$ & $\begin{array}{l}223 \mathrm{~kL}(59 \\
\mathrm{kgal})\end{array}$ & $\begin{array}{l}1,400 \mathrm{~kL}(371 \\
\mathrm{kgal})\end{array}$ & $\begin{array}{l}1,160 \mathrm{~kL}(306 \\
\text { kgal) }\end{array}$ & $\begin{array}{l}443 \mathrm{~kL}(117 \\
\text { kgal) }\end{array}$ \\
\hline Supernate & -0 & $\begin{array}{l}15.1 \mathrm{~kL}(4 \\
\mathrm{kgal})\end{array}$ & -0 & $\begin{array}{l}3.79 \mathrm{~kL}(1 \\
\mathrm{kgal})\end{array}$ & -0 & $\begin{array}{l}3.79 \mathrm{~kL}(1 \\
\text { kgal) }\end{array}$ \\
\hline $\begin{array}{l}\text { Drainable Interstitial } \\
\text { Liquid }\end{array}$ & $\begin{array}{l}22.7 \mathrm{~kL}(6 \\
\mathrm{kgal})\end{array}$ & -0 & -0 & $\begin{array}{l}174 \mathrm{~kL}(46 \\
\mathrm{kgal})\end{array}$ & $\begin{array}{l}87.1 \mathrm{~kL}(23 \\
\mathrm{kgal})\end{array}$ & $\begin{array}{l}22.7 \mathrm{~kL}(6 \\
\mathrm{kgal})\end{array}$ \\
\hline Sludge & $\begin{array}{l}428 \mathrm{~kL}(113 \\
\mathrm{kgal})\end{array}$ & $\begin{array}{l}68.1 \mathrm{~kL}(18 \\
\mathrm{kgal})\end{array}$ & $\begin{array}{l}223 \mathrm{~kL}(59 \\
\mathrm{kgal})\end{array}$ & $\begin{array}{l}1,140 \mathrm{~kL}(301 \\
\text { kgal) }\end{array}$ & $\begin{array}{l}151 \mathrm{~kL}(40 \\
\mathrm{kgal})\end{array}$ & $\begin{array}{l}439 \mathrm{~kL}(116 \\
\mathrm{kgal})\end{array}$ \\
\hline Salt cake & $-0-$ & -0 & -0 & -0 & -0 & -0 \\
\hline Integrity Category & ASMD LKR & Sound & ASMD LKR & Sound & ASMD LKR & Sound \\
\hline $\begin{array}{l}\text { Leak Vol. Accum. } \\
\text { \{last update\} }\end{array}$ & $\begin{array}{l}30.3 \mathrm{~kL}(8 \\
\mathrm{kgal})\{1989\}\end{array}$ & -0 & $\begin{array}{l}30.3 \mathrm{~kL}(8 \\
\mathrm{kgal}) \\
\{1989\}\end{array}$ & $-0-$ & $\begin{array}{l}30.3 \mathrm{~kL}(8 \\
\mathrm{kgal}) \\
\{1989\}\end{array}$ & $-\infty$ \\
\hline Watch List Status & Not Listed & Not Listed & $\begin{array}{l}\text { Organic } \\
\text { Salts }\end{array}$ & Not Listed & Not Listed & Not Listed \\
\hline Temperature & $42.8^{\circ} \mathrm{C}\left(109^{\circ} \mathrm{F}\right)$ & $\begin{array}{l}17.2^{\circ} \mathrm{C} \\
\left(63^{\circ} \mathrm{F}\right)\end{array}$ & $\begin{array}{l}16.1^{\circ} \mathrm{C} \\
\left(61^{\circ} \mathrm{F}\right)\end{array}$ & $18.3^{\circ} \mathrm{C}\left(65^{\circ} \mathrm{F}\right)$ & $18.3^{\circ} \mathrm{C}\left(65^{\circ} \mathrm{F}\right)$ & $\begin{array}{l}17.2^{\circ} \mathrm{C} \\
\left(63^{\circ} \mathrm{F}\right)\end{array}$ \\
\hline Stabillsolation & IS $/ \mathrm{P}$ & ISAP & IS/PP & ISAP & ISAP & ISAP \\
\hline
\end{tabular}


Table 4-2. 241-B Tank Farm Status.

(sheet 2 of 3)

\begin{tabular}{|c|c|c|c|c|c|c|}
\hline Tank & 2478,107 & $2418 \times 108 \%$ & $2448 \times 109$ & 2108.110 & $2418 \mathrm{~g}_{1}$ & 2413142 \\
\hline Out of Service & 1969 & 1977 & 1977 & 1971 & 1976 & 1977 \\
\hline $\begin{array}{l}\text { Hanford } \\
\text { Coordinates }\end{array}$ & $\begin{array}{l}45,237^{\circ} \mathrm{N} \\
52,752^{\circ} \mathrm{W}\end{array}$ & $\begin{array}{l}45,337^{\circ} \mathrm{N} \\
52,752^{\circ} \mathrm{W}\end{array}$ & $\begin{array}{l}45,437^{\circ} \mathrm{N} \\
52,752^{\circ} \mathrm{W}\end{array}$ & $\begin{array}{l}45,237^{\circ} \mathrm{N} \\
52,852^{\circ} \mathrm{W}\end{array}$ & $\begin{array}{l}45,337^{\circ} N \\
52,852^{\circ} W\end{array}$ & $\begin{array}{l}45,437^{\circ} \mathrm{N} \\
52,852^{\circ} \mathrm{W}\end{array}$ \\
\hline Risers, Total & 12 & 10 & 11 & 10 & 12 & 10 \\
\hline FIC Surface Level & Yes & No & Yes & Yes & No & Yes \\
\hline $\begin{array}{l}\text { Manual Tape } \\
\text { Surface Level }\end{array}$ & No & Yes & No & No & Yes & No \\
\hline ENRAF Gauge & No & No & No & No & No & No \\
\hline Waste Type & NCPLX & NCPLX & NCPLX & NCPLX & NCPLX & NCPLX \\
\hline Total Waste & $\begin{array}{l}625 \mathrm{~kL}(165 \\
\mathrm{kgal})\end{array}$ & $\begin{array}{l}356 \mathrm{~kL}(94 \\
\mathrm{kgal})\end{array}$ & $\begin{array}{l}481 \mathrm{~kL}(127 \\
\mathrm{kgal})\end{array}$ & $\begin{array}{l}931 \mathrm{~kL}(246 \\
\mathrm{kgal})\end{array}$ & $\begin{array}{l}897 \mathrm{~kL}(237 \\
\mathrm{kgal})\end{array}$ & $\begin{array}{l}125 \mathrm{~kL} \mathrm{(33} \\
\mathrm{kgal})\end{array}$ \\
\hline Supernate & $\begin{array}{l}3.79 \mathrm{~kL}(1 \\
\mathrm{kgal})\end{array}$ & -0 & -0 & $\begin{array}{l}3.79 \mathrm{~kL}(1 \\
\mathrm{kgal})\end{array}$ & $\begin{array}{l}3.79 \mathrm{~kL}(1 \\
\mathrm{kgal})\end{array}$ & $\begin{array}{l}11.4 \mathrm{~kL} \mathrm{(3} \\
\mathrm{kgal})\end{array}$ \\
\hline $\begin{array}{l}\text { Drainable Interstitial } \\
\text { Liquid }\end{array}$ & $\begin{array}{l}45.4 \mathrm{~kL} \mathrm{(12} \\
\mathrm{kgal})\end{array}$ & $\begin{array}{l}15.1 \mathrm{~kL} \mathrm{(4} \\
\mathrm{kgal})\end{array}$ & $\begin{array}{l}30.3 \mathrm{~kL} \mathrm{(8} \\
\mathrm{kgal})\end{array}$ & $\begin{array}{l}83.3 \mathrm{~kL}(22 \\
\text { kgal) }\end{array}$ & $\begin{array}{l}79.5 \text { kL (21 } \\
\text { kgal) }\end{array}$ & -0 \\
\hline Sludge & $\begin{array}{l}621 \mathrm{~kL}(164 \\
\mathrm{kgal})\end{array}$ & $\begin{array}{l}356 \mathrm{~kL}(94 \\
\mathrm{kgal})\end{array}$ & $\begin{array}{l}481 \mathrm{~kL}(127 \\
\mathrm{kgal})\end{array}$ & $\begin{array}{l}927 \mathrm{~kL}(245 \\
\mathrm{kgal})\end{array}$ & $\begin{array}{l}893 \mathrm{~kL}(236 \\
\mathrm{kgal})\end{array}$ & $\begin{array}{l}114 \mathrm{~kL}(30 \\
\mathrm{kgal})\end{array}$ \\
\hline Salt cake & -0 & -0 & $-0-$ & -0 & -0 & -0 \\
\hline Integrity Category & ASMD LKR & Sound & Sound & ASMD LKR & ASMD LKR & ASMD LKR \\
\hline $\begin{array}{l}\text { Leak Vol. Accum. } \\
\text { (last update) }\end{array}$ & $\begin{array}{l}30.3 \mathrm{~kL} \mathrm{(8} \\
\mathrm{kgal})\{1986\}\end{array}$ & -0 & -0 & $\begin{array}{l}37.9 \mathrm{~kL}(10 \\
\mathrm{kgal}) \\
\{1986\}\end{array}$ & $\begin{array}{l}30.3 \mathrm{~kL}(8 \\
\text { kgal) } \\
\{1989\}\end{array}$ & $\begin{array}{l}7.57 \mathrm{~kL}(2 \\
\text { kgal) } \\
\{1989\}\end{array}$ \\
\hline Watch List Status & Not Listed & Not Listed & Organic Salts & Not Listed & Not Listed & Not Listed \\
\hline Temperature & $15.6^{\circ} \mathrm{C}\left(60^{\circ} \mathrm{F}\right)$ & $\begin{array}{l}17.8^{\circ} \mathrm{C} \\
\left(64^{\circ} \mathrm{F}\right)\end{array}$ & $\begin{array}{l}17.2^{\circ} \mathrm{C} \\
\left(63^{\circ} \mathrm{F}\right)\end{array}$ & $17.2^{\circ} \mathrm{C}\left(63^{\circ} \mathrm{F}\right)$ & $30.0^{\circ} \mathrm{C}\left(86^{\circ} \mathrm{F}\right)$ & $\begin{array}{l}17.8^{\circ} \mathrm{C} \\
\left(64^{\circ} \mathrm{F}\right)\end{array}$ \\
\hline Stabil/solation & $I S / P P$ & IS/IP & IS $/ P$ & IS/IP & IS/IP & IS/IP \\
\hline
\end{tabular}


Table 4-2. 241-B Tank Farm Status.

(sheet 3 of 3 )

\begin{tabular}{|c|c|c|c|c|}
\hline Tank & $241.8201 \%$. & 2413.202 & $2418-203 \%$ & $241,3=204$ \\
\hline Out of Service & 1971 & 1977 & 1977 & 1977 \\
\hline Risers, Total & 8 & 9 & 9. & 9 \\
\hline FIC Surface Level & No & No & No & No \\
\hline $\begin{array}{l}\text { Manual Tape } \\
\text { Surface Level }\end{array}$ & Yes & Yes & Yes & Yes \\
\hline ENRAF Gauge • & No & No & No & No \\
\hline Waste Type & NCPLX & NCPLX & NCPLX & NCPLX \\
\hline $\begin{array}{c}\text { Total Waste } \\
\text {. }\end{array}$ & $\begin{array}{l}110 \mathrm{~kL}(29 \\
\text { kgal) }\end{array}$ & $\begin{array}{l}102 \mathrm{~kL}(27 \\
\mathrm{kgal})\end{array}$ & $\begin{array}{l}193 \mathrm{~kL}(51 \\
\mathrm{kgal})\end{array}$ & $\begin{array}{l}189 \mathrm{~kL} \text { (50 } \\
\text { kgal) }\end{array}$ \\
\hline Supernate & $\begin{array}{l}3.79 \text { kL (1 } \\
\text { kgal) }\end{array}$ & -0 & $\begin{array}{l}3.79 \mathrm{~kL} \mathrm{(1} \\
\mathrm{kgal})\end{array}$ & $\begin{array}{l}3.79 \text { kL (1 } \\
\text { kgal) }\end{array}$ \\
\hline $\begin{array}{l}\text { Drainable Interstitial } \\
\text { Liquid }\end{array}$ & $\begin{array}{l}11.4 \mathrm{~kL}(3 \\
\mathrm{kgal})\end{array}$ & $\begin{array}{l}11.4 \mathrm{~kL}(3 \\
\mathrm{kgal})\end{array}$ & $\begin{array}{l}18.9 \mathrm{~kL} \mathrm{(5} \\
\mathrm{kgal})\end{array}$ & $\begin{array}{l}18.9 \mathrm{~kL}(5 \\
\mathrm{kgal})\end{array}$ \\
\hline Sludge & $\begin{array}{l}106 \mathrm{~kL}(28 \\
\mathrm{kgal})\end{array}$ & $\begin{array}{l}102 \mathrm{~kL}(27 \\
\text { kgal) }\end{array}$ & $\begin{array}{l}188 \mathrm{~kL}(50 \\
\mathrm{kgal})\end{array}$ & $\begin{array}{l}185 \mathrm{~kL}(49 \\
\mathrm{kgal})\end{array}$ \\
\hline Salt cake & -0 & -0 & -0 & -0 \\
\hline Integrity Category & ASMD LKR & Sound & ASMD LKR & ASMD LKR \\
\hline $\begin{array}{l}\text { Leak Vol. Accum. } \\
\text { \{last update\} }\end{array}$ & $\begin{array}{l}4.54 \mathrm{~kL} \mathrm{(1.2} \\
\mathrm{kgal})\{1984\}\end{array}$ & -0 & $\begin{array}{l}1.14 \mathrm{~kL}(0.3 \\
\mathrm{kgal}) \\
\{1986\}\end{array}$ & $\begin{array}{l}1.51 \mathrm{~kL}(0.4 \\
\mathrm{kgal}) \\
\{1989\}\end{array}$ \\
\hline Watch List Status & Not Listed & Not Listed & Not Listed & Not Listed \\
\hline Temperature & $15.6^{\circ} \mathrm{C}\left(60^{\circ} \mathrm{F}\right)$ & $\begin{array}{l}15.6^{\circ} \mathrm{C} \\
\left(60^{\circ} \mathrm{F}\right)\end{array}$ & $16.1^{\circ} \mathrm{C}\left(61^{\circ} \mathrm{F}\right)$ & $\begin{array}{l}16.1^{\circ} \mathrm{C} \\
\left(61^{\circ} \mathrm{F}\right)\end{array}$ \\
\hline Stabil/solation & IS/P & ISIP & IS/IP & IS/P \\
\hline
\end{tabular}


Figure 4-2. Typical Single-Shell Tank (100 Series) Configuration (241-B, - BX, -C, $-T$, and $-U$ ).

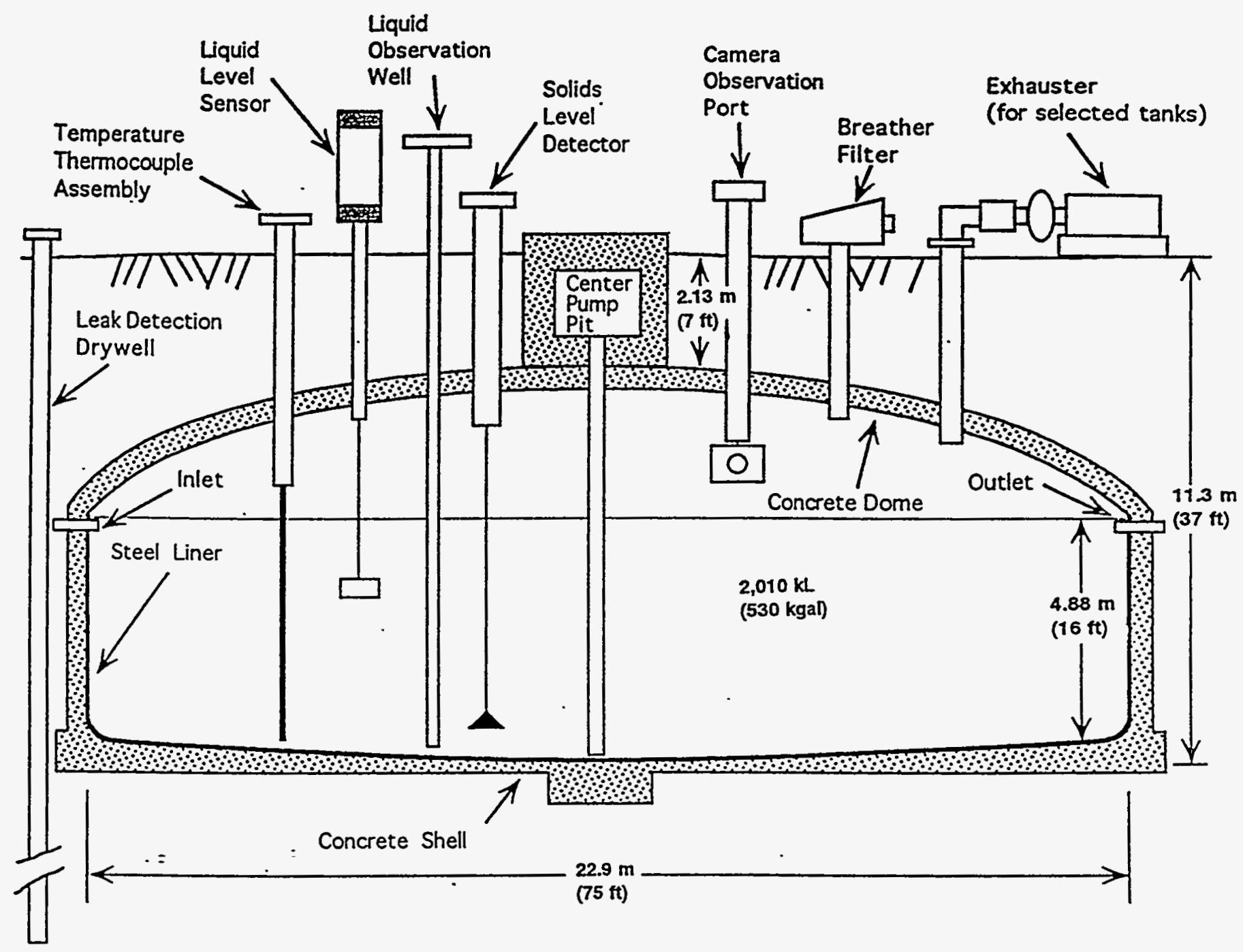


Figure 4-3. Typical Single-Shell Tank (200 Series) Configuration (241-B, $-C,-T,-U)$.

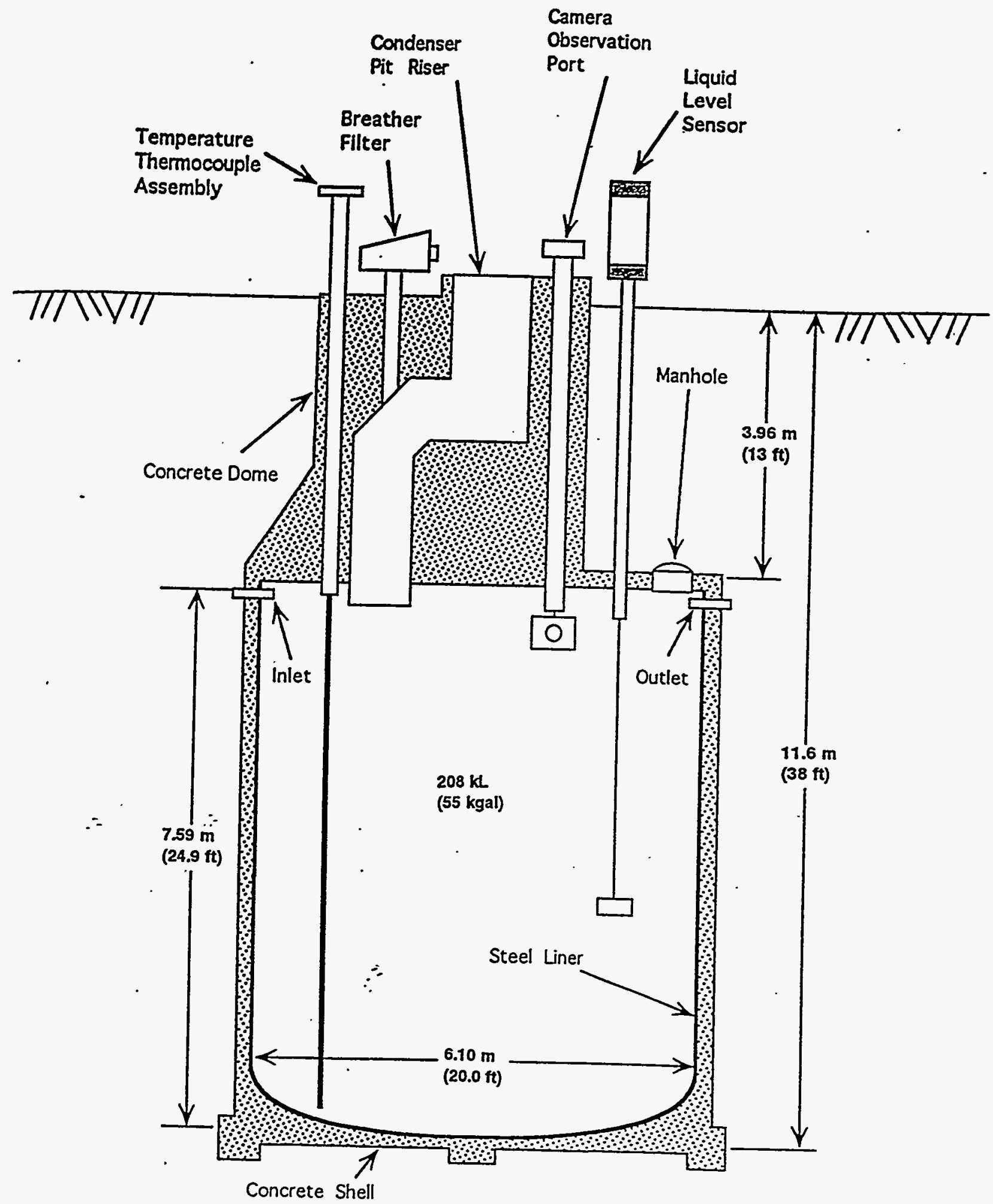




\subsubsection{241-C Tank Farm}

The 241-C Tank Farm is located in the 200 East Area. The $C$ tanks were used to support several operations. Tanks $241-C-101$ through $-C-106$ and $-C-201$ through $-C-204$ initially stored metal wastes from B Plant. Tanks $241-\mathrm{C}-107$ through $-\mathrm{C}-112$ received first-cycle decontamination waste from B Plant. Tanks 241-C-101 through $-\mathrm{C}-106$ and $-\mathrm{C}-201$ through $-\mathrm{C}-204$ were sluiced for uranium as part of the ferrocyanide scavenging program. Tanks $241-C-108,-109,-111$, and 112 used as the settling tanks. The first cascade of tanks, $C-101$ through $C-103$, were converted to TBP receiver tanks in 1953. Tank C-103 contains a floating liquid organic waste layer. The C Tank Farm also received PUREX Plant fission product (FP) waste. Tank C-106 is on the high heat load watch list due to a large amount of strontium in the waste. Periodic water additions are necessary to prevent overheating. Table 4-3 describes additional information pertaining to the C Tank Farm. Table 4-4 provides status of the individual tanks in the $C$ Tank Farm including current stabilization status as of March 1994 (Hanlon). Also, Tanks 241-C-104, -C-105 and -C 106 are ventilated actively (tank farm exhauster).

Table 4-3. 241-C Tank Farm Generic Description.

\begin{tabular}{|c|c|c|}
\hline Type & 100 seriessnglesheil, ২ & 200 sefes singles shell \\
\hline Constructed & $1943-44$ & $1943-44$ \\
\hline In Service & $1946-47$ & $1947-48$ \\
\hline Diameter & $22.9 \mathrm{~m}(75 \mathrm{ft})$ & $6.10 \mathrm{~m}(20 \mathrm{ft})$ \\
\hline Māximum Waste Depth & $4.88 \mathrm{~m}\left(16^{\circ} \mathrm{ft}\right)$ & $5.70 m(18.7 f t)$ \\
\hline Operating Capacity & $2,010,000 \mathrm{~L}(530 \mathrm{kgal})$ & 208,000 L (55 kgal) \\
\hline Bottom Shape & Dish & Dish \\
\hline Ventilation & Passive (Active: $-104,-105,-106$ ) & Passive \\
\hline $\begin{array}{l}\text { External Drywells (each } \\
\text { tank) }\end{array}$ & $\begin{array}{l}4(0:-102,3:-108,5:-103,-111,6:-106,-109,7:- \\
104,-107) 70 \text { total }\end{array}$ & -0 \\
\hline Lateral Wells & -0 & -0 \\
\hline Airlift Circulators (each) & -0 & -0 \\
\hline
\end{tabular}


Table 4-4. 241-C Tank Farm Status.

(sheet 1 of 2)

\begin{tabular}{|c|c|c|c|c|c|c|}
\hline Sonk & $241610 \%$ & $240 \%$ 102 & $2418,10 s$ & $2410 \% 10$ & $240 \% 105 \%$ & $24 \% 0106 \%$ \\
\hline Out of Service & 1970 & 1976 & 1979 & 1980 & 1979 & 1979 \\
\hline $\begin{array}{l}\text { Hanford } \\
\text { Coordinates }\end{array}$ & $\begin{array}{l}42,719^{\circ} \mathrm{W} \\
48,327^{\circ} \mathrm{W}\end{array}$ & $\begin{array}{l}42,790^{\circ} \mathrm{N} \\
48,256^{\circ} \mathrm{W}\end{array}$ & $\begin{array}{l}42,8611^{\circ} \mathrm{N} \\
48,185 \mathrm{~W}\end{array}$ & $\begin{array}{l}42,790^{\circ} \mathrm{N} \\
48,398^{\circ} \mathrm{W}\end{array}$ & $\begin{array}{l}42,861^{\circ} \mathrm{N} \\
48,327^{\circ} \mathrm{W}\end{array}$ & $\begin{array}{l}42,932^{\circ} \mathrm{N} \\
48,256^{\circ} \mathrm{W}\end{array}$ \\
\hline Risers, Total & 9 & 10 & 10 & 12 & 12 & 15 \\
\hline FIC Surface Level & No & Yes & Yes & Yes & Yes & Yes \\
\hline $\begin{array}{l}\text { Manual Tape Surface } \\
\text { Level }\end{array}$ & Yes & No & No & No & No & Yes \\
\hline ENRAF Gauge & No & No & Yes & No & No & No \\
\hline Waste Type & NCPLX & DC & NCPLX & $\mathrm{CC}$ & NCPLX & NCPLX \\
\hline Total Waste & $\begin{array}{l}333 \mathrm{~kL}(88 \\
\mathrm{kgal})\end{array}$ & $\begin{array}{l}1,600 \mathrm{~kL}(423 \\
\mathrm{kgal})\end{array}$ & $\begin{array}{l}738 \mathrm{~kL} \text { (195 } \\
\mathrm{kgal})\end{array}$ & $\begin{array}{l}1,120 \mathrm{~kL}(295 \\
\text { kgal) }\end{array}$ & $\begin{array}{l}568 \mathrm{~kL}(150 \\
\mathrm{kgal})\end{array}$ & $\begin{array}{l}867 \mathrm{~kL}(229 \\
\text { kgal) }\end{array}$ \\
\hline Supemate & -0 & -0 & $\begin{array}{l}503 \mathrm{~kL}(133 \\
\text { kgal) }\end{array}$ & -0 & -0 & $\begin{array}{l}121 \mathrm{~kL} \mathrm{(32} \\
\mathrm{kgal})\end{array}$ \\
\hline $\begin{array}{l}\text { Drainable Interstitial } \\
\text { Liquld }\end{array}$ & $\begin{array}{l}11.4 \mathrm{~kL}(3 \\
\mathrm{kgal})\end{array}$ & $\begin{array}{l}140 \mathrm{~kL}(37 \\
\mathrm{kgal})\end{array}$ & -0 & $\begin{array}{l}41.6 \mathrm{~kL}(11 \\
\mathrm{kgal})\end{array}$ & $\begin{array}{l}41.6 \mathrm{~kL}(11 \\
\mathrm{kgal})\end{array}$ & $\begin{array}{l}60.6 \mathrm{~kL}(16 \\
\mathrm{kgal})\end{array}$ \\
\hline Sludge & $\begin{array}{l}333 \mathrm{~kL}(88 \\
\mathrm{kgal})\end{array}$ & $\begin{array}{l}1,600 \mathrm{~kL}(423 \\
\mathrm{kgal})\end{array}$ & $\begin{array}{l}235 \mathrm{~kL}(62 \\
\mathrm{kgal})\end{array}$ & $\begin{array}{l}1,120 \mathrm{~kL}(295 \\
\mathrm{kgal})\end{array}$ & $\begin{array}{l}568 \mathrm{~kL}(150 \\
\mathrm{kgal})\end{array}$ & $\begin{array}{l}746 \mathrm{~kL}(197 \\
\mathrm{kgal})\end{array}$ \\
\hline Salt Cake & -0 & -0 & -0 & -0 & -0 & -0 \\
\hline Integrity Category & ASMD LKR & Sound & Sound & Sound & Sound & Sound \\
\hline $\begin{array}{l}\text { Leak Vol. Accum. } \\
\text { \{last update\} }\end{array}$ & $\begin{array}{l}75.7 \mathrm{~kL}(20 \\
\mathrm{kgal})\{1986\}\end{array}$ & -0 & -0 & -0 & -0 & -0 \\
\hline Watch List Status & Not Listed & Not Listed & Organic Salts & Not Listed & Not Listed & $\begin{array}{l}\text { High Heat } \\
\text { Load }\end{array}$ \\
\hline Temperature & $30.0^{\circ} \mathrm{C}\left(86^{\circ} \mathrm{F}\right)$ & $35.6^{\circ} \mathrm{C}\left(96^{\circ} \mathrm{F}\right)$ & $\begin{array}{l}48.9^{\circ} \mathrm{C} \\
\left(120^{\circ} \mathrm{F}\right)\end{array}$ & $29.4^{\circ} \mathrm{C}\left(85^{\circ} \mathrm{F}\right)$ & $26.7^{\circ} \mathrm{C}\left(80^{\circ} \mathrm{F}\right)$ & $\begin{array}{l}67.2^{\circ} \mathrm{C} \\
\left(153^{\circ} \mathrm{F}\right)\end{array}$ \\
\hline Stabil/isolation & ISNP & $|P|$ & $/ \mathrm{PI}$ & ISNP & IPI & IPI \\
\hline
\end{tabular}


Table 4-4. 241-C Tank Farm Status.

(sheet 2 of 2)

\begin{tabular}{|c|c|c|c|c|}
\hline rank & $24=201$ & 2416202 & $244 c-203$ & 2410204 \\
\hline Out of Service & 1977 & 1977 & 1977 & 1977 \\
\hline Risers, Total & 6 & 6 & 6 & 6 \\
\hline FIC Surface Level & No & No & No & No \\
\hline $\begin{array}{l}\text { Manual Tape Surface } \\
\text { Level }\end{array}$ & Yes & Yes & Yes & Yes \\
\hline ENRAF Gauge & No & No & No & No \\
\hline Waste Type & NCPLX & Empty & NCPLX & NCPLX \\
\hline Total Waste & $\begin{array}{l}7.57 \mathrm{~kL}(2 \\
\mathrm{kgal})\end{array}$ & $\begin{array}{l}3.79 \mathrm{~kL}(1 \\
\mathrm{kgal})\end{array}$ & $\begin{array}{l}18.9 \mathrm{~kL}(5 \\
\mathrm{kgal})\end{array}$ & $\begin{array}{l}11.4 \mathrm{~kL}(3 \\
\mathrm{kgal})\end{array}$ \\
\hline Supernate & -0 & -0 & -0 & -0 \\
\hline $\begin{array}{l}\text { Drainable Interstitial } \\
\text { Liquid }\end{array}$ & -0 & -0 & -0 & -0 \\
\hline Sludge & $\begin{array}{l}7.57 \mathrm{~kL}(2 \\
\mathrm{kgal})\end{array}$ & $\begin{array}{l}3.79 \mathrm{~kL}(1 \\
\mathrm{kgal})\end{array}$ & $\begin{array}{l}18.9 \mathrm{~kL}(5 \\
\mathrm{kgal})\end{array}$ & $\begin{array}{l}11.4 \mathrm{~kL}(3 \\
\mathrm{kgal})\end{array}$ \\
\hline Salt Cake & -0 & -0 & -0 & -0 \\
\hline Integrity Category & ASMD LKR & ASMD LKR & ASMD LKR & ASMD LKR \\
\hline $\begin{array}{l}\text { Leak Vol. Accum. } \\
\text { \{last update\} }\end{array}$ & $\begin{array}{l}2.08 \mathrm{~kL}(0.55 \\
\mathrm{kgal})\{1987\}\end{array}$ & $\begin{array}{l}1.70 \mathrm{~kL}(0.45 \\
\mathrm{kgal}) \\
\{1987\}\end{array}$ & $\begin{array}{l}1.51 \mathrm{~kL}(0.4 \\
\text { kgal) } \\
\{1986\}\end{array}$ & $\begin{array}{l}1.32 \mathrm{~kL}(0.35 \\
\mathrm{kgal}) \\
\{1987\}\end{array}$ \\
\hline Watch List Status & Not Listed & Not Listed & Not Listed & Not Listed \\
\hline Temperature & $13.3^{\circ} \mathrm{C}\left(56^{\circ} \mathrm{F}\right)$ & $15.6^{\circ} \mathrm{C}\left(60^{\circ} \mathrm{F}\right)$ & $15.0^{\circ} \mathrm{C}\left(59^{\circ} \mathrm{F}\right)$ & O/S \\
\hline Stabil/lsolation & IS/IP & IS $/ \mathrm{P}$ & ISAP & IS IP \\
\hline
\end{tabular}




\subsubsection{241-T Tank Farm}

The 241-T Tank Farm is located in the 200 West Area. Tanks 241-T-101 through -T-103 began receiving metal waste from the $\mathrm{T}$ Plant $\mathrm{BiPO}_{4}$ process in late 1945 . The other tanks received decontamination-cycle wastes. After several years, the tanks were emptied and used . for ion exchange wastes, 221-T Plant wastes, and TBP waste from the CR area (after the 241-C Tank Farm became full). Table 4-5 describes additional information pertaining to the $T$ Tank Farm. Table 4-6 provides status of the individual tanks in the T Tank Farm, including current stabilization status as of March 1994 (Hanlon).

Table 4-5. 241-T Tank Farm Generic Description.

\begin{tabular}{|c|c|c|}
\hline Type & 100 senes shgleshell, & 200 seness sngles shell \\
\hline Constructed & $1943-44$ & $1943-44$ \\
\hline In Service & $1945-46$ & 1952 \\
\hline Diameter & $22.9 \mathrm{~m}(75 \mathrm{ft})$ & $6.10 \mathrm{~m}(20 \mathrm{ft})$ \\
\hline Maximum Waste Depth & $4.88 \mathrm{~m}(16 \mathrm{ft})$ & $5.70 m(18.7 f t)$ \\
\hline Operating Capacity & $2,010,000$ L $(530 \mathrm{kgal})$ & 208,000 L (55 kgal) \\
\hline Bottom Shape & Dish & Dish \\
\hline Ventilation & Passive & Passive \\
\hline $\begin{array}{l}\text { External Drywells (each } \\
\text { tank) }\end{array}$ & $\begin{array}{l}6(3:-105,-107,-112,3:-110,5:-101,-104,-111, \\
9:-106)\end{array}$ & $-0-$ \\
\hline Lateral Wells & -0 & -0 \\
\hline Aillift Circulators: (each) & -0 & -0 \\
\hline
\end{tabular}


Table 4-6. 241-T Tank Farm Status. (sheet 1 of 3 )

\begin{tabular}{|c|c|c|c|c|c|c|}
\hline ank & $2 x_{11}=100$ & $241+102$ & 2410108 & $24810 \%$ & $28 \% 105$ & 2491806 \\
\hline Out of Service & 1979 & 1976 & 1974 & 1974 & 1976 & 1973 \\
\hline $\begin{array}{l}\text { Hanford } \\
\text { Coordinates }\end{array}$ & $\begin{array}{l}43,647^{\circ} \mathrm{W} \\
75,637^{\circ} \mathrm{W}\end{array}$ & $\begin{array}{l}43,647^{\circ} \mathrm{N} \\
75,737^{\circ} \mathrm{W}\end{array}$ & $\begin{array}{l}43,647^{\circ} \mathrm{N} \\
75,837^{\circ} \mathrm{W}\end{array}$ & $\begin{array}{l}43,547^{\circ} \mathrm{N} \\
75,637^{\circ} \mathrm{W}\end{array}$ & $\begin{array}{l}43,547^{\circ} \mathrm{N} \\
75,737^{\circ} \mathrm{W}\end{array}$ & $\begin{array}{l}43,547^{\circ} \mathrm{N} \\
75,837^{\circ} \mathrm{W}\end{array}$ \\
\hline Risers, Total & 13 & 10 & 10 & 9 & 9 & 9 \\
\hline FIC Surface Level & Yes & Yes & Yes & No & Yes & Yes \\
\hline $\begin{array}{l}\text { Manual Tape Surface } \\
\text { Level }\end{array}$ & No & No & No & Yes & No & No \\
\hline ENRAF Gauge & No & Yes & No & No & No & No \\
\hline Waste Type & NCPLX & NCPLX & NCPLX & NCPLX & NCPLX & NCPLX \\
\hline Total Waste & $\begin{array}{l}386 \mathrm{~kL}(102 \\
\text { kgal) }\end{array}$ & $\begin{array}{l}121 \mathrm{~kL}(32 \\
\text { kgai) }\end{array}$ & $\begin{array}{l}102 \mathrm{~kL}(27 \\
\mathrm{kgal})\end{array}$ & $\begin{array}{l}1,680 \mathrm{~kL}(445 \\
\mathrm{kgal})\end{array}$ & $\begin{array}{l}371 \mathrm{~kL}(98 \\
\mathrm{kgal})\end{array}$ & $\begin{array}{l}79.5 \mathrm{~kL}(21 \\
\text { kgal) }\end{array}$ \\
\hline Supernate & $\begin{array}{l}3.79 \mathrm{~kL}(1 \\
\mathrm{kgal})\end{array}$ & $\begin{array}{l}49.2 \mathrm{~kL}(13 \\
\mathrm{kgal})\end{array}$ & $\begin{array}{l}15.1 \mathrm{~kL} \mathrm{(4} \\
\mathrm{kgal})\end{array}$ & $\begin{array}{l}11.4 \mathrm{~kL} \mathrm{(3} \\
\mathrm{kgal})\end{array}$ & -0 & $\begin{array}{l}7.57 \mathrm{~kL} \mathrm{(2} \\
\mathrm{kgal})\end{array}$ \\
\hline $\begin{array}{l}\text { Drainable Interstitial } \\
\text { Liquid }\end{array}$ & $\begin{array}{l}60.6 \mathrm{~kL} \quad(16 \\
\mathrm{kgal})\end{array}$ & -0 & -0 & $\begin{array}{l}178 \mathrm{~kL} \quad(47 \\
\text { kgal) }\end{array}$ & $\begin{array}{l}87.1 \mathrm{~kL} \quad(23 \\
\mathrm{kgal})\end{array}$ & -0 \\
\hline Sludge & $\begin{array}{l}382 \mathrm{~kL}(101 \\
\mathrm{kgal})\end{array}$ & $\begin{array}{l}71.9 \mathrm{~kL} \mathrm{(19} \\
\text { kgal) }\end{array}$ & $\begin{array}{l}87.1 \mathrm{~kL}(23 \\
\mathrm{kgal})\end{array}$ & $\begin{array}{l}1,670 \mathrm{~kL}(442 \\
\mathrm{kgal})\end{array}$ & $\begin{array}{l}371 \mathrm{~kL}(98 \\
\mathrm{kgal})\end{array}$ & $\begin{array}{l}71.9 \mathrm{~kL}(19 \\
\text { kgal) }\end{array}$ \\
\hline Salt Cake & -0 & -0 & -0 & -0 & -0 & -0 \\
\hline Integrity Category & ASMD LKR & Sound & ASMD LKR & Sound & Sound & ASMD LKR \\
\hline $\begin{array}{l}\text { Leak Vol. Accum. } \\
\text { \{last update\} }\end{array}$ & $\begin{array}{l}28.4 \mathrm{~kL}(7.5 \\
\mathrm{kgal})\{1992\}\end{array}$ & -0 & $\begin{array}{l}<3.79 \mathrm{~kL} \\
(<1 \mathrm{kgal}) \\
\{1989\}\end{array}$ & -0 & -0 & $\begin{array}{l}435 \mathrm{~kL}(115 \\
\mathrm{kgal}) \\
\{1986\}\end{array}$ \\
\hline Watch List Status & Not Listed & Not Listed & Not Listed & Not Listed & Not Listed & Not Listed \\
\hline Température & $22.2^{\circ} \mathrm{C}\left(72^{\circ} \mathrm{F}\right)$ & $20.0^{\circ} \mathrm{C}\left(68^{\circ} \mathrm{F}\right)$ & $16.7^{\circ} \mathrm{C}\left(62^{\circ} \mathrm{F}\right)$ & $16.7^{\circ} \mathrm{C}\left(62^{\circ} \mathrm{F}\right)$ & o/s & $15.6^{\circ} \mathrm{C}\left(60^{\circ} \mathrm{F}\right)$ \\
\hline Stabil/solation & IS/PI & IS/IP & IS/IP & PI & IS/PP & IS $/ \mathrm{P}$ \\
\hline
\end{tabular}


Table 4-6. 241-T Tank Farm Status.

(sheet 2 of 3)

\begin{tabular}{|c|c|c|c|c|c|c|}
\hline צank & $24.110 \%$ & $241 \% 1 \% 08$ & $241+1108$ & $247+140$. & 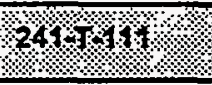 & $24+1112$ \\
\hline Out of Service & 1976 & 1974 & 1974 & 1976 & 1974 & 1977 \\
\hline $\begin{array}{l}\text { Hanford } \\
\text { Coordinates }\end{array}$ & $\begin{array}{l}43,447^{\circ} \mathrm{N} \\
75,637^{\circ} \mathrm{W}\end{array}$ & $\begin{array}{l}43,447{ }^{\circ} \mathrm{N} \\
75,737 \mathrm{~W}\end{array}$ & $\begin{array}{l}43,447^{\circ} \mathrm{N} \\
75,837^{\circ} \mathrm{W}\end{array}$ & $\begin{array}{l}43,347^{\circ} \mathrm{N} \\
75,637^{\circ} \mathrm{W}\end{array}$ & $\begin{array}{l}43,347^{\circ} N \\
75,737 \% W\end{array}$ & $\begin{array}{l}43,347^{\circ} \mathrm{N} \\
75,837^{\circ} W\end{array}$ \\
\hline Risers, Total & 9 & 9 & 9 & 9 & 9 & 9 \\
\hline FIC Surface Level & Yes & Yes & Yes & Yes & Yes & Yes \\
\hline $\begin{array}{l}\text { Manual Tape Surface } \\
\text { Level }\end{array}$ & No & Yes & No & No & No & Yes \\
\hline ENRAF Gauge & Yes & No & No & No & No & No \\
\hline Waste Type & NCPLX & NCPLX & NCPLX & NCPLX & NCPLX & NCPLX \\
\hline Total Waste & $\begin{array}{l}681 \mathrm{~kL}(180 \\
\mathrm{kgal})\end{array}$ & $\begin{array}{l}167 \mathrm{~kL}(44 \\
\mathrm{kgal})\end{array}$ & $\begin{array}{l}220 \mathrm{~kL}(58 \\
\mathrm{kgal})\end{array}$ & $\begin{array}{l}\text { 1,430 kL (379 } \\
\text { kgal) }\end{array}$ & $\begin{array}{l}1,730 \mathrm{~kL}(458 \\
\text { kgal) }\end{array}$ & $\begin{array}{l}254 \mathrm{~kL}(67 \\
\text { kgal) }\end{array}$ \\
\hline Supernate & $\begin{array}{l}34.1 \mathrm{~kL}(9 \\
\mathrm{kgal})\end{array}$ & -0 & -0 & $\begin{array}{l}11.4 \mathrm{~kL}(3 \\
\text { kgal) }\end{array}$ & $\begin{array}{l}7.57 \mathrm{~kL}(2 \\
\mathrm{kgal})\end{array}$ & $\begin{array}{l}26.5 \mathrm{~kL}(7 \\
\mathrm{kgal})\end{array}$ \\
\hline $\begin{array}{l}\text { Drainable Interstitial } \\
\text { Liquid }\end{array}$ & $\begin{array}{l}49.2 \mathrm{~kL}(13 \\
\mathrm{kgal})\end{array}$ & -0 & -0 & $\begin{array}{l}148 \mathrm{~kL}(39 \\
\mathrm{kgal})\end{array}$ & $\begin{array}{l}185 \mathrm{~kL}(49 \\
\mathrm{kgal})\end{array}$ & -0 \\
\hline Sludge & $\begin{array}{l}647 \mathrm{~kL}(171 \\
\mathrm{kgal})\end{array}$ & $\begin{array}{l}167 \mathrm{~kL}(44 \\
\mathrm{kgal})\end{array}$ & $\begin{array}{l}220 \mathrm{~kL}(58 \\
\mathrm{kgal})\end{array}$ & $\begin{array}{l}1420 \mathrm{KL}(376 \\
\mathrm{kgal})\end{array}$ & $\begin{array}{l}1,730 \mathrm{~kL}(456 \\
\text { kgal) }\end{array}$ & $\begin{array}{l}227 \mathrm{~kL}(60 \\
\mathrm{kgal})\end{array}$ \\
\hline Salt Cake & -0 & -0 & -0 & -0 & -0 & -0 \\
\hline Integrity Category & ASMD LKR & ASMD LKR & ASMD LKR & Sound & ASMD LKR & Sound \\
\hline $\begin{array}{c}\text { Leak Vol. Accum. } \\
\text { \{last update\} } \\
:\end{array}$ & $\begin{array}{l}30.3 \mathrm{~kL}(8 \\
\mathrm{kgal})\{1989\}\end{array}$ & $\begin{array}{l}<3.79 \mathrm{~kL}(<1 \\
\mathrm{kgal}) \\
\{1980\}\end{array}$ & $\begin{array}{l}<3.79 \mathrm{~kL}(< \\
1 \mathrm{kgal}) \\
\{1989\}\end{array}$ & -0 & $\begin{array}{l}<3.79 \mathrm{~kL}(<1 \\
\mathrm{kgal}) \\
\{1980\}\end{array}$ & -0 \\
\hline Watch List Status & Ferrocyanide & Not Listed & Not Listed & Hydrogen & Organic Salts & Not Listed \\
\hline Temperature & $\begin{array}{l}20.6^{\circ} \mathrm{C} \\
\left(69^{\circ} \mathrm{F}\right)\end{array}$ & $\begin{array}{l}13.9^{\circ} \mathrm{C} \\
\left(57^{\circ} \mathrm{F}\right)\end{array}$ & $\begin{array}{l}O / S \\
23.9^{\circ} \mathrm{C} \\
\left(75^{\circ} \mathrm{F} \quad 2 / 91\right.\end{array}$ & $\begin{array}{l}17.2^{\circ} \mathrm{C} \\
\left(63^{\circ} \mathrm{F}\right)\end{array}$ & $\begin{array}{l}17.2^{\circ} \mathrm{C} \\
\left(63^{\circ} \mathrm{F}\right)\end{array}$ & $\begin{array}{l}15.6^{\circ} \mathrm{C} \\
\left(60^{\circ} \mathrm{F}\right)\end{array}$ \\
\hline Stabil/solation & PI & IS $/ \mathrm{P}$ & IS/IP & PI & PI & IS/PP \\
\hline
\end{tabular}


Table 4-6. 241-T Tank Farm Status.

(sheet 3 of 3 )

\begin{tabular}{|c|c|c|c|c|}
\hline Wnok & 201201 & 2417202 & 2417203 & 241312048 \\
\hline Out of Service & 1976 & 1976 & 1976 & 1976 \\
\hline Risers, Total & 8 & 8 & 8 & 8 \\
\hline FIC Surface Level & No & No & No & No \\
\hline $\begin{array}{l}\text { Manual Tape Surface } \\
\text { Level }\end{array}$ & Yes & Yes & Yes & Yes \\
\hline ENRAF Gauge & No & No & No & No \\
\hline Waste Type & NCPLX & NCPLX & NCPLX & NCPLX \\
\hline Total Waste & $\begin{array}{l}110 \mathrm{~kL}(29 \\
\mathrm{kgal})\end{array}$ & $\begin{array}{l}79.5 \mathrm{~kL}(21 \\
\text { kgal) }\end{array}$ & $\begin{array}{l}132 \mathrm{~kL}(35 \\
\mathrm{kgal})\end{array}$ & $\begin{array}{l}144 \mathrm{~kL}(38 \\
\mathrm{kgal})\end{array}$ \\
\hline Supernate & $3.79 \mathrm{KI}(1 \mathrm{kgal})$ & -0 & -0 & -0 \\
\hline $\begin{array}{l}\text { Drainable Interstitial } \\
\text { Liquid }\end{array}$ & $\begin{array}{l}11.4 \mathrm{~kL} \mathrm{(3} \\
\mathrm{kgal})\end{array}$ & $\begin{array}{l}7.57 \mathrm{~kL} \text { (2 } \\
\mathrm{kgal})\end{array}$ & $\begin{array}{l}15.1 \mathrm{~kL}(4 \\
\mathrm{kgal})\end{array}$ & $\begin{array}{l}15.1 \mathrm{~kL}(4 \\
\text { kgal) }\end{array}$ \\
\hline Sludge & $\begin{array}{l}106 \mathrm{~kL}(28 \\
\mathrm{kgal})\end{array}$ & $\begin{array}{l}79.5 \mathrm{~kL}(21 \\
\text { kgal) }\end{array}$ & $\begin{array}{l}132 \mathrm{~kL}(35 \\
\mathrm{kgal})\end{array}$ & $\begin{array}{l}144 \mathrm{~kL}(38 \\
\text { kgal) }\end{array}$ \\
\hline Salt Cake & -0 & -0 & -0 & -0 \\
\hline Integrity Category & Sound & Sound & Sound & Sound \\
\hline $\begin{array}{l}\text { Leak Vol. Accum. } \\
\text { \{last update\} }\end{array}$ & -0 & -0 & -0 & -0 \\
\hline Watch List Status & Not Listed & Not Listed & Not Listed & Not Listed \\
\hline Temperature & $15.6^{\circ} \mathrm{C}\left(60^{\circ} \mathrm{F}\right)$ & $16.7^{\circ} \mathrm{C}\left(62^{\circ} \mathrm{F}\right)$ & $17.8^{\circ} \mathrm{C}\left(64^{\circ} \mathrm{F}\right)$ & $\begin{array}{l}17.2^{\circ} \mathrm{C} \\
\left(63^{\circ} \mathrm{F}\right)\end{array}$ \\
\hline Stabil/lsolation & IS $/ \mathrm{P}$ & IS $/ \mathrm{P}$ & ISAP & IS/IP \\
\hline
\end{tabular}




\subsubsection{4 $241-U$ Tank Farm}

The 241-U Tank Farm is located in the 200 West Area. Tanks 241-U-101 through -U-109 received metal waste from $T$ Plant and were subsequently sluiced back to the TBP recovery process in U Plant and scavenged to allow cribbing of supernatant liquids. In the early 1950's the 242-T Evaporator was used to concentrate nonboiling liquids in the first nine tanks. Tanks 241-U-110 through $-U-112$ received first-cycle decontamination waste which self-concentrated. These tanks were then used for REDOX waste storage and evaporator feed. In the 1970's many of the tanks received slurry from the 242-S Evaporator. Tank U-101 received a variety of solid waste items from May 1969 to June 1971 which included experimental fuel elements, shroud tubes, and samarium balls (WHC, 1993). The 200 series tanks received cladding waste and REDOX waste. Table 4-7 describes additional information pertaining to the $U$ Tank Farm. Table 4-8 provides the status of the individual tanks in the $U$ Tank Farm, including current stabilization status as of March 1994 (Hanlon).

Table 4-7. 241-U Tank Farm Generic Description.

\begin{tabular}{|c|c|c|}
\hline 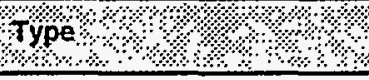 & ioo serves spgleshep & 200 senes singlestreil \\
\hline Constructed & $1943-44$ & $1943-44$ \\
\hline In Service & $\begin{array}{l}1947(1946:-101,-102,-110,1948:-106,-107 \\
1949:-108,-109)\end{array}$ & 1956. (1954: -204) \\
\hline Diameter & $22.9 \mathrm{~m}(75 \mathrm{ft})$ & $6.10 \mathrm{~m}(20 \mathrm{ft})$ \\
\hline Maximum Waste Depth & $4.88 \mathrm{~m}(16 \mathrm{ft})$ & $5.70 m(18.7 f t)$ \\
\hline Operating Capacity & $2,010,000$ L (530.kgal) & $208,000 \mathrm{~L}(55 \mathrm{kgal})$ \\
\hline Bottom Shape & Dish & Dish \\
\hline Ventilation & Passive & Passive \\
\hline $\begin{array}{l}\text { Extemal Drywells (each } \\
\text { tank) }\end{array}$ & $\begin{array}{l}4(2:-101,5:-103,-105,-110,-111,-112,6:- \\
\text { 102) } 59 \text { total }\end{array}$ & -0 \\
\hline Lateral Wells & -0 & -0 \\
\hline Airlift Circulators (each) & -0 & -0 \\
\hline
\end{tabular}


Table 4-8. 241-U Tank Farm Status.

(sheet 1 of 3 )

\begin{tabular}{|c|c|c|c|c|c|c|}
\hline rank $\%$ א & 245010 & 3414102 & 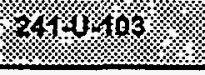 & 249104 & $2410 \% 05$ & 2439406 \\
\hline Out of Service & 1960 & 1979 & 1978 & 1951 & 1978 & 1977 \\
\hline $\begin{array}{l}\text { Hanford } \\
\text { Coordinates }\end{array}$ & $\begin{array}{l}38,197^{\circ} \mathrm{N} \\
75,637^{\circ} \mathrm{W}\end{array}$ & $\begin{array}{l}38,197^{\circ} \mathrm{N} \\
75,737 \mathrm{~W}\end{array}$ & $\begin{array}{l}38,197^{\circ} \mathrm{N} \\
75,837^{\circ} \mathrm{W}\end{array}$ & $\begin{array}{l}38,097^{\circ} \mathrm{N} \\
75,637^{\circ} W\end{array}$ & $\begin{array}{l}38,097^{\circ} \mathrm{N} \\
75,737^{\circ} \mathrm{W}\end{array}$ & $\begin{array}{l}38,097^{\circ} \mathrm{N} \\
75,837^{\circ} \mathrm{W}\end{array}$ \\
\hline Risers, Total & 10 & 13 & 12 & 10 & 12 & 13 \\
\hline FIC Surface Level & No & Yes & Yes & No & Yes & Yes \\
\hline $\begin{array}{l}\text { Manual Tape } \\
\text { Surface Level }\end{array}$ & Yes & No & No & Yes & No & No \\
\hline ENRAF Gauge & No & No & Yes & No & Yes & Yes \\
\hline Waste Type & NCPLX & NCPLX & NCPLX & NCPLX & NCPLX & NCPLX \\
\hline Total Waste & $\begin{array}{l}94.6 \mathrm{~kL}(25 \\
\mathrm{kgal})\end{array}$ & $\begin{array}{l}1,400 \mathrm{~kL}(374 \\
\mathrm{kgal})\end{array}$ & $\begin{array}{l}1,770 \mathrm{~kL}(468 \\
\text { kgal) }\end{array}$ & $\begin{array}{l}462 \mathrm{~kL}(122 \\
\mathrm{kgal})\end{array}$ & $\begin{array}{l}1,580 \mathrm{~kL}(418 \\
\mathrm{kgal})\end{array}$ & $\begin{array}{l}855 \mathrm{~kL}(226 \\
\mathrm{kgal})\end{array}$ \\
\hline Supemate & $\begin{array}{l}11.4 \mathrm{~kL}(3 \\
\mathrm{kgal})\end{array}$ & $\begin{array}{l}68.1 \mathrm{~kL}(18 \\
\mathrm{kgal})\end{array}$ & $\begin{array}{l}49.2 \mathrm{~kL}(13 \\
\mathrm{kgal})\end{array}$ & -0 & $\begin{array}{l}140 \mathrm{~kL}(37 \\
\mathrm{kgal})\end{array}$ & $\begin{array}{l}56.8 \mathrm{~kL} \text { (15 } \\
\text { kgal) }\end{array}$ \\
\hline $\begin{array}{l}\text { Drainable Interstitial } \\
\text { Liquid }\end{array}$ & -0 & $\begin{array}{l}477 \mathrm{~kL}(126 \\
\mathrm{kgal})\end{array}$ & $\begin{array}{l}666 \mathrm{~kL}(176 \\
\text { kgal) }\end{array}$ & $\begin{array}{l}26.5 \mathrm{~kL} \mathrm{(7} \\
\text { kgal) }\end{array}$ & $\begin{array}{l}538 \text { kL (142 } \\
\text { kgal) }\end{array}$ & $\begin{array}{l}257 \mathrm{~kL}(68 \\
\mathrm{kgal})\end{array}$ \\
\hline Sludge & $\begin{array}{l}83.3 \mathrm{~kL} \mathrm{(22} \\
\mathrm{kgal})\end{array}$ & $\begin{array}{l}163 \mathrm{~kL} \mathrm{(43} \\
\mathrm{kgal})\end{array}$ & $\begin{array}{l}121 \mathrm{~kL} \mathrm{(32} \\
\mathrm{kgal})\end{array}$ & $\begin{array}{l}462 \mathrm{~kL}(122 \\
\mathrm{kgal})\end{array}$ & $\begin{array}{l}121 \mathrm{~kL} \mathrm{(32} \\
\mathrm{kgal})\end{array}$ & $\begin{array}{l}98.4 \mathrm{~kL}(26 \\
\mathrm{kgal})\end{array}$ \\
\hline Salt Cake & -0 & $\begin{array}{l}1,180 \mathrm{~kL} \mathrm{(313} \\
\text { kgal) }\end{array}$ & $\begin{array}{l}1,600 \mathrm{~kL}(423 \\
\mathrm{kgal})\end{array}$ & -0 & $\begin{array}{l}1,320 \mathrm{~kL} \mathrm{(349} \\
\text { kgal) }\end{array}$ & $\begin{array}{l}700 \mathrm{~kL}(185 \\
\text { kgal) }\end{array}$ \\
\hline Integrity Category & ASMD LKR & Sound & Sound & ASMD LKR & Sound & Sound \\
\hline $\begin{array}{l}\text { Leak Vol. Accum. } \\
\text { \{last up̃date\} }\end{array}$ & $\begin{array}{c}114 \mathrm{~kL}(30 \\
\cdot \mathrm{kgal})\{1986\}\end{array}$ & -0 & -0 & $\begin{array}{l}208 \text { kL (55 } \\
\text { kgal) } \\
\{1986\}\end{array}$ & -0 & -0 \\
\hline Watch List Status & Not Listed & Not Listed & Hydrogen & Not Listed & Hydrogen & Organic \\
\hline Temperature & $19.4^{\circ} \mathrm{C}\left(67^{\circ} \mathrm{F}\right)$ & $29.4^{\circ} \mathrm{C}\left(85^{\circ} \mathrm{F}\right)$ & $30.6^{\circ} \mathrm{C}\left(87^{\circ} \mathrm{F}\right)$ & O/S & $32.2^{\circ} \mathrm{C}\left(90^{\circ} \mathrm{F}\right)$ & $27.2^{\circ} \mathrm{C}\left(81^{\circ} \mathrm{F}\right)$ \\
\hline Stabil/solation & IS/PI & $|\dot{\mathrm{P}}|$ & $/ \mathrm{PI}$ & IS/IP & IPI & $\mid \mathrm{PI}$ \\
\hline
\end{tabular}


Table 4-8. 241-U Tank Farm Status.

(sheet 2 of 3 )

\begin{tabular}{|c|c|c|c|c|c|c|}
\hline 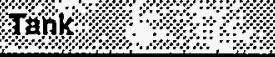 & $441 \% 107$ & 2410108 & 240409 & 2410110 & $2,17314 \%$ & 2410112 \\
\hline Out of Service & 1980 & 1979 & 1978 & 1975 & 1980 & 1970 \\
\hline $\begin{array}{l}\text { Hanford } \\
\text { Coordinates }\end{array}$ & $\begin{array}{l}37,997^{\circ} \mathrm{N} \\
75,637^{\circ} \mathrm{W}\end{array}$ & $\begin{array}{l}37,997^{\circ} \mathrm{N} \\
75,737^{\circ} \mathrm{W}\end{array}$ & $\begin{array}{l}37,997^{\circ} \mathrm{N} \\
75,837^{\circ} \mathrm{W}\end{array}$ & $\begin{array}{l}37,897^{\circ} \mathrm{N} \\
75,637^{\circ} \mathrm{W}\end{array}$ & $\begin{array}{l}37,897^{\circ} \mathrm{N} \\
75,737^{\circ} \mathrm{W}\end{array}$ & $\begin{array}{l}37,897^{\circ} \mathrm{N} \\
75,837^{\circ} \mathrm{W}\end{array}$ \\
\hline Risers, Total & 13 & 13 & 14 & 13 & 12 & 9 \\
\hline FIC Surface Level & Yes & Yes & Yes & Yes & Yes & No \\
\hline $\begin{array}{l}\text { Manual Tape } \\
\text { Surface Level }\end{array}$ & No & No & No & No & No & Yes \\
\hline ENRAF Gauge & Yes & No & Yes & No & No & No \\
\hline Waste Type & DSSF & NCPLX & NCPLX & NCPLX & DSSF & NCPLX \\
\hline Total Waste & $\begin{array}{l}1,540 \mathrm{~kL}(406 \\
\mathrm{kgal})\end{array}$ & $\begin{array}{l}1,770 \mathrm{~kL}(468 \\
\mathrm{kgal})\end{array}$ & $\begin{array}{l}1,750 \mathrm{~kL}(463 \\
\text { kgal) }\end{array}$ & $\begin{array}{l}704 \mathrm{~kL}(186 \\
\mathrm{kgal})\end{array}$ & $\begin{array}{l}1,250 \mathrm{~kL}(329 \\
\mathrm{kgal})\end{array}$ & $\begin{array}{l}185 \mathrm{~kL}(49 \\
\mathrm{kgal})\end{array}$ \\
\hline Supernate & $\begin{array}{l}117 \mathrm{~kL} \mathrm{(31} \\
\mathrm{kgal})\end{array}$ & $\begin{array}{l}90.8 \text { kL (24 } \\
\text { kgal) }\end{array}$ & $\begin{array}{l}71.9 \text { kL (19 } \\
\text { kgal) }\end{array}$ & -0 & -0 & $\begin{array}{l}15.1 \mathrm{~kL}(4 \\
\mathrm{kgal})\end{array}$ \\
\hline $\begin{array}{l}\text { Drainable Interstitial } \\
\text { Liquid }\end{array}$ & $\begin{array}{l}556 \mathrm{~kL} \text { (147 } \\
\mathrm{kgal})\end{array}$ & $\begin{array}{l}651 \text { kL (172 } \\
\text { kgal) }\end{array}$ & $\begin{array}{l}617 \text { kL (163 } \\
\text { kgal) }\end{array}$ & $\begin{array}{l}56.8 \mathrm{~kL} \mathrm{(15} \\
\text { kgal) }\end{array}$ & $\begin{array}{l}462 \mathrm{~kL}(122 \\
\text { kgal) }\end{array}$ & -0 \\
\hline Sludge & $\begin{array}{l}56.8 \mathrm{~kL} \mathrm{(15} \\
\mathrm{kgal})\end{array}$ & $\begin{array}{l}110 \mathrm{~kL}(29 \\
\mathrm{kgal})\end{array}$ & $\begin{array}{l}182 \mathrm{~kL} \mathrm{(48} \\
\mathrm{kgal})\end{array}$ & $\begin{array}{l}704 \text { kL (186 } \\
\text { kgal) }\end{array}$ & $\begin{array}{l}98.4 \text { kL (26 } \\
\text { kgal) }\end{array}$ & $\begin{array}{l}170 \mathrm{~kL}(45 \\
\mathrm{kgal})\end{array}$ \\
\hline Salt Cake & $\begin{array}{l}1,360 \mathrm{~kL}(360 \\
\text { kgal) }\end{array}$ & $\begin{array}{l}1,570 \mathrm{~kL}(415 \\
\mathrm{kgal})\end{array}$ & $\begin{array}{l}1,500 \mathrm{~kL}(396 \\
\mathrm{kgal})\end{array}$ & $-0-$ & $\begin{array}{l}1,150 \mathrm{~kL}(303 \\
\mathrm{kgal})\end{array}$ & -0 \\
\hline Integrity Category & Sound & Sound & Sound & ASMD LKR & Sound & ASMD LKR \\
\hline $\begin{array}{l}\text { Leak Vol. Accum. } \\
\text { \{last update\} }\end{array}$ & -0 . & -0 & -0 & $\begin{array}{l}18.9 \text { to } 30.7 \\
\mathrm{KL} \\
(5 \text { to } 8.1 \mathrm{kgal}) \\
\{1986\}\end{array}$ & -0 & $\begin{array}{l}32.2 \mathrm{~kL}(8.5 \\
\text { kgal) } \\
\{1986\}\end{array}$ \\
\hline Watch List Status & $\begin{array}{l}\text { Organic, } \\
\text { Hydrogen }\end{array}$ & Hydrogen & Hydrogen & Not Listed & Organic & Not Listed \\
\hline Temperature & $25.6^{\circ} \mathrm{C}\left(78^{\circ} \mathrm{F}\right)$ & $30.6^{\circ} \mathrm{C}\left(87^{\circ} \mathrm{F}\right)$ & $29.4^{\circ} \mathrm{C}\left(85^{\circ} \mathrm{F}\right)$ & $24.4^{\circ} \mathrm{C}\left(76^{\circ} \mathrm{F}\right)$ & $26.7^{\circ} \mathrm{C}\left(80^{\circ} \mathrm{F}\right)$ & $\begin{array}{l}17.2^{\circ} \mathrm{C} \\
\left(63^{\circ} \mathrm{F}\right)\end{array}$ \\
\hline Stabil/solation & /PI & /PI & IPI & IS/PI & IPI & IS $/ P$ \\
\hline
\end{tabular}


Table 4-8. 241-U Tank Farm Status.

(sheet 3 of 3 )

\begin{tabular}{|c|c|c|c|c|}
\hline Tank & $24130 \%$ & 2491202 & $241 \times 10 \%$ & 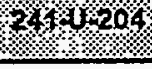 \\
\hline Out of Service & 1977 & 1977 & 1977 & 1977 \\
\hline Risers, Total & 8 & 8 & 8 & 8 \\
\hline FIC Surface Level & No & No & No & No \\
\hline $\begin{array}{l}\text { Manual Tape } \\
\text { Surface Level }\end{array}$ & Yes & Yes & Yes & Yes \\
\hline ENRAF Gauge & No & No & No & No \\
\hline Waste Type & NCPLX & NCPLX & NCPLX & NCPLX \\
\hline Total Waste & $\begin{array}{l}18.9 \mathrm{~kL}(5 \\
\mathrm{kgal})\end{array}$ & $\begin{array}{l}18.9 \mathrm{~kL}(5 \\
\mathrm{kgal})\end{array}$ & $\begin{array}{l}11.4 \mathrm{~kL} \mathrm{(3} \\
\text { kgal) }\end{array}$ & $\begin{array}{l}11.4 \mathrm{~kL} \mathrm{(3} \\
\mathrm{kgal})\end{array}$ \\
\hline Supernate & $3.79 \mathrm{KJ}(1 \mathrm{kgal})$ & $\begin{array}{l}3.79 \mathrm{KJ}(1 \\
\mathrm{kgal})\end{array}$ & $\begin{array}{l}3.79 \mathrm{Kd}(1 \\
\mathrm{kgal})\end{array}$ & $\begin{array}{l}3.79 \mathrm{KJ}(1 \\
\mathrm{kgal})\end{array}$ \\
\hline $\begin{array}{l}\text { Drainable Interstitial } \\
\text { Liquid }\end{array}$ & -0 & -0 & -0 & -0 \\
\hline Sludge & $\begin{array}{l}15.1 \mathrm{~kL}(4 \\
\mathrm{kgal})\end{array}$ & $\begin{array}{l}15.1 \mathrm{~kL}(4 \\
\mathrm{kgal})\end{array}$ & $\begin{array}{l}7.57 \mathrm{~kL}(2 \\
\mathrm{kgal})\end{array}$ & $\begin{array}{l}7.57 \mathrm{~kL}(2 \\
\mathrm{kgal})\end{array}$ \\
\hline Salt Cake & -0 & -0 & -0 & -0 \\
\hline Integrity Category & Sound & Sound & Sound & Sound \\
\hline $\begin{array}{l}\text { Leak Vol. Accum. } \\
\text { \{last update\} }\end{array}$ & $-0-$ & -0 & -0 & -0 \\
\hline Watch List Status & Not Listed & Not Listed & Not Listed & Not Listed \\
\hline Temperature & $16.1^{\circ} \mathrm{C}\left(61^{\circ} \mathrm{F}\right)$ & $16.1^{\circ} \mathrm{C}\left(61^{\circ} \mathrm{F}\right)$ & $15.6^{\circ} \mathrm{C}\left(60^{\circ} \mathrm{F}\right)$ & $\begin{array}{l}18.3^{\circ} \mathrm{C} \\
\left(65^{\circ} \mathrm{F}\right)\end{array}$ \\
\hline Stabil/solation & IS/P & IS/IP & IS/RP & IS $/ \mathrm{PP}$ \\
\hline
\end{tabular}

$\because$ 


\subsubsection{1-BX Tank Farm}

The 241-BX Tank Farm is located in the 200 East Area. This tank farm was constructed in 1948-49, using the original 241-B Tank Farm design. The tanks are arranged in four cascades of three tanks each. Tanks $24 \uparrow-B X-101$ through $-B X-106$ received $B$ Plant metal waste and $U$ Plant waste until full in 1950. These tanks were later sluiced for uranium recovery. Tanks 241BX-107 through $-B X-112$ received B Plant first-cycle waste and B Plant cell 23 concentrated waste. During the ITS-1 program, Tank 241-BX-110 received evaporator bottoms. Table 4-9 describes additional information pertaining to the BX Tank Farm. Table 4-10 provides status of the individual tanks in the BX Tank Farm, including current stabilization status as of March 1994 (Hanlon).

Table 4-9. 241-BX Tank Farm Generic Description.

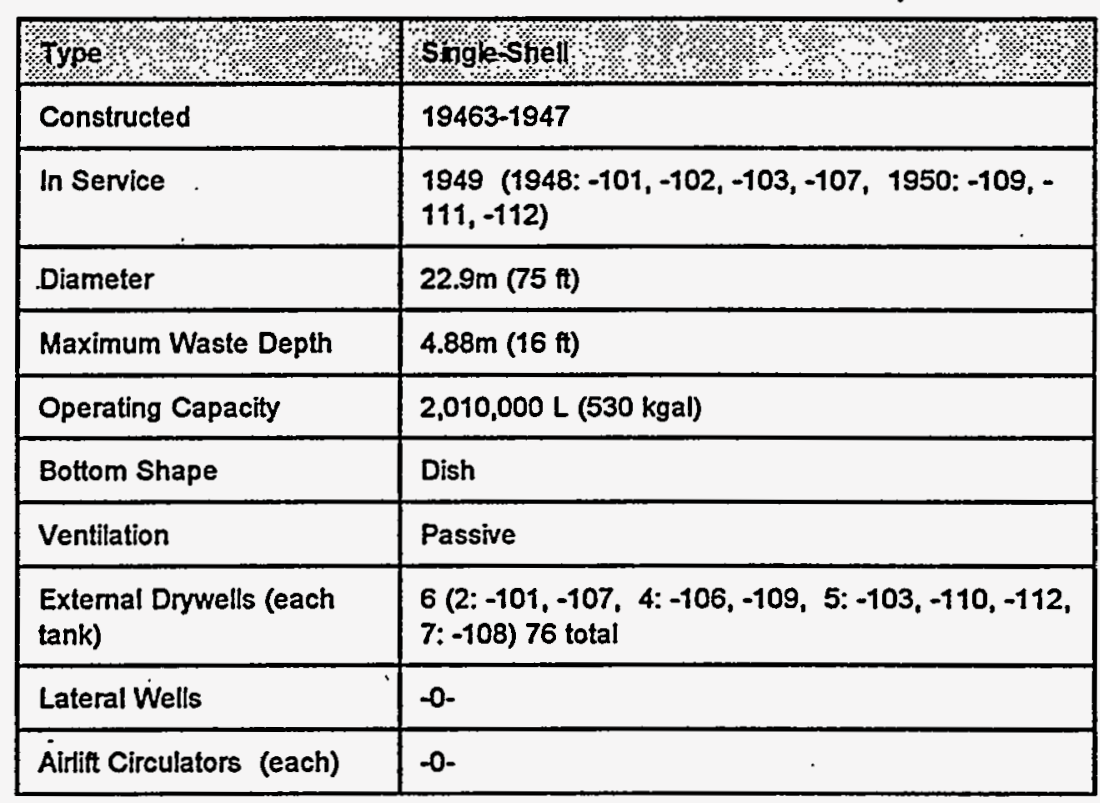


Table 4-10. 241-BX Tank Farm Status.

(sheet 1 of 2)

\begin{tabular}{|c|c|c|c|c|c|c|}
\hline Tank & $2143 x 101$ & $241 \times 3 \times 102$ & $2413 \times 103$ & $248 \mathrm{Bx} 304$ & $2418 \times 605$ & $2438 \times 106$ \\
\hline Out of Service & 1972 & 1971 & 1977 & 1980 & 1980 & 1971 \\
\hline $\begin{array}{l}\text { Hanford } \\
\text { Coordinates }\end{array}$ & $\begin{array}{l}45,400^{\circ} \mathrm{N} \\
53,250^{\circ} \mathrm{W}\end{array}$ & $\begin{array}{l}45,500^{\circ} \mathrm{N} \\
53,250^{\circ} \mathrm{W}\end{array}$ & $\begin{array}{l}45,600^{\circ} \mathrm{N} \\
53,250^{\circ} \mathrm{W}\end{array}$ & $\begin{array}{l}45,400^{\circ} \mathrm{N} \\
53,350^{\circ} \mathrm{W}\end{array}$ & $\begin{array}{l}45,500^{\circ} \mathrm{N} \\
53,350^{\circ} \mathrm{W}\end{array}$ & $\begin{array}{l}45,600 \% \mathrm{~N} \\
53,350^{\prime} \mathrm{W}\end{array}$ \\
\hline Risers, Total & 10 & 13 & 10 & 10 & 10 & 10 \\
\hline FIC Surface Level & No & No & Yes & Yes & Yes & Yes \\
\hline $\begin{array}{l}\text { Manual Tape Surface } \\
\text { Level }\end{array}$ & Yes & Yes & No & No & No & No \\
\hline ENRAF Gauge & No & No & No & No & No & Yes \\
\hline Waste Type & NCPLX & NCPLX & NCPLX & NCPLX & NCPLX & NCPLX \\
\hline Total Waste & $\begin{array}{l}163 \mathrm{~kL}(43 \\
\mathrm{kgal})\end{array}$ & $\begin{array}{l}363 \mathrm{~kL}(96 \\
\mathrm{kgal})\end{array}$ & $\begin{array}{l}250 \mathrm{~kL}(66 \\
\mathrm{kgal})\end{array}$ & $\begin{array}{l}375 \mathrm{~kL}(99 \\
\mathrm{kgal})\end{array}$ & $\begin{array}{l}193 \mathrm{~kL}(51 \\
\mathrm{kgal})\end{array}$ & $\begin{array}{l}174 \mathrm{~kL}(46 \\
\mathrm{kgal})\end{array}$ \\
\hline Supemate & $\begin{array}{l}3.79 \mathrm{~kL}(1 \\
\mathrm{kgal})\end{array}$ & -0 & $\begin{array}{l}15.1 \mathrm{~kL} \mathrm{(4} \\
\mathrm{kgal})\end{array}$ & $\begin{array}{l}11.4 \mathrm{~kL}(3 \\
\mathrm{kgal})\end{array}$ & $\begin{array}{l}18.9 \mathrm{~kL}(5 \\
\mathrm{kgal})\end{array}$ & $\begin{array}{l}56.8 \mathrm{~kL}(15 \\
\mathrm{kgal})\end{array}$ \\
\hline $\begin{array}{l}\text { Drainable Interstitial } \\
\text { Liquid }\end{array}$ & -0 . & $\begin{array}{l}15.1 \mathrm{~kL}(4 \\
\mathrm{kgal})\end{array}$ & -0 & $\begin{array}{l}114 \mathrm{~kL}(30 \\
\mathrm{kgal})\end{array}$ & $\begin{array}{l}22.7 \mathrm{~kL}(6 \\
\mathrm{kgal})\end{array}$ & -0 \\
\hline Sludge & $\begin{array}{l}159 \mathrm{~kL}(42 \\
\mathrm{kgal})\end{array}$ & $\begin{array}{l}363 \mathrm{~kL}(96 \\
\mathrm{kgal})\end{array}$ & $\begin{array}{l}235 \mathrm{~kL}(62 \\
\mathrm{kgal})\end{array}$ & $\begin{array}{l}363 \mathrm{~kL}(96 \\
\mathrm{kgal})\end{array}$ & $\begin{array}{l}163 \mathrm{~kL}(43 \\
\text { kgal) }\end{array}$ & $\begin{array}{l}117 \mathrm{~kL}(31 \\
\mathrm{kgal})\end{array}$ \\
\hline Salt Cake & -0 & -0 & $-0-$ & -0 & $\begin{array}{l}11.4 \mathrm{~kL} \mathrm{(3} \\
\mathrm{kgal})\end{array}$ & -0 \\
\hline Integrity Category & ASMD LKR & ASMD LKR & Sound & Sound & Sound & Sound \\
\hline $\begin{array}{l}\text { Leak Vol. Accum. } \\
\text { \{last update\} }\end{array}$ & $\begin{array}{l}30.3 \mathrm{~kL}(8 \\
\text { kgal) }\{1989\}\end{array}$ & $\begin{array}{l}265 \mathrm{~kL}(70 \\
\mathrm{kgal}) \\
\{1986\}\end{array}$ & -0 & -0 & -0 & - \\
\hline Watch List Status & Not Listed & Ferrocyanide & Not Listed & Not Listed & Not Listed & Ferrocyanide \\
\hline $\begin{array}{c}\text { Temperature } \\
-\end{array}$ & $\begin{array}{l}\mathrm{O} / \mathrm{S} \\
23.3^{\circ} \mathrm{C} \\
\left(74^{\circ} \mathrm{F}\right) \quad 11 / 92\end{array}$ & $\begin{array}{l}18.9^{\circ} \mathrm{C} \\
\left(66^{\circ} \mathrm{F}\right)\end{array}$ & $\begin{array}{l}\text { O/S } \\
25.0^{\circ} \mathrm{C}\left(77^{\circ} \mathrm{F}\right) \\
10 / 92\end{array}$ & $\begin{array}{l}\mathrm{O} / \mathrm{S} \\
30.6^{\circ} \mathrm{C}\left(87^{\circ} \mathrm{F}\right) \\
10 / 80\end{array}$ & $\begin{array}{l}18.9^{\circ} \mathrm{C} \\
\left(66^{\circ} \mathrm{F}\right)\end{array}$ & $\begin{array}{l}19.4^{\circ} \mathrm{C} \\
\left(67^{\circ} \mathrm{F}\right)\end{array}$ \\
\hline Stabil/solation & IS/PI & IS/P & IS/IP & IS/P & IS/IP & IPI \\
\hline
\end{tabular}


Table 4-10. 241-BX Tank Farm Status.

(sheet 2 of 2)

\begin{tabular}{|c|c|c|c|c|c|c|}
\hline Tank & $2418 \times \%$ & $24 \% 8 \times 108$ & $2418 \mathrm{BX} 109$ & $2448 \times 110$ & $2418 \times 111 \%$ & $248 \times 3 \times 12$. \\
\hline Out of Service & 1977 & 1974 & 1974 & 1977 & 1977 & 1977 \\
\hline $\begin{array}{l}\text { Hanford } \\
\text { Coordinates }\end{array}$ & $\begin{array}{l}45,400^{\circ} \mathrm{N} \\
53,450^{\circ} \mathrm{W}\end{array}$ & $\begin{array}{l}45,500^{\circ} \mathrm{N} \\
53,450^{\circ} \mathrm{W}\end{array}$ & $\begin{array}{l}45,600^{\circ} \mathrm{N} \\
53,450^{\circ} \mathrm{W}\end{array}$ & $\begin{array}{l}45,400^{\circ} \mathrm{N} \\
53,550^{\circ} \mathrm{W}\end{array}$ & $\begin{array}{l}45,500 \% \mathrm{~N} \\
53,550 \mathrm{~W}\end{array}$ & $\begin{array}{l}45,600^{\circ} \mathrm{N} \\
53,550 \mathrm{~W}\end{array}$ \\
\hline Risers, Total & 12 & 11 & 10 & 11 & 11 & $\boldsymbol{9}$ \\
\hline FIC Surface Level & Yes & No & Yes & No & No & Yes \\
\hline $\begin{array}{l}\text { Manual Tape Surface } \\
\text { Level }\end{array}$ & No & Yes & No & Yes & Yes & No \\
\hline ENRAF Gauge & No & No & No & No & No & No \\
\hline Waste Type & NCPLX & NCPLXX & NCPLX & NCPLX & NCPLX & NCPLX \\
\hline Total Waste & $\begin{array}{l}1,310 \mathrm{~kL}(345 \\
\text { kgal) }\end{array}$ & $\begin{array}{l}98.4 \mathrm{~kL}(26 \\
\mathrm{kgal})\end{array}$ & $\begin{array}{l}731 \mathrm{~kL}(193 \\
\mathrm{kgal})\end{array}$ & $\begin{array}{l}749 \mathrm{~kL} \text { (198 } \\
\mathrm{kgal})\end{array}$ & $\begin{array}{l}799 \mathrm{~kL}(211 \\
\mathrm{kgai})\end{array}$ & $\begin{array}{l}625 \mathrm{~kL}(165 \\
\mathrm{kgal})\end{array}$ \\
\hline Supernate & $\begin{array}{l}3.79 \mathrm{~kL}(1 \\
\mathrm{kgal})\end{array}$ & -0 & -0 & -0 & -0 & $\begin{array}{l}3.79 \mathrm{~kL}(1 \\
\mathrm{kgal})\end{array}$ \\
\hline $\begin{array}{l}\text { Drainable Interstitial } \\
\text { Liquid }\end{array}$ & $\begin{array}{l}110 \mathrm{~kL}(29 \\
\mathrm{kgal})\end{array}$ & $\begin{array}{l}3.79 \text { kL (1 } \\
\text { kgal) }\end{array}$ & $\begin{array}{l}49.2 \mathrm{~kL}(13 \\
\mathrm{kgal})\end{array}$ & $\begin{array}{l}56.8 \mathrm{~kL} \text { (15 } \\
\text { kgal) }\end{array}$ & -0 & $\begin{array}{l}26.5 \mathrm{~kL}(7 \\
\mathrm{kgal})\end{array}$ \\
\hline Sludge & $\begin{array}{l}1,300 \mathrm{~kL}(344 \\
\text { kgal) }\end{array}$ & $\begin{array}{l}98.4 \mathrm{~kL}(26 \\
\mathrm{kgal})\end{array}$ & $\begin{array}{l}731 \mathrm{~kL}(193 \\
\text { kgal) }\end{array}$ & $\begin{array}{l}715 \mathrm{~kL}(189 \\
\mathrm{kgal})\end{array}$ & $\begin{array}{l}257 \mathrm{~kL}(68 \\
\text { kgal) }\end{array}$ & $\begin{array}{l}621 \mathrm{~kL}(164 \\
\mathrm{kgal})\end{array}$ \\
\hline Salt Cake & -0 & $-0-$ & -0 & $\begin{array}{l}34.1 \mathrm{~kL}(9 \\
\mathrm{kgal})\end{array}$ & $\begin{array}{l}541 \mathrm{~kL}(143 \\
\mathrm{kgal})\end{array}$ & -0 \\
\hline Integrity Category & Sound & ASMD LKR & Sound & ASMD LKR & ASMD LKR & Sound \\
\hline $\begin{array}{c}\text { Leak Vol. Accum. } \\
\text { \{last update\} } \\
=\end{array}$ & $-0-$ & $\begin{array}{l}9.46 \mathrm{~kL}(2.5 \\
\mathrm{kgal}) \\
\{1986\}\end{array}$ & $-0-$ & $\begin{array}{l}30.3 \mathrm{~kL}(8 \\
\mathrm{kgal})\{1989\}\end{array}$ & $\begin{array}{l}30.3 \mathrm{~kL}(8 \\
\mathrm{kgal}) \\
\{1993\}\end{array}$ & -0 \\
\hline Watch List Status & Not Listed & Not Listed & Not Listed & Not Listed & Not Listed & Not Listed \\
\hline Temperature & $\begin{array}{l}\mathrm{O} / \mathrm{S} \\
20.6^{\circ} \mathrm{C}\left(69^{\circ} \mathrm{F}\right) \\
10 / 82\end{array}$ & $\begin{array}{l}18.3^{\circ} \mathrm{C} \\
\left(65^{\circ} \mathrm{F}\right)\end{array}$ & $\begin{array}{l}\text { O/S } \\
25.0^{\circ} \mathrm{C}\left(77^{\circ} \mathrm{F}\right) \\
7 / 93\end{array}$ & $\begin{array}{l}23.3^{\circ} \mathrm{C} \\
\left(74^{\circ} \mathrm{F}\right)\end{array}$ & $\begin{array}{l}20.6^{\circ} \mathrm{C} \\
\left(69^{\circ} \mathrm{F}\right)\end{array}$ & $\begin{array}{l}18.3^{\circ} \mathrm{C} \\
\left(65^{\circ} \mathrm{F}\right)\end{array}$ \\
\hline Stabilisolation & IS/PI & ISAP & IS/PI & IS/PI & /PI & IS/PI \\
\hline
\end{tabular}




\subsubsection{1-TX Tank Farm}

The 241-TX Tank Farm is located in the 200 West Area. The tank farm was constructed in 1948-49 using a modified 241-B Tank Farm design (see Figure 4-4). Eighteen tanks with capacities of $2,870 \mathrm{~kL}$ ( $758 \mathrm{kgal})$ each are arranged in three cascades of four tanks each, and two cascades of three tanks each. Tanks 241-TX-101 through TX-108 were filled with T Plant metal waste during the 1950's. Six of the tanks were sluiced until empty, and then received REDOX waste. The other two tanks, Tanks 241-TX-103 and TX-108, received TBP waste after sluicing. In later years these tanks were used as recycle tanks for the 242-T Evaporator and to receive evaporator bottoms. The third cascade, Tanks 241-TX-108 through TX-112, stored firstcycle decontamination waste prior to use with the 242-T Evaporator. The remaining six tanks were used in the 1950's as feed, bottoms, and recycle tanks for the 242-T Evaporator. Table 4-11 provides additional information pertaining to the TX Tank Farm. Table 4-12 provides status of the individual tanks in the TX Tank Farm, including current stabilization status as of March 1994 (Hanlon).

Table 4-11. 241-TX Tank Farm Generic Description.

\begin{tabular}{|l|l|}
\hline Type & Singlestell \\
\hline Constructed & $1947-48$ \\
\hline In Service & $\begin{array}{l}1950(1949:-101,-111,1951:-105,-106,1951:-105,-106,-114 \\
\text { through }-118)\end{array}$ \\
\hline Diameter & $22.9 \mathrm{~m}(75 \mathrm{ft})$ \\
\hline Maximum Waste Depth & $7.01 \mathrm{~m}(23 \mathrm{ft})$ \\
\hline Operating Capacity & $2,870,000 \mathrm{~L}(758 \mathrm{kgal})$ \\
\hline Bottom Shape & Dish \\
\hline Ventilation & Passive \\
\hline $\begin{array}{l}\text { External Drywells (each } \\
\text { tank) }\end{array}$ & $\begin{array}{l}6(3:-108,-113,-114,-116,4:-115,-117,5:-101,-102,-106,- \\
109,-111,7:-107,-118)\end{array}$ \\
\hline Lateral Wells & -0. \\
\hline Airlift Circulators (each) & $-0-$ \\
\hline
\end{tabular}


Figure 4-4. Typical Single-Shell Tank Configuration (241-BY, -S, -TX, and -TY).

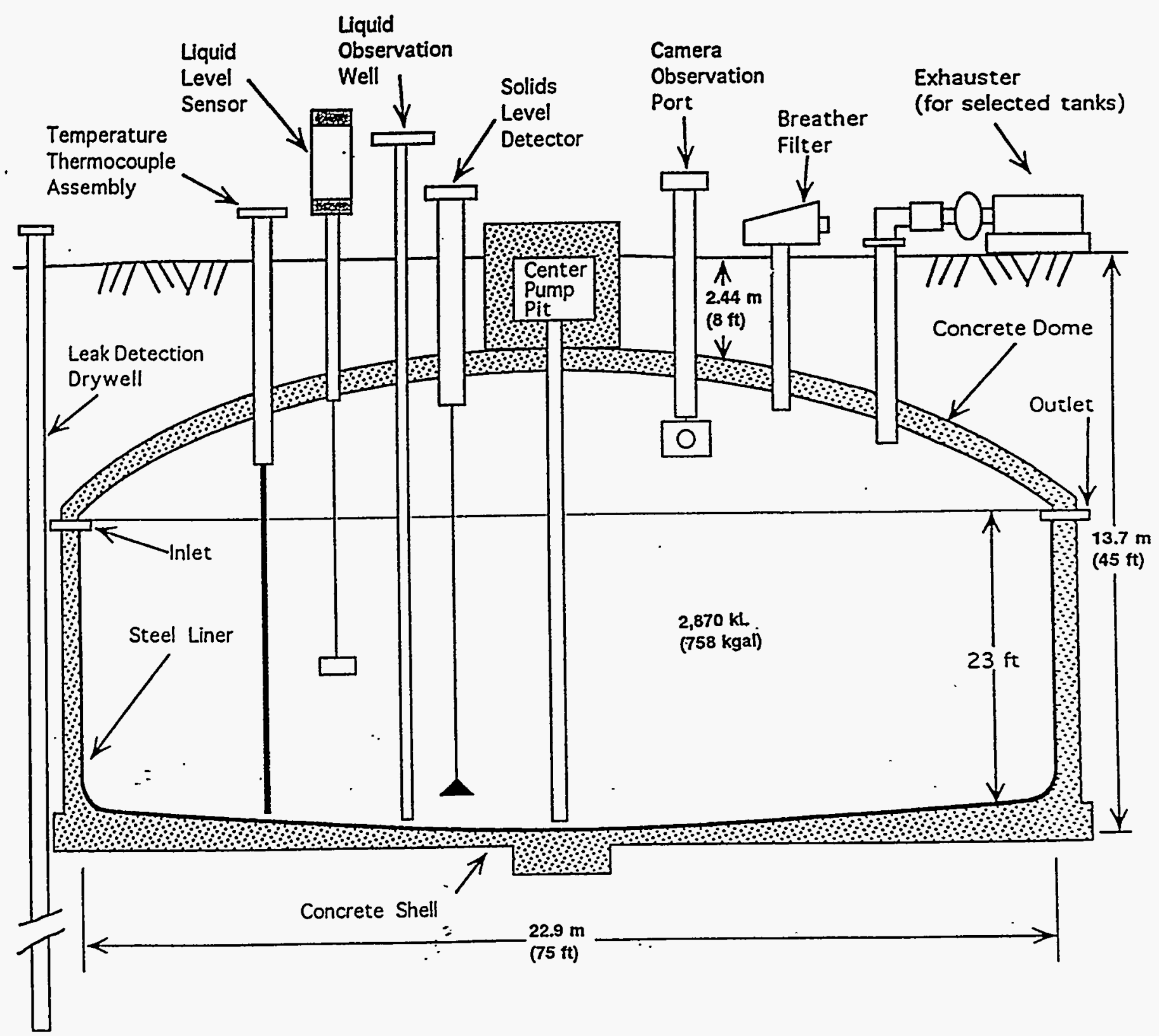


Table 4-12. 241-TX Tank Farm Status.

(sheet 1 of 3 )

\begin{tabular}{|c|c|c|c|c|c|c|}
\hline Tank $8.1 .1 .1 .4 \%$ & $241 x_{10}$ & $2417 \times 102$ & $241 \% \mathrm{x} 103$ & $2418 \times 104$ & $241,7 \times 105$ & $24811 \times 106$ \\
\hline Out of Service & 1980 & 1977 & 1980 & 1977 & 1977 & 1977 \\
\hline $\begin{array}{l}\text { Hanford } \\
\text { Coordinates }\end{array}$ & $\begin{array}{l}41,650^{\circ} \mathrm{N} \\
75,750^{\circ} \mathrm{W}\end{array}$ & $\begin{array}{l}41,650^{\circ} \mathrm{N} \\
75,852^{\circ} \mathrm{W}\end{array}$ & $\begin{array}{l}41,6500^{\circ} \mathrm{N} \\
75,954{ }^{\circ} \mathrm{W}\end{array}$ & $\begin{array}{l}41,650^{\circ} \mathrm{N} \\
76,056^{\circ} \mathrm{W}\end{array}$ & $\begin{array}{l}41,752^{\circ} \mathrm{N} \\
75,750^{\circ} \mathrm{W}\end{array}$ & $\begin{array}{l}41,752 \times N \\
75,852 \% W\end{array}$ \\
\hline Risers, Total & 16 & 23 & 16 & 16 & 16 & 19 \\
\hline FIC Surface Level & Yes & No & Yes & Yes & No & No \\
\hline $\begin{array}{l}\text { Manual Tape Surface } \\
\text { Level }\end{array}$ & No & Yes & Yes & Yes & Yes & Yes \\
\hline ENRAF Gauge & No & No & No & No & No & No \\
\hline Waste Type & NCPLX & NCPLX & NCPLX & NCPLX & NCPLX & NCPLX \\
\hline Total Waste & $\begin{array}{l}329 \mathrm{~kL}(87 \\
\mathrm{kgal})\end{array}$ & $\begin{array}{l}821 \mathrm{~kL}(217 \\
\mathrm{kgal})\end{array}$ & $\begin{array}{l}594 \mathrm{~kL}(157 \\
\mathrm{kgal})\end{array}$ & $\begin{array}{l}246 \mathrm{~kL}(65 \\
\mathrm{kgal})\end{array}$ & $\begin{array}{l}2,310 \mathrm{~kL}(609 \\
\mathrm{kgal})\end{array}$ & $\begin{array}{l}1,710 \mathrm{~kL}(453 \\
\mathrm{kgal})\end{array}$ \\
\hline Supernate & $\begin{array}{l}11.4 \mathrm{~kL}(3 \\
\mathrm{kgal})\end{array}$ & $-0-$ & -0 & $\begin{array}{l}3.79 \mathrm{~kL}(1 \\
\mathrm{kgal})\end{array}$ & -0 & $-0-$ \\
\hline $\begin{array}{l}\text { Drainable Interstitial } \\
\text { Liquid }\end{array}$ & $\begin{array}{l}7.57 \mathrm{~kL}(2 \\
\text { kgal) }\end{array}$ & $\begin{array}{l}83.3 \mathrm{~kL}(22 \\
\mathrm{kgal})\end{array}$ & $\begin{array}{l}56.8 \mathrm{~kL}(15 \\
\mathrm{kgal})\end{array}$ & $\begin{array}{l}53.0 \mathrm{~kL}(14 \\
\mathrm{kgal})\end{array}$ & $\begin{array}{l}75.7 \mathrm{~kL}(20 \\
\mathrm{kgal})\end{array}$ & $\begin{array}{l}37.9 \mathrm{~kL}(10 \\
\text { kgal) }\end{array}$ \\
\hline Sludge & $\begin{array}{l}318 \mathrm{~kL}(84 \\
\mathrm{kgal})\end{array}$ & -0 & $\begin{array}{l}594 \mathrm{~kL}(157 \\
\mathrm{kgal})\end{array}$ & $-0-$ & -0 & $-0-$ \\
\hline Salt Cake & -0 & $\begin{array}{l}821 \mathrm{~kL}(217 \\
\mathrm{kgal})\end{array}$ & -0 & $\begin{array}{l}242 \mathrm{~kL}(64 \\
\mathrm{kgal})\end{array}$ & $\begin{array}{l}2,310 \mathrm{~kL}(609 \\
\mathrm{kgal})\end{array}$ & $\begin{array}{l}1,710 \mathrm{~kL}(453 \\
\mathrm{kgal})\end{array}$ \\
\hline Integrity Category & Sound & Sound & Sound & Sound & ASMD LKR & Sound \\
\hline $\begin{array}{l}\text { Leak Vol. Accum. } \\
\text { \{last update\} } \\
=\end{array}$ & -0 & -0 & $-0-$ & -0 & $\begin{array}{l}30.3 \mathrm{~kL}(8 \\
\mathrm{kgal}) \\
\{1989\}\end{array}$ & -0 \\
\hline Watch List Status & Not Listed & Not Listed & Not Listed & Not Listed & Organic Salts & Not Listed \\
\hline Temperature & ols & ors & $21.7^{\circ} \mathrm{C}\left(71^{\circ} \mathrm{F}\right)$ & $18.3^{\circ} \mathrm{C}\left(65^{\circ} \mathrm{F}\right)$ & $\begin{array}{l}40.0^{\circ} \mathrm{C} \\
\left(104^{\circ} \mathrm{F}\right)\end{array}$ & $25.6^{\circ} \mathrm{C}\left(78^{\circ} \mathrm{F}\right)$ \\
\hline Stabil/solation & IS/IP & IS $/ \mathrm{P}$ & ISAP & IS/IP & IS/IP & IS/P \\
\hline
\end{tabular}


Table 4-12. 241-TX Tank Farm Status.

(sheet 2 of 3 )

\begin{tabular}{|c|c|c|c|c|c|c|}
\hline Tank $/ 1.1 \%$ & $241,1 \times 107$ & $244 \times 109 \%$ & $241,7-109$ & $2415 \times 110$ & $24+x+11 \%$ & $247 x=12$ \\
\hline Out of Service & 1977 & 1977 & 1977 & 1977 & 1977 & 1974 \\
\hline $\begin{array}{l}\text { Hanford } \\
\text { Coordinates }\end{array}$ & $\begin{array}{l}41,752^{\circ} \mathrm{N} \\
75,854^{\circ} \mathrm{W}\end{array}$ & $\begin{array}{l}41,752^{\circ} \mathrm{N} \\
76,056^{\circ} \mathrm{W}\end{array}$ & $\begin{array}{l}41,854^{\circ} \mathrm{N} \\
75,750^{\circ} \mathrm{W}\end{array}$ & $\begin{array}{l}41,854^{\circ} \mathrm{N} \\
75,852^{\circ} \mathrm{W}\end{array}$ & $\begin{array}{l}41,854^{\circ} \mathrm{N} \\
75,954^{\circ} \mathrm{W}\end{array}$ & $\begin{array}{l}41,854^{\circ} \mathrm{N} \\
76,056^{\circ} \mathrm{W}\end{array}$ \\
\hline Risers, Total & 16 & 17 & 15 & 20 & 21 & 16 \\
\hline FIC Surface Level & Yes & Yes & Yes & No & No & No \\
\hline $\begin{array}{l}\text { Manual Tape Surface } \\
\text { Level }\end{array}$ & Yes & Yes & Yes & Yes & Yes & Yes \\
\hline ENRAF Gauge & No & No & No & No. & No & No \\
\hline Waste Type & NCPLX & NCPLX & NCPLX & NCPLX & NCPLX & NCPLX \\
\hline Total Waste & $\begin{array}{l}136 \mathrm{~kL}(36 \\
\mathrm{kgal})\end{array}$ & $\begin{array}{l}507 \mathrm{~kL}(134 \\
\text { kgal) }\end{array}$ & $\begin{array}{l}\text { 1,450 KL (384 } \\
\text { kgal) }\end{array}$ & $\begin{array}{l}1,750 \mathrm{~kL}(462 \\
\text { kgal) }\end{array}$ & $\begin{array}{l}1,400 \mathrm{~kL}(370 \\
\text { kgal) }\end{array}$ & $\begin{array}{l}2,460 \mathrm{~kL}(649 \\
\mathrm{kgal})\end{array}$ \\
\hline Supernate & $\begin{array}{l}3.79 \mathrm{~kL}(1 \\
\text { kgal) }\end{array}$ & $-\infty$ & -0 & -0 & -0 & -0 \\
\hline $\begin{array}{l}\text { Drainable Interstitial } \\
\text { Llquid }\end{array}$ & $\begin{array}{l}3.79 \mathrm{~kL}(1 \\
\mathrm{kgal})\end{array}$ & -0 & $\begin{array}{l}37.9 \mathrm{~kL}(10 \\
\mathrm{kgal})\end{array}$ & $\begin{array}{l}56.8 \mathrm{~kL}(15 \\
\mathrm{kgal})\end{array}$ & $\begin{array}{l}34.1 \mathrm{~kL}(9 \\
\mathrm{kgal})\end{array}$ & $\begin{array}{l}90.8 \mathrm{~kL}(24 \\
\mathrm{kgaD}\end{array}$ \\
\hline Siudge & -0 & -0 & -0 & -0 & -0 & -0 \\
\hline Salt Cake & $\begin{array}{l}132 \mathrm{~kL}(35 \\
\mathrm{kgal})\end{array}$ & $\begin{array}{l}507 \mathrm{~kL}(134 \\
\mathrm{kgal})\end{array}$ & $\begin{array}{l}1,450 \mathrm{~kL}(384 \\
\text { kgal) }\end{array}$ & $\begin{array}{l}1,750 \mathrm{~kL}(462 \\
\text { kgal) }\end{array}$ & $\begin{array}{l}1,400 \mathrm{~kL}(370 \\
\mathrm{kgal})\end{array}$ & $\begin{array}{l}2,460 \mathrm{~kL}(649 \\
\mathrm{kgal})\end{array}$ \\
\hline Integrity Category & ASMD LKR & Sound & Sound & ASMD LKR & Sound & Sound \\
\hline $\begin{array}{l}\text { Leak Vol. Accum. } \\
\text { (last update\} }\end{array}$ & $\begin{array}{l}9.46 \mathrm{~kL}(2.5 \\
\mathrm{kgal}) \\
\{1986\}\end{array}$ & -0 & -0 & $\begin{array}{l}30.3 \mathrm{~kL}(8 \\
\mathrm{kgal})\{1989\}\end{array}$ & -0 & -0 \\
\hline Watch List Status & Not Listed & Not Listed & Not Listed & Not Listed & Not Listed & Not Listed \\
\hline Temperature & $18.9^{\circ} \mathrm{C}\left(66^{\circ} \mathrm{F}\right)$ & $20.0^{\circ} \mathrm{C}\left(68^{\circ} \mathrm{F}\right)$ & $34.4^{\circ} \mathrm{C}\left(94^{\circ} \mathrm{F}\right)$ & o/s & $26.1^{\circ} \mathrm{C}\left(79^{\circ} \mathrm{F}\right)$ & $19.4^{\circ} \mathrm{C}\left(67^{\circ} \mathrm{F}\right)$ \\
\hline Stabil/solation & IS/AP & ISAP & IS $\mathrm{AP}$ & IS $n \mathbf{P}$ & ISAP & ISNP \\
\hline
\end{tabular}


Table 4-12. 241-TX Tank Farm Status.

(sheet 3 of 3 )

\begin{tabular}{|c|c|c|c|c|c|c|}
\hline Iank & $24 \times 1 \times 1 / 3$ & $241 X_{114}$ & $241 / x_{115}$ & $24+1 \times 116$ & 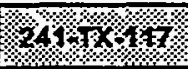 & ${ }_{21} \ln _{1} 18$ \\
\hline Out of Service & 1971 & 1971 & 1977 & 1969 & 1969 & 1980 \\
\hline $\begin{array}{l}\text { Hanford } \\
\text { Coordinates }\end{array}$ & $\begin{array}{l}41,956^{\circ} \mathrm{N} \\
75,750^{\circ} \mathrm{W}\end{array}$ & $\begin{array}{l}41,956^{\circ} \mathrm{N} \\
75,852^{\circ} \mathrm{W}\end{array}$ & $\begin{array}{l}41,956^{\circ} \mathrm{N} \\
75,954^{\circ} \mathrm{W}\end{array}$ & $\begin{array}{l}42,058^{\circ} \mathrm{N} \\
75,750^{\circ} \mathrm{W}\end{array}$ & $\begin{array}{l}42,058^{\circ} \mathrm{N} \\
75,852^{\circ} \mathrm{W}\end{array}$ & $\begin{array}{l}42,058^{\circ} \mathrm{N} \\
75,954^{\circ} \mathrm{W}\end{array}$ \\
\hline Risers, Total & 17 & 21 & 19 & 18 & 16 & 21 \\
\hline FIC Surface Level & No & No & No & No & No & Yes \\
\hline $\begin{array}{l}\text { Manual Tape Surface } \\
\text { Level }\end{array}$ & Yes & Yes & Yes & Yes & Yes & Yes \\
\hline ENRAF Gauge & No & No & No & No & No & No \\
\hline Waste Type & NCPLX & NCPLX & NCPLX & NCPLX & NCPLX & NCPLX \\
\hline Total Waste & $\begin{array}{l}2,300 \mathrm{~kL} \\
(607 \mathrm{kgal})\end{array}$ & $\begin{array}{l}2,030 \mathrm{~kL} \\
(535 \mathrm{kgal})\end{array}$ & $\begin{array}{l}2,420 \mathrm{~kL} \\
(640 \mathrm{kgal})\end{array}$ & $\begin{array}{l}2,390 \mathrm{~kL} \\
(631 \mathrm{kgal})\end{array}$ & $\begin{array}{l}2,370 \mathrm{~kL} \\
(626 \mathrm{kgal})\end{array}$ & $\begin{array}{l}1,310 \mathrm{~kL} \\
(347 \mathrm{kgal})\end{array}$ \\
\hline Supernate & -0 & -0 & -0 & -0 - & -0 & -0 \\
\hline $\begin{array}{l}\text { Drainable Interstitial } \\
\text { Liquid }\end{array}$ & $\begin{array}{l}60.6 \mathrm{~kL}(16 \\
\mathrm{kgal})\end{array}$ & $\begin{array}{l}56.8 \mathrm{~kL}(15 \\
\mathrm{kgal})\end{array}$ & $\begin{array}{l}71.9 \mathrm{~kL}(19 \\
\mathrm{kgal})\end{array}$ & $\begin{array}{l}87.1 \mathrm{~kL}(23 \\
\mathrm{kgal})\end{array}$ & $\begin{array}{l}30.3 \mathrm{~kL}(8 \\
\mathrm{kgal})\end{array}$ & $\begin{array}{l}102 \mathrm{~kL}(27 \\
\mathrm{kgal})\end{array}$ \\
\hline Sludge & -0 & -0 & -0 & -0 & -0 & -0 \\
\hline Salt Cake & $\begin{array}{l}2,300 \mathrm{~kL} \\
(607 \mathrm{kgal})\end{array}$ & $\begin{array}{l}2,030 \mathrm{~kL} \\
\text { (535 kgal) }\end{array}$ & $\begin{array}{l}2,420 \mathrm{~kL} \\
(640 \mathrm{kgal})\end{array}$ & $\begin{array}{l}2,390 \mathrm{~kL} \\
(631 \mathrm{kgal})\end{array}$ & $\begin{array}{l}2,370 \mathrm{~kL} \\
(626 \mathrm{kgal})\end{array}$ & $\begin{array}{l}1,310 \mathrm{~kL} \\
(347 \mathrm{kgal})\end{array}$ \\
\hline Integrity Category & ASMD LKR & ASMD LKR & ASMD LKR & ASMD LKR & ASMD LKR & Sound \\
\hline $\begin{array}{l}\text { Leak Vol. Accum. } \\
\text { \{last update\} }\end{array}$ & $\begin{array}{l}30.3 \mathrm{~kL}(8 \\
\mathrm{kgal}) \\
\{1989\}\end{array}$ & $\begin{array}{l}30.3 \mathrm{~kL}(8 \\
\mathrm{kgal}) \\
\{1989\}\end{array}$ & $\begin{array}{l}30.3 \mathrm{~kL}(8 \\
\mathrm{kgal}) \\
\{1989\}\end{array}$ & $\begin{array}{l}30.3 \mathrm{~kL}(8 \\
\mathrm{kgal})\{1989\}\end{array}$ & $\begin{array}{l}30.3 \mathrm{~kL}(8 \\
\mathrm{kgal})\{1989\}\end{array}$ & -0 \\
\hline Watch List Status & Not Listed & Not Listed & Not Listed & Not Listed & Not Listed & $\begin{array}{l}\text { Ferrocyanide, } \\
\text { Organic Salts }\end{array}$ \\
\hline Temperature & $22.2^{\circ} \mathrm{C}\left(72^{\circ} \mathrm{F}\right)$ & OIS & $21.1^{\circ} \mathrm{C}\left(70^{\circ} \mathrm{F}\right)$ & O/S & O/S & $25.0^{\circ} \mathrm{C}\left(77^{\circ} \mathrm{F}\right)$ \\
\hline Stabil/solation & IS $/ \mathrm{P}$ & IS/IP & IS $/$ P & IS/P & ISIP & IS AP \\
\hline
\end{tabular}




\subsubsection{1-BY Tank Farm}

The 241-BY Tank Farm is located in the 200 East Area. The farm was constructed in 1948-49, using a modified 241-B Tank Farm design (see Figure 4-4). The riser configuration was modified to provide more uniform access to the tank interior. Twelve tanks with capacities of $2,870 \mathrm{~kL}$ (758 kgal) were arranged in four cascades of three tanks each. Each of the cascades are connected to the end cascade tanks of the BX Tank Farm. Tanks 241-BY-101 through -BY106 received $B$ Plant metal wastes in cascade after tanks in the BX Tank Farm were filled. Tanks 241-BY-107 through -BY-110 received B Plant first-cycle waste and TBP waste. Tanks 241-BY111 and $-B Y-112$ were used for temporary storage of metal waste. Tanks 241-BY-104 through $B Y-108$ and $-B Y-110$ received ferrocyanide scavenging waste. Sluicing campaigns for uranium recovery and solidification preparation involved Tanks 241-BY-101 through $-B Y-106,-B Y-111$, and $-B Y-112$. Table 4-13 describes additional information pertaining to the BY Tank Farm. Table 414 provides status of the individual tanks in the BY Tank Farm, including current stabilization status as of March 1994 (Hanlon).

Table 4-13. 241-BY Tank Farm Generic Description.

\begin{tabular}{|l|l|}
\hline Type & S \\
\hline Constructed & $1948-1949$ \\
\hline In Service & $1951(1950:-101,-102,-103,-106,-107,-109)$ \\
\hline Diameter & $22.9 \mathrm{~m}(75 \mathrm{ft})$ \\
\hline Maximum Waste Depth & $7.01 \mathrm{~m}(23 \mathrm{ft})$ \\
\hline Operating Capacity & $2,870,000 \mathrm{~L}(758 \mathrm{kgal})$ \\
\hline Bottom Shape & Dish \\
\hline Ventilation & Passive \\
\hline $\begin{array}{l}\text { External Drywells (each } \\
\text { tank) }\end{array}$ & $\begin{array}{l}5(3:-105,4:-110,-111,6:-107,-109,7:-108,-112,8:- \\
103) 70 \text { total }\end{array}$ \\
\hline Lateral Wells & $-0-$ \\
\hline Airlift Circulators (each) & $-0-$ \\
\hline
\end{tabular}


Table 4-14. 241-BY Tank Farm Status.

(sheet 1 of 2)

\begin{tabular}{|c|c|c|c|c|c|c|}
\hline Pank 1 . & 241811101 & $2418 B Y 102$ & $248 \times 1 \% 03$ & $24 \gamma_{B Y} 104$ & 24183405 & $24183 \times 106 \%$ \\
\hline Out of Service & 1971 & 1977 & 1973 & 1977 & 1974 & 1977 \\
\hline $\begin{array}{l}\text { Hanford } \\
\text { Coordinates }\end{array}$ & $\begin{array}{l}45,898^{\circ} \mathrm{N} \\
53,247^{\circ} \mathrm{W}\end{array}$ & $\begin{array}{l}46,000^{\circ} \mathrm{N} \\
53,247^{\circ} \mathrm{W}\end{array}$ & $\begin{array}{l}46,102^{\circ} \mathrm{N} \\
53,247^{\circ} \mathrm{W}\end{array}$ & $\begin{array}{l}45,898^{\circ} \mathrm{N} \\
53,349 \% \mathrm{~W}\end{array}$ & $\begin{array}{l}46,000^{\circ} \mathrm{N} \\
53,349^{\circ} \mathrm{W}\end{array}$ & $\begin{array}{l}46,102^{\circ} \mathrm{N} \\
53,349^{\circ} \mathrm{W}\end{array}$ \\
\hline Risers, Total & 18 & 17 & 22 & 15 & 19 & 18 \\
\hline FIC Surface Level & No & No & No & No & No & No \\
\hline $\begin{array}{l}\text { Manual Tape Surface } \\
\text { Level }\end{array}$ & Yes & Yes & Yes & Yes & Yes & Yes \\
\hline ENRAF Gauge & No & No & No & No & No & No \\
\hline Waste Type & NCPLX & NCPLX & NCPLX & NCPLX & NCPLX & NCPLX \\
\hline Total Waste & $\begin{array}{l}1,460 \mathrm{~kL} \\
(387 \mathrm{kgal})\end{array}$ & $\begin{array}{l}1,290 \mathrm{~kL} \\
(341 \mathrm{kgal})\end{array}$ & $\begin{array}{l}1,510 \mathrm{~kL} \\
(400 \mathrm{kgal})\end{array}$ & $\begin{array}{l}1,540 \mathrm{~kL} \\
(406 \mathrm{kgal})\end{array}$ & $\begin{array}{l}1,900 \mathrm{~kL} \\
(503 \mathrm{kgal})\end{array}$ & $\begin{array}{l}2,430 \mathrm{~kL} \\
(642 \mathrm{kgal})\end{array}$ \\
\hline Supernate & -0 & -0 & -0 & -0 & -0 & -0 \\
\hline $\begin{array}{l}\text { Drainable Interstitial } \\
\text { Liquid }\end{array}$ & $\begin{array}{l}18.9 \mathrm{~kL}(5 \\
\mathrm{kgal})\end{array}$ & $\begin{array}{l}155 \mathrm{~kL}(41 \\
\mathrm{kgal})\end{array}$ & $\begin{array}{l}606 \mathrm{~kL}(160 \\
\mathrm{kgal})\end{array}$ & $\begin{array}{l}68.1 \mathrm{~kL}(18 \\
\mathrm{kgal})\end{array}$ & $\begin{array}{l}727 \mathrm{~kL}(192 \\
\text { kgal) }\end{array}$ & $\begin{array}{l}890 \mathrm{~kL}(235 \\
\mathrm{kgal})\end{array}$ \\
\hline Sludge & $\begin{array}{l}413 \mathrm{~kL} \mathrm{(109} \\
\mathrm{kgal})\end{array}$ & -0 & $\begin{array}{l}18.9 \mathrm{~kL}(5 \\
\mathrm{kgal})\end{array}$ & $\begin{array}{l}151 \mathrm{~kL}(40 \\
\mathrm{kgal})\end{array}$ & $\begin{array}{l}167 \mathrm{~kL} \mathrm{(44} \\
\mathrm{kgal})\end{array}$ & $\begin{array}{l}360 \mathrm{~kL}(95 \\
\mathrm{kgal})\end{array}$ \\
\hline Salt Cake & $\begin{array}{l}1,050 \mathrm{~kL} \\
(278 \mathrm{kgal})\end{array}$ & $\begin{array}{l}1,290 \mathrm{~kL} \\
\text { (341 kgal) }\end{array}$ & $\begin{array}{l}1,500 \mathrm{~kL} \\
(395 \mathrm{kgal})\end{array}$ & $\begin{array}{l}1,390 \mathrm{~kL} \\
\text { (366 kgal) }\end{array}$ & $\begin{array}{l}1,740 \mathrm{~kL} \\
(459 \mathrm{kgal})\end{array}$ & $\begin{array}{l}2,070 \mathrm{~kL} \\
(547 \mathrm{kgal})\end{array}$ \\
\hline Integrity Category & Sound & Sound & ASMD LKR & Sound & ASMD LKR & ASMD LKR \\
\hline $\begin{array}{l}\text { Leak Vol. Accum. } \\
\text { \{last update\} }\end{array}$ & -0 & -0 & $\begin{array}{l}<18.9 \mathrm{~kL} \\
(<5 \mathrm{kgal}) \\
\{1983\}\end{array}$ & -0 & $\begin{array}{l}30.3 \mathrm{~kL} \\
(8 \mathrm{kgal}) \\
\{1989\}\end{array}$ & $\begin{array}{l}30.3 \mathrm{~kL} \\
(8 \mathrm{kgal}) \\
\{1989\}\end{array}$ \\
\hline Watch List Status & Not Listed & Not Listed & Ferrocyanide & Ferrocyanide & Ferrocyanide & Ferrocyanide \\
\hline $\begin{array}{c}\text { Temperature } \\
=\end{array}$ & $\begin{array}{l}23.9^{\circ} \mathrm{C} \\
\left(75^{\circ} \mathrm{F}\right)\end{array}$ & $\begin{array}{l}\text { O/S } \\
22.2^{\circ} \mathrm{C}\left(72^{\circ} \mathrm{F}\right) \\
4 / 79\end{array}$ & $\begin{array}{l}27.8^{\circ} \mathrm{C} \\
\left(82^{\circ} \mathrm{F}\right)\end{array}$ & $\begin{array}{l}53.3^{\circ} \mathrm{C} \\
\left(128^{\circ} \mathrm{F}\right)\end{array}$ & $\begin{array}{l}48.9^{\circ} \mathrm{C} \\
\left(120^{\circ} \mathrm{F}\right)\end{array}$ & $\begin{array}{l}53.9^{\circ} \mathrm{C} \\
\left(129^{\circ} \mathrm{F}\right)\end{array}$ \\
\hline Stabil/solation & IS/IP & $/ \mathrm{PI}$ & $|P|$ & IS/IP & IPI & IPI \\
\hline
\end{tabular}


Table 4-14. 241-BY Tank Farm Status.

(sheet 2 of 2)

\begin{tabular}{|c|c|c|c|c|c|c|}
\hline Tunk & $2413 \mathrm{y}$ 107. & $248 \% 108 \%$ & $2418 \mathrm{ar} \% 109$ & $2783 y+10$ & 244 BY, 13 & $21581 / 12$ \\
\hline Out of Service & 1974 & 1972 & 1979 & 1979 & 1977 & 1978 \\
\hline $\begin{array}{l}\text { Hanford } \\
\text { Coordinates }\end{array}$ & $\begin{array}{l}45,898^{\circ} \mathrm{N} \\
53,451^{\circ} \mathrm{W}\end{array}$ & $\begin{array}{l}46,000 \% \mathrm{~N} \\
53,451^{\prime} \mathrm{W}\end{array}$ & $\begin{array}{l}46,102^{\circ} \mathrm{N} \\
53,451^{\mathrm{W}} \mathrm{W}\end{array}$ & $\begin{array}{l}45,898^{\circ} \mathrm{N} \\
53,553^{\circ} \mathrm{W}\end{array}$ & $\begin{array}{l}46,000 \cdot N \\
53,553 \% W\end{array}$ & $\begin{array}{l}46,102^{\circ} \mathrm{N} \\
53,553^{\circ} \mathrm{W}\end{array}$ \\
\hline Risers, Total & 17 & 17 & 19 & 16 & 19 & 21 \\
\hline FIC Surface Level & No & No & No & No & No & No \\
\hline $\begin{array}{l}\text { Manual Tape Surface } \\
\text { Level }\end{array}$ & Yes & Yes & Yes & Yes & $\dot{\text { Yes }}$ & Yes. \\
\hline ENRAF Gauge & No & No & No & No & No & No \\
\hline Waste Type & NCPLX & NCPLX & NCPLX & NCPLX & NCPLX & NCPLX \\
\hline Total Waste & $\begin{array}{l}1,010 \mathrm{~kL} \\
(266 \mathrm{kgal})\end{array}$ & $\begin{array}{l}863 \mathrm{~kL} \\
(228 \mathrm{kgal})\end{array}$ & $\begin{array}{l}1,600 \mathrm{~kL} \\
(423 \mathrm{kgal})\end{array}$ & $\begin{array}{l}1,510 \mathrm{~kL} \\
(398 \mathrm{kgal})\end{array}$ & $\begin{array}{l}1,740 \mathrm{~kL} \\
(459 \mathrm{kgal})\end{array}$ & $\begin{array}{l}1,100 \mathrm{~kL} \\
(291 \mathrm{kgal})\end{array}$ \\
\hline Supernate & -0 & -0 & -0 & -0 & -0 & -0 \\
\hline $\begin{array}{l}\text { Drainable Interstitial } \\
\text { Liquid }\end{array}$ & $\begin{array}{l}94.6 \mathrm{~kL}(25 \\
\text { kgal) }\end{array}$ & $34.1 \mathrm{~kL}(9 \mathrm{kgal})$ & $\begin{array}{l}295 \mathrm{~kL}(78 \\
\mathrm{kgal})\end{array}$ & $\begin{array}{l}34.1 \mathrm{~kL}(9 \\
\text { kgal) }\end{array}$ & -0 & $\begin{array}{l}30.3 \mathrm{~kL}(8 \\
\mathrm{kgal})\end{array}$ \\
\hline Sludge & $\begin{array}{l}227 \mathrm{~kL}(60 \\
\mathrm{kgal})\end{array}$ & $\begin{array}{l}583 \mathrm{~kL}(154 \\
\mathrm{kgal})\end{array}$ & $\begin{array}{l}314 \mathrm{~kL}(83 \\
\mathrm{kgal})\end{array}$ & $\begin{array}{l}390 \mathrm{~kL}(103 \\
\mathrm{kgal})\end{array}$ & $\begin{array}{l}79.5 \mathrm{~kL}(21 \\
\mathrm{kgal})\end{array}$ & $\begin{array}{l}18.9 \mathrm{~kL}(5 \\
\mathrm{kgal})\end{array}$ \\
\hline Salt Cake & $\begin{array}{l}780 \mathrm{~kL} \\
(206 \mathrm{kgal})\end{array}$ & $\begin{array}{l}280 \mathrm{~kL} \\
(74 \mathrm{kgal})\end{array}$ & $\begin{array}{l}1,290 \mathrm{~kL} \\
(340 \mathrm{kgal})\end{array}$ & $\begin{array}{l}1,120 \mathrm{~kL} \\
(295 \mathrm{kgal})\end{array}$ & $\begin{array}{l}1,660 \mathrm{~kL} \\
(438 \mathrm{kgal})\end{array}$ & $\begin{array}{l}2,460 \mathrm{~kL} \\
(649 \mathrm{kgal})\end{array}$ \\
\hline Integrity Category & ASMD LKR & ASMD LKR & Sound & Sound & Sound & Sound \\
\hline $\begin{array}{l}\text { Leak Vol. Accum. } \\
\text { (last update\} }\end{array}$ & $\begin{array}{l}57.2 \mathrm{~kL} \quad(15.1 \\
\mathrm{kgal}) \quad\{1989\}\end{array}$ & $\begin{array}{l}<18.9 \mathrm{~kL}<5 \\
\mathrm{kgal})\{1983\}\end{array}$ & -0 & -0 & -0 & -0 \\
\hline Watch List Status & Ferrocyanide & Ferrocyanide & Not Listed & Ferrocyanide & Ferrocyanide & Ferrocyanide \\
\hline Temperature & $34.4^{\circ} \mathrm{C} \quad\left(94^{\circ} \mathrm{F}\right)$ & $43.3^{\circ} \mathrm{C} \quad\left(110^{\circ} \mathrm{F}\right)$ & O/S & $\begin{array}{l}47.8^{\circ} \mathrm{C} \\
\left(118^{\circ} \mathrm{F}\right)\end{array}$ & $\begin{array}{l}31.1^{\circ} \mathrm{C} \\
\left(88^{\circ} \mathrm{F}\right)\end{array}$ & $\begin{array}{l}32.2^{\circ} \mathrm{C} \\
\left(90^{\circ} \mathrm{F}\right)\end{array}$ \\
\hline Stabil/solation & IS/IP & IS/RP & IPI & IS/IP & IS/PP & ISnP \\
\hline
\end{tabular}




\subsubsection{1-S Tank Farm}

The 241-S Tank Farm is located in the 200 West Area. This tank farm was constructed in 1950-51, using a second generation tank design (see Figure 4-4). The configuration is similar to the original 241-B Tank Farm design, but with an increased operating depth. Twelve tanks with capacities of $2,870 \mathrm{~kL}$ (758 kgal) are arranged in four cascades of three tanks each. The $S$ Tank Farm received several types of REDOX wastes in all four cascades. In 1952 the waste in some of the tanks began to boil spontaneously. Surface condensers were installed in the first two cascades to self-concentrate the wastes. The vapor condensate was disposed through cribbing. Additional concentration was achieved in the S Tank Farm using the 242-S Evaporator. Table 415 describes additional information pertaining to the $S$ Tank Farm. Table 4-16 provides status of the individual tanks in the 241-S Tank Farm, including current stabilization status as of March 1994 (Hanlon).

Table 4-15. 241-S Tank Farm Generic Description.

\begin{tabular}{|c|c|}
\hline Hoplow & \%ै\% \\
\hline Constructed & $1958-61$ \\
\hline In Service & 1952 (1953: -101 through -106) \\
\hline Diameter & $22.9 \mathrm{~m}(75 \mathrm{ft})$ \\
\hline Maximum Waste Depth & $7.01 \mathrm{~m}(23 \mathrm{ft})$ \\
\hline Operating Capacity & $2,870,000 \mathrm{~L}(758 \mathrm{kgal})$ \\
\hline Bottom Shape & Dish \\
\hline Ventilation & Passive \\
\hline $\begin{array}{l}\text { External Drywells (each } \\
\text { tank) }\end{array}$ & $\begin{array}{l}5(4:-104,6:-106,-107,-103,7:-103,-110,8:- \\
\text { 102) } 72 \text { total }\end{array}$ \\
\hline Lateral Wells & -0 \\
\hline Airlift Circulators (each) & -0 \\
\hline
\end{tabular}


Table 4-16. 241-S Tank Farm Status.

(sheet 1 of 2)

\begin{tabular}{|c|c|c|c|c|c|c|}
\hline hank & $2415101 \%$ & 2445102 & $244510 s$ & $244510 \%$ & 2416105 & 245106 \\
\hline Out of Service & 1980 & 1980 & 1980 & 1968 & 1974 & 1979 \\
\hline $\begin{array}{l}\text { Hanford } \\
\text { Coordinates }\end{array}$ & $\begin{array}{l}36,226^{\circ} \mathrm{N} \\
75,665^{\circ} \mathrm{W}\end{array}$ & $\begin{array}{l}36,226^{\circ} \mathrm{N} \\
75,767^{\circ} \mathrm{W}\end{array}$ & $\begin{array}{l}36,226^{\circ} \mathrm{N} \\
75,869 \% \mathrm{~W}\end{array}$ & $\begin{array}{l}36,124^{\circ} \mathrm{N} \\
75,665^{\circ} \mathrm{W}\end{array}$ & $\begin{array}{l}36,124^{\circ} \mathrm{N} \\
75,767^{\circ} \mathrm{W}\end{array}$ & $\begin{array}{l}36,124^{\circ} \mathrm{N} \\
75,869^{\circ} \mathrm{W}\end{array}$ \\
\hline Rlsers, Total & 12 & 12 & 12 & 9 & 12 & 12 \\
\hline FIC Surface Level & Yes & Yes & Yes & Yes & Yes & Yes \\
\hline $\begin{array}{l}\text { Manual Tape } \\
\text { Surface Level }\end{array}$ & No & No & No & No & No & No \\
\hline ENRAF Gauge & No & No & Yes & No & No. & Yes \\
\hline Waste Type & NCPLX & DSSF & DSSF & NCPLX & NCPLX & NCPLX \\
\hline Total Waste & $\begin{array}{l}1,620 \mathrm{~kL} \quad(427 \\
\mathrm{kgal})\end{array}$ & $\begin{array}{l}2,080 \text { kL (549 } \\
\text { kgal) }\end{array}$ & $\begin{array}{l}939 \mathrm{~kL} \quad(248 \\
\mathrm{kgal})\end{array}$ & $\begin{array}{l}1,110 \mathrm{~kL}(294 \\
\mathrm{kgal})\end{array}$ & $\begin{array}{l}1,730 \mathrm{~kL} \text { (456 } \\
\text { kgai) }\end{array}$ & $\begin{array}{l}1,810 \mathrm{~kL} \\
(479 \mathrm{kgai})\end{array}$ \\
\hline Supernate & $\begin{array}{l}45.4 \mathrm{~kL}(12 \\
\mathrm{kgal})\end{array}$ & -0 & $\begin{array}{l}64.4 \mathrm{~kL} \mathrm{(17} \\
\mathrm{kgal})\end{array}$ & $\begin{array}{l}3.79 \mathrm{~kL}(1 \\
\mathrm{kgal})\end{array}$ & -0 & $\begin{array}{l}15.1 \mathrm{~kL}(4 \\
\mathrm{kgal})\end{array}$ \\
\hline $\begin{array}{l}\text { Drainable Interstitial } \\
\text { Liquild }\end{array}$ & $\begin{array}{l}318 \mathrm{~kL}(84 \\
\mathrm{kgal})\end{array}$ & $\begin{array}{l}871 \mathrm{~kL}(230 \\
\mathrm{kgal})\end{array}$ & $\begin{array}{l}322 \mathrm{~kL}(85 \\
\mathrm{kgal})\end{array}$ & $\begin{array}{l}106 \mathrm{~kL}(28 \\
\mathrm{kgal})\end{array}$ & $\begin{array}{l}132 \mathrm{~kL} \mathrm{(35} \\
\mathrm{kgal})\end{array}$ & $\begin{array}{l}704 \mathrm{~kL}(186 \\
\mathrm{kgal})\end{array}$ \\
\hline Sludge & $\begin{array}{l}924 \mathrm{~kL}(244 \\
\text { kgal) }\end{array}$ & $\begin{array}{l}15.1 \mathrm{~kL}(4 \\
\mathrm{kgal})\end{array}$ & $\begin{array}{l}37.9 \mathrm{~kL}(10 \\
\mathrm{kgal})\end{array}$ & $\begin{array}{l}1,110 \mathrm{~kL}(293 \\
\mathrm{kgal})\end{array}$ & $\begin{array}{l}7.57 \mathrm{~kL}(2 \\
\mathrm{kgal})\end{array}$ & $\begin{array}{l}106 \mathrm{~kL} \text { (28 } \\
\mathrm{kgal})\end{array}$ \\
\hline Salt Cake & $\begin{array}{l}647 \mathrm{~kL} \quad(171 \\
\mathrm{kgal})\end{array}$ & $\begin{array}{l}2,060 \text { kL (545 } \\
\text { kgal) }\end{array}$ & $\begin{array}{l}837 \mathrm{~kL} \quad(221 \\
\mathrm{kgal})\end{array}$ & -0 & $\begin{array}{l}1,720 \mathrm{~kL} \quad(454 \\
\mathrm{kgal})\end{array}$ & $\begin{array}{l}1,690 \mathrm{~kL} \\
(447 \mathrm{kgal})\end{array}$ \\
\hline Integrity Category & Sound & Sound & Sound & ASMD LKR & Sound & Sound \\
\hline $\begin{array}{l}\text { Leak Vol. Accum. } \\
\text { \{last update\} }\end{array}$ & -0. & -0 & -0 & $\begin{array}{l}90.8 \mathrm{~kL} \mathrm{(24} \\
\mathrm{kgal}) \\
\{1989\}\end{array}$ & -0 & -0 \\
\hline Watch List Status & Not Listed & $\begin{array}{l}\text { Hydrogen, } \\
\text { Organic Salts }\end{array}$ & Not Listed & Not Listed & Not Listed & Not Listed \\
\hline Tempērature & $38.3^{\circ} \mathrm{C}\left(101^{\circ} \mathrm{F}\right)$ & $42.2 \mathrm{C}\left(108^{\circ} \mathrm{F}\right)$ & $30.6^{\circ} \mathrm{C}\left(87^{\circ} \mathrm{F}\right)$ & $42.2^{\circ} \mathrm{C}\left(108^{\circ} \mathrm{F}\right)$ & $25.6^{\circ} \mathrm{C}\left(78^{\circ} \mathrm{F}\right)$ & $\begin{array}{l}27.2^{\circ} \mathrm{C} \\
\left(81^{\circ} \mathrm{F}\right)\end{array}$ \\
\hline Stabil/isolation & $/ \mathrm{PI}$ & /PI & IPI & IS $/ \mathrm{P}$ & IS/IP & IPI \\
\hline
\end{tabular}


Table 4-16. 241-S Tank Farm Status.

(sheet 2 of 2)

\begin{tabular}{|c|c|c|c|c|c|c|}
\hline $\sinh$ & $240 \leq 807$ & $24,5=108$ & 24155109 & $22105110=1$ & 24ts=11: & 2415112 \\
\hline Out of Service & 1980 & 1979 & 1979 & 1979 & 1972 & 1974 \\
\hline $\begin{array}{l}\text { Hanford } \\
\text { Coordinates }\end{array}$ & $\begin{array}{l}36,022^{\circ} \mathrm{N} \\
75,665^{\circ} \mathrm{W}\end{array}$ & $\begin{array}{l}36,022^{\circ} \mathrm{N} \\
75,767^{\circ} \mathrm{W}\end{array}$ & $\begin{array}{l}36,022 \% W \\
75,869 \%\end{array}$ & $\begin{array}{l}35,920^{\circ} \mathrm{N} \\
75,665^{\circ} \mathrm{W}\end{array}$ & $\begin{array}{l}35,920^{\circ} \mathrm{N} \\
75,767^{\circ} \mathrm{W}\end{array}$ & $\begin{array}{l}35,920^{\circ} \mathrm{N} \\
75,869 \times W\end{array}$ \\
\hline Risers, Total & 12 & 12 & 12 & 12 & 12 & 12 \\
\hline $\begin{array}{l}\text { FIC Surface } \\
\text { Level }\end{array}$ & Yes & Yes & Yes & Yes & Yes & Yes \\
\hline $\begin{array}{l}\text { Manual Tape } \\
\text { Surface Level }\end{array}$ & No. & Yes & No & No & No & No \\
\hline ENRAF Gauge & Yes & No & No & No & Yes & No \\
\hline Waste Type & NCPLX & NCPLX & NCPLX & NCPLX & NCPLX & NCPLX \\
\hline Total Waste & $\begin{array}{l}1,420 \mathrm{~kL} \text { (376 } \\
\text { kgal) }\end{array}$ & $\begin{array}{l}2,290 \mathrm{~kL}(604 \\
\text { kgal) }\end{array}$ & $\begin{array}{l}2,150 \mathrm{~kL}(568 \\
\mathrm{kgal})\end{array}$ & $\begin{array}{l}1,480 \mathrm{~kL}(390 \\
\mathrm{kgal})\end{array}$ & $\begin{array}{l}2,260 \mathrm{~kL} \text { (596 } \\
\text { kgal) }\end{array}$ & $\begin{array}{l}1,980 \mathrm{~kL}(523 \\
\mathrm{kgal})\end{array}$ \\
\hline Supernate & $\begin{array}{l}53.0 \mathrm{~kL} \text { (14 } \\
\mathrm{kgal})\end{array}$ & 0 & -0 & -0 & $\begin{array}{l}37.9 \mathrm{~kL}(10 \\
\mathrm{kgal})\end{array}$ & -0 \\
\hline $\begin{array}{l}\text { Drainable } \\
\text { Interstitial } \\
\text { Liquid }\end{array}$ & $\begin{array}{l}170 \mathrm{~kL} \mathrm{(45} \\
\mathrm{kgal})\end{array}$ & $\begin{array}{l}481 \mathrm{~kL}(127 \\
\mathrm{kgal})\end{array}$ & $\begin{array}{l}534 \mathrm{~kL}(141 \\
\mathrm{kgal})\end{array}$ & $\begin{array}{l}416 \mathrm{~kL}(110 \\
\text { kgal) }\end{array}$ & $\begin{array}{l}738 \mathrm{~kL} \text { (195 } \\
\text { kgal) }\end{array}$ & $\begin{array}{l}416 \mathrm{~kL}(110 \\
\mathrm{kgal})\end{array}$ \\
\hline Sludge & $\begin{array}{l}1,110 \mathrm{~kL}(293 \\
\text { kgal) }\end{array}$ & $\begin{array}{l}15.1 \text { kL (4 } \\
\text { kgal) }\end{array}$ & $\begin{array}{l}49.2 \mathrm{~kL}(13 \\
\mathrm{kgal})\end{array}$ & $\begin{array}{l}496 \mathrm{~kL}(131 \\
\text { kgal) }\end{array}$ & $\begin{array}{l}526 \text { kL (139 } \\
\text { kgal) }\end{array}$ & $\begin{array}{l}18.9 \mathrm{~kL}(5 \\
\mathrm{kgal})\end{array}$ \\
\hline Salt Cake & $\begin{array}{l}261 \mathrm{~kL}(69 \\
\text { kgal) }\end{array}$ & $\begin{array}{l}2,270 \mathrm{KL}(600 \\
\text { kgal) }\end{array}$ & $\begin{array}{l}2,100 \mathrm{~kL}(555 \\
\mathrm{kgal})\end{array}$ & $\begin{array}{l}980 \mathrm{~kL} \text { (259 } \\
\mathrm{kgal})\end{array}$ & $\begin{array}{l}\text { 1,690 kL (447 } \\
\text { kgal) }\end{array}$ & $\begin{array}{l}1,960 \mathrm{~kL}(518 \\
\mathrm{kgal})\end{array}$ \\
\hline $\begin{array}{l}\text { Integrity } \\
\text { Category }\end{array}$ & Sound & Sound & Sound & Sound & Sound & Sound \\
\hline $\begin{array}{l}\text { Leak Vol. } \\
\text { Accum. } \\
\text { \{last update\} }\end{array}$ & -0 & -0 & -0 & -0 & -0 & -0 \\
\hline $\begin{array}{l}\text { Watch List } \\
\text { Status }\end{array}$ & Not Listed & Not Listed & Not Listed & Not Listed & Hydrogen & Hydrogen \\
\hline Temperature & $43.3^{\circ} \mathrm{C}\left(110^{\circ} \mathrm{F}\right)$ & $31.7^{\circ} \mathrm{C}\left(89^{\circ} \mathrm{F}\right)$ & $20.0^{\circ} \mathrm{C}\left(68^{\circ} \mathrm{F}\right)$ & $47.2^{\circ} \mathrm{C}\left(117^{\circ} \mathrm{F}\right)$ & $33.9^{\circ} \mathrm{C}\left(93^{\circ} \mathrm{F}\right)$ & $31.1^{\circ} \mathrm{C}\left(88^{\circ} \mathrm{F}\right)$ \\
\hline Stabi//solation & IPI & IPI & /PI & $\mathbb{P} \mathbf{P I}$ & IPI & IPI \\
\hline
\end{tabular}




\subsubsection{1-TY Tank Farm}

The 241-TY Tank Farm is located in the 200 West Area. This farm, with six tanks, was constructed in 1951-52, using the same design as the 241-S Tank Farm tanks (see Figure 4-4). The TY Tank Farm tanks were used to settle and decant low-level wastes and as lag storage for the 242-T Evaporator. Tank TY-106 contains diatomaceous earth. Table 4-17 describes additional information pertaining to the TY Tank Farm. Table 4-18 provides status of the individual tanks in the TY Tank Farm, including current. stabilization status as of March 1994 (Hanion).

Table 4-17. 241-TY Tank Farm Generic Description.

\begin{tabular}{|c|c|}
\hline Wyph & olokishil $\%$ \\
\hline Constructed & 1950.51 \\
\hline In Service & 1953 \\
\hline Dlemoter & $22.9 m(75 \mathrm{ft})$ \\
\hline Maxinum Waste Depth & $7.01 \mathrm{~m}(23 \mathrm{tt})$ \\
\hline Operating Capaclty & $2,870,000$ L (758 kgal) \\
\hline Bottom Shapo & Diah \\
\hline Ventilation & Passive \\
\hline External Drywelis (oech tank) & $5(1:-105,3:-101,-103)$ \\
\hline Lateral Wolle & - \\
\hline Airlift Circulator (onch) & - \\
\hline
\end{tabular}

Table 4-18. 241-TY Tank Farm Status.

\begin{tabular}{|c|c|c|c|c|c|c|}
\hline thp & $241+100 \%$ & 41-TY 102 \% & $2 \mathrm{for}_{0} \mathrm{~s}$ & $341,4, \alpha)$ & $411-1,105$ & $241+4>100$ \\
\hline Out of Service & 1873 & .978 & 1976 & 1974 & 1960 & 1977 \\
\hline $\begin{array}{l}\text { Hentord } \\
\text { Coordlinetes }\end{array}$ & $\begin{array}{l}42,602^{\circ} \mathrm{K} \\
75,852^{\circ} \mathrm{W}\end{array}$ & $\begin{array}{l}42,602 \% \mathrm{~W} \\
75,954 \%\end{array}$ & $\begin{array}{l}42,500^{\circ} \mathrm{W} \\
75,852^{\circ} \mathrm{W}\end{array}$ & $\begin{array}{l}42,500^{\circ} \mathrm{K} \\
75,954 \% \mathrm{~W}\end{array}$ & $\begin{array}{l}42,400^{\circ} \mathrm{K} \\
75,852^{\circ} \mathrm{W}\end{array}$ & $\begin{array}{l}42,400^{\circ} \mathrm{H} \\
75,954 \times \mathrm{Y}\end{array}$ \\
\hline Risere, Total & 17 & 15 & 14 & 13 & 11 & 10 \\
\hline FIC surface Level & Yes & Yea & Yos & Yes & Yes & Yee \\
\hline Manual Tape Surface Leval & Ho & No & Ho & Ho & Yes & Yos \\
\hline ENRAF Gaugo & Ho & Ho & Ho & No & No & No \\
\hline Waste Trpo & NCPLX & NCPLX & HCPLX & HCPLX & NCPLX & MCPLX \\
\hline Total Waste & $447 \mathrm{~kL}$ (118 kgal) & $242 \mathrm{~kL}(84 \mathrm{kgal})$ & 813 kL (162 kgal) & $174 \mathrm{~kL}$ (46 kgal) & $874 \mathrm{~kL}$ (231 kgan) & $04.4 \mathrm{~kL}$ (17 kgal) \\
\hline Supernate & -0 & 6 & - & $49.2 \mathrm{~kL}$ (13 kgal) & $-\infty$ & -0 . \\
\hline Drainable Interattist Liquid & $\infty$ & $53.0 \mathrm{~kL}(14 \mathrm{kgal})$ & $18.8 \mathrm{~kL}(5 \mathrm{kgal})$ & $45.4 \mathrm{~kL}$ (12 kgal) & $\infty$ & -0 - \\
\hline Sludge & $447 \mathrm{~kL}$ (118 kgal) & -0 & $613 \mathrm{~kL}$ (162 kgal) & $163 \mathrm{~kL}$ (43 kgal) & $874 \mathrm{~kL}$ (231 kgal) & $64.4 \mathrm{~kL}$ (17 kgal) \\
\hline Salt Cake & -0 & $242 \mathrm{~kL}$ (E4 kgal) & -0 & $\infty$ & -0 & -0 \\
\hline Integrity Category & ASMD LKR & Sound & ASHD LKR & ASMD LKR & ASMD LKR & ASMD LKR \\
\hline $\begin{array}{l}\text { Leak Vol. Accum, } \\
\text { (lest update) }\end{array}$ & $\begin{array}{l}<3.79 \mathrm{~kL} \\
(<1 \mathrm{~kg}+1)(1880)\end{array}$ & -0. & $\begin{array}{l}11.4 \mathrm{~kL} \\
(3 \mathrm{kgal}) \text { (1886) }\end{array}$ & $\begin{array}{l}5.30 \mathrm{~kL} \\
(1.4 \mathrm{~kg}=1)(1886\}\end{array}$ & $\begin{array}{l}132 \mathrm{~kL} \\
(35 \mathrm{kgal}) \text { (1986\} }\end{array}$ & $\begin{array}{l}75.7 \mathrm{~kL} \\
(20 \mathrm{~kg}=1)(1986)\end{array}$ \\
\hline Watch Llat Statu: & Ferrocyanide & Not Listed & Forrocyanide & Forrocyanide & Not Liated & Not Listed \\
\hline Temperature & $18.9^{\circ} \mathrm{C}\left\{66^{\circ} \mathrm{F}\right\}$ & $15.6 C\left(60^{\circ} \mathrm{F}\right)$ & $20.6^{\circ} \mathrm{C}\left(69^{\circ} \mathrm{F}\right)$ & $18.9^{\circ} \mathrm{C}\left(65^{\circ} \mathrm{F}\right)$ & $26.1^{\circ} \mathrm{C}\left(78^{\circ} \mathrm{F}\right)$ & $15.0^{\circ} \mathrm{C}\left(59^{\circ} \mathrm{F}\right)$ \\
\hline Stably)eofation & ISAP & ISnP & $15 n P$ & ISAP & $1 S n P$ & isnP \\
\hline
\end{tabular}




\subsubsection{1-sX Tank Farm}

The 241-SX Tank Farm is located in the 200 West Area. This farm, with 15 tanks, was constructed in 1951-52. These tanks are a third generation dished-bottom design with capacities of $3,790 \mathrm{~kL}(1,000 \mathrm{kgal}$ )(see Figure 4-5). The tanks were designed to accommodate self-boiling waste with maximum temperatures of $121^{\circ} \mathrm{C}\left(250^{\circ} \mathrm{F}\right)$ and boiling periods of 1 to 5 years. The tanks were configured in five cascades with overflow waste from the end tanks designed to overflow into cribs. Tanks 241-SX-101 through -SX-106 were vented through the condenserventilation system of Tank SX-109 via an underground duct network. Tanks 241-SX-107 through $-S X-112$ and $-S X-114$ were connected to an above ground exhauster system. The first six tanks received REDOX Plant salt waste and first-cycle condensate. After the waste in the tanks had self-concentrated for several years, the tanks were used to support operation of the 242-S Evaporator as bottoms and receiver tanks. In 1955 the remaining nine tanks received REDOX boiling high-level waste and salt waste. Due to the high heat load on the ventilation system, condensate was refluxed back to the tanks to maintain an even cooling rate. Airlift circulators were used to mix tank contents and prevent bumping. Table 4-19 describes additional information pertaining to the SX Tank Farm. Table 4-20 provides status of the individual tanks in the SX Tank Farm, including current stabilization status as of March 1994 (Hanlon).

Table 4-19. 241-SX Tank Farm Generic Description.

\begin{tabular}{|c|c|}
\hline Туре & 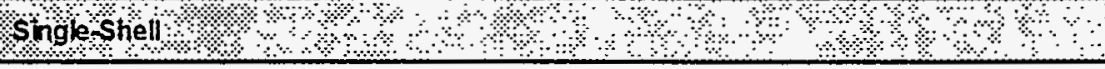 \\
\hline Constructed & $1953-54$ \\
\hline In Service & $\begin{array}{l}\text { 1954: }-101 \text { to }-103,-104,1955:-104,-105,-108,-109,1956:-107,-111,-112,-114 \text {, } \\
\text { 1958: }-113,-115,1960:-110\end{array}$ \\
\hline Diameter & $22.9 m(75 \mathrm{ft})$ \\
\hline Maximum Waste Depth & $9.42 \mathrm{~m}(30.9 \mathrm{ft})$ \\
\hline Operating Capacity & $3,790,000 \mathrm{~L}(1,000 \mathrm{kgal})$ \\
\hline Bottom Shape & Dish \\
\hline Ventilation & Operating Exhauster (Passive $-113,-115$ ) \\
\hline External Drywells (each tank) & $7(1:-113,3:-102,6:-103,-104,-106,-108,-115,8:-110) \quad 98$ total \\
\hline Lateral Wells & 3 \\
\hline
\end{tabular}


Table 4-20. 241-SX Tank Farm Status. (sheet 1 of 3)

\begin{tabular}{|c|c|c|c|c|c|}
\hline thn & 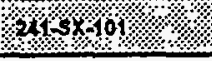 & 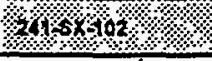 & 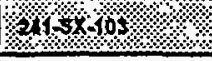 & $248 \times 101$ & 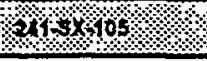 \\
\hline Out of Service & 1080 & 1980 & 1880 & 1080 & 1080 \\
\hline $\begin{array}{l}\text { Hunford } \\
\text { Coordinatos }\end{array}$ & $\begin{array}{l}35,652^{\circ} \mathrm{N} \\
75,665^{\circ} \mathrm{W}\end{array}$ & $\begin{array}{l}35,552^{\circ} \mathrm{K} \\
\mathrm{T}, 787^{*} \mathrm{~W}\end{array}$ & $\begin{array}{l}35,552^{\circ} \mathrm{H} \\
75,889 \% \mathrm{~W}\end{array}$ & $\begin{array}{l}35,450^{\circ} \mathrm{N} \\
78,663^{*} \mathrm{~W}\end{array}$ & $\begin{array}{l}35,450 \% \mathrm{~W} \\
76,767 \% \mathrm{~W}\end{array}$ \\
\hline Rizere, Total & 17 & 13 & 13 & 12 & 20 \\
\hline Fe surface Loval & Yes: & Yes & Yes & Yos & Yes \\
\hline Uenual Tape Surface Lovel & Ho & Ho & Yos & No & Ho \\
\hline EHRAF Gauge & Ho & Ho . & Ho & Ho & Ho \\
\hline Weste Type & DC & OSSF & HCPLX & DSSF & DSSF \\
\hline Total Wasto & $1,730 \mathrm{~kL}(456 \mathrm{~kg}=1)$ & $2,060 \mathrm{~kL}(543 \mathrm{kgal})$ & $2,470 \mathrm{~kL}(652 \mathrm{kgal})$ & $2,320 \mathrm{~kL}(614 \mathrm{~kg} I)$ & $2,590 \mathrm{~kL} \mathrm{(683} \mathrm{kgai)}$ \\
\hline Supernato & $3.78 \mathrm{~kL}$ (1 kgal) & o & $3.70 \mathrm{~kL}$ (1 kgal) & 0 & $\infty$ \\
\hline Dralneble Interetftel Liquld & $840 \mathrm{~kL}$ (145 kgal) & $683 \mathrm{~kL}$ (183 kgat) & $878 \mathrm{~kL}(232 \mathrm{kgal})$ & $761 \mathrm{~kL}(201 \mathrm{kgal})$ & $888 \mathrm{kl}(281 \mathrm{~kg}=1)$ \\
\hline Sludge & 424 KL (112 kgaI) & $443 \mathrm{~kL}$ (117 kge1) & $435 \mathrm{~kL}$ (115 kgal) & $515 \mathrm{~kL}(136 \mathrm{kgal})$ & $276 \mathrm{~kL}$ (73 kgan) \\
\hline Salt Cake & $1,300 \mathrm{~kL}(343 \mathrm{kgal})$ & $1,610 \mathrm{~kL} \mathrm{(426} \mathrm{kgai)}$ & $2,030 \mathrm{~kL}(536 \mathrm{kgal})$ & $1,810 \mathrm{~kL}(478 \mathrm{kgal})$ & $2,310 \mathrm{~kL}(610 \mathrm{kgal})$ \\
\hline Integrity Category & Sound & Sound & Sound & ASMD LKR & Sound \\
\hline Leak Vol. Accum. (laet update) & 0 . & - & 0. & $227 \mathrm{~kL}(6 \mathrm{kgal})$ (1888\} & -0 \\
\hline Wateh Llat Status & Kydrogon & Hydrogen & Hydrogen & Hydrogen & Mydrogon \\
\hline Temporature & $59.4^{\circ} \mathrm{C}\left(139^{\circ} \mathrm{F}\right)$ & $67.2^{\circ} \mathrm{C}\left(153^{\circ} \mathrm{F}\right)$ & $80.6^{\circ} \mathrm{C}\left(177^{\circ} \mathrm{F}\right)$ & $76.7^{\circ} \mathrm{C}\left(170^{\circ} \mathrm{F}\right)$ & $80.0^{\circ} \mathrm{C}\left(176^{\circ} \mathrm{F}\right)$ \\
\hline stabilusolation & IPI & IPI & IPI & IPI & IPI \\
\hline
\end{tabular}

Table 4-20. 241-SX Tank Farm Status. (sheet 2 of 3)

\begin{tabular}{|c|c|c|c|c|c|}
\hline Tink & $241,5 \times 906$ \& & $4,15 x 107 \%$ & $2415 x+09,-1$ & $2415 \times 109-1$ & $24 \leq x+10$ \\
\hline Out of Servlce & 1980 & 1964 & 1962 & 1985 & 1975 \\
\hline $\begin{array}{l}\text { Menford } \\
\text { Coordinates }\end{array}$ & $\begin{array}{l}35,450^{\circ} \mathrm{H} \\
75,869^{\circ} \mathrm{W}\end{array}$ & $\begin{array}{l}35,348^{\circ} \mathrm{K} \\
75,665 \% \mathrm{~W}\end{array}$ & $\begin{array}{l}35,348^{\circ} \mathrm{N} \\
75,767^{\circ} W\end{array}$ & $\begin{array}{l}35,348^{\circ} \mathrm{N} \\
75,868^{\circ} \mathrm{W}\end{array}$ & $\begin{array}{l}35,246^{\circ} \mathrm{W} \\
75,665^{\circ} \mathrm{W}\end{array}$ \\
\hline Rlsers, Total & 12 & 22 & 22 & 21 & 21 \\
\hline FIC Suriace Level & Yes: & No & No & No & No \\
\hline Manual Tape Surface Lovol & YeE & Yes & Yos & Yos & Yes \\
\hline ENRAF Gauge & Yos & No & Ho & No & Ho \\
\hline Waste Typo & NCPLX & NCPLX & HCPLX & KCPLX & HCPLX \\
\hline Totat Waste & $2,040 \mathrm{~kL}(538 \mathrm{kgal})$ & $394 \mathrm{~kL}(104 \mathrm{kgal})$ & $329 \mathrm{~kL}$ (87 kgal) & $846 \mathrm{~kL}(250 \mathrm{~kg}=1)$ & $235 \mathrm{~kL}(62 \mathrm{kgal})$ \\
\hline supernate & $231 \mathrm{~kL}$ (6) kgat) & -0 & -0 & -0 & - \\
\hline Drainable Interstitial Liquid & $734 \mathrm{~kL}$ (184 kgai) & $18.9 \mathrm{~kL}$ (5 kgal) & $18.9 \mathrm{~kL}(5 \mathrm{kgal})$ & $37.9 \mathrm{~kL}(10 \mathrm{~kg} \mathrm{al})$ & -0 \\
\hline sludge & $45.4 \mathrm{~kL}(12 \mathrm{kgal})$ & $384 \mathrm{~kL}(104 \mathrm{~kg} / \mathrm{l})$ & $329 \mathrm{~kL}$ (87 kgal) & -0. & $235 \mathrm{~kL}$ (62 kgal) \\
\hline Salt Cake & $1,760 \mathrm{~kL}$ (485 kgal) & 0. & - & $946 \mathrm{~kL}(250 \mathrm{kgal})$ & - \\
\hline Integrity Catogory & Sound & ASMD LKR & ASHD LKR & ASHD LKR & ASHD LKR \\
\hline $\begin{array}{l}\text { Lonk Vol Accum. } \\
\text { (lest update) }\end{array}$ & $\infty$ & $\begin{array}{l}<18.9 \mathrm{~kL} \\
\text { (<5 kgal) (1983) }\end{array}$ & $\begin{array}{l}90.8 \text { to } 132 \mathrm{~kL} \\
(24 \text { to } 35 \mathrm{kgal}) \text { (1981) }\end{array}$ & $\begin{array}{l}<37.9 \mathrm{~kL} \\
\text { (< } 10 \mathrm{~kg}=\text { (189) }\end{array}$ & $\begin{array}{l}20.8 \mathrm{~kL} \\
(5.5 \mathrm{kgal})(1889)\end{array}$ \\
\hline Watch List Status & Ferrocyenido & Not Listod & Farrocyanide & Ferrocyanide & Not Liated \\
\hline Tomperature & $44.4^{\circ} \mathrm{C}\left(112^{\circ} \mathrm{F}\right)$ & $78.9^{\circ} \mathrm{C}\left(174^{\circ} \mathrm{F}\right)$ & $83.9^{\circ} \mathrm{C}\left(201^{\circ} \mathrm{F}\right)$ & $67.2^{\circ} \mathrm{C}\left(153^{\circ} \mathrm{F}\right)$ & $80.6^{\circ} \mathrm{C}\left(177^{\circ} \mathrm{F}\right)$ \\
\hline Stabilisisolation & IPI & IPI & IPI & IPI & IPI \\
\hline
\end{tabular}




\begin{tabular}{|c|c|c|c|c|c|}
\hline$d \mid / S I$ & $d \mid / S I$ & $d \mathrm{l} / \mathrm{Sl}$ & $d|/ s|$ & $d l / S I$ & uo!̣E|OS!л!qEIS \\
\hline S/O & $\begin{array}{l}(\text { (J.LL) } \\
\text { O.E.88 }\end{array}$ & $\left(H_{0} L L\right) J_{.0}, 9 Z$ & $\begin{array}{l}(-291) \\
0.2 .2 L\end{array}$ & $\left(U_{0} 96 b\right) 0.9 \cdot 06$ & 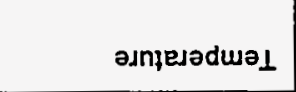 \\
\hline pəISI ION & pəIS! ION & paIS!7 ION & peis!] ION & peIs!7 ION & 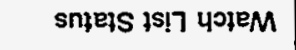 \\
\hline $\begin{array}{r}\{2661\} \\
(\text { (1E6y 05) } \\
7 \times 681\end{array}$ & 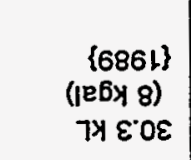 & $\begin{array}{r}\{9861\} \\
(j e 6 y 91) \\
74899\end{array}$ & 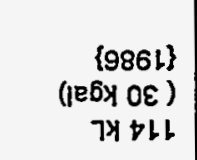 & 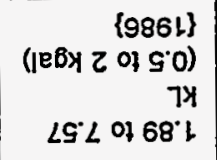 & 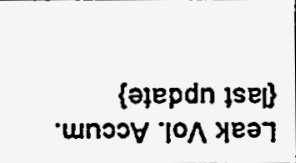 \\
\hline צ׳ㄱ awsy & צหา awS & У즈 aws & ४X7 aWS & પำ1 aWS & 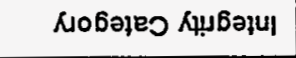 \\
\hline-0 & -0 & -0 & -0 & $-\sigma$ & әXеЈ HES \\
\hline 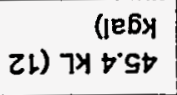 & $\begin{array}{r}(1 \mathrm{e} 6 y \\
\text { L81) } 989\end{array}$ & $\begin{array}{r}\text { (je6y } \\
92) 7 \nabla^{\circ} 86\end{array}$ & $\begin{array}{r}(1 \mathrm{e} 6 x \\
\tau 6) 7 \text { 가 } 8 \nabla \varepsilon\end{array}$ & 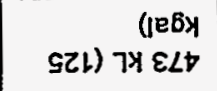 & ә6pnis \\
\hline-0 & 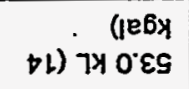 & -0 & 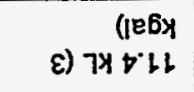 & $\begin{array}{r}\text { (ןeธิx } \\
\text { L) } 7 x 9^{\circ} 92\end{array}$ & 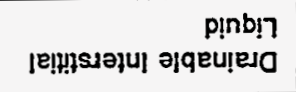 \\
\hline-0 & $-\sigma$ & -0 & $-\infty$ & -0 & ə]euədns \\
\hline $\begin{array}{r}(1 e 6 x \\
z L) 7 x+5 t\end{array}$ & $\begin{array}{r}\text { (je6y } \\
\text { 18々) 거 } 989\end{array}$ & (ןЕБх 9Z) †86 & $\begin{array}{r}\text { (|ЕBप } \\
26) 7 \text { ᄀ } 87 \varepsilon\end{array}$ & $\begin{array}{r}(1 \varepsilon 6 y \\
G Z L) 7 \times \varepsilon \angle t\end{array}$ & әjseM |EjOL \\
\hline X7dON & XTdכN & $X 7 d O N$ & X7dכN & X7dON & ad $K_{\perp}$ ə)seM \\
\hline ON & ON & ON & ON & ON & 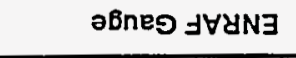 \\
\hline sol & səג & sal & $\operatorname{sa\lambda }$ & sed & $\begin{array}{r}\text { ןə^əך] } \\
\text { aoejuns ade } \perp \text { IEnueW }\end{array}$ \\
\hline ON & ON & oN & ON & ON & 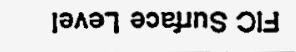 \\
\hline or & 12 & H & 12 & $0 Z$ & |EłOL 'sJəs!y \\
\hline $\begin{array}{l}\text { Mo698'GL } \\
\text { Notbl'SE }\end{array}$ & $\begin{array}{l}\text { MoL9L'SL } \\
\text { NottL'SE }\end{array}$ & $\begin{array}{l}\text { M.S99'SL } \\
\text { NottL'SE }\end{array}$ & $\begin{array}{l}\text { M.698'SL } \\
\text { N.9bZ'GE }\end{array}$ & $\begin{array}{l}\text { MoL9L'SL } \\
\text { No9tZ'SE }\end{array}$ & $\begin{array}{r}\text { sojeulpsoos } \\
\text { plojueH }\end{array}$ \\
\hline S96b & ZL6L & 8961 & 6981 & $\nabla \angle 6$ & 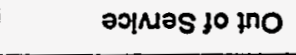 \\
\hline $911 \times 5,172$ & $60 \times 5 \%$ & 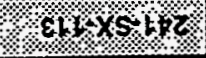 & $2 \% \times 5 \%$ & 1\% $1 \% 5 \%$ & 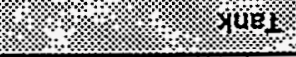 \\
\hline
\end{tabular}

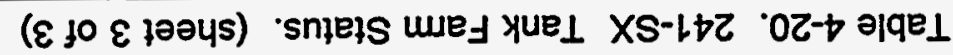




\section{WHC-SD-WM-TI-648 REV 0}

Figure 4-5. Typical Single-Shell Tank Configuration (241-A, $-A X,-S X)$.

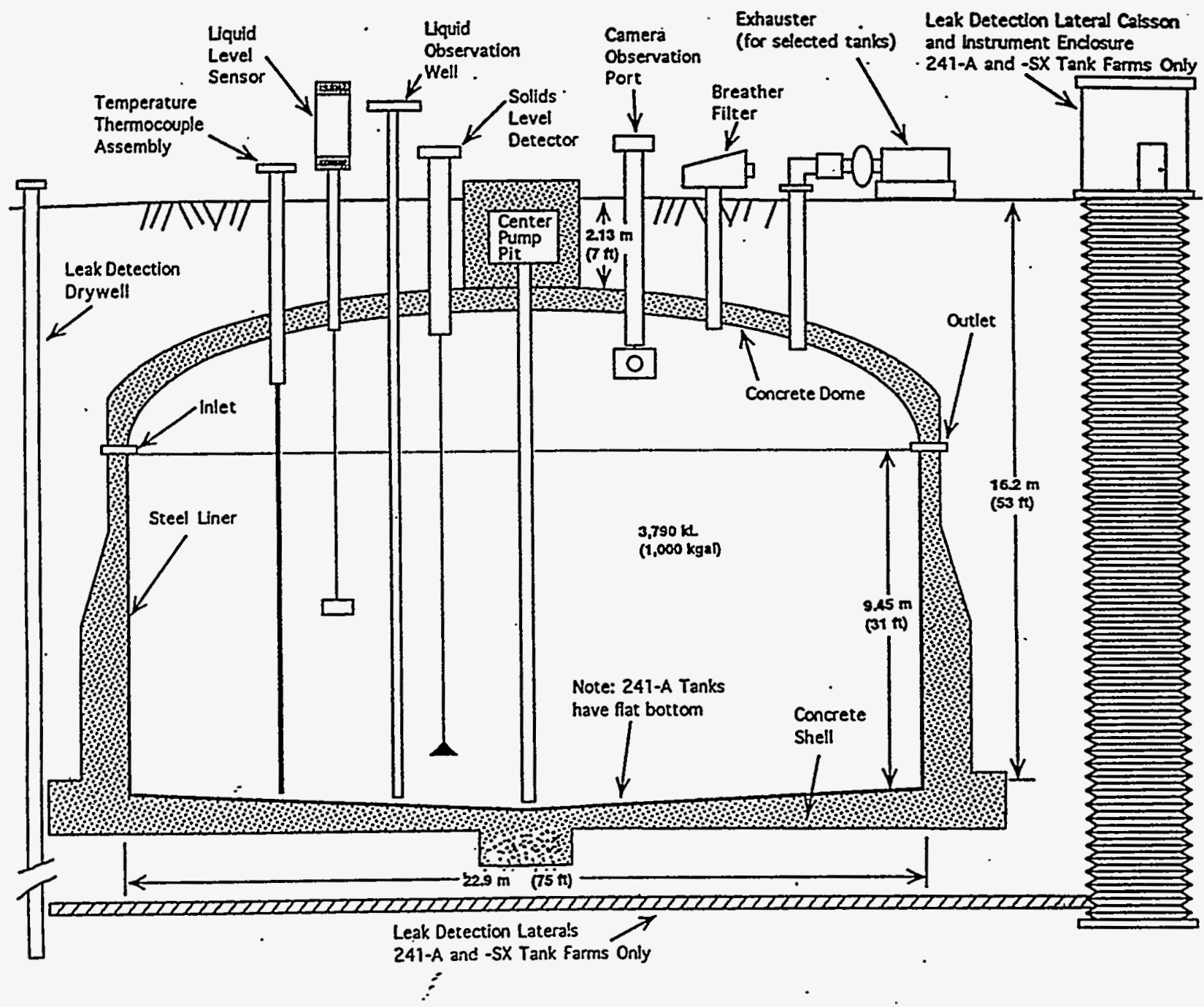




\subsubsection{1-A Tank Farm}

The 241-A Tank Farm is located in the 200 East Area. This six-tank farm was constructed in 1951-52. These tanks are a fourth generation flat dished design with capacities of $3,790 \mathrm{~kL}$ $(1,000 \mathrm{kgal}$ )(see Figure $4-5)$. This design included vitreous clay condenser risers connected to above ground fluid-to-air condensers. All the A Tank Farm tanks were used to store PUREX Plant high-level waste for aging. Tank 241-A-105 received PUREX inorganic wash waste for three years before a violent reaction occurred in 1965 which caused the tank to rupture. The tank was immediately taken out of service and emptied in 1977. In the 1970's, the remaining tanks were sluiced for use as 242-A Evaporator feed and bottoms-receiver tanks. A ventilation exhauster was installed on Tank 241-A-105. An underground vent header connects all six tanks to provide airflow to the other five tanks.

Table 4-21 provides additional information about the A Tank Farm. Table 4-22 provides status of the individual tanks in the A Tank Farm, including current stabilization status as of March 1994 (Hanlon).

Table 4-21. 241-A Tank Farm Generic Description.

\begin{tabular}{|l|l|}
\hline Type & Single-Shell \\
\hline Constructed & $1954-55$ \\
\hline In Service & $1956:(1959:-104,1962:-105,1957:-106)$ \\
\hline Diameter & $22.9 \mathrm{~m}(75 \mathrm{ft})$ \\
\hline Maximum Waste Depth & $9.24 \mathrm{~m}(30.3 \mathrm{ft})$ \\
\hline Operating Capacity & $3,790,000 \mathrm{~L}(1,000$ kgal) \\
\hline Bottom Shape & Flat \\
\hline Ventilation & Operating Exhauster (Active) \\
\hline $\begin{array}{l}\text { External Drywells (each } \\
\text { tank) }\end{array}$ & $7(10:-101)$ \\
\hline Lateral Wells & 3 \\
\hline Airlift Circulators (each) & 4 \\
\hline
\end{tabular}


Table 4-22. 241-A Tank Farm Status.

\begin{tabular}{|c|c|c|c|c|c|c|}
\hline rank & $2417104 \%$ & $241 \% 102 \%$ & $441 \times-103$ & $4419-104$ & $241 \times 105 \%$ & $241+106$ \\
\hline Out of Service & 1980 & 1980 & 1980 & 1975 & 1963 & 1980 \\
\hline $\begin{array}{l}\text { Hanford } \\
\text { Coordinates }\end{array}$ & $\begin{array}{l}41,205^{\circ} \mathrm{N} \\
47,804^{\circ} \mathrm{W}\end{array}$ & $\begin{array}{l}41,205^{\circ} \mathrm{N} \\
47,702^{\circ} \mathrm{W}\end{array}$ & $\begin{array}{l}41,205^{\circ} \mathrm{N} \\
47,600^{\circ} \mathrm{W}\end{array}$ & $\begin{array}{l}41,307^{\circ} \mathrm{N} \\
47,804^{\circ} \mathrm{W}\end{array}$ & $\begin{array}{l}41,307^{\circ} \mathrm{N} \\
47,702^{\circ} \mathrm{W}\end{array}$ & $\begin{array}{l}41,307^{\circ} \mathrm{N} \\
47,600^{\circ} \mathrm{W}\end{array}$ \\
\hline Risers, Total & 22 & 20 & 19 & 21 & 25 & 21 \\
\hline FIC Surface Level & No & Yes & Yes & No & No & Yes \\
\hline $\begin{array}{l}\text { Manual Tape Surface } \\
\text { Level }\end{array}$ & Yes & No & No & Yes & Yes & No \\
\hline ENRAF Gauge & No & No & No & No & No & No \\
\hline Waste Type & DSSF & DSSF & DSSF & NCPLX & NCPLX & CP \\
\hline Total Waste & $\begin{array}{l}3,610 \mathrm{~kL}(953 \\
\mathrm{kgal})\end{array}$ & $\begin{array}{l}155 \mathrm{~kL}(41 \\
\mathrm{kgal})\end{array}$ & $\begin{array}{l}1,400 \mathrm{~kL}(371 \\
\text { kgal) }\end{array}$ & $\begin{array}{l}106 \mathrm{~kL}(28 \\
\mathrm{kgal})\end{array}$ & $\begin{array}{l}71.9 \mathrm{~kL} \mathrm{(19} \\
\mathrm{kgal})\end{array}$ & $\begin{array}{l}473 \mathrm{~kL}(125 \\
\mathrm{kgal})\end{array}$ \\
\hline Supernate & -0 & $\begin{array}{l}15.1 \mathrm{~kL} \mathrm{(4} \\
\mathrm{kgal})\end{array}$ & $\begin{array}{l}18.9 \mathrm{~kL} \mathrm{(5} \\
\mathrm{kgal})\end{array}$ & -0 & -0 & -0 \\
\hline $\begin{array}{l}\text { Drainable Interstitial } \\
\text { Liquild }\end{array}$ & $\begin{array}{l}1,560 \mathrm{~kL} \mathrm{(413} \\
\mathrm{kgal})\end{array}$ & $\begin{array}{l}7.57 \mathrm{~kL}(2 \\
\mathrm{kgal})\end{array}$ & $\begin{array}{l}56.8 \mathrm{~kL} \mathrm{(15} \\
\mathrm{kgal})\end{array}$ & -0 & $\begin{array}{l}15.1 \mathrm{~kL}(4 \\
\mathrm{kgal})\end{array}$ & $\begin{array}{l}26.5 \mathrm{~kL}(7 \\
\mathrm{kgal})\end{array}$ \\
\hline Sludge & $\begin{array}{l}11.4 \mathrm{~kL} \mathrm{(3} \\
\mathrm{kgal})\end{array}$ & $\begin{array}{l}56.8 \text { kL (15 } \\
\text { kgal) }\end{array}$ & $\begin{array}{l}1,390 \mathrm{~kL}(366 \\
\mathrm{kgal})\end{array}$ & $\begin{array}{l}106 \mathrm{~kL}(28 \\
\text { kgal) }\end{array}$ & $\begin{array}{l}71.9 \mathrm{~kL}(19 \\
\text { kgal) }\end{array}$ & $\begin{array}{l}473 \mathrm{~kL}(125 \\
\text { kgal) }\end{array}$ \\
\hline Salt Cake & $\begin{array}{l}3,600 \mathrm{~kL}(950 \\
\text { kgal) }\end{array}$ & $\begin{array}{l}83.3 \mathrm{~kL} \mathrm{(22} \\
\text { kgal) }\end{array}$ & -0 & -0 & -0 & -0 \\
\hline Integrity Category & Sound & Sound & ASMD LKR & ASMD LKR & ASMD LKR & Sound \\
\hline $\begin{array}{l}\text { Leak Vol. Accum. } \\
\text { \{last update\} }\end{array}$ & -0 & -0 & $\begin{array}{l}1.89 \text { to } 20.8 \mathrm{~kL} \\
(0.5 \text { to } 5.5 \\
\mathrm{kgal}) \\
\{1987\}\end{array}$ & $\begin{array}{l}9.46 \mathrm{~kL} \\
(2.5 \mathrm{kgal}) \\
\{1983\}\end{array}$ & $\begin{array}{l}37.9 \text { to } 1,050 \\
\mathrm{~kL} \\
\text { (10 to } 277 \\
\mathrm{kgal})\{1991\}\end{array}$ & -0 \\
\hline Watch List Status & Hydrogen & Not Listed & Not Listed & Not Listed & Not Listed & Not Listed \\
\hline $\begin{array}{c}\text { Temperature } \\
=\end{array}$ & $67.2^{\circ} \mathrm{C}\left(153^{\circ} \mathrm{F}\right)$ & $30.6^{\circ} \mathrm{C}\left(87^{\circ} \mathrm{F}\right)$ & $45.6^{\circ} \mathrm{C}\left(114^{\circ} \mathrm{F}\right)$ & $\begin{array}{l}88.3^{\circ} \mathrm{C} \\
\left(191^{\circ} \mathrm{F}\right)\end{array}$ & $\begin{array}{l}60.0^{\circ} \mathrm{C} \\
\left(140^{\circ} \mathrm{F}\right)\end{array}$ & $\begin{array}{l}57.2^{\circ} \mathrm{C} \\
\left(135^{\circ} \mathrm{F}\right)\end{array}$ \\
\hline Stabil//solation & IPI & IS/PI & IS/IP & IS/IP & IS $/ \mathrm{PP}$ & ISAP \\
\hline
\end{tabular}




\subsubsection{1-AX Tank Farm}

The 241-AX Tank Farm, located in the 200 East Area, is the last group of SSTs built at the Hanford Site. Built in 1963-64, this four-tank farm uses a similar design as the 241-A Tank Farm (see Figure 4-5). The design incorporates a grid of drainage slots beneath the steel liner to provide observation and collection of potential tank leakage. The riser pattern was altered into a unique pattern to enhance waste agitation with 22 airlift circulators. The AX Tank Farm was designed to accept self-boiling waste and was used to store $B$ Plant high-level waste and PUREX Plant organic and inorganic wash waste. In the 1970's Tanks 241-AX-101 through -AX-103 were sluiced and used for evaporator slumy receiver tanks. Tank 241-AX-104 was removed from service due to questionable integrity.

Table 4-23 describes additional information pertaining to the AX Tank Farm. Table 4-24 provides status of the individual tanks, including current stabilization status as of March 1994 (Hanlon).

Table 4-23. 241-AX Tank Farm Generic Description.

\begin{tabular}{|l|l|}
\hline Type & Snghestill \\
\hline Constructed & $1963-64$ \\
\hline In Service & $1965:-101,-103,1966:-102,-104)$ \\
\hline Out of Service & $1980(1974:-104)$ \\
\hline Diameter & $22.9 \mathrm{~m}(75 \mathrm{ft})$ \\
\hline Maximum Waste Depth & $9.24 \mathrm{~m}(30.3 \mathrm{ft})$ \\
\hline Operating Capacity & $3,790,000 \mathrm{~L}(1,000 \mathrm{kgal})$ \\
\hline Bottom Shape & Flat \\
\hline Ventilation & Passive \\
\hline $\begin{array}{l}\text { External Drywells (each } \\
\text { tank) }\end{array}$ & $8:-101,10:-102,6:-103,7:-104$ \\
\hline Lateral Wells & 0 \\
\hline
\end{tabular}


Table 4-24. 241-AX Tank Farm Status.

\begin{tabular}{|c|c|c|c|c|}
\hline Tank & $241+0 \times 101$ & $241+4 x-102$ & $241+A x-103$ & $241-A x-104$ \\
\hline $\begin{array}{l}\text { Hanford } \\
\text { Coordinates }\end{array}$ & $\begin{array}{l}41,731^{\circ} \mathrm{N} \\
47,475^{\circ} \mathrm{W}\end{array}$ & $\begin{array}{l}41,621^{\circ} \mathrm{N} \\
47,475^{\circ} \mathrm{W}\end{array}$ & $\begin{array}{l}41,731^{\circ} \mathrm{N} \\
47,565^{\circ} \mathrm{W}\end{array}$ & $\begin{array}{l}41,621^{\circ} \mathrm{N} \\
47,565^{\circ} \mathrm{W}\end{array}$ \\
\hline Risers, Total & 37 & 36 & 35 & 38 \\
\hline FIC Surface Level & Yes & No & Yes & No \\
\hline $\begin{array}{l}\text { Manual Tape Surface } \\
\text { Level }\end{array}$ & No & Yes & No & Yes \\
\hline ENRAF Gauge & No & No & No & No \\
\hline Waste Type & DSSF & $\mathrm{CC}$ & cC & NCPLX \\
\hline Total Waste & $\begin{array}{l}2,830 \mathrm{~kL}(748 \\
\mathrm{kgal})\end{array}$ & $\begin{array}{l}148 \mathrm{~kL}(39 \\
\mathrm{kgal})\end{array}$ & $\begin{array}{l}424 \mathrm{~kL}(112 \\
\mathrm{kgal})\end{array}$ & $\begin{array}{l}26.5 \mathrm{~kL}(7 \\
\mathrm{kgal})\end{array}$ \\
\hline Supernate & -0 & $\begin{array}{l}11.4 \mathrm{~kL}(3 \\
\mathrm{kgal})\end{array}$ & -0 & -0 \\
\hline $\begin{array}{l}\text { Drainable Interstitial } \\
\text { Liquid }\end{array}$ & $\begin{array}{l}1,210 \mathrm{~kL}(320 \\
\mathrm{kgal})\end{array}$ & $\begin{array}{l}53.0 \mathrm{~kL}(14 \\
\mathrm{kgal})\end{array}$ & $\begin{array}{l}136 \mathrm{~kL}(36 \\
\mathrm{kgal})\end{array}$ & -0 \\
\hline Sludge & $\begin{array}{l}11.4 \mathrm{~kL} \mathrm{(3} \\
\mathrm{kgal})\end{array}$ & $\begin{array}{l}26.5 \mathrm{~kL}(7 \\
\mathrm{kgal})\end{array}$ & $\begin{array}{l}7.57 \mathrm{~kL}(2 \\
\text { kgal) }\end{array}$ & $\begin{array}{l}26.5 \mathrm{~kL}(7 \\
\mathrm{kgal})\end{array}$ \\
\hline Salt Cake & $\begin{array}{l}2,820 \mathrm{~kL}(745 \\
\mathrm{kgal})\end{array}$ & $\begin{array}{l}110 \mathrm{~kL}(29 \\
\text { kgal) }\end{array}$ & $\begin{array}{l}416 \mathrm{~kL}(110 \\
\mathrm{kgal})\end{array}$ & -0 \\
\hline Integrity Category & Sound & ASMD LKR & Sound & ASMD LKR \\
\hline $\begin{array}{l}\text { Leak Vol. Accum. } \\
\text { \{last update\} }\end{array}$ & $-0-$ & $\begin{array}{l}11.4 \text { kL (3 } \\
\text { kgal) } \\
\{1989\}\end{array}$ & $-0-$ & $\begin{array}{l}30.3 \mathrm{~kL}(8 \\
\mathrm{kgal}) \\
\{1989\}\end{array}$ \\
\hline Watch List Status & Hydrogen & Not Listed & Hydrogen & Not Listed \\
\hline Temperature & $57.8^{\circ} \mathrm{C}\left(136^{\circ} \mathrm{F}\right)$ & $21.7^{\circ} \mathrm{C}\left(71^{\circ} \mathrm{F}\right)$ & $\begin{array}{l}47.2^{\circ} \mathrm{C} \\
\left(117^{\circ} \mathrm{F}\right)\end{array}$ & $33.3^{\circ} \mathrm{C}\left(92^{\circ} \mathrm{F}\right)$ \\
\hline Stabil/lsolation & IPI & ISIP & IS/P & IS/P \\
\hline
\end{tabular}




\subsection{DOUBLE-SHELL TANK BACKGROUND}

The text based information provided in this section that describes double-shell tanks has been obtained from Koerna (1986), Winters et al. (1990), and Koontz (1989). Tabular Information was acquired from Hanlon (1994), Husa et al. (1993), and Alstad (1992). Figure 4-6 was generated from information obtained from Hanlon (1994) and Anderson (1990).

Twenty-eight double-shell tanks (DSTs) were constructed in the 200 Areas to provide intermediate storage for aging and nonaging, high-level radioactive waste, including Hanford facility waste, evaporator feed, and slurry. Table 4-25 describes the double-shell tank farms. All the DSTs are similar in design and construction. However, the tanks and ancillary equipment can differ slightly because of design improvements and variations in waste composition. The double-shell design (see Figure 4-6) minimizes the potential for leakage of radioactive liquids to the environment. Each tank is constructed of two steel liners within a protective shell. The protective shell is constructed of reinforced concrete designed to sustain soil loads and temperature gradients generated by the contained radioactive waste. The inner wall of the concrete shell is lined with a carbon-steel liner referred to as the secondary steel tank. The primary tank, also constructed of carbon-steel, is completely enclosed within the secondary tank.

Table 4-25. Double-Shell Tank Farm Generic Description.

\begin{tabular}{|l|l|}
\hline Type & Dofbleshetl \\
\hline Constructed & $1970-86$ \\
\hline In Service & $1971-86$ \\
\hline Diameter & $22.9 \mathrm{~m}(75 \mathrm{ft})$ \\
\hline Maximum Waste Depth & $10.7 \mathrm{~m}$ (35 ft) AYIAZ: 9.27m (30.4 ft) \\
\hline Operating Capacity & $\begin{array}{l}4,320,000 \mathrm{~L}(1,140 \mathrm{kgal}) \\
(1,000 \mathrm{kgal})\end{array}$ \\
\hline Bottom Shape & AY/AZ: 3,790,000 L \\
\hline Ventilation & Operating Exhauster (Active) \\
\hline Air lift circulators (each) & AY (22), AZ (22), AN-107(21), SY-102, AW-102 \\
\hline Risers, total & $\begin{array}{l}59: \text { AN (61: AN-101, 80: AN-107) } \\
71: \text { AP (72: AP-102) } \\
59: \text { AW } \\
\text { 58: SY (59: SY-102) }\end{array}$ \\
\hline
\end{tabular}

The primary tank is freestanding and is positioned on an insulated concrete pad. The insulated concrete pad protected the structural concrete foundation from excessive temperatures during annealing or stress relief treatment of the primary tank. Annealing reduces high stress points at metal joints to reduce the susceptibility of corrosion and cracking. The insulating pad is also cast with air distribution and drain grids to provide for leak detection, maintain a uniform tank bottom temperature, facilitate heat removal, and eliminate pockets of water condensation. An annular 
space separates the two steel liners and serves as a containment barrier for primary tank leakage. The tanks are actively vented (with fan-driven ventilation systems) to the environment. The primary ventilation system removes vapors from the primary tank. The annulus ventilation system is used to cool the primary tank, remove moisture accumulation in the annular space, and aid in detection of radioactive leakage. Leak detection and monitoring systems consist of: (1) annulus leak detection, (2) tank sludge level measurement, (3) tank pressure and temperature measurement, (4) tank liquid level measurement, (5) area radiation (gamma) detection, (6) process pipeline heat tracing, (7) exhaust stack surveillance, and (8) process pipeline and pit leak detection. Ancillary equipment that support the tank farms include waste transfer pipelines, valves, diversion boxes, jumper connectors, pumps, catch stations, catch tanks, and process pits (WHC, 1993). The six double-shell tank farms, consisting of two to eight tanks each, are described in the following sections. 
Figure 4-6. Typical Double-Shell Tank Configuration.

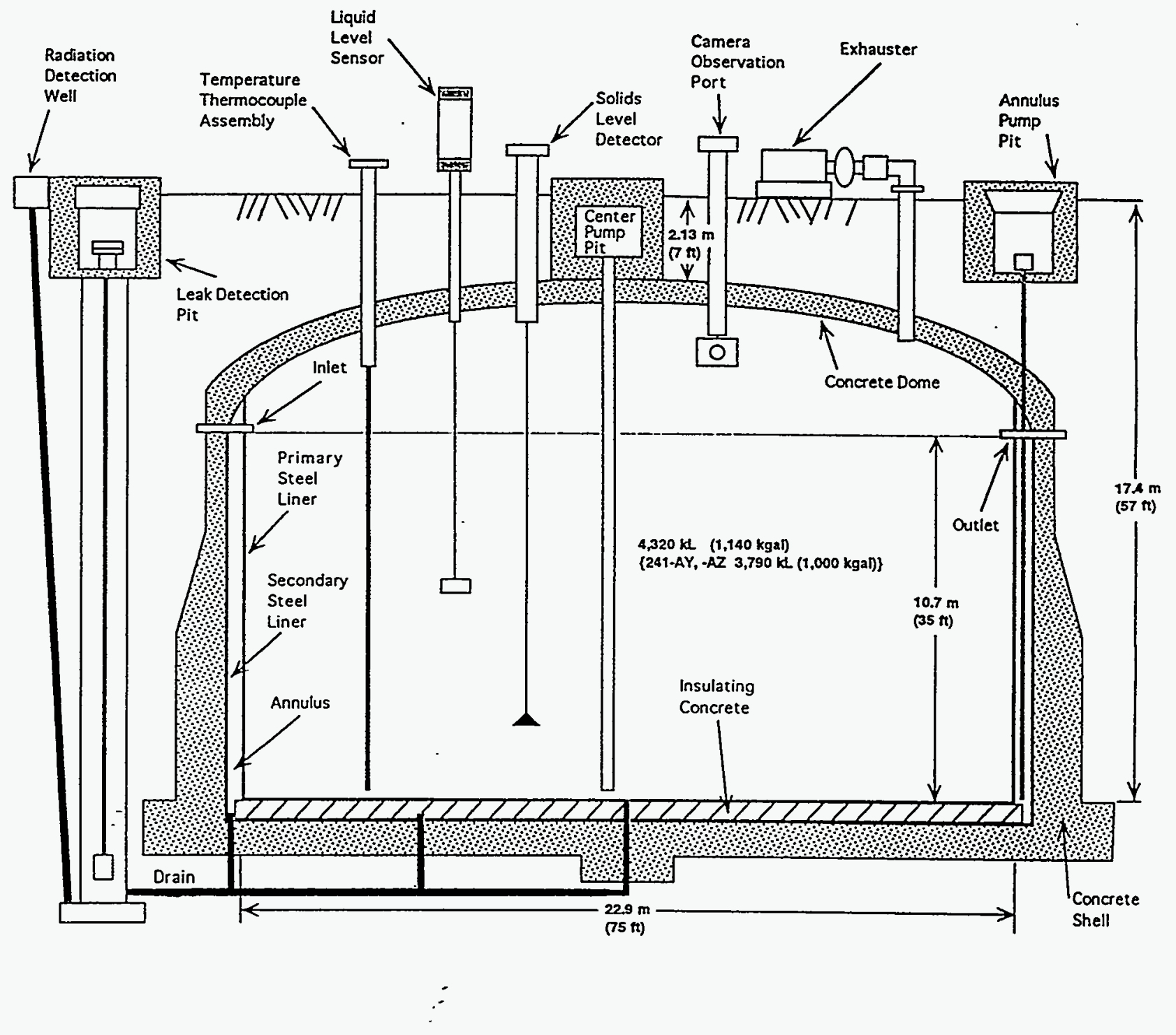




\subsubsection{1-AP Tank Fam}

The 241-AP Tank Farm, in the 200 East Area, consists of eight tanks. These double-shell tanks have received dilute noncomplexed waste from PUREX and other operations, and also slurry feed from the 242-A Evaporator. Liquid wastes are transferred to and from the AP Tank Farm through the AW Tank Farm facility. Tank AP-102 contains a mixing pump and was used to feed waste to the Hanford Grout Facility. Table 4-26 provides status of the individual tanks as of March 1994 (Hanlon).

Table 4-26. 241-AP Tank Farm Status.

\begin{tabular}{|c|c|c|c|c|}
\hline Tank & 21 ap 101 औ & 241 Ap 102,04 & 24 ophos & $2 x y+104$ : \\
\hline $\begin{array}{l}\text { Henford } \\
\text { Coordinates }\end{array}$ & $\begin{array}{l}40,688 \cdot \mathrm{W} \\
47,100 \% \mathrm{~W}\end{array}$ & $\begin{array}{l}40,666^{\circ} \mathrm{N} \\
48,868^{\circ} \mathrm{W}\end{array}$ & $\begin{array}{l}40,559 \cdot \mathrm{N} \\
\mathrm{dT,100 \%}\end{array}$ & $\begin{array}{l}40,559^{\circ} \mathrm{W} \\
46,968^{\circ} \mathrm{W}\end{array}$ \\
\hline FIC Surfaco Lovel & $978 \mathrm{~cm}(385 \mathrm{in})$ & N/A & $1,040 \mathrm{~cm}(411 \mathrm{ln})$ & $16.5 \mathrm{~cm}(6.5 \mathrm{ln})$ \\
\hline Manual Tapo Surface Level & $878 \mathrm{~cm}(385 \mathrm{in})$ & $1,020 \mathrm{~cm}(401 \mathrm{~h})$ & $1,040 \mathrm{~cm}(411 \mathrm{ln})$ & N/A \\
\hline ENRAF Gauge & Ho & Ho & No & No \\
\hline Wasto Typo & DH & DN & DH & DN \\
\hline Total Waste & $4,010 \mathrm{~kL}(1,060 \mathrm{~kg} /)$ & $4,160 \mathrm{kd}(1,100 \mathrm{kgal})$ & $4,280 \mathrm{~kL}(1,130 \mathrm{kgal})$ & $68.1 \mathrm{~kL}(18 \mathrm{~kg} 21)$ \\
\hline Supernate & $4,010 \mathrm{~kL}(1,060 \mathrm{~kg}=1)$ & $4,160 \mathrm{~kL}(1,100 \mathrm{kgal})$ & $4,280 \mathrm{HL}(1,130 \mathrm{kgal})$ & $68.1 \mathrm{~kL}$ (18 kgal) \\
\hline Drainable Intoratitial Liquid & $\infty$ & -0 & $\infty$ & -0 \\
\hline sludge & -0 & - & - & - \\
\hline Integrity Catogory & Sound & Sound & Sound & Sound \\
\hline Watch Litet Status & Not Liztod & Not Lited & Not Liztod & Not Lieted \\
\hline Tomperature & $15.0^{\circ} \mathrm{C}\left(59^{\circ} \mathrm{F}\right)$ & $27.8^{\circ} \mathrm{C}\left(82^{\circ} \mathrm{F}\right)$ & $15.6^{\circ} \mathrm{C}\left(60^{\circ} \mathrm{F}\right)$ & $14.4^{\circ} \mathrm{C}\left(58^{\circ} \mathrm{F}\right)$ \\
\hline Tank Ueo & DRCVR & Grout Feod & DRCVR & Grout Food \\
\hline
\end{tabular}

Table 4-26. 241-AP Tank Farm Status (Continued).

\begin{tabular}{|c|c|c|c|c|}
\hline Iank & 241 AP $105, \ldots$ & $24+4106$ & $241+40-107$ A & $241+4 p+108$ \\
\hline $\begin{array}{l}\text { Hanford } \\
\text { Coordinates }\end{array}$ & $\begin{array}{l}40,393 \% \mathrm{~N} \\
47,100 \mathrm{~W}\end{array}$ & $\begin{array}{l}40,393^{\circ} \mathrm{N} \\
46,968^{\circ} \mathrm{W}\end{array}$ & $\begin{array}{l}40,286 \% \\
47,100 \%\end{array}$ & $\begin{array}{l}40,286^{\circ} \mathrm{N} \\
46,968^{\circ} \mathrm{W}\end{array}$ \\
\hline FIC Surface Level & $757 \mathrm{~cm}(298 \mathrm{in})$ & $1,040 \mathrm{~cm}(410 \mathrm{in})$ & $1,030 \mathrm{~cm}(404 \mathrm{in})$ & $831 \mathrm{~cm}$ (327 in) \\
\hline Manua! Tape Surface Level & NA & NA & N/A & NA \\
\hline ENRAF Gauge & No & No & No & No \\
\hline Waste Type & DSșf́ & DN & DN & DN \\
\hline Total Waste & $3,100 \mathrm{~kL}(820 \mathrm{kgal})$ & $4,280 \mathrm{~kL}(1,130 \mathrm{kgal})$ & $4,200 \mathrm{KL}(1,110 \mathrm{kgal})$ & $3,400 \mathrm{~kL}$ (898 kgal) \\
\hline Supemate & $3,100 \mathrm{KL}(820 \mathrm{kga})$ & $4,280 \mathrm{k} \perp(1,130 \mathrm{kgal})$ & $4,200 \mathrm{KL}(1.110 \mathrm{kgal})$ & $3,400 \mathrm{~K}-(898 \mathrm{kgal})$ \\
\hline Drainable Interstitial Liquid & -0 & -0 & -0 & 0 \\
\hline Siudge & $-a$ & -0 & -0 & -0 \\
\hline Integrity Category & Sound & Sound & Sound & Sound \\
\hline Watch List Status & Not Listed & Nat Listed & Not Listed & Not Listed \\
\hline Temperature & $22.2^{\circ} \mathrm{C}\left(72^{\circ} \mathrm{A}\right)$ & $20.0^{\circ} \mathrm{C}\left(68^{\circ} \mathrm{P}\right)$ & $14.4^{\circ} \mathrm{C}\left(58^{\circ} \mathrm{F}\right)$ & $18.9^{\circ} \mathrm{C}\left(66^{\circ} \mathrm{F}\right)$ \\
\hline Tank Use & CWHT & DRCVR & DRCVR & DRCVR \\
\hline
\end{tabular}




\subsubsection{1-AN Tank Farm}

The 241-AN Tank Farm consists of seven tanks located in the 200 East Area. These tanks were designed for storing 242-A Evaporator Feed and Slurry bottoms (Welty, 1988). Tank 241-AN-107 is equipped with airlift circulators and had the dual capacity to serve as a PUREX high-heat aging-waste receiver. Table 4-27 provides status of the individual tanks, including current stabilization status as of March 1994 (Hanlon).

Table 4-27. 241-AN Tank Farm Status.

\begin{tabular}{|c|c|c|c|c|c|c|c|}
\hline Tank $\quad \because \quad \because$ & 241 ANA & $\begin{array}{l}241+A N \\
102 \%\end{array}$ & $241-A N 103$ & 244 AN & $\begin{array}{l}241-A N= \\
105\end{array}$ & $\begin{array}{l}241-A N-\cdots \\
106\end{array}$ & $\begin{array}{l}\text { 241-AN- } \\
107\end{array}$ \\
\hline $\begin{array}{l}\text { Hanford } \\
\text { Coordinates }\end{array}$ & $\begin{array}{l}42,339.5^{\circ} \\
N \\
47,506^{\circ} \mathrm{W}\end{array}$ & $\begin{array}{l}42,339.5^{\circ} \\
N \\
47,613^{\circ} \\
W\end{array}$ & $\begin{array}{l}42,339.5^{\circ} \mathrm{N} \\
47,720^{\circ} \mathrm{W}\end{array}$ & $\begin{array}{l}42,446.5^{\circ} \mathrm{N} \\
47,506^{\circ} \mathrm{W}\end{array}$ & $\begin{array}{l}40,446.5^{\circ} \\
N \\
47,613^{\circ} \mathrm{W}\end{array}$ & $\begin{array}{l}42,553.5^{\circ} \\
N \\
47,720^{\circ} \\
W\end{array}$ & $\begin{array}{l}42,446.5 \\
{ }^{\circ} \mathrm{N} \\
47,720^{\circ} \\
\mathrm{W}\end{array}$ \\
\hline FIC Surface Level & $\begin{array}{l}665 \mathrm{~cm} \\
(262 \mathrm{in})\end{array}$ & $\begin{array}{l}996 \mathrm{~cm} \\
(392 \mathrm{in})\end{array}$ & $\begin{array}{l}879 \mathrm{~cm} \\
(346 \mathrm{in})\end{array}$ & $\begin{array}{l}978 \mathrm{~cm} \\
\text { (385 in) }\end{array}$ & $\begin{array}{l}1,040 \mathrm{~cm} \\
(411 \mathrm{in})\end{array}$ & $\begin{array}{l}19.3 \mathrm{~cm} \\
(7.6 \mathrm{in})\end{array}$ & N/A \\
\hline $\begin{array}{l}\text { Manual Tape } \\
\text { Surface Level }\end{array}$ & N/A & N/A & N/A & N/A & N/A & N/A & $\begin{array}{l}983 \mathrm{~cm} \\
(387 \mathrm{in})\end{array}$ \\
\hline ENRAF Gauge & No & No & No & No & No & No & No \\
\hline Waste Type & DN & $\mathrm{cc}$ & DSS & DSSF & DSSF & $\mathrm{CP}$ & $\mathrm{CC}$ \\
\hline Total Waste & $\begin{array}{l}2,720 \mathrm{~kL} \\
(719 \mathrm{kgal})\end{array}$ & $\begin{array}{l}4,130 \mathrm{~kL} \\
(1,090 \\
\mathrm{kgal})\end{array}$ & $\begin{array}{l}3,600 \mathrm{~kL} \\
\text { (952 kgal) }\end{array}$ & $\begin{array}{l}4,010 \mathrm{~kL} \\
(1,060 \\
\mathrm{kgal})\end{array}$ & $\begin{array}{l}4,280 \mathrm{~kL} \\
(1,130 \\
\mathrm{kgal})\end{array}$ & $\begin{array}{l}79.5 \mathrm{~kL} \\
(21 \mathrm{kgal})\end{array}$ & $\begin{array}{l}4,050 \mathrm{~kL} \\
(1,070 \\
\mathrm{kgal})\end{array}$ \\
\hline Supernate & $\begin{array}{l}2,720 \mathrm{~kL} \\
(719 \mathrm{kgal})\end{array}$ & $\begin{array}{l}3,790 \mathrm{~kL} \\
(1,000 \\
\text { kgal })\end{array}$ & $\begin{array}{l}56.8 \mathrm{~kL} \\
(15 \mathrm{kgal})\end{array}$ & $\begin{array}{l}3,010 \mathrm{~kL} \\
(794 \mathrm{kgal})\end{array}$ & $\begin{array}{l}4,280 \mathrm{~kL} \\
(1,130 \\
\mathrm{kgal})\end{array}$ & $\begin{array}{l}15.1 \mathrm{~kL} \\
(4 \mathrm{kgal})\end{array}$ & $\begin{array}{l}3,520 \mathrm{~kL} \\
(931 \\
\mathrm{kgal})\end{array}$ \\
\hline $\begin{array}{l}\text { Drainable Interstitial } \\
\text { Liquid }\end{array}$ & -0 & $\begin{array}{l}11.4 \mathrm{~kL} \\
(3 \mathrm{kgal})\end{array}$ & $-0-$ & $\begin{array}{l}94.6 \mathrm{~kL} \\
\text { (25 kgal) }\end{array}$ & -0 & -0 - & $\begin{array}{l}34.1 \mathrm{~kL} \\
(9 \mathrm{kgal})\end{array}$ \\
\hline Sludge & -0 & $\begin{array}{l}337 \mathrm{~kL} \\
(89 \mathrm{kgal})\end{array}$ & $\begin{array}{l}3,550 \mathrm{~kL} \\
\text { (937 kgal) } \\
\text { DSS }\end{array}$ & $\begin{array}{l}999 \mathrm{~kL} \\
\text { (264 kgal) }\end{array}$ & -0 & $\begin{array}{l}64.4 \mathrm{~kL} \\
(17 \mathrm{kgal})\end{array}$ & $\begin{array}{l}507 \mathrm{~kL} \\
(134 \\
\mathrm{kgal})\end{array}$ \\
\hline Integrity Category & Sound & Sound & Sound & Sound & Sound & Sound & Sound \\
\hline Watch List Status & Not Listed & $\begin{array}{l}\text { Not } \\
\text { Listed }\end{array}$ & Hydrogen & Hydrogen & Hydrogen & $\begin{array}{l}\text { Not } \\
\text { Listed }\end{array}$ & $\begin{array}{l}\text { Not } \\
\text { Listed }\end{array}$ \\
\hline Temperature & $\begin{array}{l}21.1^{\circ} \mathrm{C} \\
\left(70^{\circ} \mathrm{F}\right)\end{array}$ & $\begin{array}{l}33.9^{\circ} \mathrm{C} \\
\left(93^{\circ} \mathrm{F}\right)\end{array}$ & $\begin{array}{l}48.3^{\circ} \mathrm{C} \\
\left(119^{\circ} \mathrm{F}\right)\end{array}$ & $\begin{array}{l}49.4^{\circ} \mathrm{C} \\
\left(121^{\circ} \mathrm{F}\right)\end{array}$ & $\begin{array}{l}46.7^{\circ} \mathrm{C} \\
\left(116^{\circ} \mathrm{F}\right)\end{array}$ & $\begin{array}{l}20.0^{\circ} \mathrm{C} \\
\left(68^{\circ} \mathrm{F}\right)\end{array}$ & $\begin{array}{l}35.0^{\circ} \mathrm{C} \\
\left(95^{\circ} \mathrm{F}\right)\end{array}$ \\
\hline Tank Use & DRCVR & CWHT & CWHT & CWHT & CWHT & CWHT & CWHT \\
\hline
\end{tabular}




\subsubsection{1-AW Tank Farm}

The 241-AW Tank Farm consists of six tanks located in the 200 East Area. These tanks were designed for storing 242-A Evaporator Feed and slurry bottoms (Welty, 1988). Tank 241AW-102 is the feed tank for the 242-A-Evaporator; Tank AW-106 is the receiver. To provide a uniform feed to the 242-A Evaporator, Tank AW-102 has airlift circulators for mixing the tank contents and a dip tube assembly to read specific gravity. Additionally, Tank AW-102 has a feed pump pit and drain pit (WHC, 1993). The remaining tanks stored low-heat radioactive liquid waste generated by PUREX. Cladding removal waste was initially stored in AW-103 and AW-105. Solvent extraction wash waste and miscellaneous sump waste was initially stored in AW-104. Table 4-28 provides status of the individual tanks, including current stabilization status as of March 1994 (Hanlon).

Table 4-28. 241-AW Tank Farm Status.

\begin{tabular}{|c|c|c|c|c|c|c|}
\hline rank & 241 AW 101 & 241 aw 102 & 241 AW-103 & $241 A A-104$ & $241-A W-105$ & $241 \mathrm{AW}-106$ \\
\hline $\begin{array}{l}\text { Hanford } \\
\text { Coordinates }\end{array}$ & $\begin{array}{l}40,704^{\circ} \mathrm{N} \\
47,752^{\circ} \mathrm{W}\end{array}$ & $\begin{array}{l}40,704^{\circ} \mathrm{N} \\
47,645^{\circ} \mathrm{W}\end{array}$ & $\begin{array}{l}40,597^{\circ} \mathrm{N} \\
47,752^{\circ} \mathrm{W}\end{array}$ & $\begin{array}{l}40,597^{\circ} \mathrm{N} \\
47,645^{\circ} \mathrm{W}\end{array}$ & $\begin{array}{l}40,490^{\circ} \mathrm{N} \\
47,752^{\circ} \mathrm{W}\end{array}$ & $\begin{array}{l}40,490^{\circ} \mathrm{N} \\
47,645^{\circ} \mathrm{W}\end{array}$ \\
\hline FIC Surface Level & $\begin{array}{l}1,050 \mathrm{~cm} \\
(414 \mathrm{in})\end{array}$ & $\begin{array}{l}904 \mathrm{~cm} \\
\text { (356 in) }\end{array}$ & N/A & $\begin{array}{l}1,040 \mathrm{~cm} \\
(408 \mathrm{in})\end{array}$ & $\begin{array}{l}965 \mathrm{~cm} \mathrm{(380} \\
\text { in) }\end{array}$ & $\begin{array}{l}1,020 \mathrm{~cm} \\
(403 \mathrm{in})\end{array}$ \\
\hline $\begin{array}{l}\text { Manual Tape Surface } \\
\text { Level }\end{array}$ & N/A & N/A & $\begin{array}{l}597 \mathrm{~cm} \mathrm{(235} \\
\text { in) }\end{array}$ & N/A & $N / A$ & N/A \\
\hline ENRAF Gauge & No & No & No & No & No & No \\
\hline Waste Type & DSSF & DN & DN/PD & DN & DN/PD & DN \\
\hline Total Waste & $\begin{array}{l}4,320 \mathrm{~kL} \cdot \\
(1,140 \mathrm{kgal})\end{array}$ & $\begin{array}{l}3,710 \mathrm{~kL} \\
(979 \mathrm{kgal})\end{array}$ & $\begin{array}{l}2,450 \mathrm{~kL} \\
(647 \mathrm{kgal})\end{array}$ & $\begin{array}{l}4,240 \mathrm{~kL} \\
(1,120 \mathrm{kgal})\end{array}$ & $\begin{array}{l}3,940 \mathrm{~kL} \\
(1,040 \mathrm{kgal})\end{array}$ & $\begin{array}{l}4,200 \mathrm{~kL} \\
(1,110 \mathrm{kgal})\end{array}$ \\
\hline Supernate & $\begin{array}{l}3,970 \mathrm{~kL} \\
(1,050 \mathrm{kgal})\end{array}$ & $\begin{array}{l}3,700 \mathrm{~kL} \\
\text { (978 kgal) }\end{array}$ & $\begin{array}{l}1,080 \mathrm{~kL} \\
(284 \mathrm{kgal})\end{array}$ & $\begin{array}{l}3,150 \mathrm{~kL} \\
(833 \mathrm{kgal})\end{array}$ & $\begin{array}{l}2,830 \mathrm{~kL} \\
(747 \mathrm{kgal})\end{array}$ & $\begin{array}{l}3,080 \mathrm{~kL} \\
(813 \mathrm{kgal})\end{array}$ \\
\hline $\begin{array}{l}\text { Drainable Interstitial } \\
\text { Llquid }\end{array}$ & $\begin{array}{l}7.57 \mathrm{~kL}(2 \\
\text { kgal) }\end{array}$ & -0 & $\begin{array}{l}140 \mathrm{~kL} \quad(37 \\
\mathrm{kgal})\end{array}$ & $\begin{array}{l}185 \mathrm{~kL} \text { (49 } \\
\text { kgal) }\end{array}$ & $\begin{array}{l}110 \mathrm{~kL} \text { (29 } \\
\text { kgal) }\end{array}$ & $\begin{array}{l}159 \mathrm{~kL} \text { (42 } \\
\text { kgal) }\end{array}$ \\
\hline $\begin{array}{c}\text { Sludge } \\
-\end{array}$ & $\begin{array}{l}318 \mathrm{~kL} \\
(84 \mathrm{kgal})\end{array}$ & $\begin{array}{l}3.79 \mathrm{~kL} \\
(1 \mathrm{kgal})\end{array}$ & $\begin{array}{l}1,370 \mathrm{~kL} \\
\text { (363 kgal) }\end{array}$ & $\begin{array}{l}678 \mathrm{~kL} \\
(179 \mathrm{kgal})\end{array}$ & $\begin{array}{l}1,120 \mathrm{~kL} \\
(297 \mathrm{kgal})\end{array}$ & $\begin{array}{l}799 \mathrm{~kL} \\
\text { (211 kgal) }\end{array}$ \\
\hline Integrity Category & Sound & Sound & Sound & Sound & Sound & Sound \\
\hline Watch List Status & Hydrogen & Not Listed & Not Listed & Not Listed & Not Listed & Not Listed \\
\hline Temperature & $\begin{array}{l}40.0^{\circ} \mathrm{C} \\
\left(104^{\circ} \mathrm{F}\right)\end{array}$ & $\begin{array}{l}19.4^{\circ} \mathrm{C} \\
\left(67^{\circ} \mathrm{F}\right)\end{array}$ & $\begin{array}{l}18.9^{\circ} \mathrm{C} \\
\left(66^{\circ} \mathrm{F}\right)\end{array}$ & $\begin{array}{l}22.2^{\circ} \mathrm{C} \\
\left(72^{\circ} \mathrm{F}\right)\end{array}$ & $22.2^{\circ} \mathrm{C}\left(72^{\circ} \mathrm{F}\right)$ & $35.6^{\circ} \mathrm{C}\left(96^{\circ} \mathrm{F}\right)$ \\
\hline Tank Use & CWHT & EVFD & DRCVR & DRCVR & DRCVR & SRCVR \\
\hline
\end{tabular}




\subsubsection{1-SY Tank Farm}

The 241-SY Tank Farm consists of three tanks and is the only double-shell tank farm in the 200 West Area. These tanks have received various wastes from various operations and facilities in the 200 West Area (Welty, 1988). Tank 241-SY-101 was used as a double-shell slurry holding tank for the 242-S Evaporator/Crystallizer. Tank SY-102 received waste generated by 200 West Area single-shell jet pumping operations. This tank was the feed tank for the 242-S Evaporator/Crystallizer and serves as a cross-site tie for transfers between the 200 Areas. Tank SY-103 was used for the storage of high-strontium concentrated liquor. Table 4-29 provides status of the individual tanks as of March 1994 (Hanlon).

As noted in Table 4-29, Tanks 241-SY-101 and -SY-103 are on the flammable gas watch list. A principal safety issue for Tank SY-101 has been the episodic release of substantial quantities of gases that approach lower flammability limits in the tank vapor space (Edwards, 1993). Major cyclic variations of the waste surface level, the most dynamic surface level changes observed in Hanford Site tanks varying from 10.2 to $27.9 \mathrm{~cm}$ (4 to 11 in.), have been measured in this tank. Attempts in the 1980's to mitigate this problem included lancing the waste in Tank SY-101 with water and air. However, these efforts were stopped after surface level increases were observed (Brager, 1993). A more recent technique providing encouraging results was the insertion and operation of a pump designed to recirculate tank waste. Full-scale testing of the pump was completed in April 1994 and the pump has entered the operational phase (Hanlon, 1994). Surface level data from Tank SY-103 also show cyclic variations; however, the variations are much smaller than those that had been observed with Tank SY-101 (Brager, 1993).

Table 4-29. 241-SY Tank Farm Status.

\begin{tabular}{|c|c|c|c|}
\hline Tank : $\because$ & $241-S Y=101$ U & $241-\$ Y-102$ & $2415 Y-103$ \\
\hline $\begin{array}{l}\text { Hanford } \\
\text { Coordinates }\end{array}$ & $\begin{array}{l}36,405^{\circ} \mathrm{N} \\
75,455^{\circ} \mathrm{W}\end{array}$ & $\begin{array}{l}36,405^{\circ} \mathrm{N} \\
75,562^{\circ} \mathrm{W}\end{array}$ & $\begin{array}{l}36,298^{\circ} \mathrm{N} \\
75,562^{\circ} \mathrm{W}\end{array}$ \\
\hline FIC Surface Level & $1,020 \mathrm{~cm}(402 \mathrm{in})$ & $671 \mathrm{~cm}(264 \mathrm{in})$ & $686 \mathrm{~cm}(270 \mathrm{in})$ \\
\hline $\begin{array}{l}\text { Manual Tape } \\
\text { Surface Level }\end{array}$ & N/A & N/A & N/A \\
\hline ENRAF Gauge & No & No & No \\
\hline Waste Type & $\mathrm{CC}$ & DN/PT & $\mathrm{CC}$ \\
\hline Total Waste & $4,200 \mathrm{~kL}(1,110 \mathrm{kgal})$ & $2,740 \mathrm{~kL}(725 \mathrm{kgal})$ & $2,800 \mathrm{~kL}(741 \mathrm{kgal})$ \\
\hline Supemate & $56.8 \mathrm{~kL}$ (15 kgal) & $2,480 \mathrm{~kL}(654 \mathrm{kgal})$ & $621 \mathrm{~kL}$ (164 kgal) \\
\hline $\begin{array}{l}\text { Drainable Interstitial } \\
\text { Liquid }\end{array}$ & $897 \mathrm{~kL}$ (237 kgal) & -0 & -0 \\
\hline Sludge & $\begin{array}{l}2,010 \mathrm{~kL} \quad(530 \mathrm{kgal}) \\
\text { DSS }\end{array}$ & -0 & $\begin{array}{l}2,170 \mathrm{~kL} \quad(573 \mathrm{kgal}) \\
\text { DSS }\end{array}$ \\
\hline Integrity Category & Sound & Sound & Sound \\
\hline Watch List Status & Hydrogen & Not Listed & Hydrogen \\
\hline Temperature & $51.1^{\circ} \mathrm{C}\left(124^{\circ} \mathrm{F}\right)$ & $18.9^{\circ} \mathrm{C}\left(66^{\circ} \mathrm{F}\right)$ & $37.2^{\circ} \mathrm{C}\left(99^{\circ} \mathrm{F}\right)$ \\
\hline Tank Use & CWHT & DRCVR & CWHT \\
\hline
\end{tabular}




\subsubsection{1-AY and $-A Z$ Tank Farms}

The 241-AY and -AZ Tank Farms consist of two tanks each located in the 200 East Area. Tanks in these farms were designed to provide aging-waste storage space for high-level radioactive waste generated at the PUREX Plant (Welty, 1988). Some of these tanks have also been used as slurry receivers. AZ-101 was first used in 1977 as a receiver tank for highstrontium-content waste. The $A Z$ tanks currently store high-level waste generated at PUREX. The AY tanks are currently storing low-level mixed waste. One of the AY tanks is being maintained as an emergency backup for the $A Z$ tanks. These tanks feature a heat-treated, stress-relieved primary steel liner and a nonstress-relieved outer steel liner (WHC, 1993). Stress gages have been installed on the AY tanks to measure any stress change occurring while the tanks are in service. To accommodate high-heat generating wastes, airlift circulators, steam coils, and exhaust condensers have been installed to minimize the probability of a loss of integrity. The A.Y and $A Z$ tanks must not be filled past a maximum of $914 \mathrm{~cm}$ (360 in.) because of process pipelines that penetrate the side of the tanks. The $A Y, A Z, A$, and $A X$ Tank Farms share common auxiliary services and were initially connected to the same tank ventilation system. The $A$ and AX Tank Farm tie-ins have since been isolated. Table 4-30 provides status of the individual tanks as of March 1994 (Hanlon).

Table 4-30. 241-AY and AZ Tank Farm Status.

\begin{tabular}{|c|c|c|c|c|}
\hline $\operatorname{Tank}:=\quad \because \quad /$ & $241-A Y 101$ & 241 AY 102 & 241 Az-101 & $241, \mathrm{Az} 102$ \\
\hline $\begin{array}{l}\text { Hanford } \\
\text { Coordinates }\end{array}$ & $\begin{array}{l}41,733.11^{\circ} \mathrm{N} \\
47,836.54^{\circ} \mathrm{W}\end{array}$ & $\begin{array}{l}41,626.06^{\circ} \mathrm{N} \\
47,836.54^{\circ} \mathrm{W}\end{array}$ & $\begin{array}{l}42,080^{\circ} \mathrm{N} \\
47,506^{\circ} \mathrm{W}\end{array}$ & $\begin{array}{l}42,080^{\circ} \mathrm{N} \\
47,613^{\circ} \mathrm{W}\end{array}$ \\
\hline FIC Surface Level & N/A & $\begin{array}{l}869 \mathrm{~cm} \mathrm{(342} \\
\text { in) }\end{array}$ & N/A & N/A \\
\hline $\begin{array}{l}\text { Manual Tape Surface } \\
\text { Level }\end{array}$ & $\begin{array}{l}846 \mathrm{~cm}(333 \\
\text { in) }\end{array}$ & N/A & $\begin{array}{l}876 \mathrm{~cm} \mathrm{(345} \\
\text { in) }\end{array}$ & $\begin{array}{l}894 \mathrm{~cm} \mathrm{(352} \\
\text { in) }\end{array}$ \\
\hline ENRAF Gauge & No & No & No & No \\
\hline Waste Type & $D C$ & DN & Aging & Aging \\
\hline Total Waste & $\begin{array}{l}3,360 \mathrm{~kL}(888 \\
\text { kgal) }\end{array}$ & $\begin{array}{l}3,560 \mathrm{~kL}(941 \\
\text { kgal) }\end{array}$ & $\begin{array}{l}3,590 \mathrm{~kL}(949 \\
\mathrm{kgal})\end{array}$ & $\begin{array}{l}3,660 \text { kL (967 } \\
\text { kgal) }\end{array}$ \\
\hline Supernate & $\begin{array}{l}3,050 \mathrm{~kL}(805 \\
\text { kgal) }\end{array}$ & $\begin{array}{l}3,440 \mathrm{~kL}(909 \\
\text { kgal) }\end{array}$ & $\begin{array}{l}3,460 \mathrm{~kL}(914 \\
\mathrm{kgal})\end{array}$ & $\begin{array}{l}3,300 \mathrm{~kL}(872 \\
\text { kgal) }\end{array}$ \\
\hline $\begin{array}{l}\text { Drainable Interstitial } \\
\text { Liquid }\end{array}$ & $\begin{array}{l}7.57 \text { kL (2 } \\
\text { kg̣al) }\end{array}$ & -0 & -0 & $\begin{array}{l}15.1 \mathrm{~kL} \mathrm{(4} \\
\mathrm{kgal})\end{array}$ \\
\hline Sludge & $\begin{array}{l}314 \mathrm{~kL}(83 \\
\mathrm{kgal})\end{array}$ & $\begin{array}{l}121 \mathrm{~kL}(32 \\
\mathrm{kgal})\end{array}$ & $\begin{array}{l}132 \mathrm{~kL}(35 \\
\mathrm{kgal})\end{array}$ & $\begin{array}{l}360 \mathrm{~kL}(95 \\
\mathrm{kgal})\end{array}$ \\
\hline Integrity Category & Sound & Sound & Sound & Sound \\
\hline Watch List Status" & Not Listed & Not Listed & Not Listed & Not Listed \\
\hline Temperature & $\begin{array}{l}48.9 \text { to } 76.7^{\circ} \mathrm{C} \\
\left(120 \text { to } 170^{\circ} \mathrm{F}\right)\end{array}$ & $\begin{array}{l}48.9 \text { to } 76.7^{\circ} \mathrm{C} \\
\left(120 \text { to } 170^{\circ} \mathrm{F}\right)\end{array}$ & $\begin{array}{l}48.9 \text { to } \\
76.7^{\circ} \mathrm{C} \\
(120 \text { to } \\
\left.170^{\circ} \mathrm{F}\right)\end{array}$ & $\begin{array}{l}48.9 \text { to } 76.7^{\circ} \mathrm{C} \\
\left(120 \text { to } 170^{\circ} \mathrm{F}\right)\end{array}$ \\
\hline Tank Use & DRCVR & DRCVR & CWHT & DRCVR \\
\hline
\end{tabular}




\subsection{SAMPLING PROTOCOLS}

Sampling is carried out based on the type of waste in each individual tank. The types of sampling efforts that support various TWRS activities include push-mode and rotary-mode core sampling, grab sampling, auger sampling, and various types of vapor sampling. A general description of each type of sample and sampling procedure follows (Bell, 1994).

\subsection{CORE SAMPLES}

Push mode and rotary mode are two core sampling methods used to obtain solid and/or supernatant liquid samples of the high-level waste materials remaining in the underground waste tanks. Sludge, salt cake, and liquid are the three general waste phases in the various tanks. The samples are obtained from the tanks using specially designed core-sampling trucks and sampling devices. A cylindrical cross-section of the tank contents below the riser used for sampling is obtained by pushing or rotating a $2.5 \mathrm{~cm} \times 48 \mathrm{~cm}$ (1 in. $\times 19$ in.) stainless steel sampler bit (Figure 5-1) into the waste. The usable volume of the sampler is about $187 \mathrm{~mL}$. Push-mode sampling is performed on tanks containing liquids or soft sludge. Rotary-mode sampling is primarily used to sample sludge or hard salt cake, although it may be used to sample the other waste types. During a sampling event, the stainless steel sampler is lowered into the waste by a hydraulically powered drill string. A spring-loaded piston is used to create negative pressure which helps draw the sample into the waste column. Drill ram hydrostatic pressure is continuously monitored during the sampling operation to measure waste resistance. Core samples are repeatedly taken until the bottom of the tank is reached. The waste approximately $7.6 \mathrm{~cm}$ ( $3 \mathrm{in}$.) from the tank bottom cannot be sampled due to design and safety constraints of the sampling method.

\subsection{AUGER SAMPLES}

Auger samples are used to screen the surface of organic, ferrocyanide, and gasgenerating watch list tanks, and may also substitute for core samples in tanks containing less than $20 \mathrm{~cm}$ to $30 \mathrm{~cm}$ of waste. Auger samples are taken using a stainless steel, hand-turned auger-bit contained in a sleeve in a method similar to wood boring or ice augering. This sampling method has some significant limitations: very fluid wastes cannot be augered since the sample cannot be properly captured in the sampler. The ability to check vertical variability is lost because the augering procedure disturbs the waste in the sample and affects recovery. Also, auger samples are limited to approximately the first $20 \mathrm{~cm}$ of solids on the tank waste surface and are not planned for all tanks.

\subsection{SUPERNATE AND SOFT SLURRY GRAB SAMPLES}

A special sampling bottle that is contained in a cage can be used to obtain liquid or soft slurry samples of the waste. The bottle is stoppered and lowered to the desired level. The bottle is then opened so that it fills with liquid (approximately $100 \mathrm{~mL}$ ) and is then restoppered and retrieved from the tank. This "bottle-on-a-string" sampling procedure conforms to ASTM (1973). The sample bottles may not be filled completely due to safety procedures designed to limit personnel exposure to hazardous ionizing radiation. 
Figure 5-1. Stainless Steel Core Sampler.

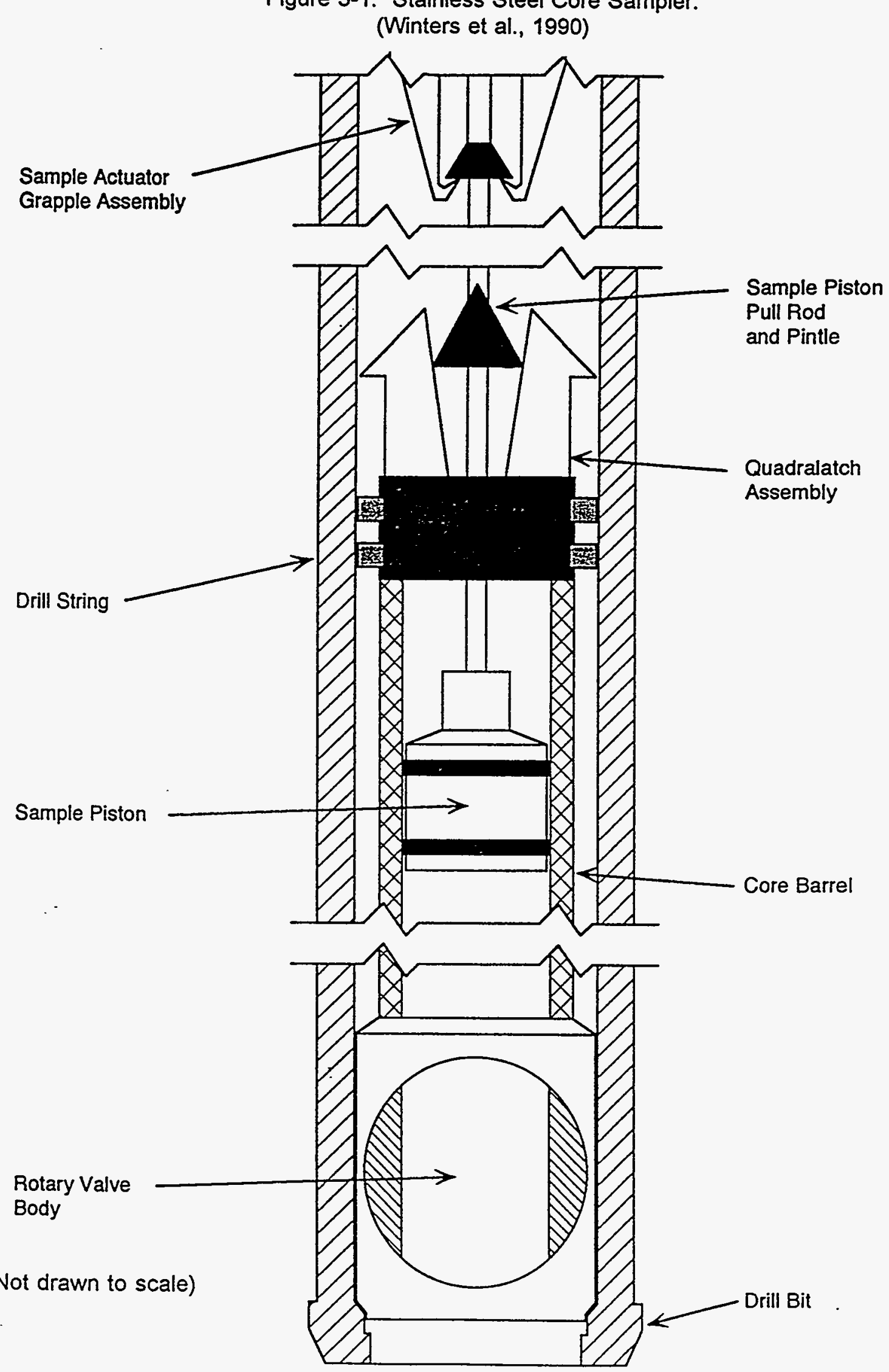




\subsection{VAPOR SAMPLING}

Flammable and/or noxious vapors and gases may be generated and released from the high-level radioactive waste within the 200 Area tank farms. There are several different measuring devices, procedures, and instruments for vapor sampling. The tank vapor sampling currently being conducted may be classified by purpose into three general types: ferrocyanide tank flammability and health hazards vapor sampling, heated-tube vapor sampling, and exhauster air permit vapor sampling. These apply to watch-list tanks in general.

Ferrocyanide tank flammability and health hazards vapor sampling are required before any work inside the ferrocyanide watch-list tanks can be performed. A safety assessment specifically outlines the requirements for this sampling, which involves a check of flammability of the headspace using a combustible gas meter and a check of the organic vapor concentration using an organic vapor monitor within the tank. Sorbent tubes are used to measure certain hazardous compounds, including hydrazine, ammonia, hydrogen cyanide, and nitrogen oxides in the tank dome space.

The Tank Vapor Issue Resolution Program developed the vapor sampling system using heated-tube vapor sampling to resolve health and safety issues. The heated-tube vapor sampling uses a heated sampling probe, heated sample transfer lines, and a heated manifold to eliminate the condensation of vapors. At this time, all ferrocyanide and organic watch-list tanks are scheduled to be sampled by this method.

Exhauster air permit vapor sampling is used to support exhauster use during rotary-mode core drilling. The vapors in a waste tank must be sampled and analyzed before using the exhauster to acquire a State air permit to operate an exhauster in a tank.

There are three processes for obtaining vapor samples. A sample may be passed through a device that traps or concentrates certain vapors and gases, a sample may be collected in an appropriate container, or the vapor may be analyzed in situ. Several methods exist for vapor sampling. These and other methods are currently being developed. Further discussion of existing methods is presented in the Tank Waste Remediation System Tank Waste Analysis Plan (Bell, 1994).

\subsection{CHÁIN-OF-CUSTODY RECORD}

Chain-of-custody records are completed for each sample obtained (Simpson et al., 1993). This ensures that each sample is uniquely identified, safely and properly transported from the field to the laboratory, and traceable during transport. Also, the records provide a brief description of the sample and its origin. Radiation survey data are collected for each sample to ensure proper sample containment. Radiation values are measured on the top, sides, and bottom of the shipping cask containing the sample and reported on the chain-of-custody record. Values greater than $100 \mathrm{mrem} / \mathrm{hr}$ are considered unsafe. 


\subsection{ANALYTICAL METHODS SUMMARY}

\subsection{PREPARATIVE METHODS}

Most instrumental techniques require some form of sample preparation before analysis. For metals, this normally involves digestion with strong mineral acids or fusion with potassium hydroxide. Extraction with methylene chloride or hexane is used for semivolatile organics, and purge and trap or direct injection may be used for volatile organics. Gross activities (alpha, beta, or gamma), and others such as liquid scintillation, may be performed directly on the sample or digestate; however, measurements of specific radionuclides usually require a series of separation steps after digestion.

\subsubsection{Direct/Dilution}

Some of the analytes in waste or environmental samples undergo physical or chemical changes when exposed to the acid digestion or potassium hydroxide $(\mathrm{KOH})$ fusion dissolution processes. Some of the more volatile constituents such as mercury, arsenic, organic carbon, and organic halides will be lost if subjected to rigorous digestion techniques. In such cases, the sample is analyzed directly. In the case of analyses that require liquid sample introduction, a water leach of the solid sample is used.

\subsection{2 $\mathrm{pH} / \mathrm{OH}^{-}$}

Hydroxide ion concentration is determined by titrating an aliquot sample with nitric acid to a potentiometric end point, using a combination $\mathrm{pH}$ electrode as an indicator. For $\mathrm{OH}^{-}$analysis, hydrolyzable anions, (e.g., carbonate, aluminate, and phosphate) if present, will bias the results high. Chromate ion will also cause high results. Some of these anions may be removed from solution by precipitation with barium chloride. Even then, however, these anions will cause a high bias above certain limits. Ions of other weak acids (e.g., cyanide and borate) may also interfere and must be absent.

For $\mathrm{H}^{+}$analysis, the hydrolyzable cations $(\mathrm{U}, \mathrm{Pu}, \mathrm{Fe}, \mathrm{Al}$, and $\mathrm{Zr}$ ) are complexed with a neutral solution of KF and ammonium oxalate. Free acid can then be titrated with a standard base with minimal interference from the hydrolysis reactions. A pH electrode and meter are used to measure the $\mathrm{pH}$ at the end point. The quantity of hydrolyzable metal cations should not exceed $2.2 \mu \mathrm{mol}$ of a sample that is $2.2 \mathrm{M}$ or less in these metals. If chromium (III) is present, it will react very slowly with the oxalate and may cause poor results.

\subsubsection{Acid Digestion}

Acid digestions are generally used to prepare samples for metals or radiochemical analyses. An accurately weighed portion of sample (usually 1 to 2 grams) is digested in either nitric or hydrochloric acid. The sample is heated until the volume is reduced to a low level, at which point more acid is added. The process is repeated several times to ensure adequate dissolution. After digestion, the sample is filtered and diluted to a known volume with deionized and/or distilled water. It is important to note that acid digestions may not dissolve all metals, 
particularly silicates. For this reason, samples may be prepared using both an acid digestion and a potassium hydroxide fusion technique. Comparison of the results can then reveal information concerning analyte solubility.

\subsubsection{Potassium Hydroxide Fusion}

A sample preparation technique that is sometimes used for the determination of metals in solids is the fusion technique. Samples may be fused in a variety of fluxes including borates, fluorides, potassium hydroxide, and carbonates. The fusion technique most commonly used for the preparation of tank wastes is potassium hydroxide fusion.

Fusion is accomplished by mixing a suitable amount of potassium hydroxide with an accurately weighed portion of the sample (usually in about a 1:20 ratio). The mixture is then heated in a nickel crucible at about 500 to $600{ }^{\circ} \mathrm{C}$. Once the mixture becomes molten, it is maintained at this temperature until the sample matrix is dissolved. The melted sample is then poured into a dilute acid solution, either hydrochloric, nitric, or aqua regia, or allowed to cool and solidify in the crucible. The liquid is then filtered and diluted to an appropriate volume, depending on the analysis for which it is intended.

Fusion methods have some limitations. In many cases, high blank levels, caused either by low reagent purity or contamination from the crucibles, may adversely affect detection limits. High dilution factors also adversely affect the quantitation of insoluble analytes present at low levels. The sample digestate will contain an elevated level of the crucible material. Multiple fusions may be required to adequately prepare a sample for all analytes of concern to the various data users.

Another limitation of the fusion method is the loss of volatile components. If the flux or temperature used for the fusion is not appropriate, or if the fusion time is excessive, volatile compounds of analytes such as arsenic, mercury, selenium, boron, and iodine will be lost. The advantage of fusion is the ability to completely dissolve compounds or elements that are insoluble in acids, although noble metals may still be insoluble.

\subsubsection{Volatile and Semivolatile Organic Compound Extraction}

Volatile organics are determined by the purge and trap method. This involves bubbling an inert gas through a water sample contained in a purging vessel. The vapor is swept through a sorbent trap to collect the organics. After purging is completed, the trap is heated and backflushed with the same inert gas to desorb the compounds onto a chromatographic column. Solid sample, or high level samples, are first diluted or extracted in methanol. The method detection limits depend on the sample matrix but generally range from 1 to $10 \mu \mathrm{g} / \mathrm{L}$.

Samples requiring semivolatile organics analysis are first extracted with methylene chloride at a pH above 11 and again at a pH below 2 . The extract is then concentrated and analyzed by gas chromatography/mass spectrometry. The detection limits vary between about 5 and $50 \mu \mathrm{g} / \mathrm{L}$ for water matrixes and 50 to $1,600 \mu \mathrm{g} / \mathrm{L}$ for soils. 


\subsection{ATOMIC SPECTROSCOPIC TECHNIQUES}

Atomic spectroscopy techniques in general detect the electromagnetic energy that is omitted or absorbed by an electron that is moving between ground and excited states. Thermal energy is used to create a plasma in which the atoms are free of molecular or crystalline forces; energy emitted or absorbed by atoms will most likely be characteristic of the atom itself, and not the result of making or breaking molecular or crystalline bonds. The atomic absorption techniques differ mainly in the ways in which the atom is excited and the ways in which the energy absorption or emission is detected and measured. Commonly used atomic spectroscopy are: inductively coupled plasma (ICP)(the sole method used for analyzing tank waste that measures emission; all others measure absorption), flame atomic absorption (FAA), graphite furnace atomic absorption (GFAA), and gaseous hydride atomic absorption (GHAA). The different techniques have advantages and disadvantages for particular analytes and matrixes.

\subsubsection{Inductively Coupled Plasma Spectroscopy}

The majority of the metals found in hazardous wastes and environmental samples are determined by ICP. This technique uses an excitation source that transforms a sample from a solution into a plasma of atoms and ions. After returning to the ground state, these excited particles produce electromagnetic radiation which is then dispersed by a diffraction grating into various wavelengths, depending on the element. A response proportional to analyte concentration at a given atomic emission line for each element is then resolved electronically.

Samples are introduced into the system through a nebulizer, converting the liquid sample into extremely fine droplets, which are then forced into the plasma torch. The torch assembly consists of a quartz inner tube surrounded by an outer tube. The entire assembly is surrounded by a radio frequency (RF) coil which converts the vapor into a plasma. Normally, the temperature of this plasma reaches 6,000 to $8,000^{\circ} \mathrm{C}$.

Atomic emissions (light) from the plasma, pass through a fine slit and are separated into various wavelengths by a grating. The angle of this grating determines the wavelength of radiation falling onto a photomultiplier tube, which detects the light. If the instrument is sequential, the grating is moved through the appropriate wavelengths of all the elements analyzed and the radiation is detected by a single photomultiplier tube. Simultaneous instruments rely on an array of photomultipliers assembled at fixed locations, depending on the wavelength required.

The detection limits obtainable by ICP vary from a few parts per billion to several hundred parts per million, depending on the element and the matrix of the element determined.

\subsubsection{Flame AA}

In flame AA, a fine aerosol of the sample solution, following nebulization, is introduced into a flame where it is desoluated, vaporized, and atomized. Some of the atoms of a sample will absorb energy at a particular wavelength and become excited to a higher electronic state; or, they may absorb energy from the flame and become thermally excited. The latter process is the principle for flame emission atomic absorption. The former, atomic absorption (AA), is more commonly used in the analysis of hazardous waste. In flame $A A$, the ground state is achieved by nebulization, followed by introduction into either a nitrous oxide/acetylene flame, or an air/acetylene flame. Following Beer's Law, the concentration of an analyte is proportional to the absorbance. This technique is required for quantitating cesium concentration in a sample.

LATA-TCR-9491, Rev. 0 


\subsubsection{Graphite Fumace AA}

The relatively poor detection limits for flame $A A$ are due to the inefficiency in converting all of the elements in a sample to atomic vapor. To compensate for this inefficiency, the graphite furnace AA (GFAA) technique was developed.

In the furnace technique, a few microliters of sample are placed in a graphite tube and thermally atomized by resistive heating to produce a cloud of atomic vapor. The light path passes through the tube and a peak of absorbance versus time is measured. Because furnace atomizers have conversion efficiencies of 100 to 1,000 times that of flame atomizers, the detection limits are much lower. Typically, the detection limits for GFAA are in the lower parts per billion range.

The primary disadvantage of GFAA is that it can be subject to severe matrix interference effects. These effects can be partially compensated for through the use of internal standards or the method of standard addition to directly relate readings to calibration. In fact, the EPA Contract Laboratory Program (CLP) procedures for GFAA mandate the use of such methods for minimization of matrix effects.

\subsubsection{Gaseous Hydride Atomic Absorption Spectroscopy}

Gaseous hydride AA (GHAA) is an alternative to the furnace method. In this method, the analyte is converted to the hydride using sodium borohydride. This gaseous hydride is then swept into a cell and the absorbance of the particular element measured as a function of concentration. The hydride method can determine up to four elements simultaneously.

\subsubsection{Advantages of AA}

The advantage of all atomic absorption methods over ICP lies in the specificity of the method. Because the technique measures only a very narrow bandwidth of radiation, the problem of spectral overlap is minimal. The primary advantage is in the ability of the technique to capture and quantitate volatile metal species at low concentrations. ICP detection levels are high in comparison due to loss from volatilization.

\subsection{CHROMATOGRAPHIC TECHNIQUES}

Chromatography is the general name given to the methods by which two or more compounds in a sample are physically separated by distribution between two phases: a stationary phase and a mobile phase. A number of chromatographic techniques have been developed for the separation and analysis of organic and inorganic compounds. The primary methods used for the separation of organic constituents in hazardous waste and environmental samples are gas chromatography (GC). Gas chromatography/mass spectrometry (GC/MS) is frequently used for quantitation of the species present. Ion chromatography (IC) is generally used for the determination of cations and anions. High performance liquid chromatography (HPLC) is often used for the separation of nonvolatile or thermally unstable organic compounds. 


\subsubsection{Gas Chromatography}

Gas chromatography (GC) is primarily used for the analysis of pesticides, herbicides, PCBs, and volatile organics. GC systems include a heated injection port, a chromatographic column, and a detector. A sampling device first introduces a prepared sample through the injection port. The vaporized sample then moves into the GC column, which separates the various compounds according to physical properties such as polarity, solubility, and volatility. The carrier gas is usually nitrogen or helium. Each column employs a gaseous mobile phase and a liquid stationary phase to separate volatile or semivolatile components from a mixture. The stationary phase is a liquid that has been coated on an inert granular solid, or in capillary columns, on the column itself. The stationary phase varies, depending on the type of compound analyzed. Quantitation is accomplished by spectral interpretation of time-dependent elution fractions against calibrations using the same operating parameters.

Several types of detectors are used, depending on the application. Table 6-1 lists the types of detectors commonly used for the analysis of hazardous wastes.

Table 6-1. GC Detector Applications.

\begin{tabular}{|c|c|c|}
\hline Detector & Compounds Detected & $\begin{array}{l}\text { Defection } \\
\text { Limits }\end{array}$ \\
\hline Flame Ionization Detector (FID) & Most carbon-containing compounds & $0.01 \mu \mathrm{g} / \mathrm{L}$ \\
\hline Photoionization Detector (PID) & Varies, depending on ionization source & $0.001 \mu \mathrm{g} / \mathrm{L}$ \\
\hline Electrolytic Conductivity & Organic Halogens & TBD \\
\hline Electron Capture Detector (EC) & Pesticides, organometallics & $0.01 \mu \mathrm{g} / \mathrm{L}$ \\
\hline
\end{tabular}

\subsubsection{Mass Spectrometry}

A gas chromatograph/mass spectrometer is essentially a gas chromatograph that utilizes a mass spectrometer for additional resolution. This is useful for samples that contain many organic compounds. Each GC elution fraction can be separated into the various constituents based on the mass of the constituents in that fraction. Since many organic compounds have the same mass, evaluation of spectra must include GC column interactions also.

\subsubsection{Ion Chromatography}

Ion chromatography (IC) permits the measurement of the amounts of various ions in an analytical sample, thereby achieving a quantitative chemical analysis. The method is applicable to the analysis of either, cations or anions. In typical equipment used in IC, the eluent is pumped through the entire system. Next, the sample is washed from the loop and onto the ion-exchange column as a discrete "plug." On the ion-exchange column, the sample ions compete for the exchange sites with the eluent ions. As each sample ion comes off the column and passes through the detector cell, the electronic signal from the detector changes and is recorded. 


\subsubsection{High Performance Liquid Chromatography}

High performance liquid chromatography (HPLC) is primarily used for the analyses of nonvolatile organics, such as citrate, EDTA, and HEDTA. HPLC involves the use of a very small particle size in column packing, with high pressures to increase the rate of flow and shorten the separation time to a few minutes. The solvent is moved through the system by constant flow or constant pressure pumps. An injector valve is used to introduce a small volume of sample onto the top of the column without interrupting the mobile-phase flow. After the column, a flow-through detector is employed to generate the chromatograph which is recorded.

\subsection{RADIOCHEMICAL METHODS}

Three types of detectors are.commonly used for detection of radiation: gas proportional detectors, scintillation detectors, and solid state detectors. The application of these detectors vary between laboratories but in general, gas proportional detectors are used for measurement of gross alpha and beta radiation, scintillation detectors for beta and gamma-ray detection, and solid state detectors for $\mathrm{X}$-ray and gamma-ray detection.

\subsubsection{Gas Proportional Detectors}

Gas proportional counters consist of a tube filled with a suitable gas (usually an argonmethane mixture) with a very thin window made of aluminized mylar or beryllium. Radiation entering the detector causes the gas to ionize, which in turn causes a change in the voltage of a wire placed in the center of the tube. This change in voltage is proportional to the number of particles entering the detector. The signal produced by the voltage change is electronically amplified and reported as a function of counting time.

Gas proportional measurements are commonly used to determine the level of gross alpha or gross beta activity in a sample. In addition, various individual radionuclides can be chemically separated and counted by gas proportional counting. Gross alpha and beta measurements are useful for screening to determine whether further analysis for specific radionuclides is needed. Accurate determination of gross alpha or beta requires careful preparation of standards to determine self-absorption. In addition, the radionuclides used for standards must have energies relatively similar to those being measured. For example, at Hanford, the most common beta emitters are ${ }^{90} \mathrm{Sr}$ and ${ }^{137} \mathrm{Cs}$. A common standard for gross beta determinations is ${ }^{60} \mathrm{Co}$, which has significantly different energy from either ${ }^{137} \mathrm{Cs}$ or ${ }^{90} \mathrm{Sr}$. If ${ }^{60} \mathrm{Co}$ is used for gross beta calibration without corrections for different efficiencies, a significant bias will result if the primary radionuclide in the sample is not ${ }^{60} \mathrm{Co}$.

\subsubsection{Gamma Energy Analysis}

Gamma spectrometers utilize one of two types of detectors: intrinsic germanium (Ge) or thallium-activated sodium iodide [Nal(TI)]. In the germanium system, gamma photons enter the detector and produce ionized atoms. The Nal systems utilize a photomultiplier tube to convert the gamma photons into pulses of light, which then ionize the detector atoms. As in the case of the gas proportional detector, the collection of the ions from these detectors results in a change in the bias voltage that is applied to the detector. This change in bias is then processed in a 
pulse-height analyzer. The amplified signals are then displayed as spectrum of energy versus intensity.

The advantage of gamma energy analysis (GEA) is that a minimum of sample preparation is required. In addition, most gamma systems can measure all gamma-emitting isotopes simultaneously. A few of the isotopes measured by GEA at Hanford are ${ }^{134} \mathrm{Cs}^{137} \mathrm{Cs},{ }^{60} \mathrm{Co},{ }^{154} \mathrm{Eu}$, ${ }^{155} \mathrm{Eu},{ }^{106} \mathrm{Ru}$, and ${ }^{40} \mathrm{~K}$. Gamma radiation is released during decay in discrete photons or energy that fingerprints many radionuclides simultaneously. There are many overlapping spectral features in high activity samples requiring evaluation and/or specific separations before quantitation.

\subsubsection{Alpha Spectrometry}

Alpha spectrometers use a silicon semiconductor detector to measure alpha particles. The particles entering the detector cause the atoms to ionize. The ionization of the detector then allows the generation of an electrical pulse that is proportional to the energy of the alpha particles measured. The pulses are processed and displayed as a spectrum. Alpha particles have low penetrating power relative to other types of radiation of comparable energy due to a mass of 4 atomic mass units and a charge of +2 . Because of this, alpha spectrometry is conducted under a high vacuum and the samples are usually electrodeposited in a very thin layer. Several isotopes emit alpha particles with the same apparent energy (e.g., ${ }^{241} \mathrm{Am},{ }^{238} \mathrm{Pu}$, and ${ }^{240} \mathrm{Pu}$ ). Therefore, these isotopes are reported as pairs. Mass spectrometry is the only alternative.

Typically, the plutonium and uranium isotopes are measured by alpha spectrometry. At Hanford, the technique is used to determine the ratio of the various plutonium isotopes to each other. This ratio is then applied to the total alpha result obtained by proportional counting (after separation) to quantitate the plutonium activity of each isotope.

\subsubsection{Liquid Scintillation Counting}

Ion exchange or solvent extraction separations are used for nonvolatile nuclides (e.g., ${ }^{99} \mathrm{Tc}$ and ${ }^{79} \mathrm{Se}$ ). The beta emitters, ${ }^{14} \mathrm{C}$ and ${ }^{3} \mathrm{H}$, which decay by a very weak beta particle, are determined by liquid scintillation counting. In this technique, the radionuclide is usually distilled to remove it from nonvolatile radioactive materials and then dissolved or suspended in a scintillation cocktail. When radiation interacts with this organic scintillation solution, light is produced. This light is then detected and amplified by a photomultiplier tube. The signal from the photomultiplier tube (or tubes) is proportional to the level of activity for each radionuclide measured.

Liquid scintillation instruments are very stable and do not require constant recalibration. In most cases, the calibration is performed by the instrument manufacturer before shipping or onsite after major maintenance. The calibration is performed using the isotopes being measured if they are available. If an isotopic standard is unavailable, as in the case of ${ }^{79} \mathrm{Se}$, an isotope of similar energy is used for calibration. Several factors can affect the quality of data generated (i.e., matrixes which inhibit light transmission; fluorescence from the sample will enhance the signal). 


\subsection{THERMAL METHODS OF ANALYSIS}

Thermal methods of analysis include differential scanning calorimetry, thermogravimetric analysis, and differential thermal analysis.

Differential scanning calorimetry (DSC) measures the heat input necessary to keep a sample and a reference substance isothermal as the temperature is changed. DSC can provide precise values for heats of reaction.

Thermogravimetric analysis (TGA) involves continuously measuring the mass of a sample as a function of temperature. Plots of mass versus temperature are called thermogravimetric curves. The major use of TGA is in the precise determination of mass changes for several sequential reactions which may occur in the sample.

In differential thermal analysis (DTA), the difference in temperature between a sample and a reference as a function of temperature is measured while they are heated. These differences are observed when changes that involve a finite heat of reaction-such as chemical reactions, phase changes, or structural changes-occur in a sample. If the measured enthalpy is positive (endothermic reaction), the temperature of the sample will lag behind that of the reference. If the enthalpy is negative (exothermic reaction), the temperature of the sample exceeds that of the reference.

\subsection{PHYSICAL AND RHEOLOGICAL PROPERTIES}

Information concerning the physical and rheological properties of tank waste is obtained in order to achieve two objectives: to accurately assess the tank waste form for safety and inventory purposes, and to inform the process of developing the equipment used for waste retrieval and treatment.

Physical properties include density, particle size, and specific gravity of the various waste components such as supernate, slurry, and solids. The measurements of the organic and inorganic carbon content are also occasionally grouped with the physical analyses, although they are not properly "physical" tests:

Rheological properties include shear stress, shear strength, and viscosity. From these, flow properties such as critical flow rates and critical Reynold's numbers are calculated.

\subsubsection{Physical Properties}

Density. The samples are placed in preweighed, volume graduated centrifuge tubes which are then weighed and the volume determined. The density is the ratio of the mass to the volume.

Particle Size. Particle size analysis is performed by placing a small amount of sample in a dispersant, which is the liquid used to disperse and suspend the solid particles. The chemical nature of the dispersant is critical to the proper conduct of the test. A substance must be selected that will neither dissolve the sample particles, nor cause precipitation of new particles. The "mother liquor" from the tank is a good choice of dispersant since it is in chemical equilibrium with the solid material. After placing the sample in the dispersant, the prepared sample is placed 
in a particle analyzer which has a range of 0.5 to $150 \mu \mathrm{m}$. The apparatus measures particle size by passing a thin beam of laser light through the dispersant. The diameter of a particle is determined by the amount of light that is attenuated as the beam passes through the sample. The instrument will measure the short diameter of the particle, irrespective of its shape. This information can be used to generate number and volume probability densities. The number probability density is an indication of the average size of the particles based on the number of particles measured, whereas the volume probability density is an indication of the size of the particles which occupy most of the sample volume. A small difference between the number probability density and the volume probability density is an indication of relative uniformity of particle size; conversely, a large difference is an indication that most of the sample volume is occupjed by a few large particles and the remaining volume is occupied by many small particles (Jones, 1991).

Specific Gravity. The specific gravity of a substance is defined as the ratio of its density to that of water at $3.98^{\circ} \mathrm{C}$. A known volume of liquid sample is drawn into a preweighed vial. The sample density is calculated and then compared to that of water. The ratio of the two values is the specific gravity.

Determination of Carbon. Carbon compounds appear in several groups of compounds, among which are organic (those in which carbon is found in covalently bound chains), inorganic (carbonate, bicarbonate, and dissolved carbon dioxide), and volatile organic compounds. Volatile organic compounds are measured by chromatographic methods and are discussed in Section 6.3. Two methods are commonly used to determine organic and inorganic carbon: combustioninfrared, which measures organic and inorganic carbon (commonly called total carbon); and persulfate-ultraviolet oxidation, which measures organic carbon. Total inorganic carbon may be found by subtracting the organic carbon value from the total carbon value. In the combustioninfrared method, the carbon in the sample is oxidized with an oxidative catalyst, then measured by a nondispersive infrared analyzer. In the persulfate-ultraviolet oxidation method the organic carbon is oxidized to carbon dioxide in the presence of ultraviolet light. The sample may be analyzed directly by a nondispersive infrared analyzer, be reduced to methane and measured by a flame ionization detector, or be chemically titrated (EPA, 1992).

\subsection{2 - Rheological Properties}

Rheology is defined as the study of the deformation of matter. The rheological properties of tank waste are measured using a viscometer which is equipped with a measuring-drive head and a sensor system. The sample is placed in the gap between two concentric cylinders. A rotating torque is applied to the outer cylinder and the torque transmitted to the inner cylinder is measured using a calibrated spring. A slurry with a yield stress will clamp the inner cylinder to the outer until the applied torque exceeds the yield stress. This is called the yield point and denotes the point at which the sample begins to act as a fluid. The rate of rotation is measured along with the torque signal, and the two values are used to generate a shear rate versus shear stress curve. The data are fit to a nonlinear power law model to obtain the necessary parameters for the yield-pseudoplastic meological model. The equation for the model is:

$$
\begin{aligned}
\tau=\tau_{0} & +K(\text { shear rate })^{n} \\
\text { Where } \tau & =\text { shear stress } \\
\tau_{0} & =\text { yield stress (not a fit parameter) }
\end{aligned}
$$


The data are put into a computer model that describe the fluid's behavior in different size pipes and at different flow rates. The most desirable situation is that in which flow is turbulent, which will aid in holding the particles in suspension while the waste is being slurried from one tank to another, or from a tank to the processing facility.

\subsection{ANALYTICAL CONCERNS}

\subsubsection{Hold Time Considerations}

The use of holding times for regulatory purposes originated with the National Pollutant Discharge Elimination System (NPDES) analytical methods, which were concerned with the loss of trace level contaminants from waste water samples collected and analyzed under a self-monitoring program. The EPA Contract Laboratory Program (CLP)(EPA, 1989) implemented essentially the same holding times as NPDES, which were then adopted by SW-846 (EPA, 1992). Holding times and sample preservation techniques are currently under study by the EPA.

On August 31, 1993, the EPA published a final rule for testing and monitoring activities under Subtitle "C" for RCRA (EPA, 1990). In the rule, the EPA states that data generated after holding times are exceeded may be used to prove that a waste is hazardous, but not that the waste is nonhazardous. According to the EPA decision, samples must be extracted or analyzed within the specified hold times to be reflective of total concentrations. Results for samples analyzed outside of holding times must be considered to be minimum values only. Such data may be used to demonstrate that a waste is hazardous where it shows the concentration of a constituent to be above the regulatory threshold, but cannot be used to demonstrate that a waste is nonhazardous. In other words, holding times must be met whenever one is attempting to demonstrate that the concentration of a specific contaminant is less than a regulatory limit. However, for nonregulatory purposes, analyses obtained after a holding time has been exceeded do not necessarily invalidate the result.

The most effective time for demonstrating regulatory compliance will be after the waste has been mobilized, mixed, and retrieved. This greatly simplifies sampling and allows the accuracy needed for permitting and comparisons with regulatory limits.

A phased approach to waste analysis should ensure that all technical and regulatory Data Quality Objectives will ultimately be met without requiring a large number of purely regulation-based analyses before they are needed. The phased approach will be based upon criteria identified, defined, and agreed upon to address safety and process design needs. This approach is based upon the DOE's commitment to retrieve and treat all Hanford Site tank waste, and it is only being approved for the pretreatment Data Quality Objectives in support of high-level and low-level waste feed.

The highly radioactive nature of Hanford wastes makes the use of standard preservation techniques extremely difficult. In addition, the complexity of sampling, transporting, and storing of wastes makes some of the shorter holding times very difficult to achieve. For example, analyses performed on volatile and semivolatile organic compounds, many of which decompose and dissipate, have shorter holding times. Furthermore, the overall Tank Waste Remediation System information requirements are not predicated on strict compliance with regulatory requirements at this time. As the restoration and remediation of the Hanford Site continues, characterization for regulatory concerns will become more relevant. 


\subsection{SCREENING TECHNIQUES}

In addition to the analytical methods listed, there are a number of promising new screening techniques under development. If successfully applied, these techniques can reduce analytical turnaround times, reduce personal exposure, and increase the number of measurements performed. A partial listing of the available screening techniques is presented in Table 6-2.

\subsection{STATISTICAL ANALYSIS}

Once the chemical analyses are completed and concentration estimates have been obtained for the analytes at the various sampling locations, a statistical analysis of the data is needed for several reasons. For example, it will be useful for waste retrieval purposes to know whether the tank contents are uniform. Tank homogeneity is determined by comparing analyte concentrations between sampling locations using the analysis of variance (ANOVA). Another use is to determine whether the sample homogenization process is adequate. Samples are homogenized in an attempt to ensure that a given sample has uniform analyte concentrations throughout, and statistics are used to verify whether the homogenization process has accomplished this. Statistics can also estimate the sources of analytical error. Errors can occur at several different stages of a chemical analysis. Some of the sources of error are reagent contamination, poor instrument calibration, sample matrix interference, and analytical method error. By identifying a large source of error, steps may possibly be taken to account for the error while interpreting the data.

Table 6-2. Screening Techniques.

\begin{tabular}{|c|c|c|c|}
\hline Screening Technique & Application & Adyantages & Disadvantages \\
\hline $\begin{array}{l}\text { X-ray fluorescence } \\
\text { spectrometry. }\end{array}$ & $\begin{array}{l}\text { Nondestructive } \\
\text { screening for } \\
\text { metals. } \\
\text { Particularly useful } \\
\text { for determination } \\
\text { of "maximum" } \\
\text { values for RCRA } \\
\text { metals. }\end{array}$ & $\begin{array}{l}\text { Minimal sample } \\
\text { preparation } \\
\text { required, high } \\
\text { linear range. }\end{array}$ & $\begin{array}{l}\text { Sample radiation } \\
\text { sensitive. Very matrix } \\
\text { dependent. However, this } \\
\text { can be overcome through } \\
\text { the use of mathematical } \\
\text { corrections. }\end{array}$ \\
\hline Raman spectroscopy & $\begin{array}{l}\text { Determination of } \\
\text { molecular } \\
\text { species. }\end{array}$ & $\begin{array}{l}\text { Nondestructive, } \\
\text { minimal sample } \\
\text { preparation. }\end{array}$ & Subject to interferences. \\
\hline Long path UV/IR & $\begin{array}{c}\text { Organics in air. } \\
\text { - }\end{array}$ & $\begin{array}{l}\text { Instantaneous } \\
\text { analysis. }\end{array}$ & $\begin{array}{l}\text { Detection limit dependent } \\
\text { on path length. Benzene } \\
\text { by IR subject to water } \\
\text { interference. }\end{array}$ \\
\hline $\begin{array}{l}\text { Gamma-ray assay } \\
\text { methods }\end{array}$ & $\begin{array}{l}\text { Screening for } \\
\text { nuclear materials } \\
\text { such as }{ }^{137} \mathrm{Cs} .\end{array}$ & Nondestructive. & $\begin{array}{l}\text { Will not detect alpha } \\
\text { particles. }\end{array}$ \\
\hline Laser ablation ICP & Metals. & $\begin{array}{l}\text { Minimal sample } \\
\text { preparation. }\end{array}$ & $\begin{array}{l}\text { Only a very small portion } \\
\text { of sample is analyzed. }\end{array}$ \\
\hline
\end{tabular}




\subsection{CHARACTERIZATION DRIVERS}

\subsection{CHARACTERIZATION DATA USES}

Characterization of the wastes in the Hanford Site storage tanks is performed to assist in the resolution of safety issues; to allow for the safe storage of the wastes until retrieval operations begin; and to support the selection of waste removal, treatment, and final disposal options. . The Fiscal Year 1994 Tank Waste Remediation System Tank Waste Analysis Plan (Bell, 1994) lays out the sampling and analysis activities necessary for regulatory compliance and for safely storing, maintaining, treating, and disposing on site, or packaging for off-site disposal, all tank waste.

\subsubsection{Resolution of Safety Issues}

Concentration and inventory estimates derived from the characterization effort in the Tank Characterization Reports (TCRs) are used to address safety concerns associated with the tank wastes. The Defense Nuclear Facilities Safety Board has documented a series of safety concerns pertaining to the tank wastes (Conway, 1993); these concerns have driven the need to review the Unresolved Safety Questions and other safety issues dealing with the Hanford Site waste tanks and tank farms. Unresolved Safety Questions and ongoing safety issues that exist for the Hanford Site waste tanks include concerns over ferrocyanide $\left(\mathrm{Fe}(\mathrm{CN})_{6}^{-4}\right)$ content from previous cesium scavenging campaigns, organic layers or high total organics concentration, excessive heat generation from radioactive decay, flammable or noxious vapors in the tank headspace, and potential criticality in the bottom layers of waste sludges. The full range of available sampling and analysis methods are employed to provide data to address these safety issues. Information is also derived through reviews of the process and transfer history of each waste tank. From this review, waste simulants are sometimes developed and tested; waste simulants are considered to represent bounding conditions for the tank waste.

\subsubsection{Operations and Maintenance}

The analytical results from characterization provide concentration and inventory estimates that assist in the continued safe storage of the tank wastes and allow the continuation of current waste management operations. As a result of the design of the single-shell and double-shell tanks, design driven limits exist regarding several analytes (e.g., waste $\mathrm{pH}$, total heat generation, and the concentrations of nitrates, nitrites, and hydroxides). Waste compatibility determinations are necessary before tank-to-tank transfers, and prior to transfers to an evaporator. The volume of free liquid in the single-shell tanks is kept to a minimum. When possible, standing liquids are pumped off and/or interstitial liquids are salt-well pumped to the double-shell tanks in order to reduce the environmental release of wastes in the event of a single-shell tank leak. To conduct these and other intrusive pumping and transfer operations, previous knowledge of the tank contents and waste properties is required. 


\subsubsection{Facility and Process Design}

Since all wastes stored in the Hanford Site underground storage tanks will be retrieved, treated, vitrified, and then disposed of in monitored retrievable storage or geologic repositories, characterization estimates are required in order to design the retrieval equipment and the pretreatment, vitrification, and storage facilities. Retrieval planning and design requires information primarily about the physical properties of the waste, including viscosity, density, and critical flow rates. The pretreatment facilities are being designed to remove or separate species that hinder the operation of the vitrification operation or pose an environmental risk; therefore, minor waste constituents and trace elements are important. The design of the planned high-level and low-level vitrification plants is concerned primarily with the major waste constituents, which typically include the metals sodium, calcium, aluminum, and bismuth in abundance. The final waste form qualification for risk and performance assessment will focus on toxic metals and radionuclides. (Note that the initial round of tank characterization will not provide inventory estimates for permitting or closure of the tanks.)

\subsection{THE TRI-PARTY AGREEMENT}

At the Hanford Site, as well as at other DOE complexes, legally binding agreements have been made between the DOE and the applicable state and federal regulatory agencies. The Washington State Department of Ecology, the U.S. Environmental Protection Agency, and the DOE have entered into the Hanford Federal Facility Agreement and Consent Order, also known as the Tri-Party Agreement (Ecology et al., 1989; Ecology et al., 1993). The Tri-Party Agreement provides the framework, including both tasks and schedules, for the entire Site cleanup, and sets interim and final milestones necessary for the satisfactory completion of these tasks. Milestones have been set for mutually agreed-to deliverables that fulfill the letter and intent of the Tri-Party Agreement. Specifically, the Tri-Party Agreement establishes the criteria for final closure of the high-level waste tanks, for the transformation of the high-level and low-level wastes into forms that are satisfactory for final disposal, and for the eventual remediation and restoration of the Hanford Site. The Tri-Party Agreement milestone applying to the characterization of the Hanford Site waste tanks is Milestone $M-44$, which states in part:

Issue Tank Characterization Reports (TCRs) based on process knowledge, prior

- characterization data, and validated empirical data acquired after May 1989 for 177 Hanford high-level waste tanks.

\subsection{DATA QUALITY OBJECTIVE PROCESS}

The Data Quality Objective (DQO) process is an up-front strategic planning tool for designing the collection of the most representative environmental data in a cost-effective manner. This methodology directs the planners to define the precise problem, establish the desired action, identify the key variables that drive that action, and determine the cost of obtaining the minimum number of laboratory samples required. The resulting data quality objectives are quantitative and qualitative statements specified to ensure that data of known and appropriate quality are obtained during remedial investigation activities to support the selection of an appropriate remedial action alternative. The strategy for the phased implementation of the DQO planning process that supports all TWRS tank characterization needs is presented in The TWRS DQO Strategy. Table 7-1 lists the DQO efforts that apply to the characterization of the Hanford Site waste tanks. 
Table 7-1. Data Quality Objectives Documents.

\begin{tabular}{|l|c|}
\hline Tank Safety Screening & Document Number \\
\hline Ferrocyanide Safety Issue & WHC-SD-WM-SP-004 \\
\hline Flammable Gas Tanks Crust Bum Issue & WHC-SD-WM-DQO-003 \\
\hline Generic Tank Vapor Issue Resolution & WHC-SD-WM-DQO-002 \\
\hline Flammable Gas Tank & Not completed \\
\hline Waste Compatibility & WHC-SD-WM-DQO-001 \\
\hline Organic Fuel Rich Tank & WHC-SD-WM-DQO-006 \\
\hline Rotary Core Vapor Sampling & WHC-WD-WM-SP-003 \\
\hline Evaporator Operations & Not completed \\
\hline Process Control & Not completed \\
\hline Waste Tank Retrieval & Not completed \\
\hline Waste Tank Pretreatment & Not completed \\
\hline High-Level Waste Immobilization & Not completed \\
\hline Low-Level Waste Immobilization & Not completed \\
\hline Solid, Low-Level Waste Disposal & Not completed \\
\hline RCRA Part B Permit Application & Not completed \\
\hline Tank C-106 High-Heat Safety Issue & WHC-EP-0723 \\
\hline Organic Layer Sampling of Tank C-103 & PNL-8871 \\
\hline Tank C-103 Vapor and Gas Sampling & WHC-EP-0774 \\
\hline
\end{tabular}

The Washington State Department of Ecology has agreed upon a phased approach to Data Quality Objectives which will fulfill regulatory requirements. The long lead times required for design and construction of waste processing facilities make it necessary that the data needed for these activities be obtained as early as possible in order to expedite the removal of waste from the Hanford Site tanks. The phásed approach will allow acquisition of data for design purfoses that may not completely fulfill the regulatory requirements for disposal. Sampling and analysis protocols can be simplified until such time as the waste is in a more easily accessible form and location. This will not decrease the ultimate requirements for data quality, but will allow the delay of full implementation until the requirements can be met more easily and without unnecessarily encumbering the retrieval and treatment process. 
WHC-SD-WM-TI-648 REV 0

\subsection{TANK CHARACTERIZATION REPORT OVERVIEW}

The waste from single-shell tanks (SSTs) and double-shell tanks (DSTs) is sampled and analyzed according to the Tank Waste Remediation System Tank Waste Characterization Plan (Bell, 1993) and the more recent Fiscal Year 1994 Tank Waste Remediation System Tank Waste Analysis Plan (Bell, 1994). The Tank Characterization Report (TCR) summarizes the available information regarding the waste in a single- or double-shell tank, and arranges this information in a useful format for data users in various internal and external organizations. Information obtained from historical sources and synthetic waste studies' is compared to the actual waste measurements, and tank constituents, properties, and inventories are determined.

\subsection{TCR ORGANIZATION}

The following provides a brief overview of the contents of a Tank Characterization Report. This format was initially developed in De Lorenzo (1993), and refined in a series of Tank Characterization Reports (such as De Lorenzo et al., 1994).

\subsubsection{Executive Summary}

The Executive Summary is a concise overview of the most important information and conclusions of the Tank Characterization Report. It includes concentration and inventory estimate for the major chemicals and radionuclides present in the tank waste.

\subsubsection{Introduction}

TCR Section 1 presents the purpose and scope, and outlines any significant assumptions present in the report.

\subsubsection{Historical Tank Information}

TCR Section 2 begins with a description of the specific SST or DST being characterized. Figures showing basic tank design and typical configuration are included. Information is presented about tank design and history, process flows and waste streams, intertank transfers, any stabilization or isolation activities undertaken, and present status. Any suspected or confirmed tank-leakage data is also discussed.

\subsubsection{Tank Sampling Overview}

TCR Section 3 is intended to provide the reader with information about the most recent sampling event. A description of the sampler, the tank risers used and the criteria for their selection, and the numbering system for cataloging the samples are also included. The method of sampling is discussed, including whether push-mode or rotary-mode sampling was performed, the reason for that selection, and its impact on sample quality. Sample handling procedures, requested analyses, and tank farm sample numbers are also presented. 


\subsubsection{Sample Handling and Analytical Scheme}

The laboratory sample handling and breakdown process, the analyses performed on each portion of the waste, and the program goals met by the various analyses are described in TCR Section 4. Different suites of analyses are performed on segments, composites, drained liquids, and homogenization samples to support many activities and users. A description of the sample breakdown required to prepare the sample for the laboratory's analytical scheme is detailed in this section. Various tests and measurements are listed here as well, including homogenization tests, theological and physical measurements, and segment and core level analyses.

\subsubsection{Analytical Results and Waste Inventory}

Findings from the chemical, radiochemical, organic, and physical analyses are presented in TCR Section 5. A description of the methods employed, any difficulties encountered during analysis, and the results obtained for each sample are discussed. Measurements of physical properties such as shear strength, viscosity, particle size, percent water, $\mathrm{pH}$, and settling and thermal properties are covered. Graphs, tables, and figures are used as needed to present the data and sample results in the clearest manner possible.

\subsubsection{Analytical Results Interpretation}

To identify a tank's waste profile, the segment results for analytes distinct to the waste streams discharged to the tank are examined, and that information is combined with what is known regarding the tank's process history. The objective of TCR Section 6 is to develop a model for the spatial distribution of the compounds and radionuclides in the tank by relating analytical results with process history. The calculated bulk amounts of selected analytes are presented in this section, and their impact on potential safety concerns is discussed. Discrepancies are reconciled between historical/surveillance data and analytical results.

\subsubsection{Statistical Interpretation}

TCR Section 7 reports the results of a statistical analysis of the sample data. Homogenization of the samples is a critical step in the preparation of sample material. Therefore, the ability of the laboratory to homogenize solid core, segment, and subsegment samples is examined. Spatial variability, or the distribution of waste types within the tank, and the analytical error associated with the laboratory data are also discussed. Various constituent estimates and confidence intervals may be presented and discussed.

\subsubsection{Conclusions and Recommendations}

A summary of the results of the tank characterization effort is presented in TCR Section 8 , including major waste constituents and the agreement between data results and historical model predictions. Based on the data and analyses presented in the Tank Characterization Report and the goals of the characterization effort, recommendations are made concerning resolution of safety issues, further characterization needs, and impacts on future tank and sampling activities. To aid further characterization and retrieval activities, any conclusions with regard to tank groupings are made. 


\subsubsection{References}

Sources, documents, manuals, reports, studies, etc., used in the preparation of a TCR are listed in TCR Section 9.

\subsection{CONTEXT FOR INTERPRETATION OF SELECTED TANK ANALYTE VALUES}

The wastes in the tank farms vary substantially with regard to the degree of hazard posed. This section will provide an initial basis for qualitatively and quantitatively understanding characterization data, and will aid in making management decisions about the tank wastes: Relative comparisons of tank data values to established designations or limits of concem presented here can be made. A brief list of qualitative descriptions and quantitative parameters that should raise the level of concern about a tank are as follows:

- A tank with a watch list designation warrants substantially more consideration with regard to waste management and tank farm operations decisions than a nonwatch list tank. The criteria for watch list designation is established in Public Law 151510 and a brief description is provided in the glossary.

- Designation of waste in a tank as being complexed or noncomplexed has a substantial bearing on waste management decisions about the tank waste, especially any decisions .relating to waste volume reduction through the evaporator. The decision criterion for designation as complexed or noncomplexed is $10 \mathrm{~g}$ total organic carbon/L.

- A minimum percent moisture content of $17 \%$ for a waste phase has been established as a level of concern (Babad, 1994). There is some amount of moisture associated with nearly all of the wastes in the tanks; however, the moisture content varies widely. between waste phases. Liquid wastes are generally greater than $90 \%$ water. Sludges and slurries have been observed to range widely, between 20 and $80 \%$ water. Crusts and salt cakes usually have a moisture content less than $20 \%$.

- The fuel energy value of a waste as determined by differential scanning calorimetry is an extremely important value. Exotherm values in excess of -125 $\mathrm{cal} / \mathrm{dry}$ gram of waste have been established as the decision criterion for further analytical investigation and potential watch list designation (Babad, 1994).

- Related to both the fuel energy value criterion and complexed/noncomplexed waste designation is the total organic carbon content of the waste. Total organic carbon generally provides the fuel driving the reaction observed in DSC measurements. A total organic carbon content value of 5 weight percent (dry basis) has been established as the safety criterion (Babad, 1994). However, as related above, total organic carbon values for making waste management decisions about liquid or slurry wastes are much lower, $10 \mathrm{~g} / \mathrm{L}$.

Total alpha concentrations of $1 \mathrm{~g}{ }^{239} \mathrm{Pu} / \mathrm{L}$ waste has been established as the level of concern for criticality (Babad, 1994). 


\subsection{TANK SYSTEM DANGEROUS WASTE PERMIT}

This section describes the purpose of the Double- and Single-Shell Tank System Dangerous Waste Permit Applications (DOE-RL, 1993a; DOE-RL, 1993b) which address the storage and treatment of mixed waste - waste that is both dangerous, as determined by the Washington State Department of Ecology "Dangerous Waste Regulations" (Ecology, 1991), and radioactive waste.

The general purposes of this regulation are the following: 1) Designate those solid wastes which are dangerous or extremely hazardous to the public health and environment. 2) Provide for surveillance and monitoring of dangerous and extremely hazardous wastes until they are detoxified, reclaimed, neutralized, or disposed of safely. 3) Provide the form and rules necessary to establish a system for manifesting, tracking, reporting, monitoring, recordkeeping, sampling, and labeling dangerous and extremely hazardous wastes. 4) Establish the siting, design, operation, closure, post-closure, financial, and monitoring requirements for dangerous and extremely hazardous waste transfer, treatment, storage, and disposal facilities. 5) Establish design, operation, and monitoring requirements for managing the State's extremely hazardous waste disposal facility. 6) Establish and administer a program for permitting dangerous and extremely hazardous waste management facilities. 7) Encourage recycling, reuse, reclamation, and recovery to the maximum extent possible (Ecology, 1991).

\subsubsection{Waste Characteristics}

Waste characteristics are divided into four main subcategories: ignitability, corrosivity, reactivity, and toxicity. A solid waste that exhibits the characteristic of ignitability (as set by the Washington Administrative Code [WAC]) shall be designated a dangerous waste (DW), and shall be assigned the dangerous waste number of D001 (see Table 8-1). Likewise, solid wastes that exhibit the characteristic of corrosivity and reactivity shall be designated a DW, and assigned the dangerous waste number $\mathrm{DOO} 2$ and $\mathrm{D} 003$, respectively. A solid waste exhibits the toxicity characteristic if, using the Toxicity Characteristic Leaching Procedure (EPA, 1990), it contains any of the contaminants listed in Table 8-1 at concentrations equal to or greater than the respective value given in the list. Two levels of concentration are established for the contaminants listed. Any waste containing one or more contaminants with concentrations at or above the extremely hazardous waste (EHW) threshold shall cause that waste to be designated EHW. Any waste containing contaminants which occur at concentrations at or above the DW threshold only shall be designated DW.

\subsubsection{Waste Criteria}

The dangerous waste criteria consist of three subcategories: toxic dangerous wastes, persistent dangerous wastes, and carcinogenic dangerous wastes. Several criteria are listed in the WAC to determine if waste is toxic dangerous waste and will not be explained here. A waste designated as DW by toxicity criteria shall be assigned the dangerous waste number of WT02 (see Table 8-2). A waste designated as EHW by toxicity criteria shall be assigned the dangerous waste number of WT01. As defined in the WAC (173-303-040) for persistent dangerous wastes, persistent constituents are chemical compounds which are either halogenated hydrocarbons, or polycyclic aromatic hydrocarbons. Also, a carcinogenic substance is a substance which has sufficient or limited evidence as a human or animal carcinogen as listed in The National Institute 
Table 8-1. Dangerous Waste Characteristics.

(sheet 1 of 3 )

\begin{tabular}{|c|c|c|c|c|}
\hline \multicolumn{5}{|c|}{ 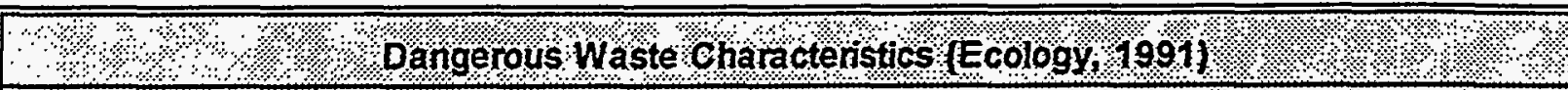 } \\
\hline $\begin{array}{l}\text { Dangerous } \\
\text { Waste Number }\end{array}$ & ২+. & . & plion $1 .+4.4 .4$ & (?. \\
\hline D001 & \multicolumn{4}{|c|}{$\begin{array}{l}\text { A solid waste that exhibits the characteristic of ignitability (WAC 173- } \\
303-090[5]) \text {. }\end{array}$} \\
\hline D002 & \multicolumn{4}{|c|}{$\begin{array}{l}\text { A solid waste that exhibits the characteristic of corrosivity (WAC 173- } \\
303-090[6]) \text {. }\end{array}$} \\
\hline D003 & \multicolumn{4}{|c|}{$\begin{array}{l}\text { A solid waste that exhibits the characteristic of reactivity (WAC 173- } \\
303-090[7]) \text {. }\end{array}$} \\
\hline \multicolumn{5}{|c|}{ 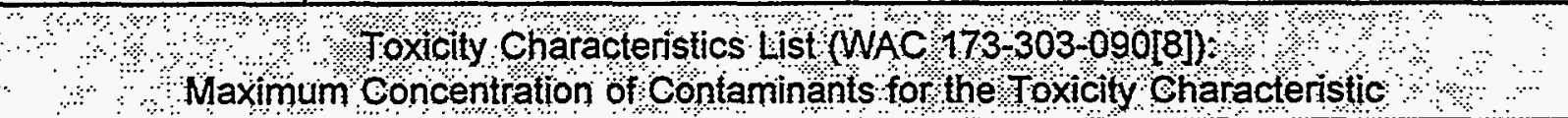 } \\
\hline $\begin{array}{l}\text { Bangerous } \\
\text { Waste Number } \\
\text { }\end{array}$ & Contaminant & $\begin{array}{l}\text { Cehemical } \\
\text { Abstracts } \\
\text { Services } \\
\text { Number }\end{array}$ & $\begin{array}{l}\text { Extremely } \\
\text { Hazardous } \\
\text { Waste (EllW } \\
\text { (mg } 1 \mathrm{l})\end{array}$ & $\begin{array}{l}\text { Dangerous } \\
\text { Waste (DW) } \\
\text { (mgll) }\end{array}$ \\
\hline D004 & Arsenic & $(7440-38-2)$ & 500 & 5.0 \\
\hline D005 & Barium & $(7440-39-3)$ & 10,000 & 100.0 \\
\hline D018 & Benzene & $(71-43-2)$ & 50 & 0.5 \\
\hline D006 & Cadmium & $(7440-43-9)$ & 100 & 1.0 \\
\hline D019 & $\begin{array}{l}\text { Carbon } \\
\text { tetrachloride }\end{array}$ & $(56-23-5)$ & 50 & 0.5 \\
\hline D020 & Chlordane & $(57-74-9)$ & 3.0 & 0.03 \\
\hline$D 021$ & Chlorobenzene & $(108-90-7)$ & 10,000 & 100.0 \\
\hline D022 & Chloroform & $(67-66-3)$ & 600 & 6.0 \\
\hline D007 & Chromium & $(7440-47-3)$ & 500 & 5.0 \\
\hline D023 & o-Cresol & $(95-48-7)^{9}$ & 20,000 & 200 \\
\hline D024 & m-Cresol & $(108-39-4)^{1}$ & 20,000 & 200 \\
\hline D025 & p-Cresol & $(106-44-5)^{1}$ & 20,000 . & 200 \\
\hline D026 & Cresol & (see footnote 1 ) & 200 & - \\
\hline D016 & $2,4-D$ & $(94-75-7)$ & 1,000 & 10.0 \\
\hline D027 & $\begin{array}{l}\text { 1,4-Dichloro- } \\
\text { benzene }\end{array}$ & $(106-46-7)$ & 750 & 7.5 \\
\hline
\end{tabular}


Table 8-1. Dangerous Waste Characteristics.

(sheet 2 of 3 )

\begin{tabular}{|c|c|c|c|c|}
\hline $\begin{array}{l}\text { Dangerous } \\
\text { Waste Number }\end{array}$ & ocontaminant & $\begin{array}{l}\text { \&ehemical } \\
\text { Abstracts } \\
\text { Services } \\
\text { Number }\end{array}$ & $\begin{array}{l}\text { Extremely } \\
\text { Hazardous } \\
\text { Waste }(E H W) \\
\text { (mgl })\end{array}$ & $\begin{array}{l}\text { Pangerous } \\
\text { Waste fow } \\
\text { fing/( })\end{array}$ \\
\hline D028 & $\begin{array}{l}\text { 1,2-Dichloro- } \\
\text { ethane }\end{array}$ & $(107-06-2)$ & 50 & 0.5 \\
\hline$D 029$ & $\begin{array}{l}\text { 1,1-Dichloro- } \\
\text { ethylene }\end{array}$ & $(75-35-4)$ & 70 & 0.7 \\
\hline D030 & $\begin{array}{l}\text { 2,4-Dinitro- } \\
\text { toluene }\end{array}$ & $(121-14-2)^{2}$ & 13 & 0.13 \\
\hline D012 & Endrin & $(72-20-8)$ & 2 & 0.02 \\
\hline$D 031$ & $\begin{array}{l}\text { Heptachlor } \\
\text { (and its } \\
\text { epoxide) }\end{array}$ & $\begin{array}{c}(76-44-8) \\
.\end{array}$ & 0.8 & 0.008 \\
\hline D032 & $\begin{array}{l}\text { Hexachloro- } \\
\text { benzene }\end{array}$ & $(118-74-1)^{2}$ & 13 & 0.13 \\
\hline D033 & $\begin{array}{l}\text { Hexachloro- } \\
\text { butadiene }\end{array}$ & $(87-68-3)$ & 50 & 0.5 \\
\hline D034 & $\begin{array}{l}\text { Hexachloro- } \\
\text { ethane }\end{array}$ & $(67-72-1)$ & 300 & 3.0 \\
\hline D008 & Lead & $(7439-92-1)$ & 500 & 5.0 \\
\hline D013 & Lindane & $(58-89-9)$ & 40 & 0.4 \\
\hline Doog & Mercury & $(7439-97-6)$ & 20 & 0.2 \\
\hline Do14 & Methoxychlor & $(72-43-5)$ & 1,000 & 10.0 \\
\hline D035 & $\begin{array}{l}\text { Methyl ethyl } \\
\text { ketone }\end{array}$ & $(78-93-3)$ & 20,000 & 200.0 \\
\hline D036 & Nitrobenzene & $(98-95-3)$ & 200 & 2.0 \\
\hline D037 & $\begin{array}{l}\text { Pentachloro- } \\
\text { phenol }\end{array}$ & $(87-86-5)$ & 10,000 & 100.0 \\
\hline D038 & Pyridine & $(110-86-1)^{2}$ & 500 & 5.0 \\
\hline D010 & Selenium & $(7782-49-2)$ & 100 & 1.0 \\
\hline D011 & Silver & $(7440-22-4)$ & 500 & 5.0 \\
\hline D039 & $\begin{array}{l}\text { Tetrachloro- } \\
\text { ethylene }\end{array}$ & $(127-18-4)$ & 70 & 0.7 \\
\hline
\end{tabular}


Table 8-1. Dangerous Waste Characteristics.

(sheet 3 of 3)

\begin{tabular}{|c|c|c|c|c|}
\hline $\begin{array}{l}\text { bangerous } \\
\text { Waste Number } \\
\text { । }\end{array}$ & Contaminant & $\begin{array}{l}\text { Chemical } \\
\text { Abstracts } \\
\text { Senvices } \\
\text { Number }\end{array}$ & $\begin{array}{l}\text { Extremely } \\
\text { Hazardous } \\
\text { Waste }(\mathrm{EHHW}) \\
\text { (mglt) }\end{array}$ & $\begin{array}{l}\text { Dangerous } \\
\text { Waste (Duv) } \\
\text { (milu })\end{array}$ \\
\hline D015 & Toxaphene & $(8001-35-2)$ & 50 & 0.5 \\
\hline D040. & $\begin{array}{l}\text { Trichloro- } \\
\text { ethylene }\end{array}$ & $(79-01-6)$ & 50 & 0.5 \\
\hline D041 & $\begin{array}{l}\text { 2,4,5-Trichloro- } \\
\text { phenol }\end{array}$ & $(95-95-4)$ & 40,000 & 400.0 \\
\hline D042 & $\begin{array}{l}2,4,6-\text {-Trichloro- } \\
\text { phenol }\end{array}$ & $(88-06-2)$ & 200 & 2.0 \\
\hline D017 & $\begin{array}{l}2,4,5-T P \\
\text { (Silvex) }\end{array}$ & $(93-72-1)$ & 100 & 1.0 \\
\hline D043 & Vinyl chloride & $(75-01-4)$ & 20 & 0.2 \\
\hline
\end{tabular}

'If $0-, \mathrm{m}-;$ and $\mathrm{p}$-Cresol concentrations cannot be differentiated, the total Cresol (D026) concentration is used. The dangerous waste level for total Cresol is $200 \mathrm{mg} / \mathrm{L}$ and the extremely hazardous waste level for total cresol is $20,000 \mathrm{mg} / \mathrm{L}$.

${ }^{2}$ Quantitation limit is greater than the calculated regulatory level. The quantitation limit therefore becomes the regulatory level.

for Occupational Safety and Health Registry of Toxic Effects of Chemical Substances and the Integrated Risk Information System or Health Effects Assessment Summary Table. A solid waste that contains one or more carcinogenic substances is designated DW and assigned a waste number of WC02 if the concentration of any one carcinogenic substance exceeds $0.01 \%$ of the waste.quantity, or the total concentration summed for all carcinogenic substances exceeds $1.0 \%$ of the waste quantity.

\subsubsection{Waste Sources}

A list of dangerous waste sources appears in WAC 173-303-9904. Any waste that is listed in the dangerous waste sources list is designated as a dangerous waste, and is identified as DW, except for a few specific cases the WAC designates as extremely hazardous waste (EHW). Wastes that are pertinent to the Tank Characterization Reports are listed in Table 8-3. Many other wastes are listed in the WAC, but this section is not aimed to be all intensive. 
Table 8-2. Waste Criteria.

\begin{tabular}{|c|c|c|}
\hline \multicolumn{3}{|c|}{ Geneno Dangerous Waste Numbers $(W A C-173-303-100)}$, \\
\hline $\begin{array}{l}\text { Dangerous Waste } \\
\text { Number }\end{array}$ & $\begin{array}{l}\text { Dangerous Waste } \\
\text { Enteria and } \\
\text { Besignation }\end{array}$ & $\begin{array}{lll}\text { Criteria } \\
\text { + }\end{array}$ \\
\hline \multicolumn{3}{|c|}{ Toxic Dangerous Wastes } \\
\hline WT01 & $\begin{array}{l}\text { Extremely } \\
\text { Hazardous Waste } \\
\text { (EHW) }\end{array}$ & $\begin{array}{l}\text { Depends on constituent concentration and } \\
\text { quantity (WAC-173-303-100 [s]) }\end{array}$ \\
\hline WT02 & $\begin{array}{l}\text { Dangerous Waste } \\
\text { (DW) }\end{array}$ & $\begin{array}{l}\text { Depends on constituent concentration and } \\
\text { quantity (WAC-173-303-100 [s]) }\end{array}$ \\
\hline \multicolumn{3}{|c|}{ Persistent Dangerous Wastes } \\
\hline WP01 & $\begin{array}{l}\text { Extremely } \\
\text { Hazardous Waste } \\
\text { (EHW) }\end{array}$ & $\begin{array}{l}\text { Halogenated Hydrocarbons concentration } \\
\text { greater than } 1.0 \%\end{array}$ \\
\hline WPO2 & $\begin{array}{l}\text { Dangerous Waste } \\
\text { (DW) }\end{array}$ & $\begin{array}{l}\text { Halogenated Hydrocarbons concentration } \\
0.01 \% \text { to } 1.0 \%\end{array}$ \\
\hline WP03 & $\begin{array}{l}\text { Extremely } \\
\text { Hazardous Waste } \\
\text { (EHW) }\end{array}$ & $\begin{array}{l}\text { Polycyclic Aromatic Hydrocarbons } \\
\text { concentration greater than } 1.0 \%{ }^{\star}\end{array}$ \\
\hline \multicolumn{3}{|c|}{ Carcinogenic Dangerous Wastes } \\
\hline WC01 & $\begin{array}{l}\text { Extremely } \\
\text { Hazardous Waste } \\
\text { (EHW) }\end{array}$ & $\begin{array}{l}\text { Waste exhibiting carcinogenic compounds } \\
\text { in concentrations exceeding those } \\
\text { specified in WCO } 2 \text { designated waste. }\end{array}$ \\
\hline WC02 & $\begin{array}{l}\text { Dangerous Waste } \\
\text { (DW) }\end{array}$ & $\begin{array}{l}\text { Concentration of any one carcinogenic } \\
\text { substance exceeds } 0.01 \% \text { of the waste } \\
\text { quantity; or the total concentration summed } \\
\text { for all carcinogenic substances exceeds } \\
1.0 \% \text { of the waste quantity }\end{array}$ \\
\hline
\end{tabular}

-No DW concentration level for Polycyclic Aromatic Hydrocarbons. 
Table 8-3. Waste Sources.

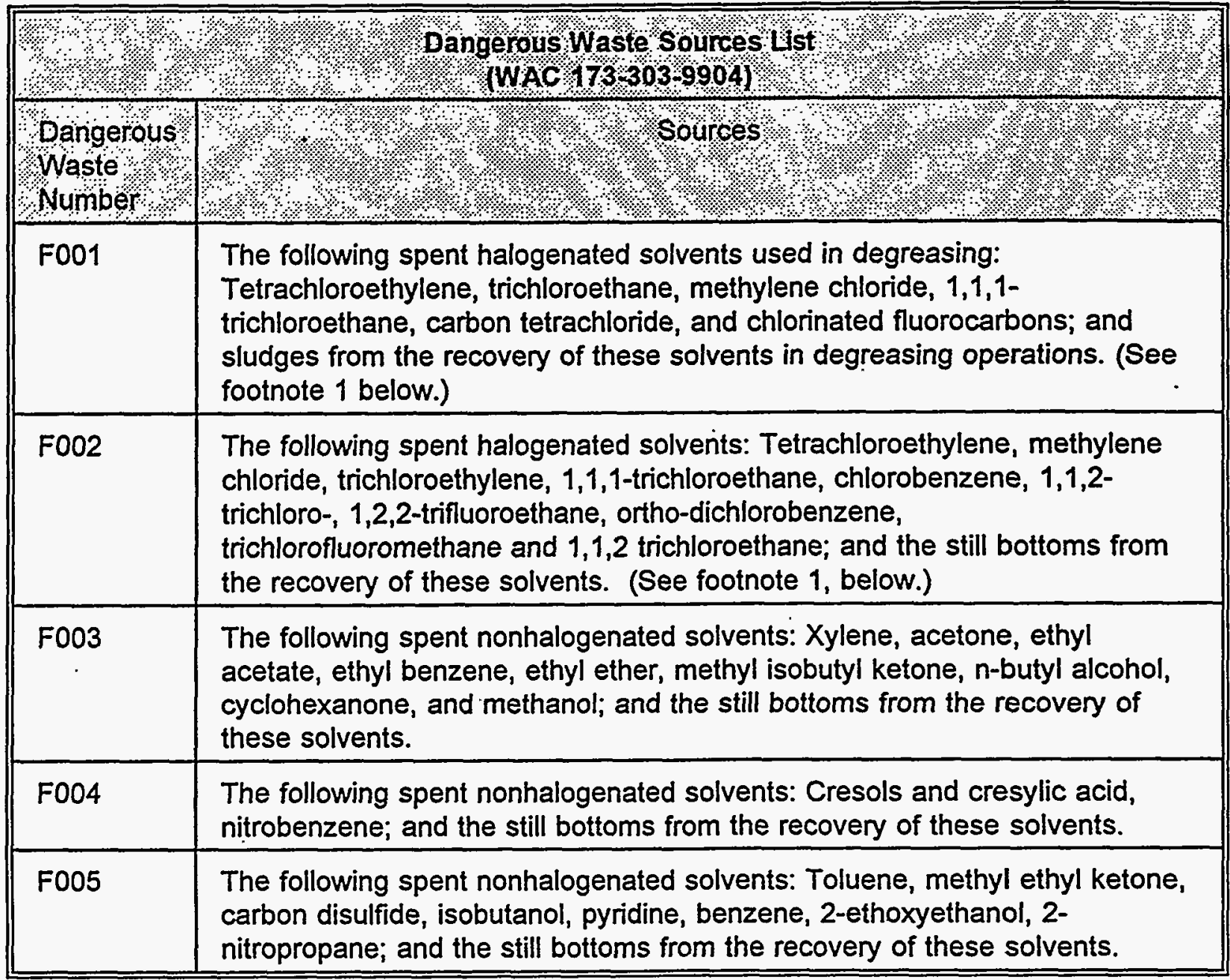

'These wastes contain or may contain halogenated hydrocarbons. Although WAC 173-303-082 states that these wastes are dengerous waste (DW), WAC 173-303-070(5), special knowledge, requires generators who know that their waste contains greater than $1 \%$ of these listed halogenated hydrocarbons to designate their waste extremely hazardous waste (EHW). 


\subsection{HISTORICAL TANK ESTIMATES}

Historical information supplements data gathered from in-tank sampling activities and provides an important context for interpreting analytical results. The amount of historical data, while imposing, is rather narrowly defined. Data in the form of process knowledge, effluent discharges, waste tank transfers, and evaporator campaigns form an initial basis for review. Physical and chemical characterization information, as well as detailed surveillance records, are also available. This information can be coupled with several general assumptions regarding the physical behavior of the slurries discharged to the tanks, and the ongoing processes occurring inside the tanks to allow a detailed estimation of the contents of each waste tank.

Several previous and ongoing efforts to compile and present this historical information have been undertaken. In each case, the projects have sought to arrive at waste tank inventory estimates independent of laboratory generated analytical data results. These historical estimates are contained in a series of documents, computer models, and characterization databases.

- TRAC - The Track Radioactive Constituents (TRAC) computer program was the first detailed attempt to quantitatively predict the inventories of the Hanford Site single-shell tanks. The TRAC program estimated waste inventories based on nuclear fuels production models, reprocessing and waste management flowsheets, tank transfers, and radioactive decay calculations. Input to the model consisted of ordered, quarterly transaction information and estimated waste compositions for the various waste streams. A series of subroutines then processed the input data according to predicted constituent solubilities, decay models (the ORIGEN2 code), and solid/liquid partitions. The output consists of the approximate inventories of 65 radionuclides and 30 nonradioactive chemical constituents in each of the 149 single-shell tanks as of the first quarter of 1981.

Validation of the codes used in the TRAC Model has not been performed, and there are severe limitations in some of the initial assumptions, waste compositions, and solubilities. For these reasons, the conclusions and model outputs should be regarded as approximations at best. In the absence of more conclusive or higher quality data, the results and estimates of the TRAC model are often used as a preliminary approximation for expected tank contents and inventory. A detailed description of the TRAC system can be found in TRAC: Preliminary Estimation of the Waste Inventories in Hanford Tanks Through 1980 (Jungfleisch, 1984).

- Estimated Chemical and Radiochemical Inventory Spreadsheets - Detailed inventories for all Hanford Site waste tanks are being estimated through a series of spreadsheet based computer models by Lós Alamos National Laboratory (LANL). As part of their characterization effort, LANL is deriving detailed composition estimates for the various waste streams sent to the Hanford Site tank farms. By coupling this data with spreadsheet-based transaction records, an accounting of the waste present in each tank, as a function of time, is produced. Initial indications are that these model estimates, in their current form, are moderately successful in predicting certain bulk waste properties and inventories. Further verification of the computer model and cross-checking of data to other historical sources is continuing at this time. It is possible that these estimates will prove to be an adequate predictive model for the major metallic waste constituents. As more comparisons between the historical estimates and the TCR results are made, further conclusions about the utility and defensibility of the characterization information contained 
in the inventory spreadsheets can be made. Additional background on the LANL characterization effort is contained in the Estimated Chemical and Radiochemical Inventories Spreadsheet: NE Quadrant, $A, A X, B, B X, B Y, C$ Farms (Agnew, 1994b).

- Historical Tank Content Estimate Reports - Historical evaluations of all Hanford Site underground waste storage tanks are being performed by ICF Kaiser Hanford Company. These evaluations are presented in a series of reports, grouped by approximate geographic division into four quadrants of the 200 East and 200 West Areas. Each Historic Tank Content Estimate report, and its associated supporting documents, contains quadrant specific waste transfer and level data, tank physical information, temperature data, past sampling results, and drywell and liquid observation well data. Also presented in the reports are the inventory predictions generated by LANL. Further information about the historical data gathering effort can be found in the Historical Tank Content Estimate for the Northeast Quadrant of the Hanford 200 East Areas (Brevick et al., 1994a) and in the Historical Tank Content Estimate for the Southwest Quadrant of the Hanford 200 West Areas (Brevick et al., 1994b). 


\subsection{REFERENCES}

Agnew, S. F., P. Baca, R. Corbin, and J. Gustafson, 1993, JAAWS-1 Spreadsheet (beta release) Hanford Glossary, Los Alamos National Laboratory, Los Alamos, NM.

Agnew, S.F., 1994a, Hanford Defined Wastes: Chemical and Radjonuclide Compositions, Westinghouse Hanford Company, Richland, WA.

Agnew, S. F., 1994b, Estimated Chemical and Radiochemical Inventories Spreadsheet: NE Quadrant, $A, A X, B, B X, B Y, C$ Farms, WHC-SD-WM-TI-628, Rev. 0, Westinghouse Hanford Company, Richland, WA.

Alstad, A. T., 1992, Riser Configuration Document for Single-Shell Waste Tanks, WHC-SD-RE-TI053, Rev. 8, Westinghouse Hanford Company, Richland, WA.

Anderson, J. D., 1990, A History of the 200 Area Tank Farms, WHC-MR-0132, Westinghouse Hanford Company, Richland, WA.

ASTM, 1973, Weighted Bottle Sample Method, ASTM E-300-73, American Society for Testing and Materials, Philadelphia, PA.

Ballinger, M.Y., and R.B. Hall, 1991, A History of Major Hanford Facilities and Process Involving Radioactive Material, PNL-6964-HEDR, Pacific Northwest Laboratory, Richland, WA.

Bell, K.E., 1993, Tank Waste Remediation System Tank Waste Characterization Plan, WHC-SDWM-PLN-047, Rev. 0, Westinghouse Hanford Company, Richland, WA.

Bell, K. E., 1994, Fiscal Year 1994 Tank Waste Remediation System Tank Waste Analysis Plan. WHC-SD-WM-PLN-077, Rev.0, Westinghouse Hanford Company, Richland, WA.

Bergmann, L.M., 1990, Single-Shell Tank Isolation Safety Analysis Report, WHC-SD-WM-006, Rev 2., Westinghouse Hanford Company, Richland, WA.

Boomer, K.D., S.K. Baker, A.L. Boldt, M.D. Britton, L.E. Engelsman, J.D. Galbraith, J.S. Garfield, K.A. Giese, C.E., Golberg, B.A. Higley, K.J. Hull, L.J. Johnson, R.P. Knight, J.S. Layman, R.S. Marusich, R.J. Parazin, M.G. Piepho, G. W. Reddick, E.J. Slaathaug, T.L. Waido, and C.E. Worcester, 1991, Systems Engineering Study for the Closure of Single-Shell Tanks, WHC-EP-0405, Draft A, Volume 1, Westinghouse Hanford Company, Richland, WA.

Boomer, K.D., S.K. Baker, A.L. Boldt, M.D. Britton, J.D. Galbraith, J.S. Garfield, C.E., Golberg, B.A. Higley, L.J. Johnson, M.J. Kupfer, R.S. Marusich, R.J. Parazin, M.G. Piepho, A.N. Praga, G. W. Reddick, E.J. Slaathaug, L.M. Swanson, T.L. Waldo, and C.E. Worcester, 1992, Tank Waste Systems Engineering Study, WHC-EP-0405, Rev. 0, Westinghouse Hanford Company, Richland, WA. 
Borsheim, G.L., and B.C. Simpson, 1991, An Assessment of the Inventories of the Ferrocyanide Watchlist Tanks, WHC-SD-WM-ER-133, Rev. 0, Westinghouse Hanford Company, Richland, WA.

Brager, H.R., 1993, Summary Information on Flammable Gas Watch List Tanks, WHC-SD-WMTI-584, Westinghouse Hanford Company, Richland, WA.

Brevick, C.H., L.A. Gaddis, and W.W. Pickett, 1994a, Historical Tank Content Estimate for the Northeast Quadrant of the Hanford 200 East Areas, WHC-SD-WM-ER-349, ICF Kaiser Hanford Company, Richland, WA.

Brevick, C.H., L.A. Gaddis, and W.W. Pickett, 1994b, Historical Tank Content Estimate for the Southwest Quadrant of the Hanford 200 West Area, WHC-SD-WM-ER-352, ICF Kaiser Hanford Company, Richland, WA.

"Comprehensive Environmental, Response, Compensation, and Liability Act," 42 U.S.C § 9601 , et seq.

Conway, J. T., 1993, DNFSB Recommendation 93-5 to the Secretary of Energy, (Letter 9400070 to H. R. O'Leary, DOE, July 19), Defense Nuclear Facilities Safety Board, Washington, D.C.

De Lorenzo, D. S., 1993, Tank Characterization Report (TCR) Procedure (Letter WHC9400198 to T.H. Dove, Westinghouse Hanford Company, September 30, 1993), Los Alamos Technical Associates, Richland, WA.

De Lorenzo, D. S., A. T. DiCenso, L. C. Amato, D. B. Hiller, G. L. Nuttall, and B. C. Simpson, 1994, Tank Characterization Report for Double-Shell Tank 241-AP-106, WHC-SD-WMER-361, Westinghouse Hanford Company, Richland, WA.

De Lorenzo, D. S., and B. C. Simpson, 1994, Hanford Site Waste Tank Characterization, WHCSA-2584-FP, Westinghouse Hanford Company, Richland, WA.

DOE-RL, 1993a, Single-Shell Tank System Dangerous Waste Permit Application, DOE-RL-88-21, U.S. Department of Energy Richland Operations Office, Richland, WA.

DOE-RL, 1993b, Double-Shell Tank System Dangerous Waste Permit Application, DOE-RL-9039, U.S. Department of Energy Richland Operations Office, Richland, WA.

Ecology, 1991, "Dangerous Wastée Regulations," WAC 173-303, Washington State Department of Ecology, Olympia, WA.

Ecology, EPA, and DOE, 1989, Hanford Federal Facility Agreement and Consent Order, Washington State Department of Ecology, Olympia, WA.

Ecology, EPA, and DOE, 1993, Hanford Federal Facility Agreement and Consent Order, as amended, Washington State Department of Ecology, Olympia, WA. 
Edwards, J.N., 1993, Safety Assessment for Proposed Pump Operations to Mitigate Episodic Gas Release in Tank 241-SY-101, LAUR-92-3196, Los Alamos National Laboratory, Los Alamos, NM.

EPA, 1989, USEPA Contract Laboratory Program Statement of Work for Inorganic Analyses, U.S. Environmental Protection Agency, Washington, D.C..

EPA, 1990, Identification and Listing of Hazardous Wastes, 40 CFR 261.

EPA, 1992, Test Methods for Evaluating Solid Waste (SW-846), Third Edition, Final Update 1, U.S. Environmental Protection Agency, Washington, D.C.

Gerber, M. S., 1992, Legend and Legacy: Fifty Years of Defense Production at the Hanford Site, WHC-MR-0293, Rev. 1, Westinghouse Hanford Company, Richland, WA.

Gerber, M. S., 1993, The Hanford Site: An Anthology of Early Histories, WHC-MR-0435, Westinghouse Hanford Company, Richland, WA.

Gerber, M. S., 1994, "PAC-MEN" of the Hanford Site Await Decommissioning, Westinghouse Hanford Company, Richland, WA.

Grigsby, J.M., D.B. Bechtold, G.L. Borsheim, M.D. Crippen, D.R. Dickinson, G.L. Fox, D.W. Jeppson, M. Kummerer, J.M. McLaren, J.D. McCormack, A. Padilla, B.C. Simpson, and D.D Stepnewski, 1992, Ferrocyanide Waste Tank Hazard Assessment-Interim Report, WHC-SD-WM-RPT-032, Rev. 1, Westinghouse Hanford Company, Richland, WA.

Hanlon, B. M., 1994, Tank Farm Surveillance and Waste Status Summary Report For March 1994, WHC-EP-0182-72, Westinghouse Hanford Company, Richland, WA.

Husa, E. I., R. E. Raymond, R. K. Welty, S. M. Griffith, B. M. Hanlon, R. R. Rios, N. J. Vermeulen, 1993, Hanford Site Waste Storage Tank Information Notebook, WHC-EP. 0625, Westinghouse Hanford Company, Richland, WA.

Jones; T.E., S.G. McKinley, J.M. Tingey, T.M. Longaker, J.A. Gibson, 1991, Single-Shell Tank Waste Characterization Project, Pacific Northwest Laboratory, Richland, WA.

Jungfleisch, F. M., 1983, Supplementary Information for the Preliminary Estimation of Waste Tank Inventories in Hanford Tanks through 1980, SD-WM-TI-058, Rockwell Hanford Operations, Richland, WA.

Jungfleisch, F. M., 1984, Preliminary Estimation of the Waste Inventories in Hanford Tanks through 1980, SD-WM-TI-057, Rev. 0, Rockwell Hanford Operations, Richland, WA.

Koerna, J. A., 1986, 241-AP Tank Farm Facility Description Manual, FDM-T-270-00001, Rockwell Hanford Operations, Richland, WA.

'Koontz, R. L., 1989, Double-Shell Tank Farm Facility Safety Analysis Report, WHC-SD-WM-SAR016 R1, Westinghouse Hanford Company, Richland, WA. 
Koreski, G. M., 1994, Waste Volume Projections: Thermocouple and Surface Level Readings, Westinghouse Hanford Company, Richland, WA.

Long, J.T., 1967, Engineering for Nuclear Fuel Reprocessing, Gordon and Beach Science Publishers Inc., New York, NY.

"Resource Conservation and Recovery Act," 42 U.S.C. § 6901-6902 (1976).

Simpson, B. C. and G. L. Borsheim, 1993, Tank Characterization Data Report: 241-C-109, WHCEP-0688, Westinghouse Hanford Company, Richland, WA.

Simpson, B.C., G.L. Borsheim, and L. Jensen, 1993, Tank Characterization Report: Tank 241-C109, WHC-EP-0668, Westinghouse Hanford Company, Richland ,WA.

Smith, D. A., 1991, Single-Shell Tank Isolation Safety Analysis Report, SD-WM-SAR-006 R2, Rockwell Hanford Operations, Richland, WA.

Welty, R.K., 1988, Waste Storage Tank Status and Leak Detection Criteria, WHC-SD-WM-TI-356, Westinghouse Hanford Company, Richland, WA.

WHC, 1993, Hanford Site Tank Farms Facilities Interim Safety Basis, WHC-SD-WM-ISB-001, Vol. 2, Rev.0, Westinghouse Hanford Company, Richland, WA.

Winters, W. I., L. Jensen, L. M. Sasaki, R. L. Weiss, J. F. Keller, A. J. Schmidt, and M. G. Woodruff, 1990, Waste Characterization Plan for Hanford Site Single-Shell Tanks, WHCEP-0210, Westinghouse Hanford Company, Richland, WA.

U.S. Department of Energy, 1987, First Environmental Impact Statement Disposal of Hanford Defense High-Level, Transuranic and Tank Wastes, DOE/EIS-0113, Volume 2, DOE-RL, Richland, WA. 
WHC-SD-WM-TI-648 REV 0

Appendix A. Glossary 


\section{Glossary}

This glossary was compiled from information found in Agnew et al. (1993), Anderson (1990), Brevick et al. (1994a and 1994b), and Jungfleisch (1984).

$1 \mathrm{C}$

1st Generation Tank

$2 \mathrm{C}$

2nd Generation Tank

$221-B$

221-T

224-F

242-A

242-B

242-S

242- $\mathrm{T}$

3rd Generation Tank
First-cycle decontamination waste from the bismuth phosphate process. Often included cladding waste. Held $10 \%$ of the fission products and $1 \%$ of plutonium.

The original tank design used for the $B, C, T, U$ (excluding 200 series tanks), and BX Tank Farms. These tanks have an operating capacity of $2,010 \mathrm{~kL}(530,000$ gal), a diameter of $22.8 \mathrm{~m}(75 \mathrm{ft})$, a 30-cm (12-in.) dish bottom, and a 1.2-m (4-ft) knuckle.

Second-cycle decontamination waste from the bismuth phosphate process. Supernatant was often cribbed. Contained $0.1 \%$ of the fission products and $1 \%$ of the plutonium.

A design that was the same as the original (1st generation) design, except the operating capacity was increased to $2,870 \mathrm{~kL}(758,000$ gal).

B Plant used for bismuth phosphate processing, 1944-52, then for fission products recovery.

T Plant used for bismuth phosphate processing, 1944-52.

224-U .waste. $\mathrm{LaF}_{3}$ Pu Finishing Plant.

A reduced pressure evaporator in the 200 East Area designed for $30 \%$ solids. Began operations in 1977; continues to operate. Tank $\mathrm{AW}-102$ is the feed tank.

Atmospheric evaporator used for concentrating wastes; operated 1952-56. Tank B-106 was the feed tank.

Reduced pressure evaporator designed for $30 \%$ solids; operated 1973-77. Tank SY-102 was the feed tank from 1977-81.

Atmospheric evaporator used to concentrate wastes; operated from 1952-56 and 1965-76. Tank TX-118 was the feed tank.

The tank design used for the SX Tank Farm. A 3rd generation tank has an operating capacity of $3,790 \mathrm{~kL}$ (1 million gal), a diameter of $22.8 \mathrm{~m}$ (75 ft), a $37.8-\mathrm{cm}(14.8-\mathrm{in}$.) dish bottom, and no knuckle. 
4th Generation Tank

5th Generation Tank

Active Tank

Active Waste

Aging Waste

Airlift Circulator

Annulus

Assumed Leaking

Tank or Assumed Leaker

Assumed Re-leaker

B Plant High-Level Waste
This design was used for the A Tank Farm. These tanks are the same as 3rd generation tanks, except they have flat bottoms.

The design used for the AX Tank Farm. The design is the same as 4th generation tanks with the addition of grid drain slots beneath the steel-liner bottom.

A tank that contains more than $125,000 \mathrm{~kL}(33,000 \mathrm{gal})$ of waste and/or is still involved in waste management operations.

A tank being used, or planned for use, for the storage of liquid in excess of a minimum supernatant liquid heel in connection with production and/or waste processing.

High-level, first-cycle solvent extraction waste from the PUREX Plant neutralized cladding removal waste (see Neutralized Current Acid Waste).

Airlift circulators were installed in tanks used to store aging wastes. The circulators agitate the contents of waste tanks by releasing compressed air near the bottom of the tank through a pipe extending vertically through the wastes. The circulators promote the mixing of liquid wastes and are designed to prevent agglomeration of radionuclides. By maintaining motion within the wastes, circulators minimize superheat build-up, and consequently, minimize bumping.

The space between the primary liner and secondary liner on the double-shell tanks. Drain channels in the insulating and/or supporting concrete carry any leakage to the annulus space where conductivity probes are installed. Alarms from the annunciators are received by Computer Automated Surveillance System (CASS). Continuous air monitoring (CAM) alarms are also located in the annulus. The annulus conductivity probes and radiation detectors are the primary means of leak detection for all double-shell tanks.

The integrity classification of a waste storage tank for which surveillance data indicate a loss of liquid attributed to a breach of tank integrity. In 1984, the criteria designations of suspect leaker, questionable integrity, confirmed leaker, declared leaker, borderline, and dormant were merged into one category now reported as assumed leaker.

A designation for a tank declared an assumed leaker for which the surveillance data indicate a new loss of liquid attributed to a breach of integrity.

Known as B or B Plant HLW. Also identifies waste returned to tanks following strontium recovery. 
Baseline

Bismuth Phosphate Process

Boiling Waste

Bottom Referenced

Tank

Bumping

Cascade

Catch Tanks

Closure

Concentrated

Complexant (CC)

Concentrated

Phosphate Waste

(CP)

Conductivity Probe
A specific program strategy and process steps, selected and approved for carrying out the retrieval technology development part of the Tank Waste Remediation System (TWRS). It will be the basis for establishing the retrieval technology development program scope, cost, and schedule.

First precipitation process (based on $\mathrm{BiPO}_{4}$ ) used at the Hanford Site for separation of plutonium from the irradiated uranium fuels; the process used at $B$ and $T$ Plants from 1944-56. This process was replaced by REDOX and PUREX processes to gain the advantages of separation and recovery of the uranium and plutonium fission products.

Waste containing sufficient radioactive decay heat to self-boil.

Either a dished bottom tank or a flat bottom tank, where the zero point for liquid-level gages is the lowest elevation in the tank.

A sudden, violent evolution of vapor from a boiling liquid, contributed to by superheating.

Eleven of the single-shell tank farms (all except the AX Tank Farm) were equipped with overflow lines between tanks. The tanks were connected in series and placed at descending elevations creating a downhill gradient for liquids to flow from one to another.

Small capacity single-shell tanks associated with diversion boxes and diverter stations. The tanks are designed to receive any transfer line cleanout, spills or leakage from the boxes, or leakage from the adjacent pipe encasement.

Disposition of a hazardous waste treatment, storage, or disposal facility, which has discontinued operation, in accordance with a Washington State-approved closure plan.

Concentrated product from the evaporation of dilute complexed waste, characterized by a high content of organic carbon (see dilute complexed waste).

Waste originating from the decontamination of the $100 \mathrm{~N}$ Area Reactor. Concentration of this waste produces concentrated phosphate waste.

Measures the surface level of conductive liquid (or waste) by detecting electrical conductivity between the probe tip and the liquid/waste surface as it is lowered into contact. 
Confirmed or

Declared Leaker

Crib

Crust

Data Quality

Objectives (DQOs)

Dilute Complexed

Waste (DC)

Dilute Noncomplexed

Waste (DN)

Disposal

Diversion Box

Double-Shell Slurry . (DSS)
The designation of any underground waste storage tank where data are considered sufficient to support a conclusion with $95 \%$ confidence that the tank has leaked.

A linear excavation about $4.6 \mathrm{~m}$ (15 ft) in depth, with a perforated pipe in the bottom. The ditch is backfilled with broken rock or other loose material and then covered by soil and a liquid impermeable membrane; the pipe is then used to distribute liquid wastes along the crib. The radionuclides are absorbed in the soil and the liquid percolates to groundwater.

A hard surface layer that has formed in many waste tanks containing concentrated solutions.

Qualitative and quantitative statements that specify the quality of the data required to support agency decisions during remedial response activities. They are determined based on the end uses of the data to be collected.

Characterized by the presence of organic carbon including organic complexants: ethylenediaminetetracetic acid (EDTA), citric acid, hydroxyethylenediaminetriacetic acid (HEDTA), and iminodiacetate (IDA) being the major complexants used. The main sources of dilute complexed waste in the double-shell tanks are salt well liquid inventory.

Miscellaneous low activity liquid wastes originating form $T$ and $S$ Plants, the 300 and 400 Areas, PUREX Plant (decladding supernatant and miscellaneous wastes), $100 \mathrm{~N}$ Area (sulfate waste), B Plant, salt wells, and Plutonium Finishing Plant (PFP) supernate. Dilute noncomplexed waste is defined as waste with total organic carbon (TOC) $<1 \mathrm{wt} \%$ (or $<10 \mathrm{~g} / \mathrm{L}$ ).

Placement of waste in a manner that ensures isolation from the biosphere for the foreseeable future with no intent of retrieval and requires deliberate action to regain access to the waste.

A below-grade concrete enclosure containing the remotely maintained jumpers and spare nozzles for the routing of waste solution to storage tank farms.

Waste that exceeds the sodium aluminate saturation boundary in the evaporator without exceeding receiver tank composition limits. This waste is a concentrate of double-shell slurry feed (DSSF), but with total organic carbon (TOC) $<10 \mathrm{~g} / \mathrm{L}$. For reporting purposes, DSS is considered a solid. 
Double-Shell Slurry

Feed (DSSF)

Double-Shell Tank (DST)

Drainable Interstitial Liquid

Drywell

Drywell (In-tank)

ENRAF

Evaporator Feed

Ferrocyanide
Waste concentrated just before reaching the sodium aluminate saturation boundary in the evaporator without exceeding receiver tank composition limits. This form is not as concentrated as DSS.

A reinforced concrete underground vessel with two inner steel liners to provide containment of liquid waste; instruments are installed in the annulus to detect leaks from the inner liner.

Liquid that is not held in place by capillary forces, and will, therefore, migrate or move by gravity. Drainable liquid remaining minus supernate. Drainable interstitial liquid is calculated based on the salt cake and sludge columns, using average porosity values or actual data for each tank, when available.

Vertical bore holes with 15.2-cm (6-inch) (internal diameter) carbon steel casings positioned radially outside single-shell tanks. Periodic monitoring is done by gamma radiation or neutron sensors to obtain scan profiles of radiation or moisture in the soil as a function of well depth, which could be indicative of tank leakage. These wells range between 15.2 to $76.2 \mathrm{~m}$ (50 to $250 \mathrm{ft}$ ) in depth, and are monitored between the range of 15.2 to $45.7 \mathrm{~m}$ (50 to $150 \mathrm{ft}$ ). The wells are sealed when not in use. They are called drywells because they do not penetrate to the water table and are, therefore, usually "dry."

A sealed casing within a tank that is attached to a riser. It is used to insert experimental equipment, such as neutron or acoustical probes, to determine the level of drainable interstitial liquor.

The manufacturer of an automatic liquid-level gauge being used to replace the older Food Instrument Corporation gauges in Hanford Site waste tanks. The ENRAF 854 gauge measures surface levels with a polyethylene displacer (weighing about $200 \mathrm{~g}$ ) that is lowered on a wire into a tank. The waste level is determined by a change in the buoyancy of the displacer. The gauges are electrically connected to a computer system for data transmission, analysis, and reporting. Local readings may also be obtained from a liquid crystal display.

Any waste liquid that can be concentrated to form salt cake; for example, low-heat waste, dilute interstitial liquid, aged waste, and other radioactive waste solutions.

An ion composed of iron and cyanide with the chemical formula of $\mathrm{Fe}(\mathrm{CN})_{6}^{-4}$. 
Fission Product Waste (FP)

Food Instrument Corporation (FIC)

Hanford Coordinates

Hanford Federal Facility Agreement and Consent Order (Tri-Party Agreement)

Hanford Waste Vitrification Plant (HWWP)

Heel

Hexone

High-Level Waste

Inactive Tank
Waste produced at the B Plant and Strontium Semiworks in campaigns for cesium and strontium recovery during the 1960's. Cesium was removed from PUREX supernatant and strontium was removed from PUREX sludge; both were removed from acidic waste.

The manufacturer of a automatic liquid-level gauge that measures surface levels with a conductivity probe. At Hanford, the gauges are electrically connected to a computer system for data transmission, analysis, and reporting. Local readings may also be obtained from a dial.

A set of offsets, in feet, from a reference point on the Hanford Site. These are the units used to lay out these facilities.

A legal document (Ecology et al., 1993) that binds the DOE to actions to comply with the Resource Conservation and Recovery Act of 1976 (RCRA); the Comprehensive Environmental Response, Compensation, and Liability Act of 1980; and the State of Washington Hazardous Waste Management Act.

A facility to be constructed for treatment of high-level liquid radioactive waste. Liquids are vitrified or classified in order to reduce the potential for radioactive and hazardous contamination leaking into the environment. This unit will be regulated under RCRA.

The amount of material remaining in a vessel or container after most of the contents have been removed.

Methyl isobutyl ketone, an organic solvent used in the REDOX solvent extraction process.

Highly radioactive waste material that results from the reprocessing of spent nuclear fuel, including liquid waste produced directly in reprocessing, and any solid waste derived from the liquid, which contains a combination of transuranic waste and fission products in concentrations requiring permanent isolation.

Before 1988, a tank that was removed from liquid-processing service, was pumped to less than $125 \mathrm{~kL}(33,000 \mathrm{gal})$ of waste, and was waiting to be, or was in the process of being stabilized and interim isolated. Included all tanks not in active or active-restricted categories. In September 1988, the designation was changed to out-of-service. 
In-Service Tank

Interim Isolated (ISO)

Interim Stabilized

Interstitial Liquid

Intrusion Prevention

Isolation

ITS

Jet Pump

Knuckle
The waste classification of a tank being used, or planned for use, for the storage of liquid (in excess of a minimum supernatant liquid heel) in conjunction with production and/or waste processing. All Hanford Site DSTs are in-service; none of the SSTs are in-service (see also active waste).

The administrative designation reflecting the completion of the physical effort required to minimize the addition of liquids into an inactive storage tank, process vault, sump, catch tank, or diversion box. Pipes into or out of a tank are typically blanked off, and risers and pits covered in a rigid foam (weather coated). In June 1993, the term Interim Isolation was replaced by Intrusion Prevention.

A tank which contains less than $189 \mathrm{~kL}(50,000$ gal) of drainable interstitial liquid and less than $18.9 \mathrm{~kL}(5,000 \mathrm{gal})$ of supernatant liquid. If the tank was jet pumped to achieve interim stabilization, then the jet pump flow must also have been at or below 0.189 $\mathrm{L}$ min $(0.05 \mathrm{gal} / \mathrm{min})$ before interim stabilization criteria are met.

The liquid that fills the interstices or the void volume in the solids waste material in a waste tank. This liquid is estimated to be abouf $50 \%$ of the solids volume. In salt cake, approximately $60 \%$ of the interstitial liquid is drainable and about $40 \%$ is held in place by capillary forces (nondrainable). In the sludge portion of tank farm waste, this liquid is not considered pumpable or drainable but may contain pockets of liquid that cannot be estimated. Interstitial liquid may be evaporator feed or terminal liquid.

The administrative designation reflecting the completion of the physical effort required to minimize the addition of liquids into an inactive storage tank.

The act of sealing a tank against liquid intrusion from credible sources and confining the atmosphere in the tank. Filtered ainways are not sealed; the pressure is balanced to the atmosphere, and in some cases cooling airflow is provided.

The In-Tank Solidification Program, used to reduce waste volume directly by evaporating the wastes from inside certain tanks in the BY Tank Farm.

A modified commercially available jet pump used as a salt well pump. A centrifugal pump recirculates a stream to serve as the motive fluid for the jet located at the bottom of the well.

Point where the side wall and the bottom curved surface of a tank meet. 
Lateral

Leak Detection Pit

Liquid Level (LL)

Liquid Observation Well (LOW)

Low-Level Waste

Metal Waste (MW)

Mitigation

Module

Neutralized Current

Acid Waste (NCAW)
Horizontal drywell under A Tank Farm and certain SX Tank Farm waste storage tanks.

The collection point for leakage from AX Farm single-shell tanks and from all double-shell tanks. The pits are equipped with radiation and liquid-detection instruments.

The waste surface level within an underground storage tank.

Liquid observation wells are used for monitoring the interstitial liquid level (ILL) in single-shell waste storage tanks. The wells are constructed of fiberglass, or tefzel-reinforced epoxy-polyester resin. They extend to within $2.54 \mathrm{~cm}$ (1 in.) of the bottom of the tank steel liner. They are sealed at their bottom ends and have a nominal outside diameter of $8.89 \mathrm{~cm}$ (3.5 in.). Three probes are used to monitor changes in the interstitial liquid level: acoustic, gamma, and neutron. The LOW can indicate intrusions or leakage by increases or decreases in the interstitial liquid level. There are 58 liquid observation wells (57 are in operation) installed in SSTs that contain or are capable of containing greater than $189 \mathrm{~kL}(50,000$ gal) of drainable interstitial liquid, and in two DSTs only. The liquid observation wells are for surveillance purposes only.

Waste that contains radioactivity and is not classified as high-level waste, transuranic waste, spent nuclear fuel, or by-product material. Test specimens of fissionable material irradiated for research and development only, and not for the production of power or plutonium, may be classified as low-level waste, provided the concentration of transuranics is less than $100 \mathrm{nCi} / \mathrm{g}$.

Waste from the bismuth phosphate process containing all the uranium, about $90 \%$ of the original fission product activity, and about $1 \%$ of the product. This waste was brought to just the neutral point with $50 \%$ caustic and then treated with an excess of carbonate. This procedure yielded almost completely soluble waste to a minimum total volume. The exact composition of the carbonate compounds was not known, but was assumed to be uranium phosphate carbonate mixture (see also $1 \mathrm{C}$ and $2 \mathrm{C}$ ).

Reduction of the severity of a tank safety issue.

A tank grouping made according to similar waste characteristics.

Primary high-level waste stream from the PUREX process. 
Neutralized PUREX Acid Waste

Neutron Probe

Noncomplexed (NCPLX)

Normal Paraffinic Hydrocarbon (NPH)

Organic Wash Waste (owm)

Out-of-Service Tank

Partial Neutralization Feed (PNF)

Partially Interim Isolated (PI)

Pretreatment

Primary Stabilization

Probe

Pumpable Liquid

Remaining
High-level waste was originally neutralized at the PUREX Plant and sent to the 241-A Tank Farm. As fission product recovery began, a portion of the waste was treated for strontium recovery and then neutralized. As of 1967, all high-level waste left PUREX as an acid solution for treatment at B Plant.

Probe equipped with a neutron source and detector. They are used in drywell monitoring to determine the moisture content of the soil as one means for detecting leaks in underground waste storage tanks or pipelines.

General waste term applied to all Hanford Site liquids not identified as complexed (i.e., wastes not containing organics).

Diluent used in uranium recovery and PUREX processes; is close to dodecane, $\mathrm{C}_{12} \mathrm{H}_{26}$.

The solvent used in the PUREX process was treated before reuse by washing with potassium permanganate and sodium carbonate, followed by dilute nitric acid. Often assumed to be $1: 1$ with PUREX high-level waste (HLW).

A tank that does not meet the definition of an in-service tank. Before September 1988, these tanks. were defined as inactive. All single-shell tanks are out of service.

Waste stream with nitric acid added at an evaporator in an attempt to produce more salt cake during volume reduction.

The administrative designation reflecting the completion. of the physical effort required for interim isolation, except for the isolation of risers and piping required for jet pumping or for other methods of stabilization:

Chemical treatment process or a series of processes used to prepare waste for immobilization.

The condition of an inactive waste storage tank after all supernatant liquid, " other than isolated surface pockets, has been removed. Isolated surface pockets of liquid are those not pumpable by conventional techniques.

An instrument package designed to be inserted in dry wells, tank risers, or other access ports to measure conductivity, radiation, moisture, or temperature.

Drainable liquid remaining less nondrainable heel volume. 
PUREX Plant

PUREX Acidified

sludge (PAS)

PUREX Decladding

Questionable Integrity

REDOX

Relative Percent

Difference

Relative Standard

Deviation

Remediation

Resolution

Resource

Conservation and

Recovery Act (RCRA)

Riser

Salt Cake

Salt Well
Plutonium-Uranium Extraction Plant (also called A Plant). Operated from 1952 to 1972, and then was placed in standby. Ran again from November 1983 to 1991 , and is now shut down.

Sludge that has been sluiced from waste tanks and acidified to $0.1 \mathrm{M} \mathrm{HNO}_{3}$ as part of $\mathrm{Cs} / \mathrm{Sr}$ recovery in the AR Vault.

PUREX neutralized cladding removal waste (NCRW) is the solids portion of the PUREX plant neutralized cladding removal waste stream; the waste is received in tank farms as a slurry. Neutralized cladding removal waste solids are classified as transuranic (TRU) waste.

Any waste storage tank that has a small decrease in liquid level or a radiation increase in an associated drywell for which the data are insufficient to support a conclusion with $95 \%$ confidence that the tank is sound.

Reduction-oxidation process. Plant where redox process ran, approximately 1956-67.

$R P D=A 1-B 1 /(A+B / 2) * 100$

RSD $=(\text { Standard Deviation/Mean })^{\star} 100$

Actions taken to safely store, maintain, treat, and dispose of tank wastes.

Elimination of a tank safety issue by physical, chemical, analytical, and/or administrative methods.

A federal law enacted in 1976 that regulates the generation, transportation, treatment, storage, and disposal of hazardous wastes.

The upright pipe projection from a tank; any vertical pipe.

Results from crystallization and precipitation after concentration of liquid waste, usually by evaporator. Crystallized nitrate and other salts deposited in waste tanks. If salt cake is layered over sludge, it is only possible to measure total solids volume. In-tank photographs may be used to estimate the salt cake volume.

A screened well casing inserted into waste tank solids, extending to near the bottom of the tank. The well may contain a jet pump to remove interstitial liquid that drains into the well from surrounding solids. 
Salt-Well Pump

Scavenged Waste

Self-Concentrating Waste

Single-Shell Tank (SST)

Sluiced or Sluicing

Sludge

Slurry

Sound

Stabilization

Strontium Semiworks

Supernatant Liquid or Supernate

Surface Levels

T Plant
A low-capacity pump used to remove interstitial liquid from salt wells.

Waste which has been treated with ferrocyanide to remove cesium from the supernatant by precipitating it into a sludge.

High-level liquid radioactive waste with constituent radionuclides contributing sufficient decay heat to cause the solution to boil and/or self-concentrate.

An underground waste storage tank with a single metal liner encased in a reinforced concrete shell. There are 149 SSTs at the Hanford Site, ranging in capacities from $208 \mathrm{~kL}(55,000 \mathrm{gal})$ to $3,790 \mathrm{~kL}$ (1 million gal).

To dissolve or suspend in solution by action of a high pressure waste stream to allow the pumping of solids.

Solids formed during sodium hydroxide additions to waste. Sludge usually was in the form of suspended solids when the waste was originally received in the tank from the waste generator. In-tank photographs may be used to estimate the volume.

Insoluble material suspended in water or an aqueous solution.

The integrity classification of a waste storage tank for which surveillance data indicate no loss of liquid from a breach of integrity.

The removal, or immobilization, as completely as possible, of the liquid contained in a radioactive waste storage tank by salt well pumping, adding diatomaceous earth, etc.

Also called C Plant or Hot Semiworks, was used as a pilot plant for both the REDOX and PUREX processes, and was then reconfigured for strontium recovery.

Liquid above the solids in waste storage tanks.

The surface level measurements in all waste storage tanks are monitored by manual or automatic conductivity probes, and recorded and transmitted for input to the Computer Automated Surveillance System (CASS) and/or the Tank Monitoring and Control System (TMACS).

Decontamination plant for various equipment. Originally built for the bismuth phosphate process, but only used for decontamination since 1956. 
Tank Farm

Terminal Liquor (TL)

Thermocouple

Total Waste

Transuranic (TRU)

U Plant

UR Waste
Area containing a number of storage tanks (e.g., the chemical tank farm, which stores chemicals used in a plant, or the underground waste tank farm, which stores radioactive waste).

The liquid product from the evaporation-crystallization process which, upon further concentration, would form an unacceptable solid for storage in single-shell tanks. Terminal liquor is characterized by a caustic concentration of approximately $5.5 \mathrm{M}$ (the caustic molarity will be lower if the aluminum salt saturation is reached first.

A thermoelectric device used to measure temperature. An assembly with multiple thermocouples is called a tree. In doubleshell tanks there may be one or more thermocouple trees in risers in the primary tank. In addition, in DSTs only, there are thermocouple elements installed in the insulating concrete, the lower primary tank knuckle, the secondary tank concrete foundation, and in the outer structural concrete. These monitor temperature gradients within the concrete walls, bottom of the tank, and the dome space. In single-shell tanks, there may be one or more thermocouple trees installed directly in a tank, although some single-shell tanks do not have any trees installed. A single thermocouple may be installed in a riser, or lowered down an existing riser or liquid observation well. There are also four thermocouple laterals beneath Tank A-105, in which temperature readings are taken in 34 thermocouples.

As used in this document and Tank Characterization Reports, the solids volume plus supernatant liquid in a waste storage tank.

An element with an atomic number $>92$; also, a regulatory designation for waste material having $>10 \mathrm{nCi} / \mathrm{g}$.

Uranium recovery plant operated from March 1952 to January 1958, then termed $\mathrm{UO}_{3}$ plant during operation until September 1972. Restarted in March 1984 and is now shut down.

Uranium recovery operation in U Plant operated from 1952-57. Created TBP waste and ferrocyanide scavenging wastes. 
Watch List

A list of tanks that may pose an offsite release hazard from the following potential sources: trap and accumulate hydrogen gas, contain organic salts, contain ferrocyanide $\left(\mathrm{Fe}(\mathrm{CN})_{6}^{-4}\right)$, or have a high-heat generation rate. Special restrictions have been placed on these tanks by "Safety Measures for Waste Tanks at Hanford Nuclear Reservation," Section 3137 of the National Defense Authorization Act for Fiscal Year 1991, Public Law No. 101-501 (also known as the Wyden Amendment). The bill provided specific direction to the Hanford Site operating contractor for monitoring tanks with certain potential hazards. 